\title{
PRELIMINARY CHARACTERIZATION \\ OF THE F-AREA \\ RAILRoAd CROSSTIE PILE \\ AT THE \\ SAVANNAH RIVER SITE
}

\author{
PREPARED FOR \\ WESTINGHOUSE SAVANNAH RIVER COMPANY \\ SAVANNAH RIVER SITE \\ AIKEN, SOUTH CAROLINA
}

\author{
PREPARED BY \\ SIRRINE ENVIRONMENTAL CONSULTANTS \\ AIKEN, SOUTH CAROLINA \\ SIRRINE PROJECT NUMBER \\ G1106 \\ OCTOBER 1991
}

DISTRIBUTION OF THIS DOCUMENT IB UNLIMITED

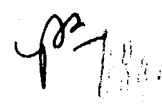


TABLE OF CONTENTS

EXECUTIVE SUMMARY

1.0 PRELIMINARY UNIT EVALUATION

1.1 Introduction 1

1.2 Existing Literature and Information 1

1.3 Waste Characteristics 2

2.0 ENVIRONMENTAL SETTING 3

2.1 Geologic Setting 6

$\begin{array}{ll}2.2 \text { Hydrostratigraphy } & 10\end{array}$

3.0 SUMMARYREPORTOE FIELD OPERATIONS 22

3.1 Field Activities Summary 22

3.2 Sample Collection, Handling, Packaging and Shipping 31

3.3 Quality Assurance/Quality Control 34

3.4 Project Field Files $\quad 35$

4.0 UNIT SCREENING RESULTS 35

4.1 Data Development 35

4.2 Findings 36

4.2.1 Metals in Soil Samples 36

4.2.2 Organic Analytes in Soil Samples $\quad 36$

4.2.3 Other Analytes in Soil Samples $\quad 37$

4.2.4 Metals in TCLP Samples

4.2.5 Radionuclides in Soil Samples 37

4.2.6 Analytes Detected in Groundwater Samples 38

REFERENCES

REF-1

\section{DISCLAIMER}

This report was prepared as an account of work sponsored by an agency of the United States Government. Neither the United States Government nor any agency thereof, nor any of their employees, makes any warranty, express or implied, or assumes any legal liability or responsibility for the accuracy, completeness, or usefulness of any information, apparatus, product, or process disclosed, or represents that its use would not infringe privately owned rights. Reference herein to any specific commercial product, process, or service by trade name, trademark, manufacturer, or otherwise does not necessarily constitute or imply its endorsement, recommendation, or favoring by the United States Government or any agency thereof. The views and opinions of authors expressed herein do not necessarily state or reflect those of the United States Government or any agency thereof. 


\section{EIGURES}

Number

Title

Page

2-1 Site Location and Physiographic Setting $\quad . \quad 4$

2-2 Physical Setting of the F-Area Railroad Crosstie Pile 5

2-3 General Stratigraphic Column for Coastal Plain Sediments 7

2-4 Stratigraphic Section Through SRS $\quad 8$

2-5 General Hydrogeologic Units at SRS 11

2-6 Water Table Contour Map and Flow Direction for the F-Area 12

2-7 Layout Map for Well Clusters P-28 and FC-2 14

2-8 Hydraulic Heads in Groundwater at Well Cluster P-28/FC-2 15

2-9 Regional Potentiometric Surface Map, Aquifer Unit IIA 17

2-10 Areas of Upward and Downward Vertical Hydraulic 18

2-11 Relationship Between Physiography and Hydrostratigraphy Near F- and H-Areas 


\section{IABLES}

Number

Iitle

Page

3-1 F-Area Railroad Crosstie Site Chronological Field Activities Summary

3-2 Soil Sample Matrix, FRT-1HP 26

$\begin{array}{lll}3-3 & \text { Soil Sample Matrix, FRT-5HP }\end{array}$

3-4 Soil Sample Matrix, FRT-4HP 28

$\begin{array}{lll}3-5 & \text { Soil Sample Matrix, FRT-3HP } & 29\end{array}$

3-6 Water Sample Matrix 30

3-7 Soil Sampling Intervals and Analyses 32

3-8 Groundwater Analyses 33

4-1 Metals Detected in F-Area Tie yard Soil Samples 39

4-2 Organic Analytes Detected in F-Area Tie Yard Soil Samples 40

4-3 Cther Analytes Detected in F-Area Tie Yard Soil Samples 41

4-4 Metals Detected in F-Area Tie Yard TCLP Samples 42

4-5 Radionuclides Detected in F-Area Tie Yard Soil Samples 43

4-6 Soil Sample Radionuclide Summary, F-Area Railroad 44

4-7 Groundwater Analyses $\quad 45$

4-8 Background Soil Concentrations, Blanton Soil Series 46

4-9 Background Soil Concentrations, Fuquay Soil Series 47

4-10 Background Soil Concentraionts, Udorthent Soils 48

4-11 Drinking Water Standards $\quad 49$ 


\section{ARPENDICES}

Appendix A Analytes Not Found at the F-Area Railroad Crosstie Pile

Appendix B Groundwater Monitoring Data, FBP Wells

Appendix C Field Notes and Geologic Logs

Note: Laboratory Analytical Results are summarized in the body of the report and are available from Tom Gaughan, WSRC-ER; (404) 821-8016 


\section{EXECUTIVE SUMMARY}

Historical information about the F-Area Railroad Crosstie Pile is limited. The unit is believed to have been a borrow area for earth fill that began receiving railroad crossties during the 1960s. The number of crossties at the unit began to increase significantly in 1984 when major repair of the SRS rail system was initiated. An estimated 100,000 used railroad crossties have accumulated at the unit since 1984.

In an effort to determine the impact of the railroad crossties on the environment, a total of 28 soil samples were collected from four test borings in March of 1991. Sample depths ranged from ground surface to 21.5 feet. Three of the borings were extended to the water table and groundwater samples were collected, one in an upgradient "background" area, and two downgradient from the unit.

Fow analytes were reported above detection limits. Test results are summarized in Section 4.0 and analytes not detected are summarized in Appendix $A$ to this report. In three soil samples collected from depths beiween 10 and 21.5 feet, copper occurred at levels slightly above background. These copper values were detected in the sidegradient test boring and in the two downgradient test borings. Three organic analytes, acetone, pyridine, and Toluene, were reported above detection limits but well below drinking water standards (DWS) in all test borings, including the upgradient boring.

Radionuclide activities were reported above background in both soil and water samples from all test borings (Tables 4-5 and 4-7). There do not appear to be any statistically significant trends in radionuclide activities with depth, or between upgradient or downgradient borings.

The organic analytes tetrachloroethylene and trichloroethylene were detected above DWS in the groundwater in one of the downgradient borings, and well above DWS in the upgradient boring along with carbon tetrachloride and trichloroflouromethane. No organic analytes were detected in groundwater in the second downgradient test boring.

The analytes detected in the test borings downgradient from the unit cannot be attributed to the railroad crosstie pile as they are not significantly different than the values reported for the upgradient, background test boring. 


\section{PRELIMINARY CHARACTERIZATION OF THE F-AREA RAILROAD CROSSTIE PILE AT THE SAVANNAH RIVER SITE}

\subsection{PRELIMINARY UNIT EVALUATION}

1.1 Introduction

A reviow of existing literature and information about the F-Area Railroad Crosstie Pile indicates that the history of the site is not fully documented. However, experienced personnel believe the site began receiving railroad crossties during the 1960s'. The volume of used crossties stored at the site began to increase significantly in 1984 when the first major repair of the SRS rail system, and the construction of L-Lake were initiated1. Since 1984, the FArea Railroad Crosstie Pile has accumulated an estimated 100,000 railroad crossties from repair and replacement of onsite railroad tracks. In addition, the pile includes an unknown number of creosote treated utility poles from power line and telephone system repairs'.

\subsection{Existing Literature and Information}

The following information specifically pertaining to the F-Area Railroad Crosstie Pile was reviewed:

Bignell, D.T., Bailroad Crosstie Disposal Alternatives, Januany. 1991. Letter to Mr. Donald Gross, Jr.

AAR, 1988, Ioxicity Characteristic Leaching Procedure Testing of Railroad Crossties (R-708), Association of American Railroads, Chicago, IL;

Olson, C., Wells Monitored by the Health Protection Department. HPR-88-001

Additional documents pertaining to general SRS waste management activities were also reviewed as necessary and are included in the list of references. 


\subsection{Waste Characteristics}

The F-Area Railroad Crosstie Pile was used for the disposal or staging primarily of creosote-treated railroad crossties. In addition, the site has also received some creosote-treated utility poles, guardrail posts, fence posts, and other miscellaneous lumber ${ }^{1}$. Creosote, a petroleum based wood preservative and insect repellent, may contain up to 200 various molecular weight organic compounds. Chemical components of, and additives to, creosote may include cresols, phenolic compounds such as pentachlorophenol, heterocyclic compounds, and various three-to-six carbon-chain volatile organic acids. Most of these compounds form the soluble, and hence leachable, phase of creosote. The soluble constituents/components of creosote are prone to natural degradation, which may yield many intermediate and end products. Degradation components of creosote include: phenol (primary intermediate compounds), various methylated phenols, methane, and carbon dioxide.

Waste railroad ties obviously meet the definition of a solid waste, but not necessarily a hazardous waste. A solid waste is classifled as a hazardous waste if it exhibits the characteristics of ignitability, corrosivity, reactivity, or toxicity as defined in 40 CFR 261, Subpart C. Creosote contains cresol and pentachlorophenol compounds which fall into the category of regulated hazardous constituents. Representative samples were obtained from SRS ties of $1953,1961,1975,1984$, and 1990 vintage and were analyzed. The results from those TCLP tests, and the tests from eleven previous SRS samples (also past technical reports from the Association of American Railroads ${ }^{2}$ ) indicate that the ties are non-hazardous'.

All used ties at the F-Area storage site were removed from a nonradioactive (clean) area, i.e., not from within a Radiologically Controlled Area (RCA). However, the possibility does exist that some ties may have been radioactively contaminated due to the possibility of a railroad car being contaminated1. This potential for radiological contamination necessitates that radiological requirements be considered and evaluated when analyzing the alternatives for crosstie disposal (particularly those options involving transporting the ties offsite). While beta and gamma radiation can be readily monitored with existing instruments, alpha radiation is easily shielded making monitoring very difficult. 
An alpha emitting contamination that is not on the surface of the wood may be difficult to impossible to detect with present SRS scanning instruments'. The ties must be completely surveyed, internally and externally, for alpha and betagamma contamination before offsite shipments are allowed to any contractor, other than a contractor permitted by Federal and State regulatory agencies to dispose of radioactive wastes.

\subsection{ENVIRONMENTAL SETTING}

The Savannah River Site is located on the Upper Coastal Plain about 20 miles southeast of the Fall Line in west-central South Carolina. F-Area is located in the central portion of the Site, as shown on Figure 2-1. The F-Area Railroad Crosstie Pile is situated on a ridge in an area of varied vegetation which includes mature broad leaf and pine trees with sparse ground cover. The undergrowth has been cleared from the surrounding area by the U.S. Forest Service to provide a fire break. The crossties are positioned in an area that has been leveled by excavating into the west side of a low hill, previously a soil borrow area. A very steep erosional gully approximately 70 feet deep is immediately to the west of the site. The gully drains toward Upper Three Runs about 1300 teet to the northwest. The closed F-Area Burning/Rubble Pits and Scrap Lumber Pile were located in a cleared area about 300 feet to the south of the railroad crosstie pile. The physical setting of the Railroad Crosstie Pile is depicted on Figure 2-2.

The predominant soil series in the vicinity of the F-Area Railroad Crosstie pile are classified within the Blanton and Fuquay Series and as Udorthents ${ }^{3}$. Udorthent is a generic term for sites where the natural soil horizons have been disrupted, usually by construction activities. 


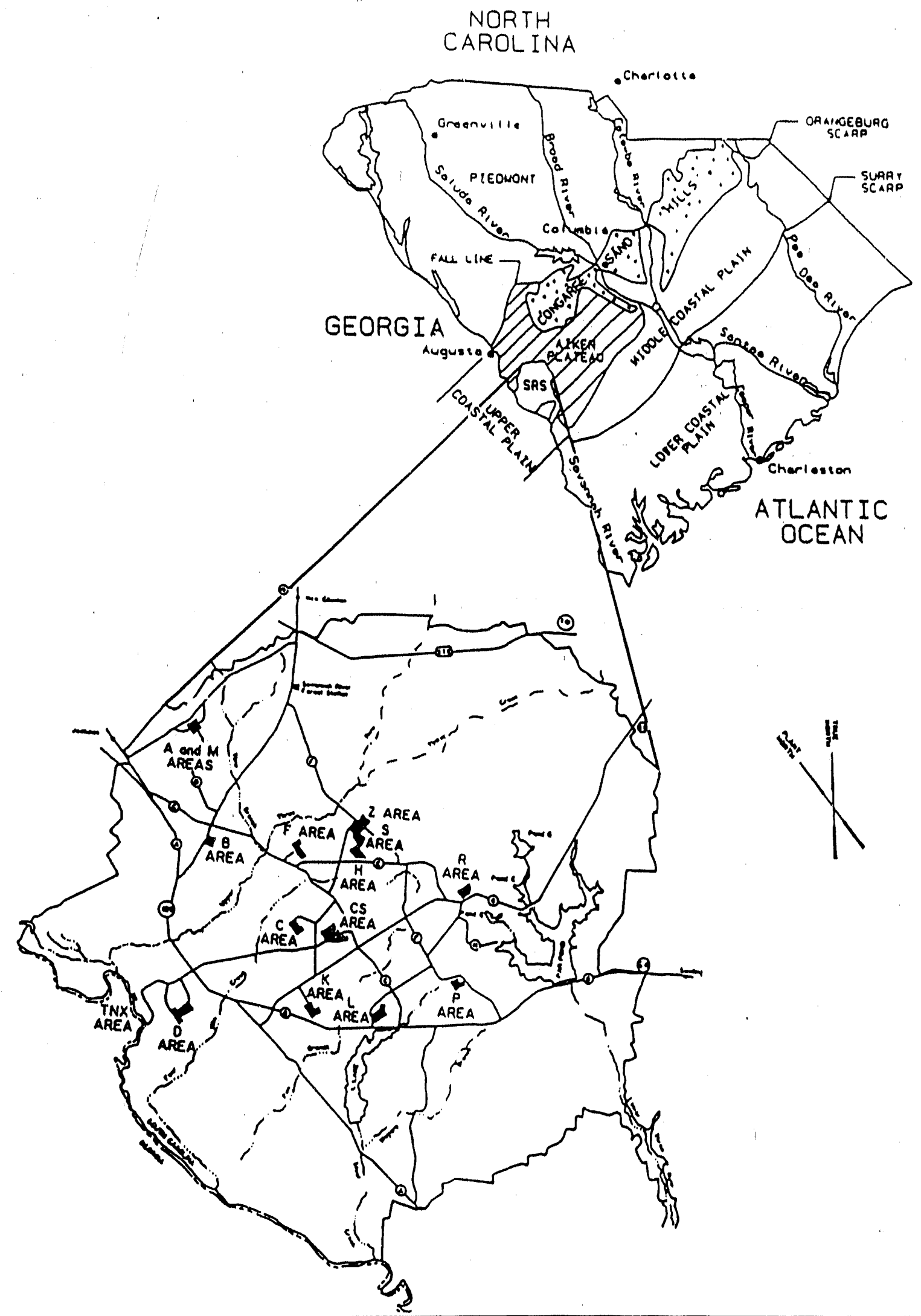

FIGURE 2-1 SITE LOCATION AND PHYSIOGRAPHIC SETTING 

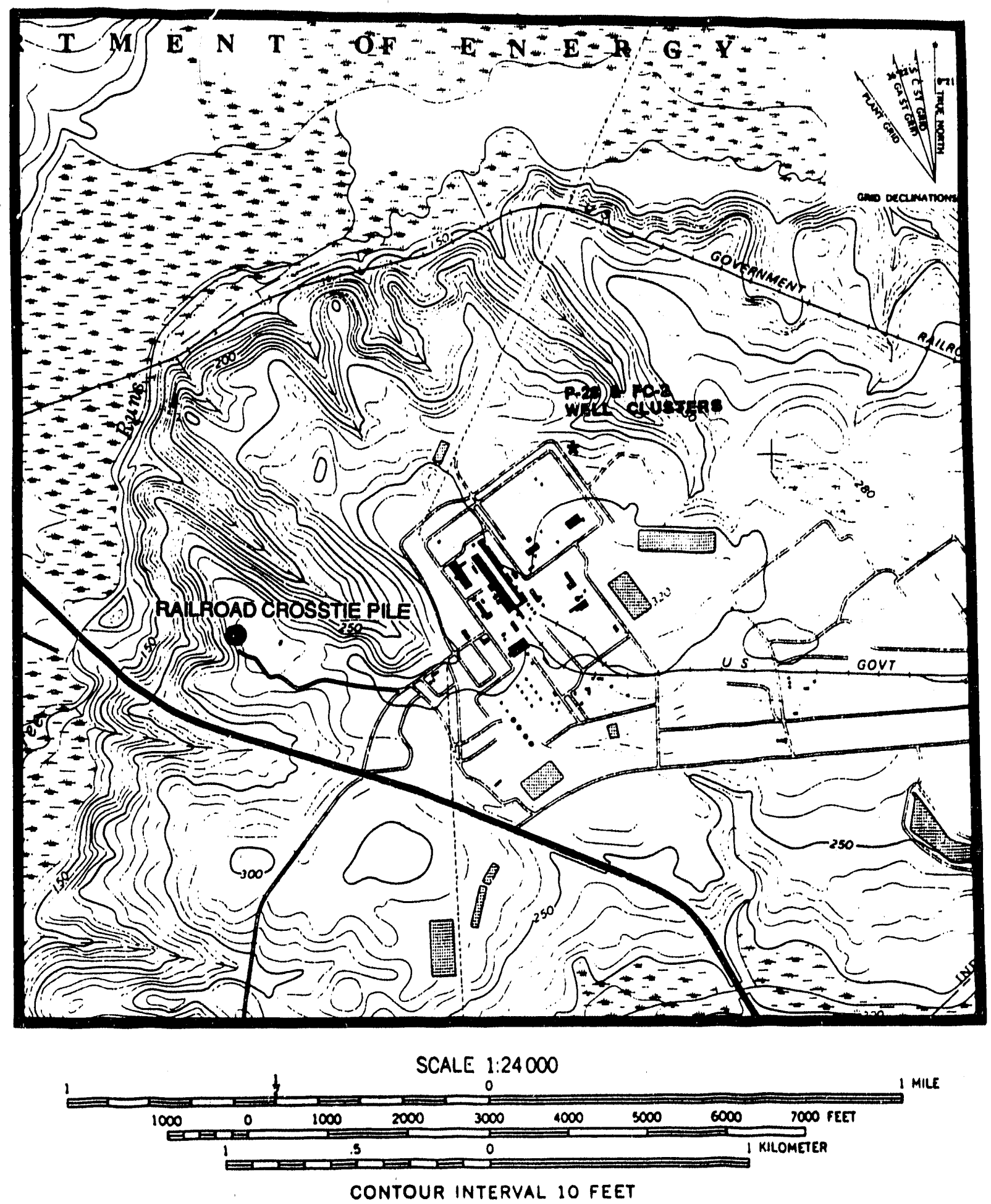

Adapted from USGS 7.5 Minute Quadrangle: New Ellenton SW, Rev. 1981

FIGURE 2-2 PHYSICAL SETTING OF THE F-AREA RAILROAD CROSSTIE PILE 


\subsection{Geologic Setting}

The subsurface geology that underlies the SRS includes crystalline basement rocks and Triassic sedimentary rocks which are overlain by Cretaceous and younger Coastal Plains sediments. At SRS the depth to bedrock ranges from approximately 750 feet at the northern boundary to more than 1400 feet to the south4. The crystalline basement rock is composed of chlorite-hornblende schist, hornblende gneiss, and lesser amounts of other types of crystalline rock. The Triassic sedimentary bedrock occurs in the southern portion of the Savannah River Site and consists of poorly sorted conglomerate, sandstone and mud-rock. These are generally dark red in color.

The stratigraphic units of the Coastal Plain, from oldest to youngest, are the Cape Fear Formation, the Lumbee Group, the Black Mingo Group, the Orangeburg Group, the Barnwell Group, and the Upland Unit, as depicted on Figure 2-35. A stratigraphic section across SRS is depicted on Figure 2-46. The lowermost of these units, the Cape Fear Formation, is composed of Lower Cretaceous, poorly sorted, sitty to clayey quartz sands and interbedded clays which range in thickness from 30 feet at the northwest site boundary to more than 180 feet at the southeast site boundary.

The Lumbee Group (Upper Cretaceous) is subdivided into the Middendonf, the Black Creek, and the Pee Dee Formations. The lower contact between the Middendorf and the Cape Fear is a sharp erosional unconformity. In general, the Lumbee Group is composed of variably colored quartz sands and clays which range in thickness of 380 to more than 700 feet from the northwestern site boundary to the southeastern site boundary respectively.

The Black Mingo Group consists of the Rhems Formation (Early Paleocene), which includes the Ellenton Member, and the Williamsburg Formation (Late Paleocene). The Ellenton consists mostly of gray, poorly sorted, micaceous, lignitic, silty and clayey quartz sand interbedded with gray clays. The Ellenton is approximately 40 feet thick at the northwestern boundary of the SRS and thickens to approximately 100 feet at the southeastern boundary. The Williamsburg sediments are composed of silty quartz sand interbedded with 


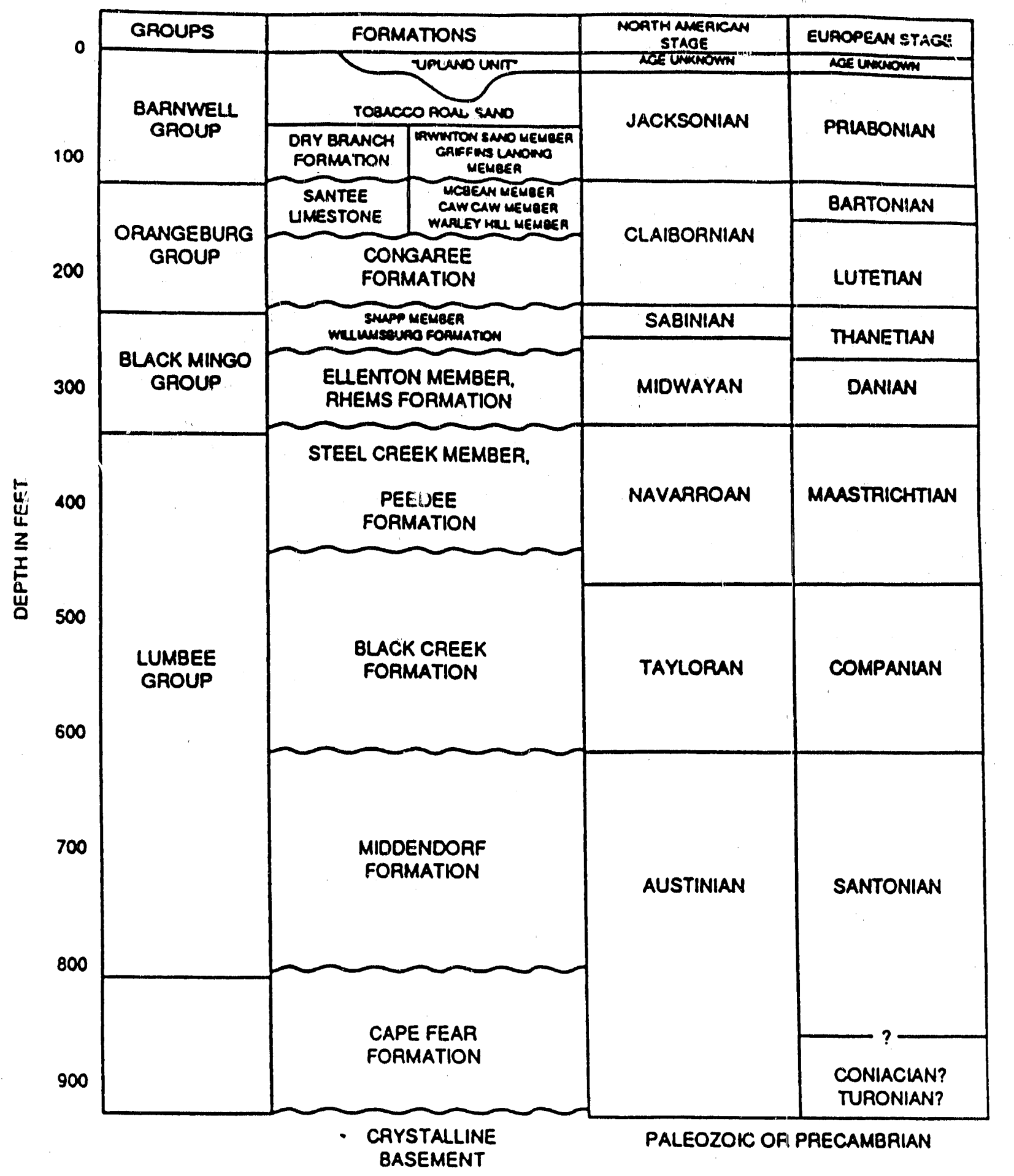

Modified from Price, et al. 5

FIGURE 2-3 GENERAL STRATIGRAPHIC COLUMN FOR COASTAL PLAIN SEDIMENTS 


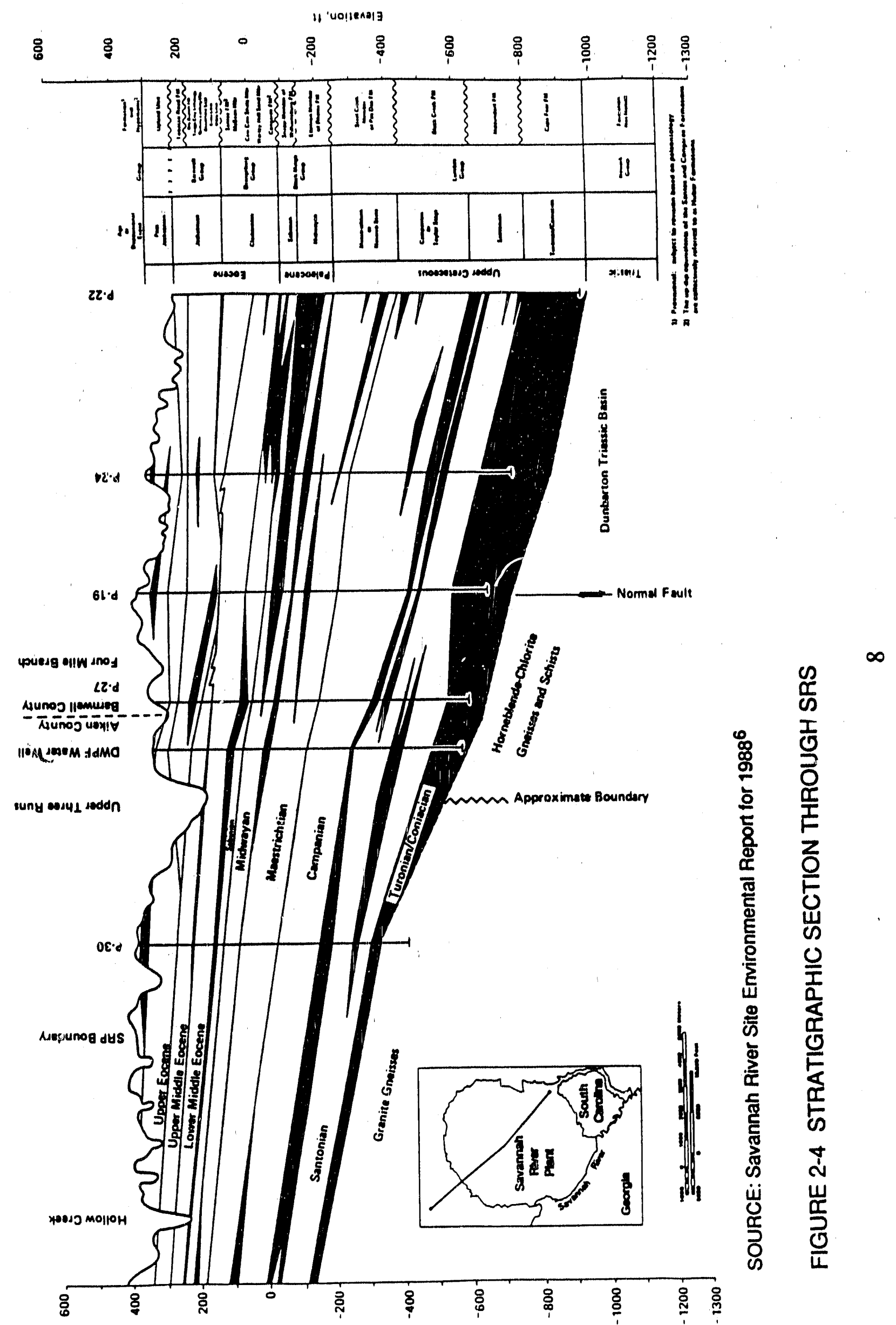


clays. The Williamsburg thickens from 30 feet at the northwestern boundary of SRS to 50 feet at the southeastern boundary.

The Orangeburg Group is subdivided into the lower Middle Eocene Congaree Formation and the Upper Middle Eocene Santee Limestone Formation. The Congairee is composed of well sorted, fine-to-coarse quartz sands with thin clay laminae throughout. The Congaree is approximately 60 feet thick at the northiwestern boundary of the SRS and thickens to approximately 85 feet near the southeastern boundary. The Santee Limestone Formation is subdivided into the Warley Hill, the Caw Caw, and the McBean Members, and vari6;'s in lithology from carbonates and calcareous quartz sands to quartz and glauconitic sands and clays. The Warley Hill and the Caw Caw Members make up what has been referred to in many SRS reports as the "Green Clay". This unit is composed of glauconitic, silty and clayey quartz sands. The thickness of the formation ranges from 40 feet to more than 80 feet from the northwestern boundary of the SRS to the southeastern boundary.

The Barnwell Group (Late Eocene) is subdivided into the Clinchfield, the Dny Branch, and the Tobacco Road Formations. The Clinchfield is present at some locations at the SRS but it is not continuous enough to be correlated across the Site. The Dry Branch is composed of silty and clayey quartz sand with interbedded clays. The Dry Branch Formation ranges in thickness from 50 feet to 80 feet from the northwestern boundary of the SRS to the southeastern boundary. The Tobacco Road Formation consists of massive moderately to poorly sorted quartz sand with minor clay laminae. The Tobacco Road outcrops at the surface at many locations at the SRS and ranges in thickness from 20 to 30 feet.

The Upland Unit (previously mapped at SRS as the Hawthorn Formation) is composed of clays and poorly sorted, ciayey, silty, pebbly, and cobbly quartz sands with extreme lateral and vertical variation. 7 These sediments represent fluvial channel deposits. The Upland Unit is about 15 feet thick in this area. 


\subsection{Hydrostratigraphy}

A multilayer hydrologic system exists in the Coastal Plain sediments at the Savannah River site within which confining units are interspersed with more transmissive units. Aadland and Bledsoe 4 have proposed a new hydrostratigraphic nomenclature system (Figure 2-5) which will be used in this report.

Aquifer Unit IIB is the shallow water table aquifer at the SRS, composed of sediments from the Tobacco Road, the Dry Branch, and the McBean Formations. The sediments of Aquifer Unit IIB exhibit a wide variation in lithologic composition both vertically and laterally. The position of the water table results more from unconfined aquifer conditions than from stratigraphic control.

Aadland and Bledsoe 4 report a series of discontinuous thin clay lenses (informally referred to as the "Tan Clay") within the Dry Branch Formation in Fand $\mathrm{H}$-Areas. This serves as a locally significant aquitard, designated Confining Zone IIB1-\|B2. This confining zone divides Aquifer Unit IIB into a lower aquifer zone,IIB1, and an upper aquifer zone, IIB2.

Based on water level data from wells and test borings near the F-Area Railroad Crosstie Pile, the depth to water in the area is about 100 feet below land surface. The groundwater flow direction of Aquifer Unit IIB (based on average measurements recorded for 1988) in the F-Area was shown to be to the westnorthwest. Contours of the water table aquifer are depicted on Figure 2-6. Values reported for hydraulic conductivity of Aquifer Unit IIB range from $1 \times 10^{-3}$ $\mathrm{cm} / \mathrm{sec}$ to $1 \times 10^{-6} \mathrm{~cm} / \mathrm{sec}$ depending on the type of geologic material across which the wells are screened.

Aquifer Unit IIB is separated from Aquifer Unit IIA (Congaree) by Confining Unit IIA-IIB. This confining unit (described in previous SRS reports as the Green Clay or Caw Caw Clay Member) was identified at a depth of 152 feet below land surface in Well P-28 TA. 


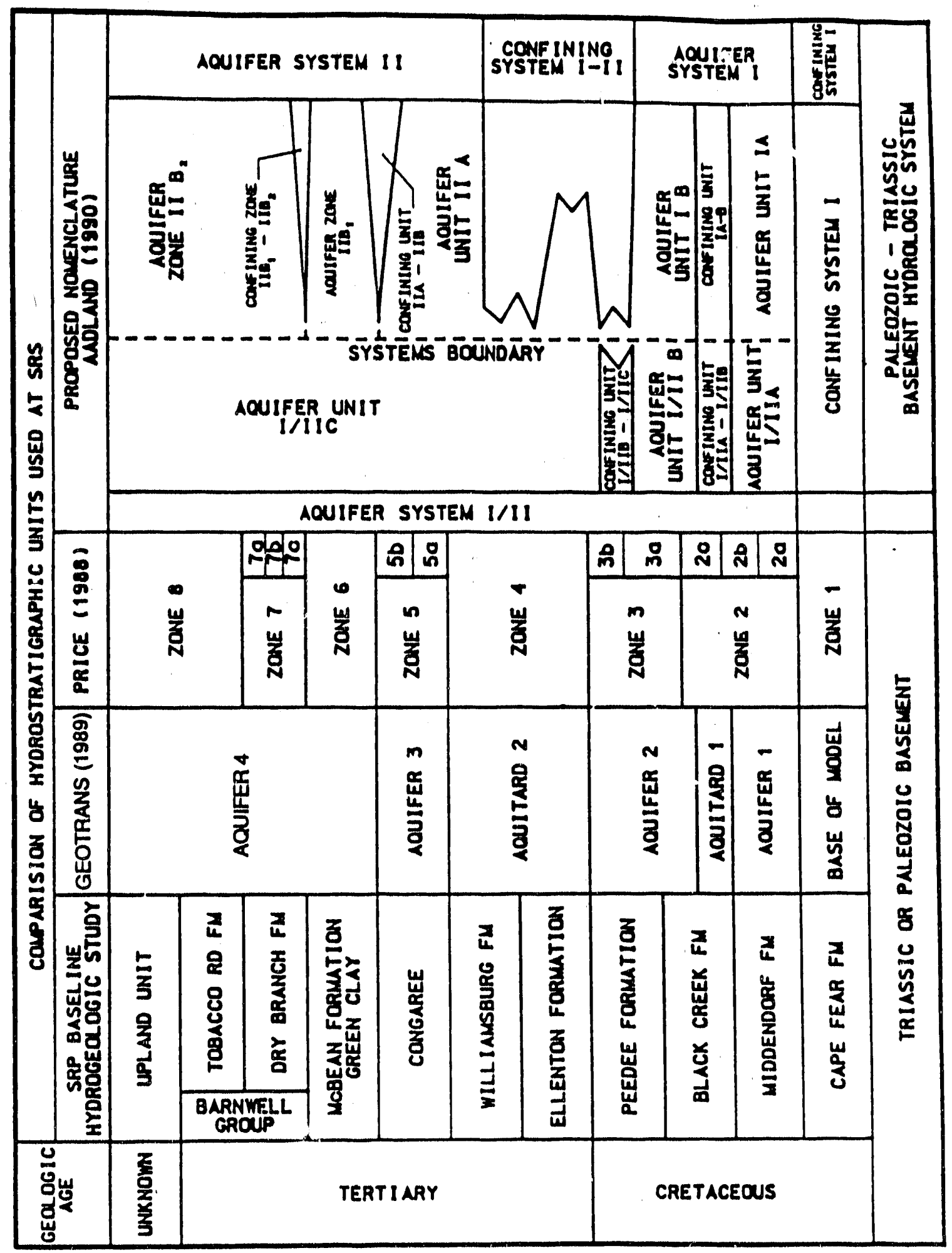

FIGURE 2-5 GENERAL HYDROGEOLOGIC UNITS AT SRS 


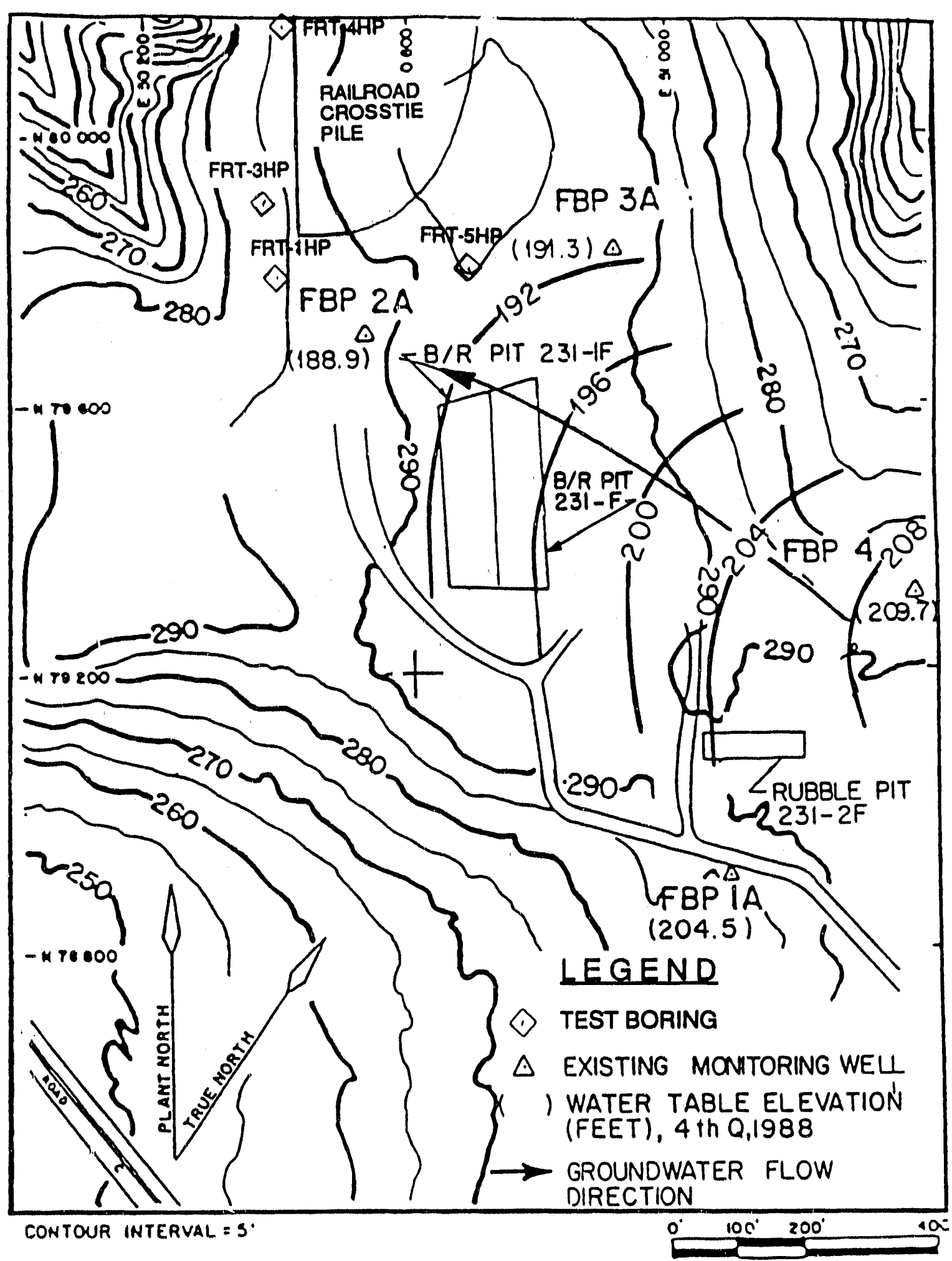

Modified from HPR-88-001, $1988^{8}$

FIGURE 2-6 WATER TABLE CONTOUR MAP AND FLOW DIRECTION FOR THE F-AREA RAILROAD CROSSTIE PILE VICINITY, (4th Quarter, 1987) 
The general hydrologic conditions in the vicinity of the F-Area can be observed from the P-28/FC-2 well cluster, located 4700 feet east of the rallroad crosstie pile (see Figure 2-2). In all, a total of 12 wells are found at this location, approximately 110 feet east of the F-Area perimeter. Ground surface elevations range from 280 feet, to 286 feet. A detailed location map and individual well layout for both clusters can be found on Figure 2-7.

Profiles for the P-28 and FC-2 clusters are presented on Figure 2-8 along with the approximate locations of the major clay and clayey zones. All wells within the FC-2 cluster are completed in the Tertiary age formations of Aquifer System II above confining System I-ll (Ellenton Formation). Wells in this cluster range in depth from approximately 80 feet for the water table well (FC-2F) to approximately 235 feet for the Aquifer Unit IIA well (FC-2A) which is completed in the lower Congaree. The six wells installed in the P-28 cluster are all completeo in the Cretaceous age formations of Aquifer System I below Confining System 1-ll. Wells in this cluster range in depth from approximately 240 feet for the uppermost well screened in the first permeable sand unit below the Ellenton and upper Cretaceous clays (P-28A) to approximately 780 feet for the deepest well at the site, P-28TA, which is screened in Aquifer System I in the lower Middendorf formation.

The water table is found at a depth of about 70 feet below the ground surface (approximate elevation 217 feet) in well FC-2F. Below the water table, the water levels decrease from elevation 217 feet to approximately 209 feet in well FC-2D which is screened in Confining Unit IIA-IIB (the Santee Formation above the Caw Caw Clay unit). Across the Caw Caw Clay, there is a drop in head of approximately 65 feet between well FC-2D (elevation 209 feet) and well FC-2C (elevation 145 feet) which is completed in the upper Congaree Formation of Aquifer Unit IIA.

The downward hydraulic gradient during July 1987 was calculated to range from 0.07 to 0.33 from the water table aquifer(Aquifer Unit IIB) to the upper Congaree Formation (Aquifer Unit IIA). There is a slight head reversal (increase in head) of about 2 feet between Aquifer Unit IIA and the upper portion of Confining System I-II (Wells FC-2B and FC-2A, respectively). There is a significant head reversal of 26 feet between the upper and lower portions of 


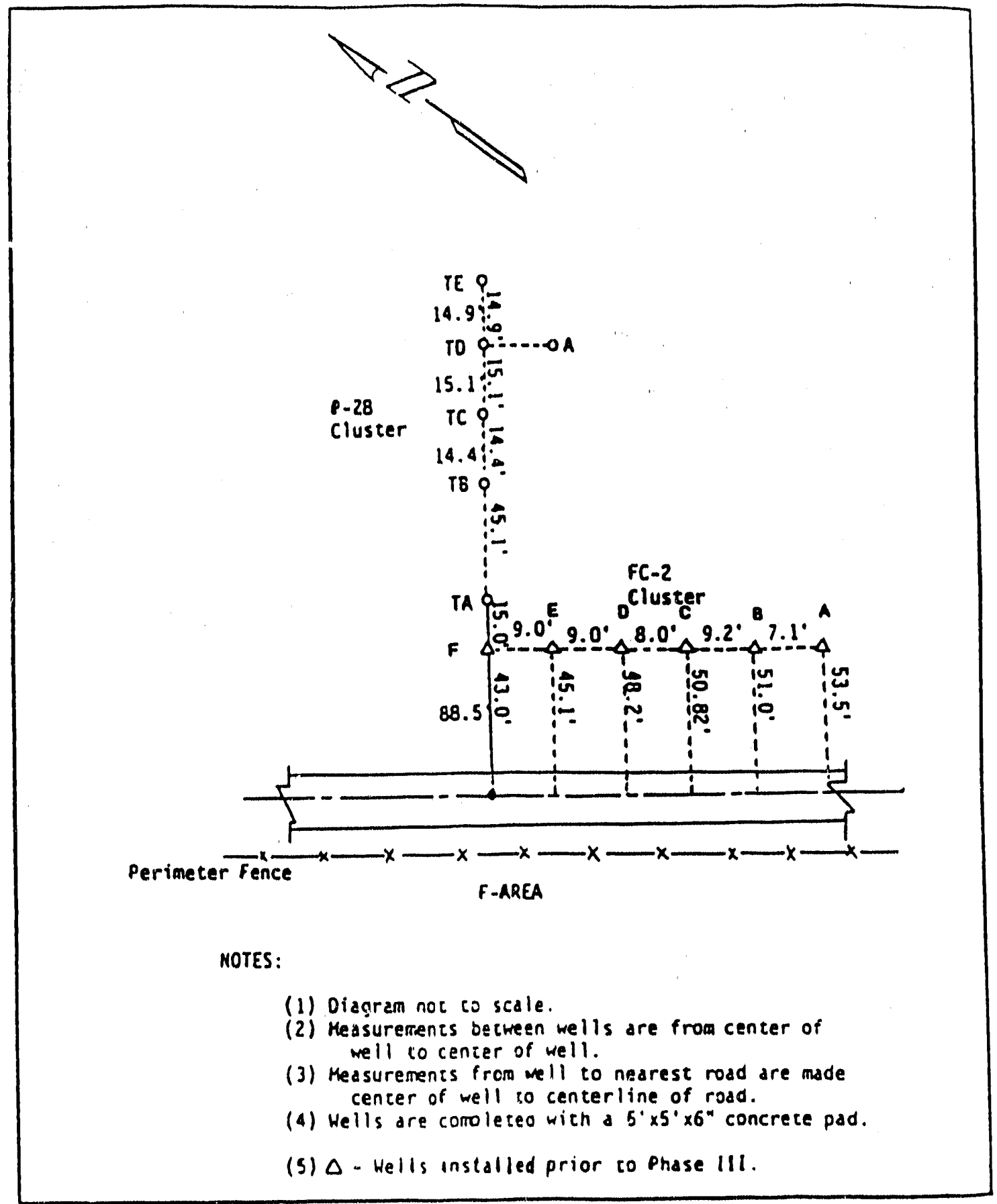

From DPST-88-627, E.I. du Pont de Nemours and Company, $1988^{\circ}$

FIGURE 2-7 LAYOUT MAP FOR WELL CLUSTERS P-28 AND FC-2 


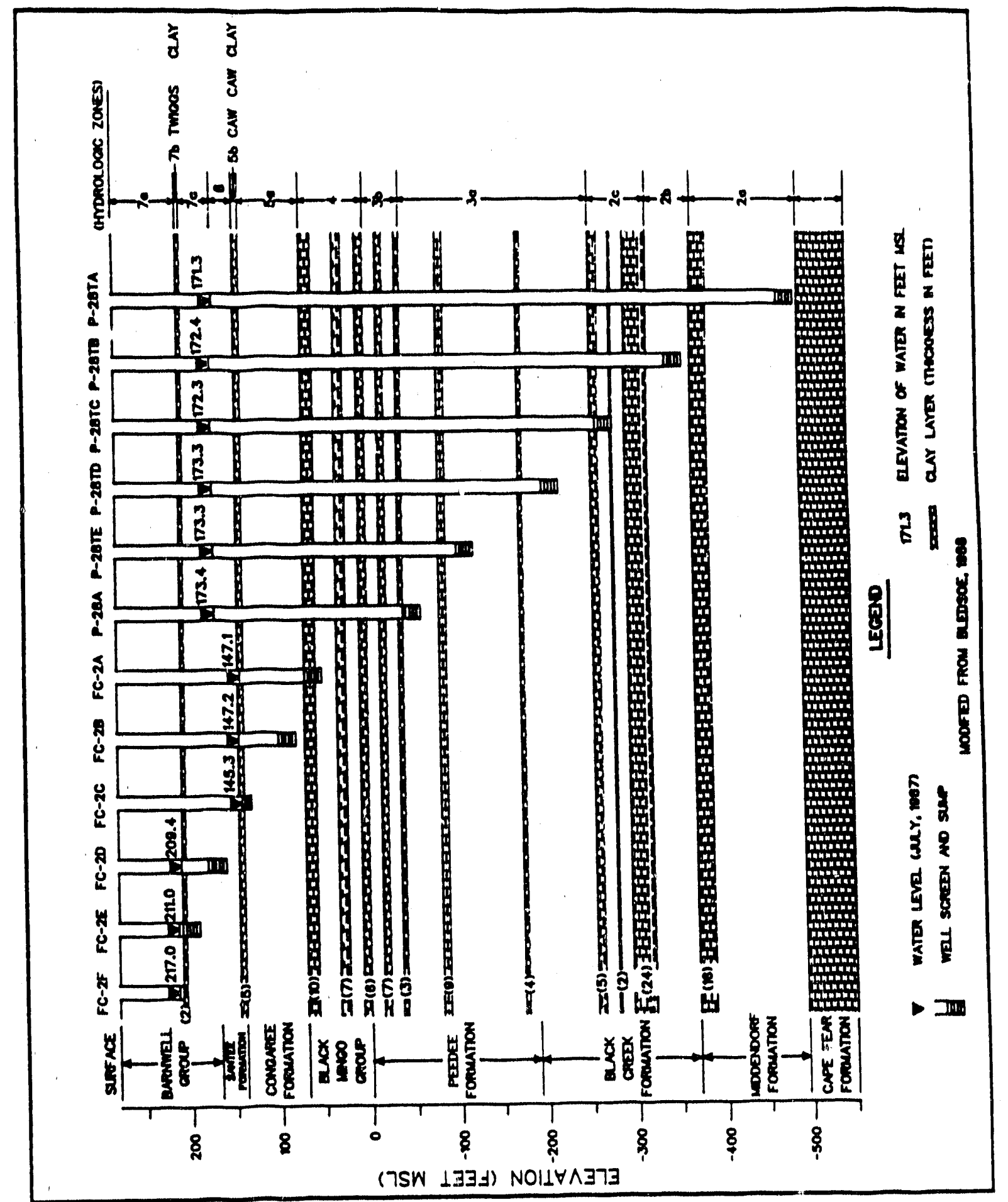

Modified from Bledsoe, $1988^{9}$

FIGURE 2-8 HYDRAULIC HEADS IN GROUNDWATER AT WELL CLUSTER P-28/FC-2 
Confining System I-II (Wells FC-2a and P-23A screened in the Willamsburg Formation and the upper Pee Dee Formation, respectively). Upward hydraulic gradients ranged from 0.04 to 0.24 . Below the Pee Dee, the head decreases slightly with increasing depth within Aquifer System I down to the lower Middendorf Formation (Well P-28TA) where the plezometric surface is found at an elevation of about 171 feet.

As shown on Figure 2-9, Upper Three Runs Creok acts as a regional discharge area for groundwater within Aquifer Unit IIA. The characteristics of the potentiometric surface contours near Upper Three Runs Creek indicate that any hazardous constituents which reach Unit IIA would not go any deeper, but would instead be transported laterally to Upper Three Runs Creek within Unit IIA.

Hydraulic heads in Aquifer Units IA and IB are controlled by recharge and discharge regions to the northeast and southwest, respectively, of SRS5. Figure 2-10 shows the regional relationship between hydraulic heads in Units IB and IIA. In the northwest and east-central pans of SRS, hydraulic heads in Unit IIA are higher than heads in Unit IB. Elsewhere at SRS hydraulic heads in Unit IB are higher than hydraulic heads in Unit IIA. Comparisons of hydraulic heads in the various zones within Unit IA with the hydraulic head in Unit IIA yield similar results.

Because of the lack of control between well clusters, the exact location of the line separating the region of head reversal from that of no head reversal is uncertain. As shown on Figure 2-10, a head reversal is believed to exist at F. Area Railroad Crosstie Pile, preventing downward migration of contaminants from Unit IIA through Confining System I-Il. However, if a head reversal does not exist at the unit, the rate of downward migration of groundwater from Unit IIA to Unit IB may be estimated from the parameter values applicable to Confining System I-II. Recently published data (April 199110) suggests that vertical hydraulic conductivity for Confining System I-II ranged from $7.98 \times 10^{-10} \mathrm{~cm} / \mathrm{sec}$ to $2.27 \times 10^{-8} \mathrm{~cm} / \mathrm{sec}$ at Site $B$ northeast of F-Area. This translates, conservatively, to $6.4 \times 10^{-5} \mathrm{ft} /$ day. The head change and hydraulic gradient across the confining system may be estimated from published data. A 


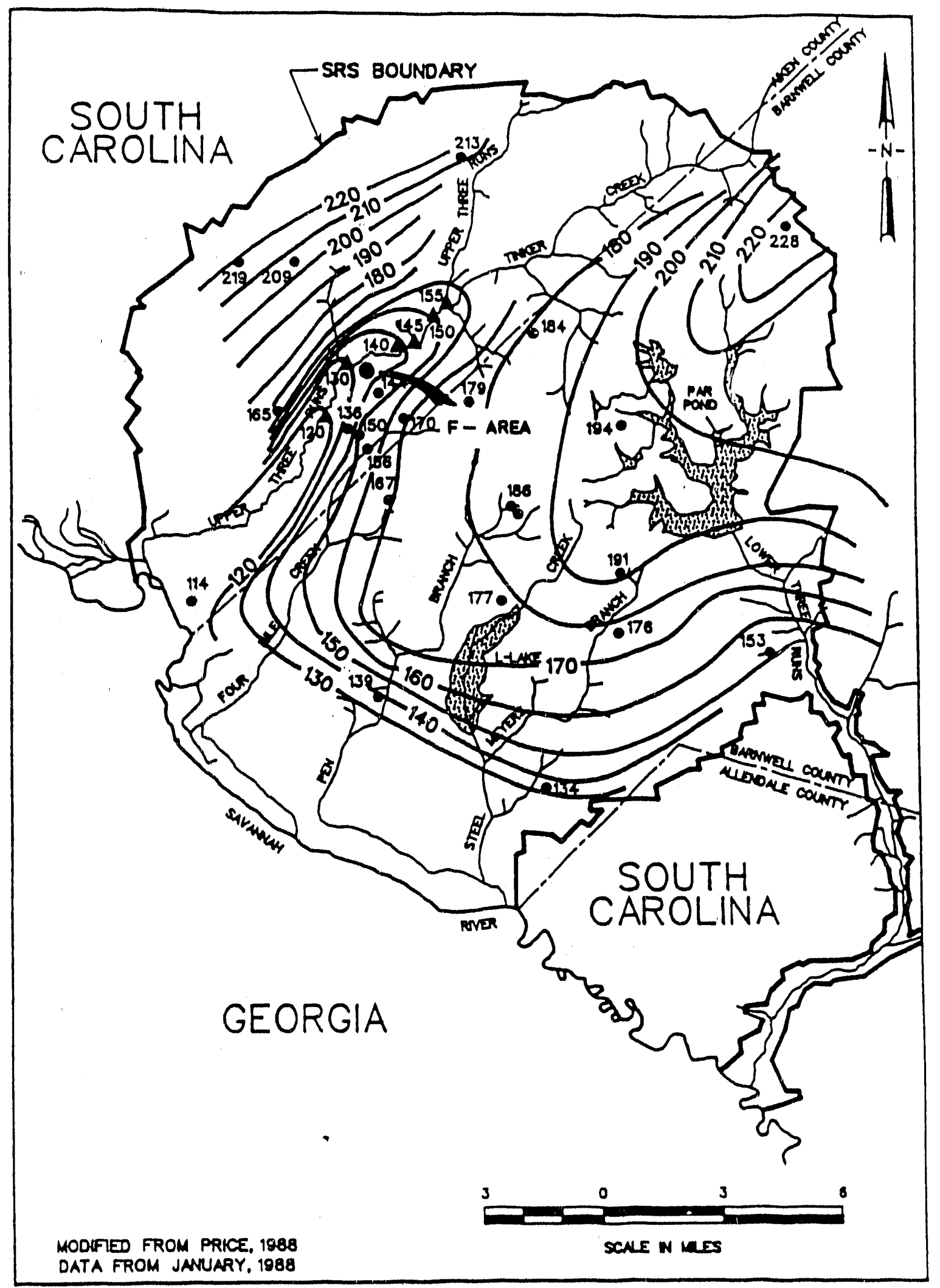

FIGURE 2-9 REGIONAL POTENTIOMETRIC SURFACE MAP, AQUIFER UNIT IIA Elevations in feet $\mathrm{msl}$ 


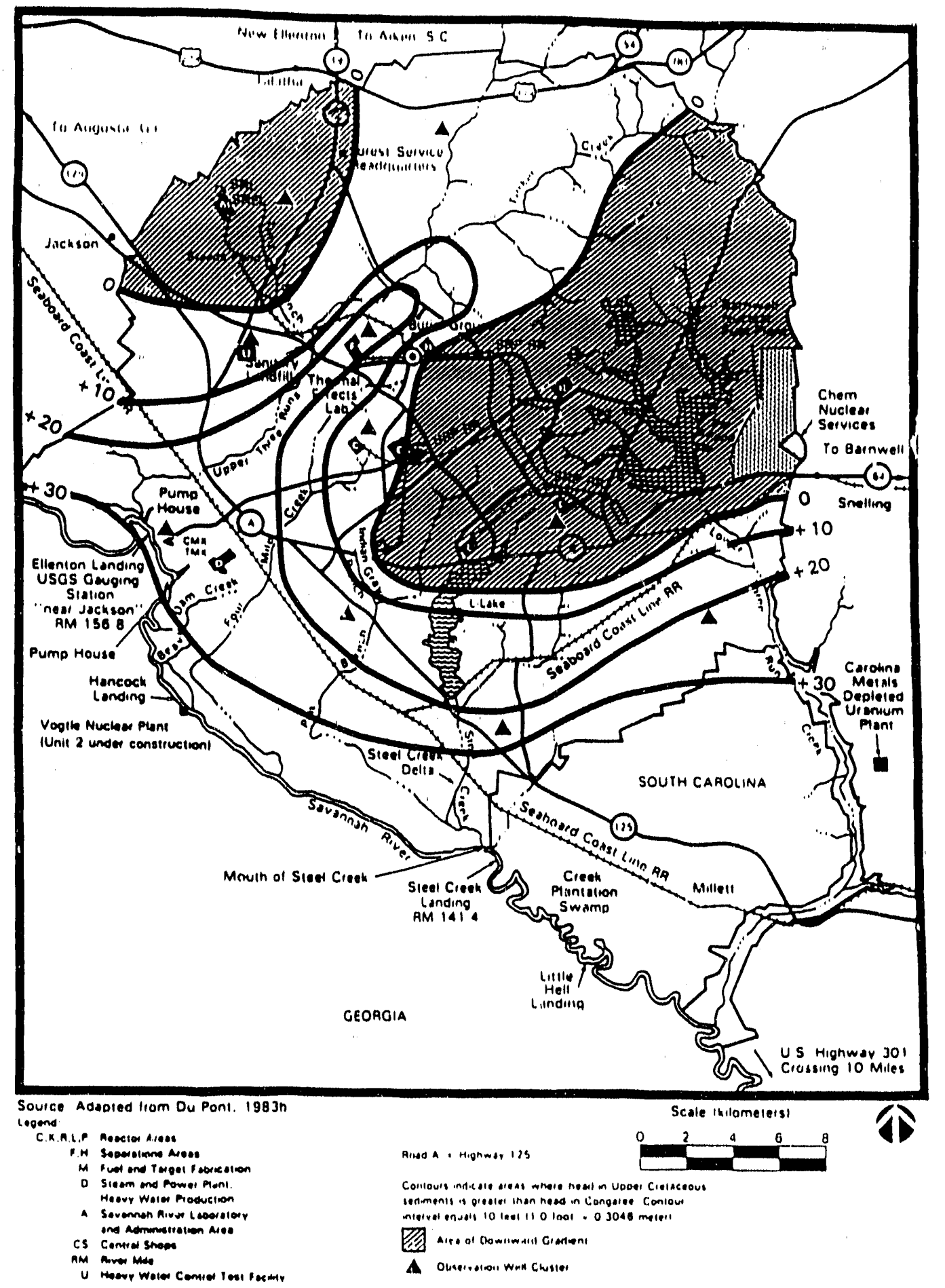

Source: Waste Management Actlvitles for Ground Water Protection, Savannah River Plant, Alken, South Carolina, Volume 2, DOE/EIS-0120, US Department of Energy, December 1987. 
maximum head change value of 12.5 feet, ylelding a hydraulic gradient of 0.11 $\mathrm{H} / \mathrm{ft}$, was reported by Bledsoe for Well Cluster P-179.

$$
\begin{aligned}
& K_{v}=\quad \text { vertical hydraulic conductivity }=6.4 \times 10^{-5} \mathrm{ft} / \text { day } 10 \\
& n_{\theta}=\quad \text { effective porosity (minimum, estimate) }=0.07^{11}
\end{aligned}
$$

Head change, Unit IIA to Unit IB (maximum) $=12.5$ feot (well cluster P-179) Saturated thickness of Confining System I-II (minimum, estimate) $=20$ feet 12 $i=$ hydraulic gradient $=0.11 \mathrm{f} / \mathrm{tt}$

Using Darcy's Law:

$$
V=K_{v} / n_{\theta}
$$

A velocity of $1.04 \times 10^{-4} \mathrm{ft} /$ day is obtained for groundwater leaking downward through Confining System I-ll. Assuming constant parameter values and continuity of the aquitard, it would require about 544 years for water to migrate from Aquifer Unit IIA through Confining System I-II into Aquifer Unit IB.

Based on the above information, it is unlikely that hazardous constituents that might be released from the F-Area Railroad Crosstie Pile would reach waterbearing strata (Units IB or IA) below A zuifer Unit IIA. Constituents entering the water table aquifer would instead be transported laterally at each clay layer within Aquifer IIB toward Upper Three Runs Creek, or would leak though Confining Unit \|A-\|B, and reach Upper Three Runs Creek through Aquifer Unit IIA.

In the general F-Area, the water table occurs within the Barnwell Group. Figure 2-3 shows this to be one of the younger (therefore, stratigraphically higher) units in the section. At higher topographic elevations in F-Area, these younger units exist and the water table occurs in them. At the lower elevations, the younger units are eroded and the water table resides in geologic units lower in the section. The deepest erosion (at Upper Three Runs Creek) exposes the Congaree Formation and, in this portion of F-Area, the water table occurs in this 
formation. These are textbook geomorphic features which have been observed in field reconnaissance, and are depicted on Figure 2-11.

Hydraulic conductivity obtained from a Steady-State Model Calibration for the Barnwell Group was $0.2-1.1 \mathrm{~m} /$ day. ${ }^{12}$ This value was determined from slug test data collected from nuarby F. and H-Area wells. Total porosity of the sediments that compose the Barnwell Formation range approximately from $35 \%$ to $60 \% .^{14}$ Effective porosity has been estimated at a lower value of approximately $20 \% 12$.

The horizontal hydraulic gradient of the water table (Barnwell Group) in the area of the railroad crosstie pile (between the four monitoring wells) is approximately 0.024 (calculated from 4th quarter 1988 data). The horizontal hydraulic gradient in the underlying Congaree Formation in the general F-Area is approximately $0.006 .^{12}$ The Congaree aquifer flow is toward Upper Three Runs Creok. ${ }^{12}$ 


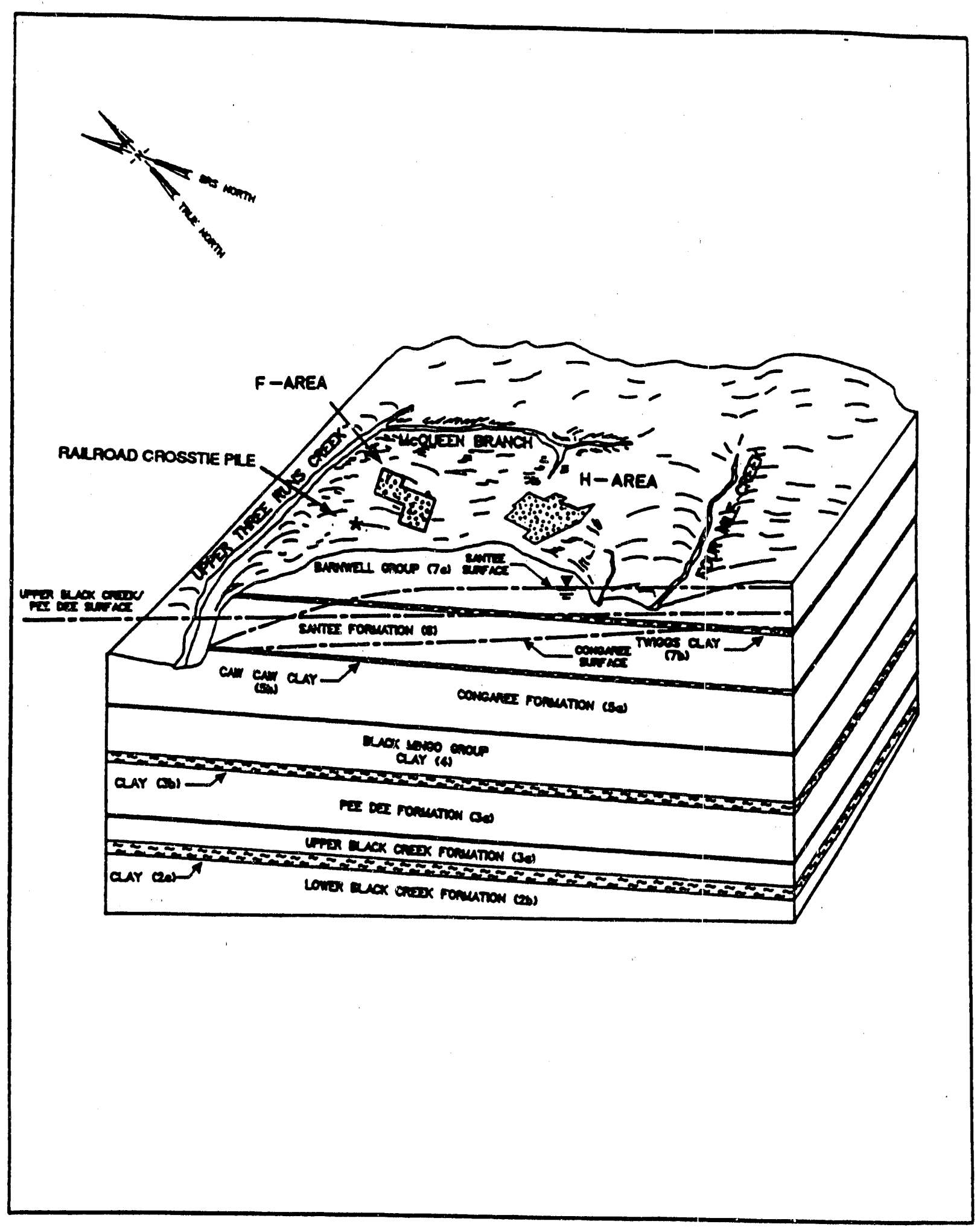

Modified from Diffield, et al., 198612

FIGURE 2-11 RELATIONSHIP BETWEEN PHYSIOGRAPHY AND HYDROSTRATIGRAPHY NEAR F-AND H-AREAS 


\subsection{SUMMARY REPORT OF FIELD OPERATIONS}

\subsection{Field Activities Summary}

Field activities for the Preliminary Characterization of the F-Area Railroad Crosstie site commenced March 12, 1991 and were completed March 22, 1991. Four soil borings, FRT-1HP, FRT-3HP, FRT-4HP and FRT-5HP were drilled and sampled initially to depths of $21.5 \mathrm{ft}$, for soil assessment purposes. Three of these borings, FRT-3HP, FRT-4HP, and FRT-5HP were then extended to the water table at a depth of about $100 \mathrm{ft}$ for collection of groundwater samples with the new version of the Hydropunch ${ }^{\mathrm{TM}}$, designated Hydropunch ${ }^{\mathrm{TM}}$ II. FRT-5HP is located in a wooded area upgradient from the railroad crosstie pile, and FRT3HP and -4HP were located downgradient. The borings were drilled at locations that had previously been staked and approved by DHEC. Boring locations are shown on Figure 2-6.

Drilling and sampling commenced March 12, 1991 at FRT-1HP and resumed after a one-day rain delay at the upgradient boring, FRT-5HP. The soil samples were collected from these two borings without incident. However, upon drilling FRT-5HP to the water table and driving the Hydropunch ${ }^{\mathrm{TM}}$ |I into position for sampling at the phreatic surface, it was determined that groundwater samples could not be collected with the system, as configured. The Hydropunch ${ }^{\text {TM }}$ II may be assembled in two different configurations; the standard configuration for sampling below the water table, and a new configuration for determining if lowdensity contaminants exist at the phreatic surface. The modified version of the Hydropunch ${ }^{\mathrm{TM}}$ simplifies disassembly, decontamination, and reassembly over previous versions. In addition, it is very rugged and is driven into position with a $140 \mathrm{lb}$ hammer in the same manner as a split spoon. After the Hydropunch ${ }^{\mathrm{M}}$ II is driven into sampling position, the driller retracts the body of the sampling device to expose a screen and allow groundwater to enter the sample chamber. In the standard configuration, water is retained in the sample chamber by a pair of check valves and the Hydropunch ${ }^{\mathrm{TM}}$ II is retrieved with a $200 \mathrm{ml}$ sample inside. However, in the water-table configuration, a bailer is lowered into the sample chamber through the drill rods. Therefore, it is possible to collect larger samples in the modified configuration by means of multiple trips with the bailer. 
Normal drilling rods are used in the standard configuration, with an AW adaptor in the drivehead. However, the system is designed to work with EW casing in the water-table configuration to enable passage of a 1 -in diameter bailer into the sample chamber.

The EW casing was not yet avallable for use at the Railroad Crosstie site, so it was necessary to sample through NWJ rods which have a 1.065-in ID constriction at each $10 \mathrm{ft}$. interval joint. This makes it very difficult to insert or retrieve the 1 -in. OD bailer. Also, since the top of the bailer is open, it is possible to introduce contamination into the bailer at each rod joint. In addition, the check valve in the bailer tends to unseat at each impact with a joint so that sample is lost in transit up the rods. This may not present a significant problem at a site where the water table is very shallow, but it does become significant where the water table is deeper. It may also be possible to use a smalldiameter top-fill bailer if the water depth in the sample chamber is sufficient, but a suitable bailer was not available.

After consultation with WSRC staff and a teleconference with QED, Inc., developer of the Hydropunch ${ }^{\mathrm{TM}}$, it was determined that EW casing would be necessary for successful completion of sampling with the Hydropunch ${ }^{\text {TM }}$ II, at this site. The Groundwater Monitoring Group of WSRC-EPD determined that it would not be feasible to procure EW casing within the project time frame. It was, therefore, necessary to either grout FRT-5HP or to use an alternative sampling procedure. The method selected by the Groundwater Monitoring Group of WSRC-EPD was to insert 2-in PVC casing and screen into each boring with a section of screen straddling the water table. A bailer was then used to collect a representative groundwater sample after the water level in each borehole stabilized.

A chronological summary of field activities is presented as Table 3-1, and soil sample data for each boring are presented as Tables 3-2 through 3-5. Sample data for groundwater samples from Borings FRT-3HP, 4HP, AND 5HP are presented as Table 3-6. 


\section{IABLE 3-1}

\section{F-Area Railroad Crosstle Slte \\ Chronologlcal Fleld Activitles Summary}

\section{DATE}

$3 / 11 / 91$
$3 / 12 / 91$
$3 / 13 / 91$

Friday $\quad 3 / 15 / 91$

Monday $\quad 3 / 18 / 91$

$3 / 19 / 91$

$3 / 20 / 91$

\section{REMARKS}

Prepare eqpt. - no sample containers available FRT-1HP; Drill and collect 7 soil samples to $21.5 \mathrm{ft}$.

Rain - shut down, shipped samples

3/14/91 FRT-5HP; Drill and collect 7 soil samples to $21.5 \mathrm{ft}$. - augered to $95 \mathrm{ft}$. - no water - no more augers, shut down for the day.

Auger to $105 \mathrm{ft}$., Adaptor sub to couple Hydropunch ${ }^{\mathrm{TM}}$ to rods won't fit. Mtg. Well Bldg. Telecom Bergren, QED, determine Hydropunch ${ }^{\mathrm{TM}}$ || sub is EW Casing thread - not rod thread.

No sub or EW casing available. Graves determines ID of NWJ rod couplings greater than 1 in., should allow passage of bailer. R. Hunter authorizes fabrication of NWJ box to EW casing pin adaptor. Telecom Tom Gaughan and Mary Flora. Elect to terminate drilling for the day rather than attempt to move to another boring. Grout FRT-1HP to surface.

New adaptor on site. Drive Hydropunch, trip in bailer with difficulty. Retrieve bailer with great difficulty and no sample - repeat 3 times. Sampling observed by Dan Wells, Dpt site - awaiting resolution.

Directed by J. Janssen to substitute 2 in. temporary piezometer for Hydropunch ${ }^{T M}$ sampling in FRT-5HP, $3 \mathrm{HP}$ and 4HP. Auger to $110 \mathrm{ft}$. and install 2 in. PVC piezometer in FRT-5HP.

FRT-4HP, drill and collect 7 soil samples to $21.5 \mathrm{ft}$., auger to $35 \mathrm{ft}$. Encountered large quartz cobbles during sampling requiring multiple sampling attempts at depths other than planned depths (se日 Table 4).

FRT-4HP (continued) Auger to 120 ft., set 2 in. PVC piezometer with $20 \mathrm{ft}$. screen. 
3/20/91 FRT-3HP, drill and collect 7 soil samples to $21.5 \mathrm{ft}$., auger to $25 \mathrm{ft}$.

3/21/91 FRT-3HP (continued) Auger to $105 \mathrm{ft}$, set 2 in. PVC piezometer with $15 \mathrm{ft}$. screen.

FRT -5HP - Collect water samples, 1145 to 1130.

FRT-4HP - Collect water samples, 1440 to 1640

FRT-3HP - Collect water samples, 1645 to 1840 including duplicate water sample labeled FRT-2HP.

Note: one liter metals samples filtered through 0.45 micron filter, except for sample labeled FRT-2HP which was unfiltered.

3/22/91 Remove PVC temporary piezometers and grout borings FRT-3HP, 4HP and 5HP to surface. 


\section{TABLE 3-2}

\section{Soll Sample Matrix, FRT-1HP}

Date: $3 / 12 / 91$

F-Area Railroad Crosstie Site

Technical Oversight: Charles B. Travis, PE, PG

Sampling Assistant: Sean W. Asquit'

Company: Sirrine Environmental Consultants

Sampling Station: FRT-1HP, Soil boring, 3 in. diameter split spoon

Preliminary SRS Coordinates: N 79,777.44; E 50,408.41

Preliminary Elevation:

Reference Point: $284.2 \mathrm{ft} \mathrm{msl}$

Station Established: $\quad 3 / 12 / 91$

SAMPLE INFORMATION:

\begin{tabular}{|c|c|c|c|c|c|}
\hline Sample ID & Depth & Date & Time & Sampler & Comments \\
\hline & & & & & \\
FRT-1HP-1 & $0-1.5$ & $3 / 12 / 91$ & 1400 & SWA & "O" ppm on OVA \\
FRT-1HP-2 & $1.5-3.0$ & $3 / 12 / 91$ & 1415 & CBT & "O" ppm on OVA \\
FRT-1HP-3 & $3.0-4.5$ & $3 / 12 / 91$ & 1440 & SWA & "O" ppm on OVA \\
FRT-1HP-4 & $4.5-6.0$ & $3 / 12 / 91$ & 1520 & SWA & "O" ppm on OVA \\
FRT-1HP-5 & $10.0-11.5$ & $3 / 12 / 91$ & 1550 & SWA & "O" ppm on OVA \\
FRT-1HP-6 & $15.0-16.5$ & $3 / 12 / 91$ & 1615 & SWA & "O" ppm on OVA \\
FRT-1HP-7 & $20.0-21.5$ & $3 / 12 / 91$ & 1650 & SWA & "O" ppm on OVA \\
& & & & & \\
\hline
\end{tabular}




\section{IABLE 3-3}

\section{Soll Sample Matrix, FRT-5HP}

Date: $\quad 3 / 14 / 91$

F-Area Rallroad Crosstio Site

Technical Oversight: $\quad$ Charles B. Travis, PE, PG

Sampling Assistant: Sean W. Asquith

Company: Sirrine Environmental Consultants

Sampling Station: FRT-5HP, Soil Boring, 3 in. diameter split spoon; Groundwater sampling station - see Table 6

Preliminary SRS Coordinates: N 79,823.00; E 50,701.78

Preliminary Elevation: $295.5 \mathrm{ft} \mathrm{msl}$

Reference Foint: Ground Surface

Station Established: $\quad 3 / 14 / 91$

\section{SAMPLE INFORMATION:}

\begin{tabular}{|c|c|c|c|c|c|}
\hline Sample ID & Depth & Date & Time & Sampler & Comments \\
\hline FRT-5HP-1 & $0.0-1.5$ & $3 / 14 / 91$ & 0900 & SWA & "O" ppm on HNu \\
FRT-5HP.-2 & $1.5-3.0$ & $3 / 14 / 91$ & 0950 & SWA & "O" ppm on HNu \\
FRT-5HP-3 & $3.0-4.5$ & $3 / 14 / 91$ & 1010 & SWA & "O" ppm on HNu \\
FRT-5HP-4 & $4.5-6.0$ & $3 / 14 / 91$ & 1040 & SWA & "O" ppm on HNu \\
FRT-5HP-5 & $10.0-11.5$ & $3 / 14 / 91$ & 1115 & SWA & "O" ppm on HNu \\
FRT-5HP-6 & $15.0-16.5$ & $3 / 14 / 91$ & 1145 & SWA & "O" ppm on HNu \\
FRT-5HP-7 & $20.0-21.5$ & $3 / 14 / 91$ & 1210 & SWA & "O" ppm on HNu \\
FRT-5HP-TB & & $3 / 14 / 91$ & 1245 & SWA & Trip Blank \\
& & & & & (Prepared by Lab) \\
\hline
\end{tabular}




\section{TABLE 3-4}

\section{Soll Sample Matrlx, FRT-4HP}

Date: $\quad 3 / 19 / 91$

F-Area Rallroad Crosstie Site

Technical Oversight: $\quad$ Charles B. Travis, PE, PG

Sampling Assistant: Sean W. Asquith

Company: Sirrine Environmental Consultants

Sampling Station: FRT-4HP, Soil Boring, 3 in. diameter split spoon

Groundwater Sampling Station - se日 Table 6

Preliminary SRS Coordinates: N 80,181.91; E 50,419.81

Preliminary Elevation: $283.6 \mathrm{ft} \mathrm{msl}$

Reference Point: Ground Surface

Station Established: $\quad 3 / 19 / 91$

\section{SAMPLE INFORMATION:}

\begin{tabular}{|l|c|c|c|c|l|}
\hline Sample ID & Depth & Date & Time & Sampler & \multicolumn{1}{|c|}{ Comments } \\
\hline FRT-4HP-1 & $0.0-1.25$ & $3 / 19 / 91$ & 1240 & CBT & "0" ppm on HNu \\
FRT-4HP-2 & $1.5-2.5$ & $3 / 19 / 91$ & 1320 & CBT & "0" ppm on HNu \\
FRT-4HP-3 & $3.0-4.0$ & $3 / 19 / 91$ & 1400 & CBT & 3 ppm top; \\
& & & & & "0" ppm at bit \\
FRT-4HP-4 & $4.5-6.0$ & $3 / 19 / 91$ & 1440 & CBT & 2 ppm on HNu \\
FRT-4HP-5 & $11.0-12.5$ & $3 / 19 / 91$ & 1530 & CBT & 2 ppm top; \\
& & & & "0" ppm at bit \\
FRT-4HP-6 & $16.0-17.0$ & $3 / 19 / 91$ & 1605 & CBT & "0" ppm \\
FRT-4HP-7 & $20.0-21.5$ & $3 / 19 / 91$ & 1635 & CBT & 2 ppm top; \\
& & & & & "O" ppm at bit \\
& & & & & \\
\hline
\end{tabular}




\section{TABLE 3-5.}

\section{Soll Sample Matrix, FRT-3HP}

Date: $3 / 20 / 91$

F-Area Railroad Crosstio Site

Technical Oversight: $\quad$ Charles B. Travis, PE, PG

Sampling Assistant: Sean W. Asquith

Company: Sirrine Environmental Consultants

Sampling Station: FRT-3HP, Soil Boring, 3 in. diameter split spoon;

Groundwater sampling station - see Table 6

Proliminary SRS Coordinates: N 79,872.19; E 50,387.93

Preliminary Elevation: $\quad 280.3 \mathrm{ft} \mathrm{msl}$

Reference Point: Ground Surface

Station Established: $\quad 3 / 20 / 91$

\section{SAMPLE INFORMATION:}

\begin{tabular}{|l|c|c|c|c|c|}
\hline Sample ID & Depth & Date & Time & Sampler & Comments \\
\hline FRT-3HP-1 & $0.0-1.5$ & $3 / 20 / 91$ & 1400 & CBT & "4" ppm on HNu \\
FRT-3HP-2 & $1.5-3.0$ & $3 / 20 / 91$ & 1425 & CBT & "2" ppm on HNu \\
FRT-3HP-3 & $3.0-4.5$ & $3 / 20 / 91$ & 1450 & CBT & "3" ppm on HNu \\
FRT-3HP-4 & $4.5-6.0$ & $3 / 20 / 91$ & 1525 & CBT & "2" ppm on HNu \\
FRT-3HP-5 & $10.0-11.5$ & $3 / 20 / 91$ & 1555 & CBT & "2" ppm on HNu \\
FRT-3HP-6 & $15.0-16.5$ & $3 / 20 / 91$ & 1645 & CBT & "4n ppm on HNu \\
FRT-3HP-7 & $20.0-21.5$ & $3 / 20 / 91$ & 1710 & CBT & "2" ppm on HNu \\
FRT-3HP-R & & $3 / 20 / 91$ & 1445 & SWA & Rinsate Blank \\
& & & & & \\
\hline
\end{tabular}




\section{TABLE 3-6}

\section{WATER SAMPLE MATRIX}

Date: 3/21/91 Water Samples

F-Area Railroad Crosstie Site

Technical Oversight: Charles B. Travis, PE, PG

Sampling Assistant: Sean W. Asquith

Company: Sirrine Environmental Consultants

Sampling Stations@Time: FRT-5HP@ 1145 to 1330

FRT-4HP@ 1535

FRT-3HP@ 1730

\begin{tabular}{|l|l|l|l|}
\hline & \multicolumn{1}{|c|}{ FRT-5HP } & \multicolumn{1}{|c|}{ FRT-4HP } & \multicolumn{1}{c|}{ FRT-3HP } \\
\hline Depth to Water & 103.0 feet & 97.4 fe日t & 93.9 fe日t \\
Date & $3 / 21 / 91$ & $3 / 21 / 91$ & $3 / 21 / 91$ \\
Time & $1145-1330$ & 1535 & 1730 \\
Volume Purged & 3 Bailers & 3 Bailers & 4 Bailers \\
pH & 5.39 & 5.11 & 5.53 \\
Specific Cond. & $65.2 \mu \mathrm{s} / \mathrm{cm}$ & $25.2 \mu \mathrm{s} / \mathrm{cm}$ & $26.4 \mu \mathrm{s} / \mathrm{cm}$ \\
Temperature & $19.0^{\circ} \mathrm{C}$ & $18.5^{\circ} \mathrm{C}$ & $18.5^{\circ} \mathrm{C}$ \\
Alkalinity & 4.4 & 2.2 & 2.2 \\
(mg/l as CaCO & & \\
& & & \\
& & & Collected Duplicate \\
& & & sample at time 1800 \\
& & & from FRT-3HP \\
& & & ID: FRT-2HP (did not \\
& & & \\
& & &
\end{tabular}




\subsection{Sample Collection, Handling, Packaging and Shlpping}

Samples were collected, handled, packaged and shipped in accordance with procedures specified in DPSOP $254^{15}$ and in the BE/RI Program Plan ${ }^{16}$. Samples were shipped from SRS by commercial carrier (overnight delivery) to Roy F. Weston Laboratories in Pennsylvania, using proper Chain of Custody procedures.

Sampling intervals and analyses were specified in the Program Plan (Tables 3$7 \& 3-8$ ), and Weston provided sample containers and coolers for shipping. The initial plan was to collect soll samples with a standard split spoon (1.375-in ID). However, the large quantity of soil required for analysis, and the cobbles encountered in the uppermost geologic formation, the Upland Unit, made it necessary to use a 3-in ID stainless steel split spoon.

All sampling equipment was decontaminated prior to use and between samples in accordance with the protocol developed for the RFV/RI program. The basic steps in this protocol are: 1) washing with an approved laboratory detergent, 2) steam cleaning, 3) rinsing with reagent-grade Isopropanol, and 4) rinsing with dionized (DI) water. All personnel who handled samples or sampling equipment wore Pylox sampling gloves, which were changed frequently to avoid cross-contamination of samples.

The borings were excavated with a truck-mounted drill and 6.25-in OD hollowstem augers to the specified depth, and the sampler lowered to the bottom of the augers on NWJ drill rods. A combination of hydraulic down pressure and a 140 pound hammer were then used to advance the sampler 24 inches, or to refusal. Upon recovery from the boring, the sampler was opened and the headspace monitored with an Organic Vapor Analyzer (OVA) or with an HNu photolonization detector (PID). The sample would then be split, and the entire length monitored with the $\mathrm{HNu}$. The sampling assistant would immediately collect and seal a soil sample for volatile organic analysis (VOA), where specifled. The remaining sample would then be blended in a stainless steel bowl, placed in sample containers provided by Weston, and packed on ice in coolers. The hydrogeologist used part of each sample to prepare a Field Geologic Log. A total of seven soll samples were collected from 


\section{TABLE 3-7}

\section{Soll Sampllno Intervals and Analyses}

$\begin{array}{ll}\text { Interval (t) } & \text { Analyses" } \\ 0-1.5 & 1,2,3,6 \\ 1.5-3.0 & 1,2,3,6 \\ 3.0-4.5 & 1,4,5,6,7 \\ 4.5-6.0 & 1,4,5,6,7 \\ 10-11.5 & 1,4,5,6,7 \\ 15-16.5 & 1,4,5,6,7 \\ 20-21.5 & 1,4,5,6,7\end{array}$

4 QAVC samples

- Speciflc analyses coded by group below.

\section{Soll parameters}

\begin{tabular}{|c|c|}
\hline $\begin{array}{l}\text { Group 1: } \\
\text { Group 1: } \\
\text { Group 1: }\end{array}$ & $\begin{array}{l}\text { Soll pH } \\
\text { Specifflc conductance of soil } \\
\text { Volatile organic vapor or soil gas } \\
\text { (field screening) }\end{array}$ \\
\hline $\begin{array}{l}\text { Group 1: } \\
\text { Group 2: } \\
\text { Group 3: } \\
\text { Group 4: } \\
\text { Group 5: } \\
\text { Group 6: }\end{array}$ & $\begin{array}{l}\text { Total organic carbon (TOC) } \\
\text { TCLP } \\
\text { Appendix IX constituents } \\
\text { Volatile organic analyses } \\
\text { Serni-volatile organic compounds } \\
\text { Radionuclide scan - gross alpha, } \\
\text { non-volatile beta, radium gamma PHA }\end{array}$ \\
\hline Group 7: & Metals listed on Appendix IX \\
\hline
\end{tabular}


IABLE 3-8

Groundwater Analyses"

$1,2,3,4,5,6$

2 QAVC samples

- Specific analyses coded by group below.

Field parameters

Group 1: $\quad \mathrm{pH}$

Group 1: $\quad$ Specific Conductance

Group 1: Temperature

Group 1: Field Alkalinity

\section{Laboratory parameters}

Group 2: $\quad \mathrm{pH}$

Group 2: $\quad$ Specific Conductance

Group 2: $\quad$ Temperature

Group 3: $\quad$ GC/MS scan (volatiles)

Group 4: Semi-volatile organic compounds

Group 5: Radionuclide scan:

gross alpha, non-volatile beta, radium, gamma PHA

Group 6: $\quad$ Appendix IX metals 
each test boring at specified intervals to a depth of 21.5 feet. Auger cuttings were logged below that depth to complete the Field Geologic Log. Therefore, the level of detail is significantly different in the segments of the logs above and below $21.5 \mathrm{ft}$. depth. The geologic logs are attached as Appendix $\mathrm{C}$.

Groundwater samples were collected March 21, 1991 from FRT-3HP, -4HP and $-5 \mathrm{HP}$. Samples were collected with a Teflon bailer through the 2-in PVC casing previously placed in each of these borings. The groundwater samples were also placed in containers provided by Weston and packed on ice in coolers. Specific conductance, $\mathrm{pH}$, temperature, and alkalinity were measured in the field and are recorded in Table 6. The samples designated for metals analysis were filtered in the field through 0.45 micron Nalgene disposable filier assemblies. A duplicate set of samples was collected from FHT-3HP and labeled as samples from FRT-2HP for QAQC purposes. FRT-2HP is a fictitious boring number as FRT-2HP was not drilled. The duplicate metals sample was not filtered in the field. Borings FRT-3HP, $-4 \mathrm{HP}$ and $-5 \mathrm{HP}$ were grouted to the surface March 22, 1991, following completion of groundwater sampling. In general, samples were shipped within 24 hours after collection. Chain of custody forms were filed at the Well Bullding, (772-7B) at SRS.

3.3 Quality Assurance/Quality Control

Technical oversight of drilling activities was provided by Sirrine Environmental Consultants, Inc.. Two persons were available for most sampling and oversight activities, a hydrogeologist and a sampling assistant. The technical oversight personnel had extensive experience with this type of project and were very familiar with SRS procedures and protocols. Test equipment was calibrated daily in accordance with procedures specified by the manufacturers. Organic vapor screening was performed with a model HW101 $\mathrm{HNu}$ with a 10.2 electron volt (EV) probe. Field parameters for groundwater samples were determined with a HyDAC conductivity, temperature, $\mathrm{pH}$ Tester manufactured by Cambridge Scientific Industries. Groundwater temperature was verified with a Taylor Model 21424-1 thermometer with a range from $-15^{\circ} \mathrm{C}$ to $105^{\circ} \mathrm{C}$. Alkalinity was determined in the field with a Hach Digital Titrator, Model Number 6900-01. 
QAQC samples were included with shipments as specified in the Program Plan. These consisted mostly of trip blanks provided by Weston. Also, a complete set of duplicate ground water samples was collected from boring FRT3HP and labeled with a fictitious boring number, FRT-2HP. The metals sample in the duplicate set was not filtered prior to shipment.

\subsection{Project Field Files}

Daily field activities were recorded in a bound journal as directed by the Groundwater Monitoring Group of WSRC-EPD. Photocopies of Daily Activity Reports and Field Geologic Logs are attached as Appendix C.

\subsection{UNIT SCREENING RESULTS}

\subsection{Data Development}

Laboratory analytical data from Weston arrived piecemeal over an extended period of time. The raw data was confusing and difficult to review. It was sent to Exploration Resources, Inc., a WSRC subcontractor in Athens, Georgia, for sorting and validation. Weston later sent the data to Exploration Resources on electronic media for sorting by computer. A printout of the test data, consisting of 295 single-spaced pages, was forwarded to Sirrine for review and incorporation into the report.

Review of the voluminous analytical data revealed that few analytes were reported above detection limits. Therefore, Exploration Resources used electronic sorting methods to produce tables that included only those analytes reported above detection limits. Summaries of soil sample analytical results are presented as Tables 4-1 through 4-6. Table 4-7 summarizes analytical data for the groundwater samples. A separate listing of analytes not detected was compiled in a Memo from Kathleen Gore to William Fay of Exploration Resources on August 23, 1991. That memo is included in this report as Appendix A. 


\subsection{Findings}

Based on data available, it is concluded that no hazardous substances have been released to the soils by the crosstie pile. Constituent values were compared with background values reported by Looney, et. al. 17 and with Drinking Water Standards (DWS). The soil series identified in the vicinity of the crosstie pile are the Blanton and Fuquay soil series and Udorthent soils. Udorthent is a generic term for sites where the natural soil horizons have been disrupted, usually by construction activities. The range of background soil values for each series is presented in Tables 4-8 through 4-10, along with the mean and standard deviation, where those can be calculated. In many instances, analytes were below detection limits, rendering mean and standard deviation calculations invalid.

\subsubsection{Metals in Soil Samples}

Constituent values for metals detected in soil samples at the unit are listed on Table 4-1. Metals are natural components of soils with widely varying concentrations. For example, a clean sand might have no detectables, while a clay or silt might be expected to have relatively high concentrations of various metals. The only metal detected at levels outside the range of background soil concentrations is copper. The range for copper in Blanton, Fuquay, and Udorthent soils is 0.36 to $11.1 \mathrm{mg} / \mathrm{kg}$ and three of the 28 values for copper exceeded this range. Those samples were: $11.7 \mathrm{mg} / \mathrm{kg}$ from a depth of 20 to 21.5 feet in FRT-1HP, $14.1 \mathrm{mg} / \mathrm{kg}$ from a depth of 10 to 11.5 feet in FRT-3HP, and $16.5 \mathrm{mg} / \mathrm{kg}$ from a depth of 15 to 16.5 feet in FRT-4HP. It is difficult to attribute these slightly elevated levels of copper to the railroad crosstie pile due to the depth at which they were encountered.

\subsubsection{Organic Analytes in Soil Samples}

Only three organic analytes were reported above detection limits in soil samples; acetone and pyridine from a depth 0.0 to 1.5 feet in FRT-3HP, and toluene in one or more samples in all borings. However, the acetone and pyridine were reported at the detection limit and toluene was reported at the detection limit of $6 \mathrm{mcg} / \mathrm{kg}$ in FRT-1HP and FRT-5HP. As shown on Table 4-2, 
toluene was reported at levels ranging from $12 \mathrm{mog} / \mathrm{kg}$ to $80 \mathrm{mcg} / \mathrm{kg}$ in FRT-3HP and FRT-4HP. These values are well above detection limits in two samples but well below the proposed DWS for toluene of $1,000 \mathrm{mcg} / \mathrm{L}$.

\subsubsection{Other Analytes in Soil Samples}

All parameters for percent solids, cyanide, sulfide, pH, specific conductance, and total organic carbon (TOC) were reported within the range of anticipated and background values for soil, as shown on Table 4-3.

\subsubsection{Metals in TCLP Samples}

Barium was reported in TCLP test results from all test borings (see Table 4-4). The highest reported value was $684 \mathrm{mcg} / \mathrm{l}$ for the sample collected at a depth of 1.5 to 3.0 feet in the background soil boring, FRT-5HP. Cadmium was reported in the TCLP test result on the soil sample from 0.0 to 1.5 feet in FRT-5HP at a level of $5.2 \mathrm{mcg} /$. That value is essentially at the detection limit of $5.0 \mathrm{mog} /$. These values are well below the allowable TCLP concentrations listed in 40 CFR 268.41, and also less than DWS of $1000 \mathrm{mcg} / \mathrm{l}$ and $10 \mathrm{mcg} / \mathrm{l}$, respectively.

\subsubsection{Radionuclides in Soil Samples}

Radionuclide activities in soil samples are listed on Table 4-5 and summarized on Table 4-6. Background values reported by Looney, et.al. ${ }^{17}$ and Fay and Pickett ${ }^{18}$ are included on Table 4-6 for comparison. Activities for gross alpha, non-volatile beta, and total radium are well above background in all but five of the 28 soil samples. Gross alpha and non-volatile beta appear to be significantly higher in the background boring, FRT-5HP, and in the "sidegradient" boring, FRT-1HP, than in the two "down-gradient" borings, FRT-3HP and FRT-4HP. However, the reverse appears to be true for total radium values. There do not appear to be any statistically significant trends in constituent activities with respect to sample depth. The test values do not fit a normal distribution, and therefore, a larger sample population would be needed for Analysis of Variance and determination of statistical significance. It is not possible at this time to attribute elevated soil radionuclide activities to the $\mathrm{F}$ Area Railroad Crosstie Pile. 


\subsubsection{Analytes Detected in Groundwater Samples}

Groundwater samples were collected from three test borings, FRT-3HP, 4HP, and 5HP. In addition, a replicate sample was collected from FRT-3HP. The replicate metals sample was not filtered in the field. Groundwater sample data are presented on Table 4-7. Drinking Water Standards (DWS) are summarized in Table 4-11. Gross alpha activities ranged from $150 \mathrm{pCi} / \mathrm{L}$ in FRT-3HP to 830 $\mathrm{pCl} / \mathrm{L}$ in FRT-5HP, well above DWS of $15 \mathrm{pCi} / \mathrm{L}$. Similarly, non-volatile beta activities ranged from $85 \mathrm{pCi} / \mathrm{L}$ in the replicate sample from FRT-3HP to 280 $\mathrm{pCl} / \mathrm{L}$ in FRT-4HP, again much higher than DWS of $50 \mathrm{pCi} / \mathrm{L}$. In addition, these activities are much higher than in the nearby FBP well series. Groundwater sample data from the third quarter of 1984 to the first quarter of 1991 are summarized in Appendix $B$ for the FBP wells. The radionuclides cesium-137 and total radium are well below DWS in all wells.

Reported values for specific conductance are about 100 times those measured in the field, and about 20 to 100 times those normally measured in the FBP wells. This indicates incorrect units of measurement may have been reported by the laboratory. Weston has not yet responded to inquiries on this issue.

Barium and chromium were reported above DWS in the unfiltered replicate sample from FRT-3HP, but below detection limits in the filtered sample.

The organic analytes tetrachloroethylene and trichloroethylene were detected above DWS in FRT-3HP and well above DWS in FRT-5HP along with carbon tetrachloride and trichloroflouromethane. No organic analytes were detected in FRT-4HP. The values reported for FRT-5HP are in line with those reported for the FBP wells which are upgradient toward F-Area.

The analytes detected in the groundwater downgradient from the unit cannot be attributed to the railroad crosstie pile as they are not significantly different than the values reported for the upgradient, background test boring. 
TABLE 4-1

Metals detected in F-Area Tie Yard soil samples

$\frac{\text { Core \# Depth Interval }}{\text { (feet) }} \frac{\text { Chromium }}{\mathrm{mg} / \mathrm{Kg}} \quad \frac{\text { Copper }}{\mathrm{mg} / \mathrm{Kg}} \quad \frac{\mathrm{Lead}}{\mathrm{mg} / \mathrm{Kg}} \quad \frac{\text { Mercury }}{\mathrm{mg} / \mathrm{Kg}} \quad \frac{\text { Vanadium }}{\mathrm{mg} / \mathrm{Kg}} \quad \frac{\text { Zlnc }}{\mathrm{mg} / \mathrm{Kg}}$

1

$0.0-1.50$
$1.5-3.00$
$3.0-4.50$
$4.5-6.00$
$10.0-11.50$
$15.0-16.50$
$20.0-21.50$

$\begin{array}{rr}3.9 & <4.5 \\ 9.8 & <4.8 \\ 7.3 & <5.0 \\ 8.5 & <4.8 \\ 6.9 & 6.8 \\ 6.5 & <4.6 \\ 6.8 & 11.7\end{array}$

1.70

0.14

$<9.1$

23.3

2.60

$<0.11$

$<9.6$

10.4

3.50

$<0.12$

16.0

16.1

4.7

$2.20<0.12$

$<0.11$

21.8

7.4

10.3

1.30

$<0.12$

18.2

6.7

1.10

11.3

12.0

3

$0.0-1.50$
$1.5-3.00$

$3.0-4.50$

$4.9<5.3$

2.70

$<0.12$

4.5

$3.6<5.1$

$4.4<5.1$

7.0

0.88

$<0.11$

10.8

$<10.2$

$<4.1$

0.82

$<0.12$

$<10.2$

$<4.1$

$4.5-6.00$

$<5.0$

4.30

0.16

12.9

20.1

$10.0-11.50$

$5.6 \quad 14.1$

5.20

$<0.12$

10.6

29.5

$15.0-16.50$

3.2
10.0

$<4.7$

1.5
1.40

$<0.12$

$<9.4$

5.3

4

$$
0.0-1.50
$$

1.5-3.00

$3.0-4.50$

$13.7<5.3$

3.50

$<0.12$

4.30

$<0.11$

19.9

6.3

$4.5-6.00$

9.9

$<3.9$

4.80

$<0.12$

14.9

$<4.2$

10.0-11.50

$4.7 \quad 5.6$

1.10

$<0.11$

$<0.67$

$<0.11$

$<11.0$

3.6

15.0-16.50

0.88

$<0.11$

$<8.5$

$<3.9$

$3.1 \quad 16.5$

$20.0-21.50$

$<1.7$

$<4.3$

$<0.59$

$<0.11$

$<8.2$

6.1

7.2

13.0

5

$\begin{array}{lrr}0.0-1.50 & 2.4 & <3.9 \\ 1.5-3.00 & 2.2 & <3.8 \\ 3.0-4.50 & 12.6 & <4.8 \\ 4.5-6.00 & 5.8 & <4.6 \\ 10.0-11.50 & 3.5 & 8.0 \\ 15.0-16.50 & <2.1 & <5.3 \\ 20.0-21.50 & 2.2 & <4.9\end{array}$

1.80
0.75
3.10
1.20
1.20
0.85
1.50

$<0.11$

$<8.7$

4.0

$<0.10$

$<7.8$

5.2

$<0.12$

$<7.7$

5.3

$17.1 \quad 4.4$

$<0.12$

11.3

8.9

$<0.12$

$<10.2$

17.6

$<0.11$

$<10.6$

9.9

6.1

Note: Core $1=$ FRT-1HP, Core $3=$ FRT-3HP, Core $4=$ FRT-4HP, and Core $5=$ FRT $-5 H P$ Compiled by Exploration Resources, inc. 
Organic analytes detected in F.Area Tie Yard soil samples

\begin{tabular}{|c|c|c|c|c|}
\hline Core \# & $\frac{\text { Depth Interval }}{\text { (feet) }}$ & $\frac{\text { Acetone }}{\mu \mathrm{g} / \mathrm{Kg}}$ & $\frac{\text { Pyridine }}{\mu g / \mathrm{Kg}}$ & $\frac{\text { Toluene }}{\mu \mathrm{g} / \mathrm{Kg}}$ \\
\hline 1 & $\begin{array}{l}0.0-1.50 \\
1.5-3.00 \\
3.0-4.50 \\
4.5-6.00 \\
10.0-11.50 \\
15.0-16.50 \\
20.0-21.50\end{array}$ & $\begin{array}{l}\text { B } 22 \\
\text { B } 130 \\
\text { B } 92 \\
\text { B } 95 \\
\text { B } 70 \\
\text { B } 100 \\
\text { B } 150\end{array}$ & $\begin{array}{l}<350 \\
<370 \\
<410 \\
<380 \\
<400 \\
<400 \\
<380\end{array}$ & $\begin{array}{r}\quad 6 \\
<6 \\
<6 \\
<6 \\
<6 \\
<6 \\
<6 \\
\text { J } 3\end{array}$ \\
\hline 3 & $\begin{array}{l}0.0-1.50 \\
1.5-3.00 \\
3.0-4.50 \\
4.5-6.0 \\
10.0-11.50 \\
15.0-16.50 \\
20.0-21.50\end{array}$ & $\begin{array}{l}220 \\
\text { B } 56 \\
\text { B } 240 \\
\text { B } 340 \\
\text { B } 320 \\
\text { B } 400 \\
\text { B } 40\end{array}$ & $\begin{aligned} 390, & <360 \\
& <440 \\
& <380 \\
& <390 \\
& <380 \\
& <350 \\
& <370\end{aligned}$ & $\begin{array}{r}41 \\
80 \\
\mathrm{~J} 3 \\
\mathrm{~J} 5 \\
12 \\
\mathrm{~J} 18 \\
\mathrm{~J} 4\end{array}$ \\
\hline 4 & $\begin{array}{l}0.0-1.50 \\
1.5-3.00 \\
3.0-4.50 \\
4.5-6.00 \\
10.0-11.50 \\
15.0-16.50 \\
20.0-2150\end{array}$ & $\begin{array}{l}\text { B } 16 \\
\text { B } 3100 \\
\text { B } 110 \\
\text { B } 210 \\
\text { B } 130 \\
\text { B } 2800 \\
\text { B } 77\end{array}$ & $\begin{array}{l}<390 \\
<350 \\
<390 \\
<380 \\
<370 \\
<360 \\
<340\end{array}$ & $\begin{array}{l}12 \\
\mathrm{~J} 3 \\
\mathrm{~J} 2 \\
\mathrm{~J} 3 \\
<6 \\
\mathrm{~J} 5 \\
<6\end{array}$ \\
\hline 5 & $\begin{array}{l}0.0-1.50 \\
1.5-3.00 \\
3.0-4.50 \\
4.5-6.00 \\
10.0-11.50 \\
15.0-16.50 \\
20.0-21.50\end{array}$ & $\begin{array}{l}\text { B } 30 \\
\text { B } 5200 \\
\text { B } 5000 \\
\text { B } 220 \\
\text { B } 54 \\
\text { B } 56 \\
\text { B } 43\end{array}$ & $\begin{array}{l}<340 \\
<340 \\
<380 \\
<380 \\
<370 \\
<380 \\
<370\end{array}$ & $\begin{array}{r}<6 \\
\mathrm{~J} 1 \\
<6 \\
\mathrm{~J} 3 \\
6 \\
\mathrm{~J} 2 \\
\mathrm{~J} 5\end{array}$ \\
\hline
\end{tabular}

Note: Core $1=$ FRT-1HP, Core $3=$ FRT-3HP, Core $4=$ FRT-4HP, and Core $5=$ FRT $-5 H P$ " $\mathrm{B}^{\prime \prime}$ indicates analyte found in blank; " $\mathrm{J}$ " indicates estimated value below quantification level.

Complied by Exploration Resources, Inc. 
TABLE 4-3

Other analytes detected in F.Area Tie Yard soil samples

\begin{tabular}{|c|c|c|c|c|c|c|c|}
\hline Core \#\# & $\frac{\text { Depth interval }}{\text { (feet) }}$ & $\frac{\text { Solids }}{\%}$ & $\frac{\text { Cranide }}{\mathrm{mg} / \mathrm{Kg}}$ & $\frac{\text { Sulfide }}{\mathrm{mg} / \mathrm{kg}}$ & $\mathrm{DH}$ & $\frac{\text { Spec, Cond }}{\mu \mathrm{S} / \mathrm{cm}}$ & $\frac{T O C}{\%}$ \\
\hline 1 & $\begin{array}{l}0.0-1.50 \\
1.5-3.00 \\
3.0-4.50 \\
4.5-6.0 \\
10.0-11.50 \\
15.0-16.50 \\
20.0-21.50\end{array}$ & $\begin{array}{l}93.6 \\
87.7 \\
87.2 \\
87.8 \\
84.8 \\
85.4 \\
87.0\end{array}$ & $\begin{array}{l}<1.1 \\
<1.1\end{array}$ & $\begin{array}{l}<0.27 \\
<0.28\end{array}$ & $\begin{array}{l}5.6 \\
5.3 \\
5.0 \\
4.9 \\
5.0 \\
5.0 \\
5.1\end{array}$ & $\begin{array}{l}12.1 \\
8.6 \\
8.8 \\
9.0 \\
8.5 \\
9.6 \\
7.1\end{array}$ & $\begin{array}{l}0.18 \\
0.96 \\
1.70 \\
1.30 \\
1.50 \\
1.60 \\
1.30\end{array}$ \\
\hline 3 & $\begin{array}{l}0.0-1.50 \\
1.5-3.00 \\
3.0-4.50 \\
4.5-6.0 \\
10.0-11.50 \\
15.0-16.50 \\
20.0-21.50\end{array}$ & $\begin{array}{l}87.6 \\
86.0 \\
95.4 \\
81.8 \\
86.2 \\
86.8 \\
88.8\end{array}$ & $\begin{array}{l}<1.1 \\
<1.2\end{array}$ & $\begin{array}{l}<0.28 \\
<0.29\end{array}$ & $\begin{array}{l}4.5 \\
4.9 \\
5.1 \\
5.1 \\
5.0 \\
5.0 \\
6.3\end{array}$ & $\begin{array}{r}16.1 \\
15.8 \\
9.8 \\
7.8 \\
12.2 \\
10.2 \\
8.8\end{array}$ & $\begin{array}{l}1.70 \\
1.20 \\
0.83 \\
1.80 \\
1.00 \\
1.10 \\
0.96\end{array}$ \\
\hline 4 & $\begin{array}{l}0.0-1.50 \\
1.5-3.00 \\
3.0-4.50 \\
4.5-6.0 \\
10.0-11.50 \\
15.0-16.50 \\
20.0-21.50\end{array}$ & $\begin{array}{l}87.0 \\
91.6 \\
80.9 \\
90.9 \\
88.8 \\
90.8 \\
93.3\end{array}$ & $\begin{array}{l}<1.1 \\
<1.1\end{array}$ & $\begin{array}{l}<0.29 \\
<0.27\end{array}$ & $\begin{array}{l}5.0 \\
5.3 \\
4.9 \\
\\
5.2 \\
5.3 \\
5.2\end{array}$ & $\begin{array}{r}15.5 \\
13.7 \\
20.4 \\
\\
8.9 \\
10.9 \\
12.5\end{array}$ & $\begin{array}{l}2.40 \\
0.85 \\
1.50 \\
\\
0.99 \\
0.79 \\
0.23\end{array}$ \\
\hline 5 & $\begin{array}{l}0.0-1.50 \\
1.5-3.00 \\
3.0-4.50 \\
4.5-6.0 \\
10.0-11.50 \\
15.0-16.50 \\
20.0-21.50\end{array}$ & $\begin{array}{l}94.4 \\
95.5 \\
80.6 \\
85.7 \\
86.5 \\
86.8 \\
87.6\end{array}$ & $\begin{array}{l}<1.1 \\
<1.0\end{array}$ & $\begin{array}{r}<0.26 \\
0.30\end{array}$ & $\begin{array}{l}5.0 \\
5.2 \\
5.2 \\
5.0 \\
5.1 \\
5.0 \\
5.2\end{array}$ & \begin{tabular}{r|}
11.8 \\
11.4 \\
11.3 \\
8.2 \\
13.4 \\
8.9 \\
10.1
\end{tabular} & $\begin{array}{l}0.30 \\
0.01 \\
1.50 \\
1.50 \\
1.10 \\
1.40 \\
1.20\end{array}$ \\
\hline
\end{tabular}

Note: Core 1 - FRT-1HP, Core 3 = FRT-3Hr', Core $4=$ FRT-4HP, and Core $5=$ FRT-5HP Compiled by Exploration Resources, Inc. 
Metals detected in F-Area Tie Yard TCLP samples

\begin{tabular}{|c|c|c|c|}
\hline Core \# & $\frac{\text { Depth Interyal }}{\text { (feet) }}$ & $\frac{\text { Barium }}{\mu g / L}$ & $\frac{\text { Cadmium }}{\mu g / L}$ \\
\hline 1 & $\begin{array}{l}0.0-1.50 \\
1.5-3.00\end{array}$ & $\begin{array}{r}<200 \\
382\end{array}$ & $\begin{array}{l}<5.0 \\
<5.0\end{array}$ \\
\hline 3 & $\begin{array}{l}0.0-1.50 \\
1.5-3.00\end{array}$ & $\begin{array}{r}281 \\
<200\end{array}$ & $\begin{array}{l}<5.0 \\
<5.0\end{array}$ \\
\hline 4 & $\begin{array}{l}0.0-1.50 \\
1.5-3.00\end{array}$ & $\begin{array}{r}283 \\
<200\end{array}$ & $\begin{array}{l}<5.0 \\
<5.0\end{array}$ \\
\hline 5 & $\begin{array}{l}0.0-1.50 \\
1.5-3.00\end{array}$ & $\begin{array}{r}<200 \\
684\end{array}$ & $\begin{array}{r}5.2 \\
<5.0\end{array}$ \\
\hline
\end{tabular}

Note: Core $1=$ FRT-1HP, Core $3=$ FRT-3HP, Core $4=$ FRT-4HP, and Core $5=$ FRT-5HP Compiled by Exploration Resources, Inc. 
TABLE 4-5

Radionuclides detected in F-Area Tie Yard soil samples

\begin{tabular}{|c|c|c|c|c|c|c|}
\hline Core \# & $\frac{\text { Depth Interval }}{\text { (feet) }}$ & $\frac{\text { Gross Alpha }}{\mathrm{pCi/g}}$ & $\frac{\text { Nonvolatile Beta }}{\mathrm{pCl} / \mathrm{g}}$ & $\frac{\text { Cesium-137 }}{\mathrm{pCl} / \mathrm{g}}$ & $\frac{\text { Thorlum-232 }}{\mathrm{pCl} / \mathrm{g}}$ & $\frac{\text { Total Radlum }}{\mathrm{pCl} / \mathrm{g}}$ \\
\hline 1 & $\begin{array}{l}0.0-1.50 \\
1.5-3.00 \\
3.0-4.50 \\
4.5-6.00 \\
10.0-11.50 \\
15.0-16.50 \\
20.0-21.50\end{array}$ & $\begin{array}{r}50.0 \pm 12.0 \\
44.0 \pm 13.0 \\
31.0 \pm 12.0 \\
58.0 \pm 15.0 \\
48.0 \pm 12.0 \\
22.0 \pm 9.0 \\
36.0 \pm 12.0\end{array}$ & $\begin{array}{l}16.0 \pm 4.0 \\
20.0 \pm 4.0 \\
27.0 \pm 5.0 \\
11.0 \pm 4.0 \\
19.0 \pm 3.0 \\
20.0 \pm 5.0 \\
15.0 \pm 5.0\end{array}$ & & $\begin{array}{l}0.9 \pm 0.1 \\
1.5 \pm 0.3 \\
1.0 \pm 0.2 \\
1.1 \pm 0.2 \\
1.8 \pm 0.3 \\
0.8 \pm 0.4 \\
0.7 \pm 0.2\end{array}$ & $\begin{array}{l}3.7 \pm 0.3 \\
3.9 \pm 0.3 \\
1.9 \pm 0.3 \\
2.2 \pm 0.3 \\
4.3 \pm 0.4 \\
3.7 \pm 0.4 \\
2.6 \pm 0.3\end{array}$ \\
\hline 3 & $\begin{array}{l}0.0-1.50 \\
1.5-3.00 \\
3.0-4.50 \\
4.5-6.00 \\
10.0-11.50 \\
15.0-16.50 \\
20.0-21.50\end{array}$ & $\begin{array}{r}29.0 \pm 10.0 \\
26.0 \pm 9.0 \\
33.0 \pm 11.0 \\
22.0 \pm 8.0 \\
0.0 \pm 11.0 \\
20.0 \pm 9.0 \\
25.0 \pm 10.0\end{array}$ & $\begin{array}{r}9.8 \pm 3.1 \\
13.0 \pm 3.0 \\
9.6 \pm 4.3 \\
16.0 \pm 4.0 \\
10.0 \pm 4.0 \\
12.0 \pm 4.0 \\
28.0 \pm 4.0\end{array}$ & $\begin{array}{l}0.0 \pm 0.1 \\
0.0 \pm 0.1 \\
0.0 \pm 0.1 \\
0.0 \pm 0.0 \\
0.0 \pm 0.1 \\
0.0 \pm 0.2 \\
0.0 \pm 0.1\end{array}$ & $\begin{array}{l}0.0 \pm 0.2 \\
0.5 \pm 0.2 \\
0.3 \pm 0.2 \\
1.1 \pm 0.3 \\
1.1 \pm 0.2 \\
1.3 \pm 0.2 \\
1.3 \pm 0.2\end{array}$ & $\begin{array}{r}6.1 \pm 0.6 \\
5.4 \pm 0.5 \\
3.1 \pm 0.4 \\
7.4 \pm 1.3 \\
4.3 \pm 0.5 \\
6.0 \pm 0.5 \\
13.0 \pm 1.0\end{array}$ \\
\hline 4 & $\begin{array}{l}0.0-1.50 \\
1.5-3.00 \\
3.0-4.50 \\
4.5-6.00 \\
10.0-11.50 \\
15.0-16.50 \\
20.0-21.50\end{array}$ & $\begin{array}{r}54.0 \pm 13.0 \\
52.0 \pm 14.0 \\
49.0 \pm 13.0 \\
23.0 \pm 11.0 \\
20.0 \pm 10.0 \\
9.8 \pm 8.0 \\
0.0 \pm 9.7\end{array}$ & $\begin{array}{r}21.0 \pm 4.0 \\
20.0 \pm 4.0 \\
15.0 \pm 4.0 \\
16.0 \pm 4.0 \\
0.0 \pm 6.5 \\
13.0 \pm 4.0 \\
11.0 \pm 4.0\end{array}$ & $\begin{array}{l}0.2 \pm 0.2 \\
0.0 \pm 0.1 \\
0.0 \pm 0.2 \\
0.0 \pm 0.2 \\
0.0 \pm 0.1 \\
0.0 \pm 0.1 \\
0.0 \pm 0.2\end{array}$ & $\begin{array}{l}1.8 \pm 0.2 \\
1.4 \pm 0.2 \\
1.1 \pm 0.2 \\
1.3 \pm 0.3 \\
0.7 \pm 0.2 \\
1.0 \pm 0.2 \\
0.0 \pm 0.2\end{array}$ & $\begin{array}{r}11.0 \pm 1.0 \\
7.5 \pm 0.6 \\
12.0 \pm 1.0 \\
6.3 \pm 0.6 \\
4.9 \pm 0.5 \\
5.7 \pm 0.5 \\
7.2 \pm 0.6\end{array}$ \\
\hline 5 & $\begin{array}{l}0.0-1.50 \\
1.5-3.00 \\
3.0-4.50 \\
4.5-6.00 \\
10.0-11.50 \\
15.0-16.50 \\
20.0-21.50\end{array}$ & $\begin{array}{r}41.0 \pm 12.0 \\
46.0 \pm 14.0 \\
40 \pm 12 \\
34 \pm 11 \\
44 \pm 12 \\
45 \pm 13 \\
62 \pm 16\end{array}$ & $\begin{array}{l}25.0 \pm 5.0 \\
23.0 \pm 4.0 \\
34.0 \pm 6.0 \\
11.0 \pm 4.0 \\
22.0 \pm 5.0 \\
34.0 \pm 5.0 \\
24.0 \pm 5.0\end{array}$ & & $\begin{array}{l}0.9 \pm 0.1 \\
1.0 \pm 0.2 \\
1.3 \pm 0.2 \\
1.0 \pm 0.3 \\
1.4 \pm 0.2 \\
1.5 \pm 0.3 \\
2.1 \pm 0.3\end{array}$ & $\begin{array}{l}2.7 \pm 0.2 \\
2.7 \pm 0.2 \\
2.3 \pm 0.2 \\
1.1 \pm 0.2 \\
0.8 \pm 0.1 \\
2.2 \pm 0.2 \\
0.7 \pm 0.1\end{array}$ \\
\hline
\end{tabular}

Note: Core $1=$ FRT-1HP, Core $3=$ FRT-3HP, Core $4=$ FRT-4HP, and Core $5=$ FRT-5HP Compiled by Exploration Resources, Inc. 
Soil Sample Radionuclide Summary

F-Area Railroad Crosstie Pile

$\begin{array}{cccc}\begin{array}{c}\text { Boring } \\ \text { Gross Alpha, pCl/g }\end{array} & \text { Mean } & \underline{S} & \text { Sample Size } \\ \text { Background17 } & 7.14 & 2.6 & 168 \\ \text { FRT-1HP } & 41.3 & 12.34 & 7 \\ \text { FRT-3HP } & 22.1 & 10.67 & 7 \\ \text { FRT-4HP } & 29.7 & 21.90 & 7 \\ \text { ERT-5HP } & 44.6 & 8.68 & Z \\ \text { All Samples } & 34.4 & 16.32 & 28\end{array}$

Non-Volatile Beta, pCi/g

$\begin{array}{lccr}\text { Background17 } & 9.58 & 4.14 & 168 \\ \text { FRT-1HP } & 18.3 & 5.02 & 7 \\ \text { FRT-3HP } & 14.1 & 6.56 & 7 \\ \text { FRT-4HP } & 13.7 & 7.02 & 7 \\ \text { ERT-5HP } & 24.7 & 7.87 & 7 \\ \text { All Samples } & 17.7 & 7.76 & 28\end{array}$

Total Radium, p,Ci/gr

$\begin{array}{lllr}\text { Background"18 } & 0.89 & 0.35 & 18 \\ \text { FRT-1HP } & 3.2 & 0.94 & 7 \\ \text { FRT-3HP } & 6.5 & 3.19 & 7 \\ \text { FRT-4HP } & 7.8 & 2.69 & 7 \\ \text { ERT-5HP } & 1.8 & 0.89 & 7 \\ \text { All Samples } & 4.8 & 3.21 & 28\end{array}$

"Radium 226 only 
TABLE 4-7

GROUNDWATER ANALYSES

Radionuclides detectod in F.Aroa Tlo Yard water samplos

\begin{tabular}{|c|c|c|c|c|}
\hline Analyie & Core 3 & $\begin{array}{l}\text { Core } 3 \\
\text { Replicate }\end{array}$ & Cored & Core 5 \\
\hline $\begin{array}{l}\text { Gross Alpha }(p C l / L) \\
\text { Nonvolatlle Bela (pCl/L) } \\
\text { Ceslum-137 (pCl/L) } \\
\text { Tolal Radium (pCl/L) }\end{array}$ & $\begin{array}{l}150 \pm 30 \\
130 \pm 30 \\
0 \pm 18 \\
0.4 \pm 0.2\end{array}$ & $\begin{array}{l}300 \pm 70 \\
85 \pm 19 \\
0 \pm 30 \\
1.2 \pm 0.4\end{array}$ & $\begin{array}{l}830 \pm 110 \\
280 \pm 30 \\
0 \pm 15 \\
0.7 \pm 0.3\end{array}$ & $\begin{array}{l}360 \pm 80 \\
110 \pm 20 \\
16 \pm 14 \\
0.5 \pm 0.2\end{array}$ \\
\hline
\end{tabular}

Other Analyses of F.Area Tie Yard water samples

\begin{tabular}{|c|c|c|c|c|}
\hline Analise & Core 3 & $\begin{array}{l}\text { Core } 3 \\
\text { Replicale }\end{array}$ & Core 4 & Core 5 \\
\hline $\begin{array}{l}\text { Specific Conductance }(\mu \mathrm{S} / \mathrm{cm}) \\
\mathrm{pH}\end{array}$ & $\begin{array}{l}2220 \\
5.2\end{array}$ & $\begin{array}{l}2210 \\
5.1\end{array}$ & $\begin{array}{l}2010 \\
5.3\end{array}$ & $\begin{array}{l}5610 \\
5.9\end{array}$ \\
\hline
\end{tabular}

Metals Analytes detecled in F.Area Tle Yard water samples

\begin{tabular}{|c|c|c|c|c|}
\hline Analvie & $\begin{array}{l}\text { Core } 3 \\
\text { Elliered }\end{array}$ & $\begin{array}{l}\text { Core } 3 \\
\text { Replicate } \\
\text { Unfillered }\end{array}$ & $\begin{array}{l}\text { Core } 4 \\
\text { Elltered }\end{array}$ & $\begin{array}{l}\text { Core } 5 \\
\text { Eillered }\end{array}$ \\
\hline Barium $(\mu g / L)$ & $<200$ & 3490 & $=200$ & $<200$ \\
\hline Berjllium ( $\mu g / \Omega)$ & $<5$ & 17 & $<5$ & $<5$ \\
\hline Coball ( $\mu g / 2)$ & $<50$ & 421 & $<50$ & $<50$ \\
\hline Chromlum ( $\mu / L)$ & $<10$ & 91.1 & $<10$ & $<10$ \\
\hline Copper $(\mu g /)$ & $<25$ & 150 & $<25$ & $<25$ \\
\hline Lead $(\mu \mathrm{g} / \mathrm{L})$ & $<3$ & 15 & $<3$ & $<3$ \\
\hline Mercury $(\mu g / L)$ & $<0.2$ & 1.2 & $<0.2$ & $<0.2$ \\
\hline Nickel $(\mu \mathrm{g} / \mathrm{L})$ & $<40$ & 74.7 & $<40$ & $<40$ \\
\hline Silver $(\mu g / L)$ & $<10$ & 27.9 & $<10$ & $<10$ \\
\hline $\operatorname{Tin}(\mu \rho / \mathcal{L})$ & $<100$ & 121 & $<100$ & $<100$ \\
\hline Vanadium ( $\mu g / L)$ & $<50$ & 219 & $<50$ & $<50$ \\
\hline $\operatorname{Zinc}(\mu g / L)$ & 60.8 & 352 & 20.1 & $<20$ \\
\hline
\end{tabular}

Organic Analyles delected in F-Area Tle Yard water samples

\begin{tabular}{|c|c|c|c|c|}
\hline Analvic & Oare 3 & $\begin{array}{l}\text { Core } 3 \\
\text { Replicate }\end{array}$ & Core 4 & Core 5 \\
\hline Carbon Telrachloride $(\mu \mathrm{g} / \mathrm{L})$ & j3 & 33 & $<5$ & 10 \\
\hline Telrachloroethylene ( $\mu \mathrm{g} / \mathrm{L})$ & 10 & 10 & $<5$ & 46 \\
\hline Trichloroethylene ( $\mu \mathrm{g} / \mathrm{L})$ & 11 & 15 & $<5$ & 81 \\
\hline Trichlorofluoromeihane $(\mu \mathrm{g} / \mathrm{L})$ & 46 & 27 & $<5$ & 49 \\
\hline
\end{tabular}

Note: Core 1 - FRT-1HP, Core $3=$ FRT-3HP, Core $4-$ FRT-4HP, and Core $5=$ FRT-5HP "J" indicates estimated value below quantification level.

Complled by Exploration Resources, Ino. 
TABLE 4-8

\section{F-AREA RAILROAD CROSSTIE PILE VICINITY RANGE OF BACKGROUND SOIL CONCENTRATIONS}

\begin{tabular}{|c|c|c|c|c|}
\hline \multirow[b]{2}{*}{ Analyte } & \multirow[b]{2}{*}{ Unit } & \multicolumn{3}{|c|}{ Blanton Soll Serles } \\
\hline & & Bange & Mean & Deviation \\
\hline $\begin{array}{l}\text { Al } \\
\text { As }\end{array}$ & $\begin{array}{l}(u g / g) \\
(u g / g)\end{array}$ & $\begin{array}{c}715-27,512 \\
<0.5-7.05\end{array}$ & 9061 & 7265 \\
\hline $\begin{array}{l}\mathrm{Ba} \\
\mathrm{Cd}\end{array}$ & $\begin{array}{l}(u g / g) \\
(u g / g)\end{array}$ & $\begin{array}{c}2.4-31.6 \\
0.27-0.99\end{array}$ & 12.2 & 8.08 \\
\hline $\mathrm{Cr}$ & (ug/g) & $1.7-35.2$ & 12.7 & 9.92 \\
\hline $\begin{array}{l}\mathrm{Cu} \\
\mathrm{Fe}\end{array}$ & $\left.\begin{array}{l}u g / g \\
u g / g\end{array}\right)$ & $\begin{array}{c}0.36-8.2 \\
1200-37,740\end{array}$ & $\begin{array}{c}3.1 \\
10,394\end{array}$ & \\
\hline $\begin{array}{l}\mathrm{Pb} \\
\mathrm{Mg} \\
\mathrm{Mn}\end{array}$ & $\begin{array}{l}(u g / g) \\
(u g / g)\end{array}$ & $\begin{array}{c}0.68-7.84 \\
20.1-296.7\end{array}$ & $\begin{array}{l}3.9 \\
104.7\end{array}$ & $\begin{array}{l}2.07 \\
62.1\end{array}$ \\
\hline $\begin{array}{l}\mathrm{Mn} \\
\mathrm{Hg}\end{array}$ & $\begin{array}{l}(u g / g) \\
(u g / g)\end{array}$ & $\begin{array}{l}<1.6-138.5 \\
<0.1-0.25\end{array}$ & " & $"$ \\
\hline $\mathrm{Ni}$ & (ug/g) & $0.11-9.6$ & " & " \\
\hline $\begin{array}{l}K \\
S_{\theta}\end{array}$ & $\begin{array}{l}(u g / g) \\
(u g / g)\end{array}$ & $\begin{array}{l}<40-<800 \\
<0.2-<4.0\end{array}$ & " & " \\
\hline $\mathrm{Ag}$ & (ug/g) & $0.24-<1.0$ & " & * \\
\hline $\mathrm{Na}$ & (ug/g) & $20.1-109.4$ & 45.4 & 17.9 \\
\hline $\begin{array}{l}\mathrm{Zn} \\
\mathrm{Cl}\end{array}$ & $\begin{array}{l}(\mathrm{ug} / \mathrm{g}) \\
\mathrm{ug} / \mathrm{g})\end{array}$ & $\begin{array}{c}3.0-24.6 \\
<1.25-11.2\end{array}$ & 9.2 & 4.53 \\
\hline $\begin{array}{l}\text { Nitrate as } \\
\text { Nitrogen }\end{array}$ & $(u g / g)$ & $<0.5-11.6$ & " & " \\
\hline Sulfate & (ug/g) & $<5-18.2$ & " & " \\
\hline $\begin{array}{l}\text { TOC } \\
\text { Gross Alpha }\end{array}$ & $\begin{array}{c}\text { (ug/g) } \\
\text { (PCl/g) }\end{array}$ & $\begin{array}{c}270-8842 \\
2.9-10.0\end{array}$ & 1845 & 2102 \\
\hline $\begin{array}{l}\text { Nonvolatile } \\
\text { Beta }\end{array}$ & (PCV/g) & $<5.0-46$ & • & " \\
\hline Uranium & $(\mathrm{PCl} / \mathrm{g})$ & $<1-2.2$ & ״ & " \\
\hline
\end{tabular}

Derived from Looney, et al, 199017

Soll Intervals Sampled

0-6 in, 7-61in, 61-76 in. and 98-121in

Range includes values below detection limit. Mean and standard deviation calculations would not yleld valld results. 


\section{TABLE $4-9$}

\section{F-AREA RAILROAD CROSSTIE PILE VICINITY \\ RANGE OF BACKGROUND SOIL CONCENTRATIONS}

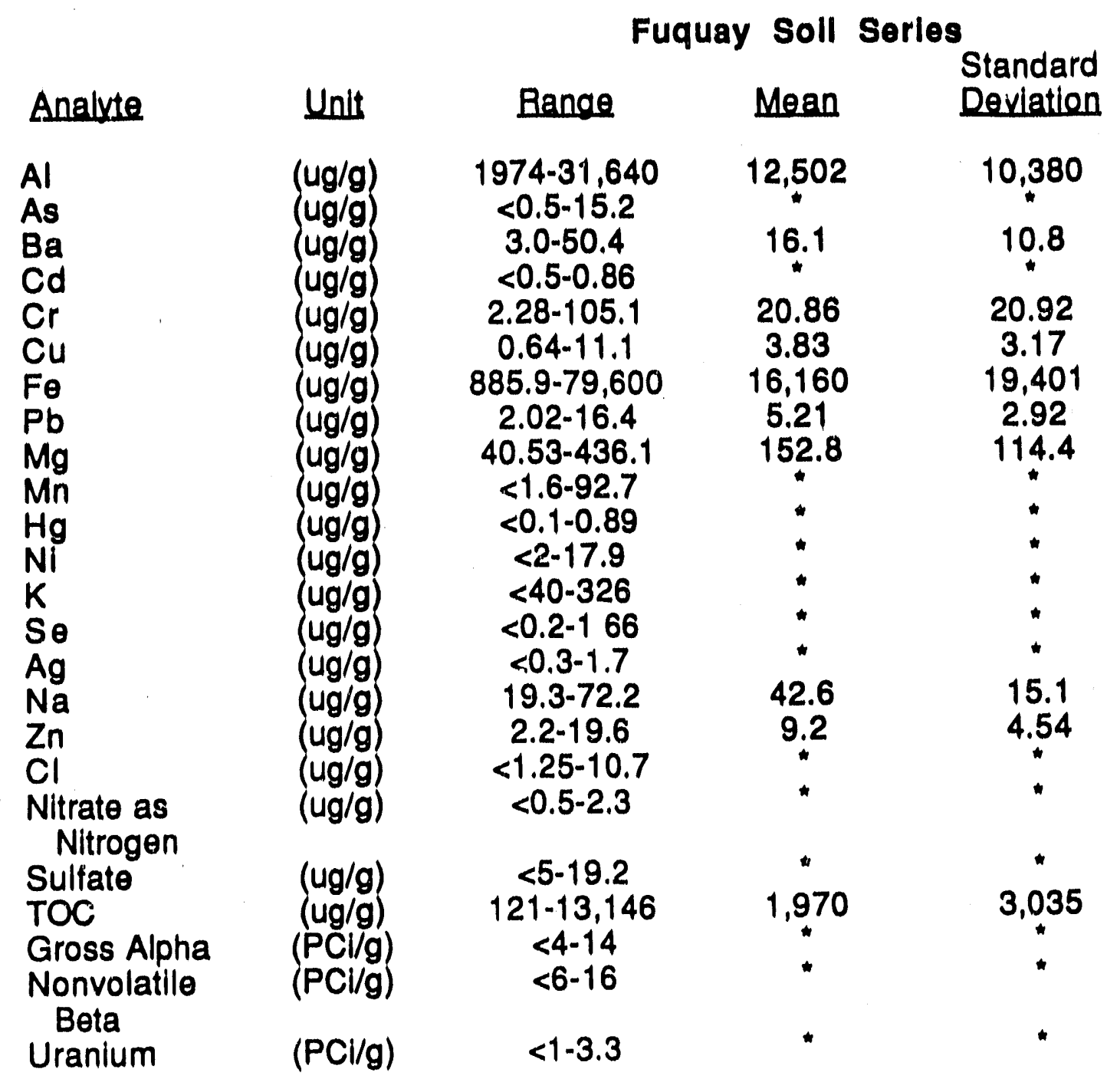

Derived from Looney, et al, 199017

Soil Intervals Sampled

0-6 in, 7-40 in, 40-84 in. and 120-132 in

Range includes values below detection limit. Mean and standard deviation calculations would not yield valid results. 
TABLE 4-10

F-AREA RAILROAD CROSSTIE PILE VICINITY

RANGE OF BACKGROUND SOIL CONCENTRATIONS

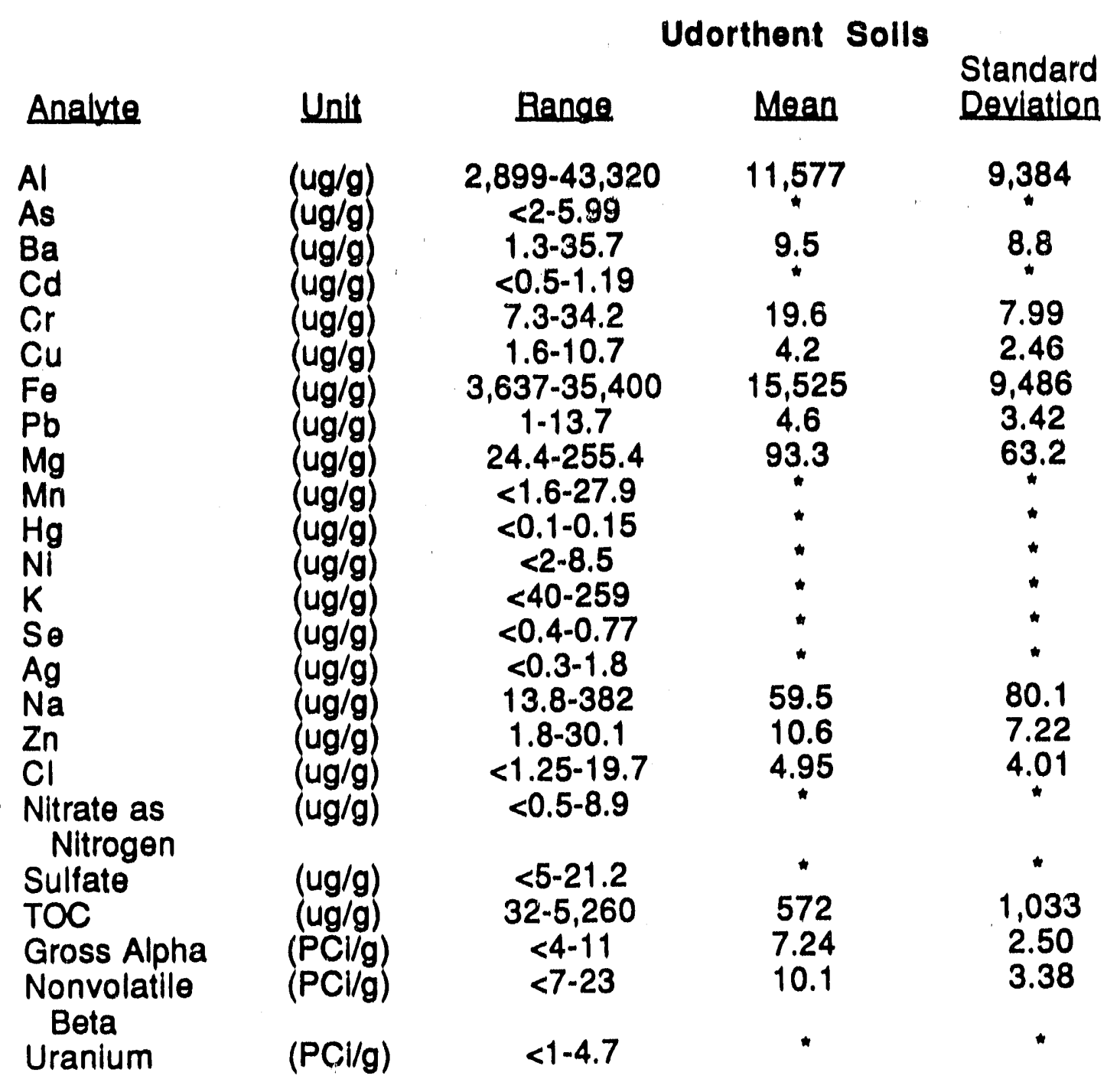

Derived from Looney, et al, 199017

Soll Intervals Sampled

0-24 in, 24-48 in, and 48-72 in

Range includes values below detection limit. Mean and standard deviation calculations would not yield valid results. 


\begin{tabular}{|c|c|c|c|c|}
\hline Analyte & Level & Units & Status & Reference \\
\hline Americium-241 & 4 & $\mathrm{pCi} / \mathrm{L}$ & proposed & EPA, 1986 \\
\hline Antimony-125 & 300 & $\mathrm{pCi} / \mathrm{L}$ & final & EPA, 1977 \\
\hline Arsenic & 0.05 & $\mathrm{mg} / \mathrm{L}$ & final & CFR, 1987 \\
\hline Barium & 1 & $\mathrm{mg} / \mathrm{L}$ & final & CFR, 1987 \\
\hline Barium-140 & 90 & $\mathrm{pCi} / \mathrm{L}$ & final & EPA, 1977 \\
\hline Benzene & 0.005 & $\mathrm{mg} / \mathrm{L}$ & final & EPA, 1987 \\
\hline Beryllium-7 & 6,000 & $\mathrm{pCi} / \mathrm{L}$ & final & EPA, 1977 \\
\hline Cadmium & 0.01 & $\mathrm{mg} / \mathrm{L}$ & final & CFR, 1987 \\
\hline Carbon-14 & 2,000 & $\mathrm{pCi} / \mathrm{L}$ & final & EPA, 1977 \\
\hline Carbon tetrachloride & 0.005 & $\mathrm{mg} / \mathrm{L}$ & final & EPA, 1987 \\
\hline Cesium-134 & 20,000 & $\mathrm{pCi} / \mathrm{L}$ & final & EPA, 1977 \\
\hline Cesium-137 & 200 & $\mathrm{pCi} / \mathrm{L}$ & final & EPA, 1977 \\
\hline Chlordane & 0.002 & $\mathrm{mg} / \mathrm{L}$ & proposed & EPA, 1989 \\
\hline $\begin{array}{l}\text { Chloroethene } \\
\text { (Vinyl chloride) }\end{array}$ & 0.002 & $\mathrm{mg} / \mathrm{L}$ & final & EPA, 1987 \\
\hline Chloroform" & 0.1 & mgll & final & CFR, 1987 \\
\hline Chromium & 0.05 & $\mathrm{mg} / \mathrm{L}$ & final & CFR, 1987 \\
\hline Chromium-51 & 6,000 & $\mathrm{pCi} / \mathrm{L}$ & final & EPA, 1977 \\
\hline Cobalt-58 & 9,000 & $\mathrm{pCi} / \mathrm{L}$ & final & EPA, 1977 \\
\hline Cobalt -60 & 100 & $\mathrm{pCi} / \mathrm{L}$ & final & EPA, 1977 \\
\hline Dibromochloropropane & 0.0002 & $\mathrm{mg} / \mathrm{L}$ & proposed & EPA, 1989 \\
\hline $\begin{array}{l}\text { 1,4-Dichlorobenzene } \\
\text { (p-Dichlorobenzene) }\end{array}$ & 0.075 & mg/L & final & EPA, 1987 \\
\hline 1,2-Dichloroethane & 0.005 & $\mathrm{mg} / \mathrm{L}$ & final & EPA, 1987 \\
\hline trans-1,2-Dichloroethene & & $\mathrm{mg} / \mathrm{L}$ & cosed & EPA, 1989 \\
\hline 1,1-Dichloroethylene & 0.007 & $\mathrm{mg} / \mathrm{L}$ & final & EPA, 1987 \\
\hline \multicolumn{5}{|c|}{ 2,4-Dichlorophenoxyacetic } \\
\hline acid & 0.1 & $\mathrm{mg} / \mathrm{L}$ & fin & CFr̆, 1987 \\
\hline 1,2-Dichloropropane & 0.005 & $\mathrm{mg} / \mathrm{L}$ & osed & EPA, 1989 \\
\hline Endrin & 0.0002 & $\mathrm{mg} / \mathrm{L}$ & final & CFR, 1987 \\
\hline Ethylbenzene & 0.7 & $\mathrm{mg} / \mathrm{L}$ & proposed & EPA, 1989 \\
\hline Fluoride & 4 & $\mathrm{mg} / \mathrm{L}$ & final & CFR, 1986 \\
\hline Gross alpha & 15 & $\mathrm{pCi} / \mathrm{L}$ & final & CFR, 1987 \\
\hline Heptachlor & 0.0004 & $\mathrm{mg} / \mathrm{L}$ & proposed & EPA, 1989 \\
\hline Heptachlor epoxide & 0.0002 & $\mathrm{mg} / \mathrm{L}$ & proposed & EPA, 1989 \\
\hline Iodine-129 & 1 & $\mathrm{pCi} / \mathrm{L}$ & tinal & EPA, 1977 \\
\hline Iodine-131 & 3 & $\mathrm{pCi} / \mathrm{L}$ & final & EPA, 1977 \\
\hline Iron-55 & 2,000 & $\mathrm{pCi} / \mathrm{L}$ & final & EPA, 1977 \\
\hline Irmn-59 & 200 & $\mathrm{pCi} / \mathrm{L}$ & final & EPA, 1977 \\
\hline Lead & 0.05 & $\mathrm{mg} / \mathrm{L}$ & final & CFR, 1987 \\
\hline Lindane & 0.004 & $\mathrm{mg} / \mathrm{L}$ & final & CFR, 1987 \\
\hline Manganese-54 & 300 & $\mathrm{pCi} / \mathrm{L}$ & final & EPA, 1977 \\
\hline Mercury & 0.002 & $\mathrm{mg} / \mathrm{L}$ & Gnal & CFR, 1987 \\
\hline Methoxychlor & 0.1 & $\mathrm{mg} / \mathrm{L}$ & final & CFR, 1987 \\
\hline Nickel-59 & 300 & $\mathrm{pCi} / \mathrm{L}$ & final & EPA, 1977 \\
\hline
\end{tabular}

\footnotetext{
- The level for total trihalomethanes is set at $0.1 \mathrm{mg} / \mathrm{L}$. Because bromated methanes are rarely detected in SRS groundwater, EHP presumes that most of the trihalomethanes present in site groundwater are chloroform.
} 


\begin{tabular}{|c|c|c|c|c|}
\hline Analyte & Level & Units & Status & Reference \\
\hline Nickel-63 & 50 & $\mathrm{pCi} / \mathrm{L}$ & final & EPA, 1977 \\
\hline Nitrate (as N) & 10 & $\mathrm{mg} / \mathrm{L}$ & final & CFR, 1987 \\
\hline Nitrite (as N) & 0.001 & $\mathrm{mg} / \mathrm{L}$ & proposed & EPA, 1989 \\
\hline Nonvolatile beta & 50 & $\mathrm{pCi} / \mathrm{L}$ & proposed & EPA, 1986 \\
\hline PCBs & 0.0005 & $\mathrm{mg} / \mathrm{L}$ & proposed & EPA, 1989 \\
\hline Pentachlorophenol & 0.2 & $\mathrm{mg} / \mathrm{L}$ & proposed & EPA, 1989 \\
\hline Potassium-40 & 300 & $\mathrm{pCi} / \mathrm{L}$ & proposed & EPA, 1986 \\
\hline Radium-226 & 4 & $\mathrm{pCi} / \mathrm{L}$ & proposed & EPA, 1986 \\
\hline Radium-228 & 8 & $\mathrm{pCi} / \mathrm{L}$ & proposed & EPA, 1986 \\
\hline Ruthenium-103 & 200 & $\mathrm{pCi} / \mathrm{L}$ & final & EPA, 1977 \\
\hline Ruthenium-106 & 30 & $\mathrm{pCi} / \mathrm{L}$ & final & EPA, 1977 \\
\hline Selenium & 0.01 & $\mathrm{mg} / \mathrm{L}$ & final & CFR, 1987 \\
\hline Silver & 0.05 & $\mathrm{mg} / \mathrm{L}$ & final & CFR, 1987 \\
\hline Silvex & 0.01 & $\mathrm{mg} / \mathrm{L}$ & final & CFR, 1987 \\
\hline Strontium-89 & $20^{b}$ & $\mathrm{pCi} / \mathrm{L}$ & final & EPA, 1977 \\
\hline Strontium-90 & 8 & $\mathrm{pCi} / \mathrm{L}$ & final & EPA, 1977 \\
\hline Styrene & 0.005 & $\mathrm{mg} / \mathrm{L}$ & proposed & EPA, 1989 \\
\hline Technetium.99 & 900 & $\mathrm{pCi} / \mathrm{L}$ & final & EPA, 1977 \\
\hline Tetrachloroethylene & 0.005 & $\mathrm{mg} / \mathrm{L}$ & proposed & EPA, 1989 \\
\hline Tin-113 & 4,000 & $\mathrm{pCi} / \mathrm{L}$ & proposed & EPA, 1986 \\
\hline Toluene & 2 & $\mathrm{mg} / \mathrm{L}$ & proposed & EPA, 1989 \\
\hline Total radium & 5 & $\mathrm{pCi} / \mathrm{L}$ & final & CFR, 1987 \\
\hline Toxaphene & 0.005 & $\mathrm{mg} / \mathrm{L}$ & final & CFR, 1987 \\
\hline 1,1,1-Trichloroethane & 0.2 & $\mathrm{mg} / \mathrm{L}$ & final & EPA, 1987 \\
\hline Trichloroethylene & 0.005 & $\mathrm{mg} / \mathrm{L}$ & final & EPA, 1987 \\
\hline Tritium & 20 & $\mathrm{pCi} / \mathrm{mL}$ & final & CFR, 1987 \\
\hline Uranium-234 & 28 & $\mathrm{pCi} / \mathrm{L}$ & proposed & EPA, 1986 \\
\hline Uranium-235 & 28 & $\mathrm{pCi} /$ & proposed & EPA, 1986 \\
\hline Uranium-238 & 28 & $\mathrm{pCi} / \mathrm{L}$ & proposed & EPA, 1986 \\
\hline Xylenes & 10 & $\mathrm{mg} / \mathrm{L}$ & proposed & EPA, 1989 \\
\hline Zinc -65 & 300 & $\mathrm{pCi} / \mathrm{L}$ & final & EPA, 1977 \\
\hline Zirconium- 95 & 200 & $\mathrm{pCi} / \mathrm{L}$ & final & EPA, 1977 \\
\hline
\end{tabular}

- This is the lower of two levels given for strontium-89.

\section{References:}

CFR (Code of Federal Regulations), 1986. "National Primary Drinking Water Regulations," 40 CFR, Part 141 , pp. 521.568 , Washington, DC.

CFR (Code of Federal Regulations), 1987. "National Primary Drinking Water Regulations," 40 CFR, Part 141, pp. 526-575, Washington, DC.

EPA (U.S. Environmental Protection Agency), 1977. National Interim Primary Drinking Water Regulations, EPA-570/9-76-003, Washington, DC.

EPA (U.S. Environmental Protection Agency), 1986. "Water Pollution Control; National Primary Drinking Water Regulations, Radionuclides (Proposed), ${ }^{n}$ Federal Register, September 30, 1986, pp. 34836-34862, Washington, DC.

EPA (U.S. Environmental Protection Agency), 1987. "National Primary Drinking Water Regulations; Syn. thetic Organic Chemicals; Monitoring for Unregulated Contaminants, "Federal Register, July 8, 1987, pp. 25690-25717, Washington, DC.

FPA (II S Envirnnmental Protection Agency), 1989. “National Primary and Secondary Drinking Water Regulations (Proposed Rule), Federal Register, May 22, 1989, pp. 22062-22160, Washington, DC. 


\section{BEFERENCES}

1. Bignell, D.T., WSRC, Railroad Crosstie Disposal Alternatives, Letter to Mr. Donald Gross, Jr, DOE.(January, 1991)

2. AAR, Toxicity Characteristic Leaching Procedure Testing of Railroad Crossties (R-708). Association of American Railroads, Chicago, IL (1988)

3. USDA, Soil Survey of Savannah River Plant Area. Parts of Aiken. Barnwell. And Allendale Counties, South Carolina, U. S. Department of Agriculture, Soil Conservation Service, (1990)

4. Aadland, R.K, and H.W. Bledsoe, Classification of Hydrostratigraphic Units at Savannah River Site. South Carolina. WSRC-RP-90-987, WSRC, Savannah River Laboratory, Aiken, SC (1990).

5. Price, V., "Subsurface Hydrogeology," in Stephenson, A.2., ed., Einal Safety Analysis Report for DWPF (Draft). DPSTSA-200-10, E.I. du Pont de Nemours and Company, Savannah River Plant, Aiken, SC (1988).

6. WSRC, Savannah River Site Environmental Report for 1988. WSRC-RP-89-59, Westinghouse Savannah River Company for the U.S. Department of Energy, Aiken, SC (1988).

7. Fallaw, W.C., Subsurface Stratigraphy and Structure of the A-and M-Area at the Savannah River Site Aiken, SC, WSRC (DRAFT, 1990);

8. Olson, C., Wells Monitored by the Health Protection Department. HPR-88-001, E.I. du Pont de Nemours and Company and Exploration Software, Savannah River Plant, Aiken, SC (1988).

9. Bledsoe, H.W., SRP Baseline Hydrogeologic Investigation - Phase III. DPST-88627, E.I. du Font de Nemours \& Co., Savannah River Laboratory, Aiken, SC (1988)

10. WSRC, Site Hydrogeolegic/Geotechnical Characterization Repert for Site B. New Municipal Soil Waste Landfill, WSRC-RP-91-392, Westinghouse Savannah River Company for the U.S. Department of Energy, Aiken, SC (1991).

11. Huber, L.A. and H.W. Bledsoe, Enyironmental Information Document Hydrofluoric Acid Spill Area. DPST-85-696, E.I. du Pont de Nemours and Company, Savannah River Laboratory, Aiken, SC (1987).

12. Duffield, G. M., D. R. Buss, R.W. Root, Jr., S.S. Hughes, and J.W. Mercer, Characterization of Groundwater Flow and Transport in the General Separations Areas. Savannah River Plant: Flow Model Refinement and Particle Tracking Analysis, CORR-86-0031, Geo Trans, Inc., for E.I. du Pont de Nemours and Company, Savannah River Laboratory, Aiken, SC (1986). 
13 USDOE, Final Environmental Impact Statement: Waste Management Activities for Groundwater Protection. Savannah River Plant. Alken. South Carolina. DOE/EIS-0120, U.S. Department of Energy, Savannah River Operations Office, Aiken, SC (1987).

14. Root, R.W., Jr. Groundwater Data from the H-Area Savannah Biver Plant. South Carolina. DPST-80-601, E.I. du Pont de Nemours and Company, Savannah River Laboratory, Aiken, SC (1980)

15. WSRC, Hydrogeologic Data Collection Methods. Procedures and Specifications. DPSOP 254, adapted by WSRC from E.I. du Pont de Nemours and Company, Savannah River Site, Aiken, SC (1987 or latest version).

16. WSRC, BCRA Facility Investigation/Remedial Investigation (RF/RI) Program Plan. WSRC-RP-89-994, Westinghouse Savannah River Company, Aiken, SC (1989)

17. Looney, B.B., C.A. Eddy, M. Ramdeen, J. Pickett, V. Rogers, M.T. Scott and P.A. Shirley, Geochemical and Physical Properties of Soils and Shallow Sediments at the Savannah River Site (u). WSRC-RP-90-1031, Westinghouse Savannah River Company, Alken, SC (1990).

18. Fay, W. M., and J. B. Pickett, Decumentation of 1982 Soll Analyses to Determine the Natural Background Radioactivity in SRP Surface Soils. DPST-87 260, E.I. du Pont de Nemours and Company, Savannah River Laboratory, Aiken, SC (1987).

19. WSRC, Savannah River Site Environmental Repert for 1989. WSRC-IM-90-60, Westinghouse Savannah River Company for the U.S. Department of Energy, Aiken, SC (1989).

The following documents were reviewed as part of the Unit Literature Revlew, but not cited.

* Bledsoe, H.W., SRP Baseline Hydrogeologic Investigation-Prase ll. DPST-86674, E.I. du Pont de Nemours \& Co., Savannah River Laboratory, Aiken, SC (1987)

* Christensen, E.I. and D.E. Gordon, Technical Summaxy of Groundwater Quality Protection Program at Savannah River Plant. Vol. 1: Site Geohydrology and Solid and Hazardous Wastes. DPST-83-829, E.I. du Pont de Nemours and Company, Savannah River Laboratory, Aiken, SC (1983).

- du Pont, Iechnical Summany Ground Water Quality Impact of Waste Disposal at Savannah River Plant an update of DPST 83-829* E. I. du Pont de Nemours and Company, Savannah River Plant, Aiken, SC (1987). 
- Gordon, D.E., W.E. Johnson, D.S. Kaback, B.B. Looney, R.L. Nichols, C.B. Shedrow, Characterization Recommendations for Waste Sltes at the Savannah Biver Plant. DPST-87-667, E.I. du Pont de Nemours and Company, Savannah River Laboratory, Aiken, SC (1987).

* King, C.M., W.L. Marter, B.B. Looney, and J.B. Pickett, Methodology and Parameters for Assessing Human Health Effects for Waste Sites at the Savannah Biver Plant. DPST-86-298, E.I. du Pont de Nemours and Company, Savannah River Laboratory, Aiken, SC (1987).

- Looney, B.B., J.B. Pickett, C.M. King, W.G. Holmes, W.E. Johnson, and J.A. Smith, Selection of Chemical Constituents and Estimation of Inventories for Environmental Analysis of Savannah River Plant Waste Sites. DPST-86-291, (1987).

* SAIC, Ecologic Assessment of Closure Options for Savannah River Plant Waste Sites. CORR-870116 Report to E.I. du Pont by Science Applications International Corporation, Oak Ridge, TN (1987).

- Schalles, J.F., R.R. Sharitz, J.W. Gibbons, G.J. Leversee, and J.N. Knox, Carolina Bays of the Savannah River Plant. SRO-NERP-18, National Environmental Research Park Program, Savannah River Plant, Aiken, SC (1989).

- Shields, J.D., N.D. Woody, A.S. Dicks, G.J. Hollod, J. Shalles, and G.J. Leversee, Lecations and Areas of Ponds and Carolina Bays at the Savannah Biver Plant. DP-1525, E.I. du Pont de Nemours and Company, Savannah River Plant, Aiken, SC (1982).

- USEPA, Test Methods for Exaluating Solid Waste. Volume 1. Methods for Analytes and Properties. Third Edition. EPA SW-846, Office of Solid Waste and Emergency Response, Washington, DC (1986).

- USEPA, Data Quality Objectives for Remedial Response Activities. EPA 540/G87/003, Washington, DC (1987).

* USEPA, Guidance for Conducting Remedial Investigations and Feasibility Studies Under CERCLA. Interim Final. EPA/540/G-89/004 (1988);

* WSRC, 50. Radiological Controls Manual, DPSOP 40, adapted by WSRC from E.I. du Pont de Nemours ànd Company, Savannah River Site, Aiken, SC (current version).

- WSRC, Health Protection Department Radiation Survey Procedures. DPSOL 193, adapted by WSRC from E.I. du Pont de Nemours and Company, Savannah River Site, Aiken, SC (current version). 
APPENDIX A

ANALYTES NOT FOUND

AT THE

F-AREA RAILROAD CROSSTIE PILE 
Appendix $A$

FAX COVER SHEET

Date: $8 / 22 / 91$

TO:

Name: Chuck Travis

Address: Shrine Environmental

City: Aiken

State: $5 C$

Regular Phone No: $\quad 803-649-5175$

FAX No. $803-649-5178$

Note: Here is some material you can
work with. I will call you on Monday

From:

William Far

EXPLORATION RESOURCES, INC.

425 NORTH LUMPKIN ST.

ATHENS, GA 30601

REGULAR PHONE NO.: (404) 353-7983

FAX NO.: (404) 546-8111

Na. of poses including this cover sheet 6 


\section{MEMO}

TO: William Fay

FROM: Kathleen Gore $K_{q}$

DATE: August 23, 1991

SUBJECT: Tieyard core analyses

Each of the four cores, FRT-1HP, FRT-3HP, FRT-4HP, and FRT-5HP, had 7 sediment samples. The analyses requested for each core were:

$\mathrm{pH}$, specific conductance, TOC samples $1-7$ (except sample 4 of FRT-4HP)

App. IX Inorganics:

Cyanide, sulfide

App. IX metals

samples 1.2

samples 1.7

App. IX Organics

VOA

samples 1.7

BNA

Pest/Herb

Dioxins/Furans

samples 1.7

samples 1.2

samples 1.2

samples 1.7

Rads

Gross alpha

Nonvolatile beta

Total radium

Gamma PHA (only Th-232 for FRT-1 and -5; Cs-137 and Th-232 for FRT-3 and -4.)

The following analytes from the Appendix IX Inorganics suite, Metals narratives, were not detected in any samples:

Antimony

Arsenic

Barium

Beryllium

Cadmium

Cobalt
Nickel

Selenium

Silver

Thallium

Tin 
The following analytes from the Appendix IX Organics suite, Dloxin/Furan subsuite, Dioxin narrative, were not detected in any samples:

$\mathrm{PeCDD}$

$\mathrm{PeCDF}$

Hexachlorodibenzo-p-dioxin isomers

Hexachlorodibenzo-p-furan isomers

Tetrachlorodibenzo-p-dioxin isomers

Tetrachlorodibenzo-p.furan isomers

The following analytes from the Appendix IX Organics suite, Herb/Pest subsuite, Organophosphorus pesticide narrative, were not detected in any samples:

Diazinon

Dimethoate

Disulfoton

Famphur

Ethyl parathion
Methy! parathion

Phorace

Sulfotepp

Thionazin

0,0,0-Triethyl phosphorothioate

The following analytes from the Appendix IX Organics suite, Herb/Pest subsuite, Pest/PCB narrative, were not detected in any samples:

$\begin{array}{ll}\text { Aldrin } & \text { Endrin aldehyde } \\ \text { alpha-Benzene hexachloride } & \text { Heptachlor } \\ \text { delta-Benzene hexachloride } & \text { Heptachlor epoxide } \\ \text { gamma-Benzene hexachloride (Lindane) } & \text { Isodrin } \\ \text { alpha-Chlordane } & \text { Kepone } \\ \text { gamma-Chlordane } & \text { Methoxychlor } \\ 4,4^{\prime} \cdot \text { DDD } & \text { Aroclor-1016 } \\ 4,4^{\prime} \cdot \text { DDE } & \text { Aroclor-1221 } \\ 4,4^{\prime} \cdot \text { DDT } & \text { Aroclor }-1232 \\ \text { Dieldrin } & \text { Aroclor }-1242 \\ \text { Endosulfan I } & \text { Aroclor-1248 } \\ \text { Endosulfan II } & \text { Aroclor-1254 } \\ \text { Endosulfan sulfate } & \text { Aroclor-1260 } \\ \text { Endrin } & \text { Toxaphene }\end{array}$

The following analytes from the Appendix IX Organics suite, GC/MS Volatile narrative, were not detected in any samples:

2,4-Dichlorophenoxyacetic acid

2,4,5-TP (Silvex)

2,4,5-Trichlorophenoxyacetic acid 
The following analytes from the Appendix IX Organics suite, GC/MS Volatile narrative, were not detected in any samples:

Acetonitrile
Acrolein
Acrylonitrile
Benzene
Bromodichloromethane
Bromoform
Bromomethane (Methyl bromide)
2-Butanone
Carbon disulfide
Carbon tetrachloride
Chlorobenzene
2-Chloro-1,3-butadiene
Chloroethane
Chloroethene (Vinyl chloride)
Chloroform
Chloromethane (Methyl chloride)
3-Chloropropene
Dibromochloromethane
1,2-Dibromo-3-chloropropane
1,2-Dibromoethane
Dibromomethane
trans-1,4-Dichloro-2-butene
1,1-Dichloroethane
1,2-Dichloroethane

The following analytes from the Appendix IX Organics suite, Semivolatiles narrative, were not detected in any samples:

Acenaphthene

Acetophenone

Acenaphthylene

2-Acetylaminofluorene

4-Aminobipheny!

Aniline

Anthracene

Aramite

Benzo[a]anthracene

Benzo[2]pyrene

Benzoic acid

Benzo[b]fluoranthene

Benzo[ $g, h, i]$ perylene

Benzo[k]fluoranthene

\author{
1,1.Dichloroethylene \\ Dichlorodifluoromethase \\ Dichloromethane (Methylene chloride) \\ 1,2-Dichloropropane \\ cisn-1,3-Dichloropropene \\ trans-1,3-Dichloropropene \\ Ethylbenzene \\ 2.Hexanone \\ Iodomethane (Methyl iodide) \\ Isobutyl alcohol \\ Methacrylonitrile \\ 4-Methyl-2-pentanone \\ Propionitrile \\ Styrene \\ 1,1,1,2-Tetrachloroethane \\ 1,1,2,2-Tetrachloroethane \\ Tetrachloroethylene \\ 1,1,1-Trichloroethane \\ 1,1,2. Trichloroethane \\ Trichloroethylene \\ Trichlorofluoromethane \\ 1,2,3-Trichloropropane \\ Vinyl acetate \\ Xylenes
}

Benzyl alcohol

Bis(2-chloroethyl) ether

Bis(2-chloroethoxy)methane

Bis(2-chloroisopropyl) ether

Bis(2-ethylhexyl) phthalate

4-Bromophenyl phenyl ether

Butylbenzyl phthalate

2.sec-Butyl-4,6-dinitrophenol

4.Chloroaniline

Chlorobenzilate

2.Chloronaphthalene

2-Chlorophenol

4.Chlorophenyl phenyl ether

Chrysene 
m.Cresol (3-Methylphenol)

o.Cresol (2.Methylphenol)

p-Cresol (4-Methylphenol)

Diallate

Dibenz[a,h]anthracene

Dibenzofuran

Di-a-butyl phthalate

1,2.Dichlorobenzene

1,3-Dichlorobenzene

1,4-Dichlorobenzene

3,3' -Dichlorobenzidine

2,4-Dichlorophenol

2,6.Dichlorophenol

Diethy! phthalate

p-Dimethylaminozzobenzene

7,12. Dimethylbenz[a]anthracene

3,3 $\int$.Dimethylbenzidine

a,2-Dimethylphenethylamine

2,4-Dimethyl phenol

Dimethyl phthalate

1,3-Dinitrobenzene

2,4-Dinitrophenol

2,4-Dinitrotoluene

2,6-Dinitrotoluene

Di-n-octyl phthalate

1,4-Dioxane

Diphenylamine

Ethyl methacrylate

Ethy! methanesulfonate

Fluoranthene

Fluorene

Hexachlorobenzene

Hexachlorobutadiene

Hexachlorocyclopentadiene

Hexachloroethane

Hexachlorophene

Hexachloropropene

Indeno $[1,2,3-c, d]$ pyrene

Isophorone

Isosafrole

Methapyrilene

3.Methylcholanthrene

3-Methyl-4-chlorophenol

2-Methyl-4,6-Dinitrophenol

Methyl methacrylate

Methyl methanesulfonate
2.Methylnaphthalene

Naphthalene

1,4-Naphthoquinone

1.Naphthylamine

2.Naphthylamine

2-Nitroaniline

3-Nitroaniline

4.Nitroaniline

Nitrobenzene

2.Nitrophenol

4.Nitrophenol

4-Nitroquinoline-1-oxide

N-Nitrosodi-n-butylamine

N.Nitrosodiethylamine

N.Nitrosodimethylamine

N.Nitrosodiphenylamine

N.Nitrosodi-n-propylamine

N.Nitrosomethylethylamine

N.Nitrosomorpholine

N.Nitrosopiperidine

N-Nitrosopyrrolidine

5-Nitro-0-toluidine

Pentachlorobenzene

Pentachloroethane

Pentachloronitrobenzene

Pentachlorophenol

Phenacetin

Phenanthrene

Phenols

p-Phenylenediamine

2-Picoline

Pronamide

Pyrene

Safrole

1,2,4,5-Tetrachlorobenzene

$2,3,4,6$-Tetrachlorophenol

o-Toluidine

1,2,4-Trichlorobenzene

2,4,5-Trichlorophenol

2,4,6-Trichlorophenol

1,3,5-Trinitrobenzene 
Analytes detected only with modifier (l.e., otherwise not detected at all):

beta-Benzene hexachloride (modifier B) (Pesticide/PCB narrative of Pest/Herb subsulte of App, IX Organics suite)

Dichloromethane (Methylene chloride) (modifier B) (GC/MS Volatiles narrative of App. IX Organic suite)

Cesium-137 (result: $0.2 \pm 0.2$ ) (Rads)

The following are the analytes that had results above detection limits.

Analytes detected:

pH, spec. cond., TOC

Acetone

Chromium

Copper

Cyanide

Lead

Mercury

Pyridine

Sulfide

TOC

Toluene

Vanadium

Zinc

Rads
Suite:

$\mathrm{pH}$, spec. cond, TOC, Inorganics aarrative App. IX VOA; GC/MS Vol, narrative

App. IX Inorganics; Metals narrative

App. IX Inorganics; Metals narrative

App. IX Inorganics; Inorganics narrative

App. IX Inorganics; Metals narrative

App. IX Inorganics; Metals narrative

App. IX BNA; Semivolatile narrative

App. IX Inorganics; Inorganics narrative

App. IX Inorganics; Inorganics narrative

App. IX VOA; GC/MS Volatiles aarrative

App. IX Inorganics; Metals narrative

App. IX Inorganics; Metals narrative

Rads from subcontracting laboratory (Eberline)

I wasn't able to find results for analyses of 1,2-Dichloroethene. 
APPENDIX B

GROUNDWATER MONITORING DATA

FBP WELLS

Third Quarter 1984 to First Quarter 1991 
DATA RETRIEVED 7/2/91

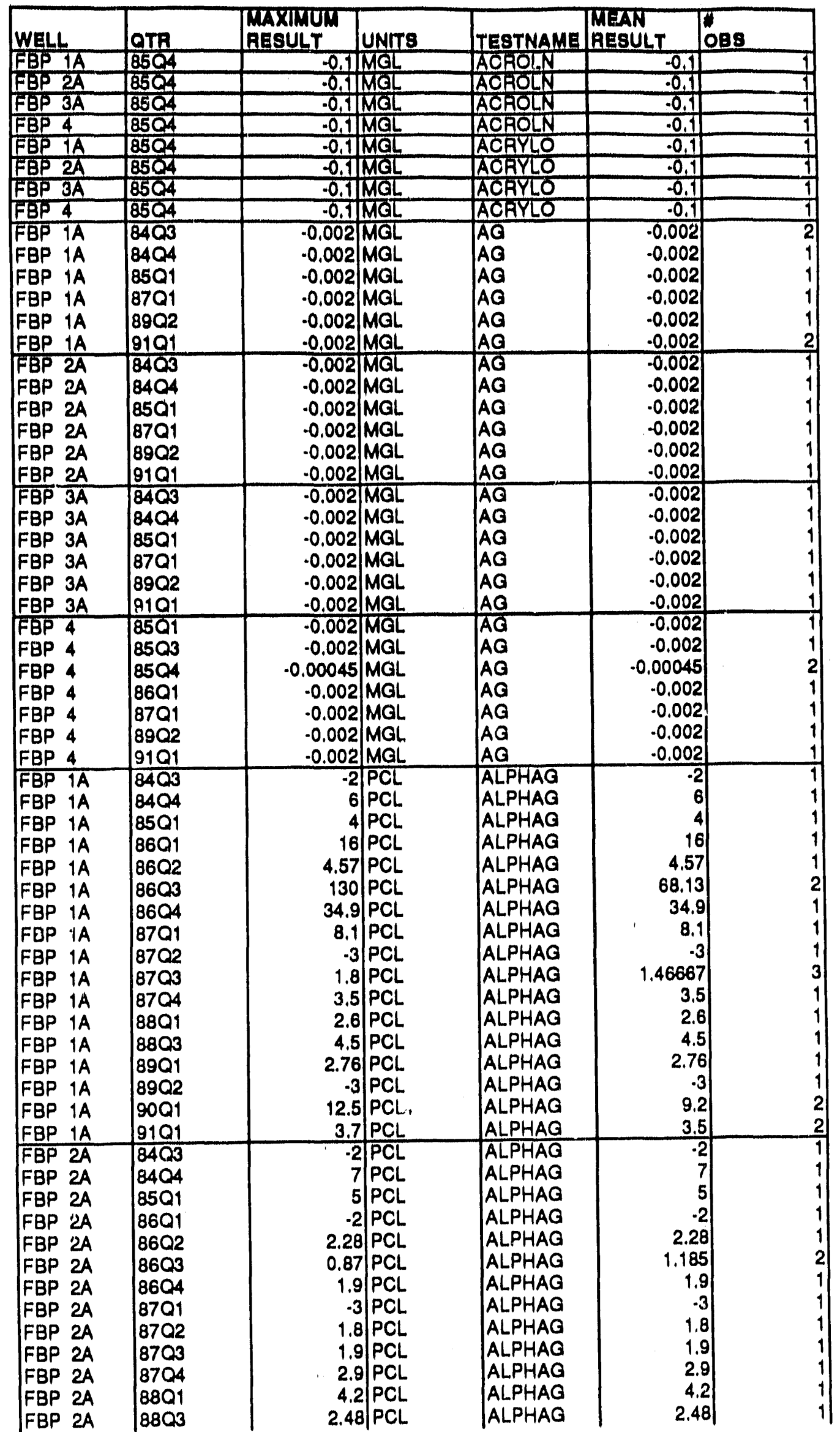

Plot Fllu of Laboratory Analyses at FBP Monitoring Wolls - Page 1 
DATA RETRIEVED 7/2/91

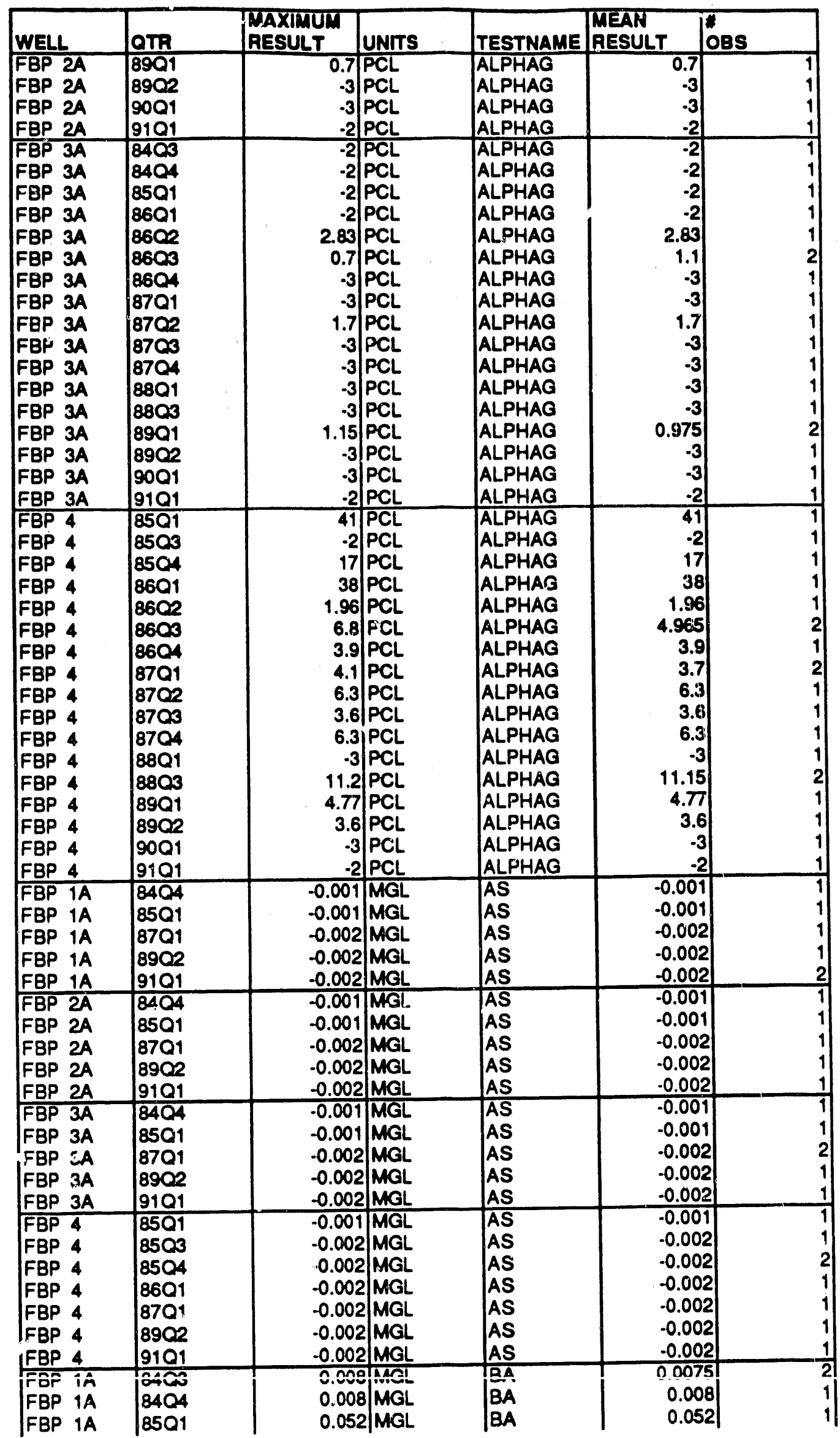

Plot File of Laboratory Analyses at FBP Monitoring Wells - Page 2 
DATA RETRIEVED 7/2/91

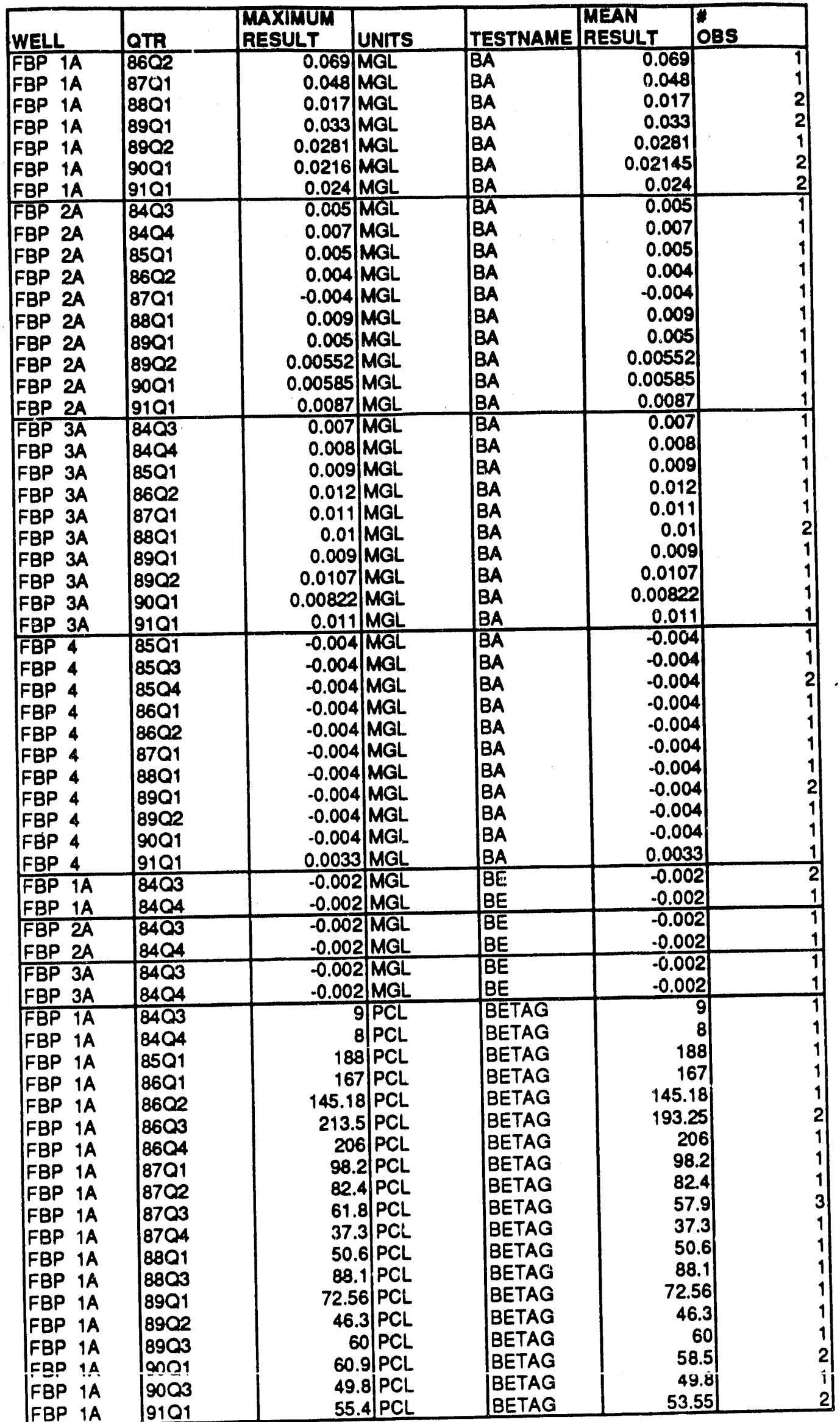

Plot File of Laboratory Analyses at FBP Monitoring Wells - Page 3 
DATA RETRIEVED 7/2/91

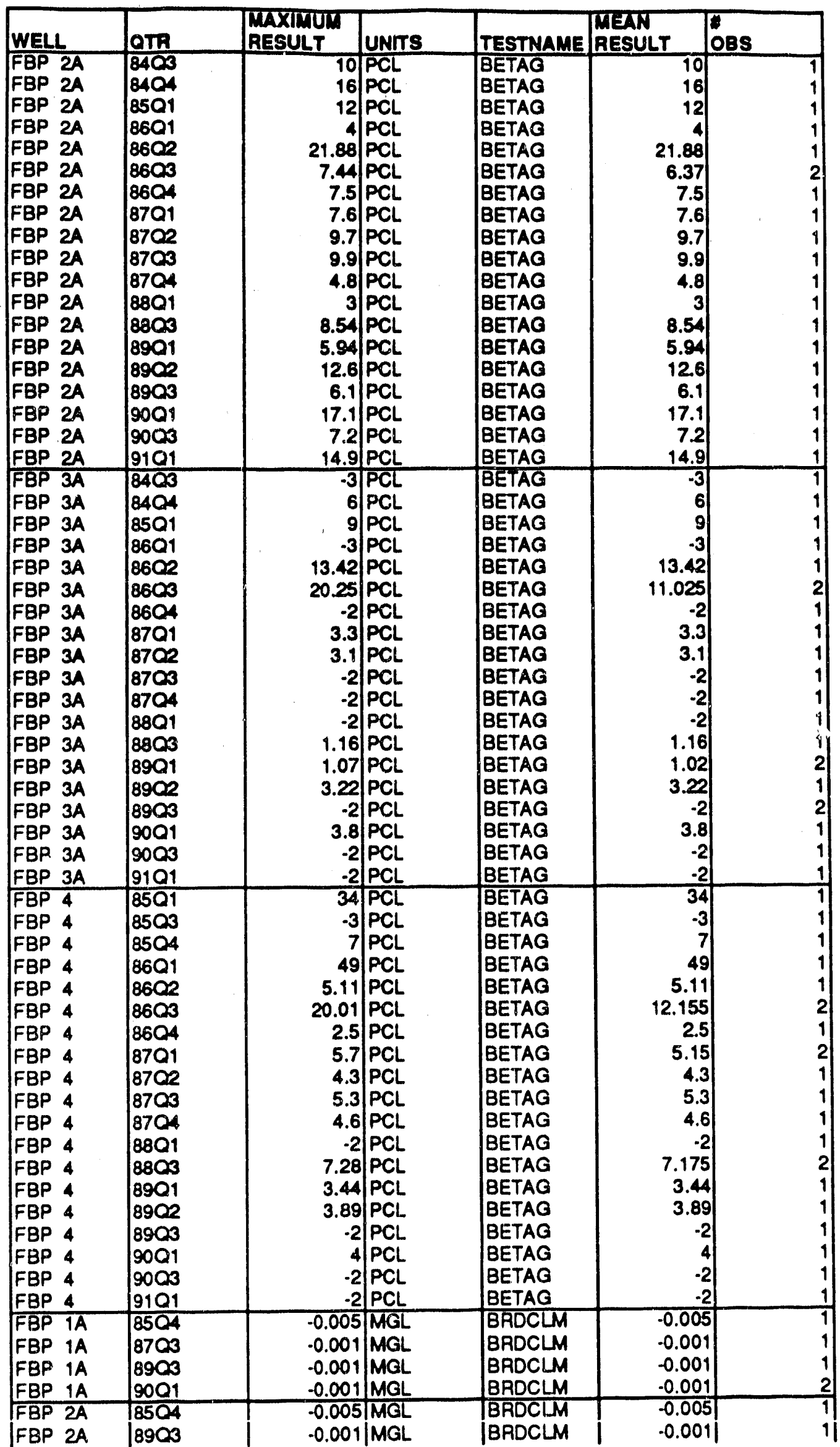

Plot File of Laboratory Analyses at FBP Monitoring Wolls - Page 4 
DATA RETRIEVED 7/2/91

\begin{tabular}{|c|c|c|c|c|c|c|}
\hline WELL & aTR & $\begin{array}{l}\text { MAXINUT } \\
\text { RESULT }\end{array}$ & UNITS & TESTNAME & $\begin{array}{l}\text { MEAN } \\
\text { RESULT }\end{array}$ & OBS \\
\hline FBP 2A & 9001 & 0.001 & MGL & BRDCLM & -0.001 & 1 \\
\hline FBP 3A & 8504 & -0.005 & MGL & BADCLM & .0 .005 & 1 \\
\hline $\begin{array}{ll}F B P & 3 A \\
\text { FBP } & 3 A\end{array}$ & $\begin{array}{l}8903 \\
9001\end{array}$ & $\begin{array}{l}-0.001 \\
-0.001\end{array}$ & MGL & BRDCLM & $\begin{array}{l}-0.001 \\
-0.001\end{array}$ & $\begin{array}{l}2 \\
1\end{array}$ \\
\hline FBP 4 & 8504 & -0.005 & $\frac{M G L}{M G L}$ & BRDCLM & $\frac{-0.0011}{-0.005}$ & 1 \\
\hline FBP 4 & 8903 & -0.001 & MGL & BRDCLM & -0.001 & 1 \\
\hline FBP 4 & 9001 & -0.001 & $M G L$ & BRDCLM & -0.001 & 1 \\
\hline FBP IA & 8701 & 13.9 & MGL & CA & 13.9 & 1 \\
\hline FBP $1 A$ & 8801 & 6.12 & MGL & CA & 6.09 & 2 \\
\hline FBP $1 A$ & 8901 & 10.8 & MGL & CA & 9.7 & 2 \\
\hline FBP $1 A$ & 8902 & 8.02 & MGL & CA & 8.02 & 1 \\
\hline FBP $1 A$ & 9001 & 6.75 & MGL & CA & 6.605 & 2 \\
\hline FBP 1A & 9101 & 7 & MGL & $\mathrm{CA}$ & 6.95 & 2 \\
\hline FBP 2A & $87 Q 1$ & 1.23 & MGL & $\mathrm{CA}$ & 1.23 & 1 \\
\hline FBP 2A & 8801 & 2.5 & MGL & CA & 2.5 & 1 \\
\hline FBP 2A & 8901 & 2.79 & MGL & CA & 2.79 & 1 \\
\hline FBP 2A & 8902 & 1.85 & MGL & CA & 1.85 & 1 \\
\hline FBP 2A & 9001 & 2.2 & MGL & CA & 2.2 & 1 \\
\hline FBP 2A & 9101 & 2.3 & MGL & CA & 2.3 & $\frac{1}{4}$ \\
\hline FBP $3 A$ & 8701 & 4.1 & MGL & CA & 4.1 & 1 \\
\hline FBP $3 A$ & 88Q1 & 3.7 & MGL & CA & 3.585 & 2 \\
\hline FBP $3 A$ & 8901 & 3.9 & MGL & CA & 3.9 & 1 \\
\hline FBP $3 A$ & 8902 & 4.05 & MGL & CA & 4.05 & 1 \\
\hline FBP $3 A$ & 9001 & 3.53 & MGL & CA & 3.53 & 1 \\
\hline FBP $3 A$ & 9101 & 3.3 & MGL & CA & 3.3 & 1 \\
\hline FBP 4 & 8701 & 0.434 & MGL & $\overline{C A}$ & 0.434 & 1 \\
\hline FBP 4 & 8801 & 0.584 & MGL & CA & 0.584 & 1 \\
\hline FBP 4 & 8901 & 0.572 & MGL & CA & 0.53 & 2 \\
\hline FBP 4 & 8902 & 0.58 & MGL & CA & 0.58 & 1 \\
\hline FBP 4 & 9001 & 0.339 & MGL & CA & 0.339 & 1 \\
\hline FBP 4 & 9101 & 0.34 & MGL & CA & 0.34 & 1 \\
\hline FBP 1A & 8703 & -0.003 & MGL & CCL2F2 & -0.003 & 1 \\
\hline FBP $1 A$ & 8504 & -0.005 & MGL & CCL3F & -0.005 & 1 \\
\hline FBP $1 A$ & 8703 & 0.00833 & MGL & CCL3F & 0.00833 & 1 \\
\hline FBP $1 A$ & 8903 & 0.008 & MGL & CCL3F & 0.008 & 1 \\
\hline FBP $1 A$ & 9001 & .0 .001 & MGL & CCL3F & -0.001 & 2 \\
\hline FBP. 2A & 8504 & .0 .005 & MGL & CCL3F & -0.005 & 1 \\
\hline FBP $2 A$ & 8903 & 0.015 & MGL & CCL3F & 0.015 & 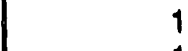 \\
\hline FBP $2 A$ & 9001 & 0.007 & $M G L$ & CCL3F & 0.007 & 1 \\
\hline FBP $3 A$ & 8504 & -0.005 & MGL & CCL3F & -0.005 & $\overline{1}$ \\
\hline FBP $3 A$ & 8903 & 0.006 & MGL & CCL3F & 0.0055 & \\
\hline FBP $3 A$ & 9001 & 0.001 & MGL & CCL $3 F$ & 0.001 & 1 \\
\hline FBP 4 & 8504 & -0.005 & MGL & CCL3F & -0.005 & 7 \\
\hline FBP 4 & 8903 & -0.001 & MGL & CCL3F & -0.001 & \\
\hline FBP 4 & 9001 & -0.001 & $M G L$ & CCL.3F & -0.001 & 1 \\
\hline FBP $1 \mathrm{~A}$ & 8504 & -0.005 & MGL & CCL4 & -0.005 & $\overline{1}$ \\
\hline FBP $1 A$ & 8603 & -0.001 & MGL & CCL.4 & -0.001 & 2 \\
\hline FBP $1 A$ & 8701 & -0.001 & MGL & CL4 & -0.001 & 1 \\
\hline FBP $1 A$ & 8702 & -0.001 & MGL & LL4 & -0.001 & 1 \\
\hline FBP $1 A$ & 8703 & -0.001 & MGL & CCL4 & -0.001 & 3 \\
\hline FBP $1 A$ & 8704 & -0.001 & MGL & CCL4 & $\cdot 0.001$ & 1 \\
\hline FBP $1 A$ & 88Q1 & -0.001 & MGL & CCL4 & -0.001 & 1 \\
\hline FBP $1 A$ & 8803 & -0.001 & MGL & CL4 & -0.001 & 2 \\
\hline FBP $1 A$ & 8901 & -0.001 & MGL & LL4 & -0.001 & 1 \\
\hline FBP $1 A$ & 89023 & -0.001 & MGL & 44 & -0.001 & 1 \\
\hline FBP $1 A$ & 9001 & -0.001 & MGL & L4 & -0.001 & 2 \\
\hline FBP $1 A$ & 9003 & -0.001 & MGL & L4 & -0.001 & 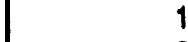 \\
\hline FBP $1 \mathrm{~A}$ & 9101 & -0.001 & MGL & LL4 & -0.001 & 2 \\
\hline FBP 2A & 8504 & 0.017 & MGL & CCL4 & 0.017 & $\bar{T}$ \\
\hline FBP 2A & 8603 & 0.00734 & MGL & CCL4 & 0.0072 & 2 \\
\hline FBP 2A & 8604 & 0.00462 & MGL & CCL4 & 0.00462 & \\
\hline FBP $2 A$ & 8701 & $\begin{array}{r}0.002 \\
0.00503\end{array}$ & MGL & CCL4 & 0.002 & \\
\hline 2 & 12702 & 0.0059? & IMSEI & 4 & 0.00593 & \\
\hline
\end{tabular}

Plot File of Laboratory Analyses at FBP Monitoring Wells - Page 5 
DATA RETRIEVED 7/2/91

\begin{tabular}{|c|c|c|c|c|c|c|}
\hline WELL & aTR & $\begin{array}{l}\text { MAXIMUM } \\
\text { RESULT }\end{array}$ & UNITS & TESTNAME & $\begin{array}{l}\text { MEAN } \\
\text { RESULT }\end{array}$ & OBS \\
\hline $\begin{array}{ll}\text { FBP } & 2 A \\
\text { FBP } & 2 A \\
\text { FBP } & 2 A \\
\text { FBP } & 2 A \\
\text { FBP } & 2 A \\
\text { FBP } & 2 A \\
\text { FBP } & 2 A \\
\text { FBP } & 2 A \\
\text { FBP } & 2 A\end{array}$ & $\begin{array}{l}87 Q 3 \\
8704 \\
8801 \\
8803 \\
8901 \\
8903 \\
9001 \\
9003 \\
9101\end{array}$ & $\begin{array}{r}0.001 \\
0.0148 \\
0.0124 \\
0.008 \\
0.00673 \\
-0.001 \\
0.007 \\
0.008 \\
0.004\end{array}$ & $\begin{array}{l}\text { MGL } \\
M G L \\
M G L \\
M G L \\
M G L \\
M G L \\
M G L \\
M G L \\
\text { MGL }\end{array}$ & $\begin{array}{l}\mathrm{CCL4} \\
\mathrm{CCL} 4 \\
\mathrm{CCL} 4 \\
\mathrm{CCL} 4 \\
\mathrm{CCL4} \\
\mathrm{CCL} 4 \\
\mathrm{CCL} 4 \\
\mathrm{CCL} 4 \\
\mathrm{CCL} 4\end{array}$ & $\begin{array}{r}0.001 \\
0.0148 \\
0.0124 \\
0.008 \\
0.00673 \\
-0.001 \\
0.007 \\
0.008 \\
0.004\end{array}$ & $\begin{array}{l}1 \\
1 \\
1 \\
1 \\
1 \\
1 \\
1 \\
1 \\
1 \\
\end{array}$ \\
\hline $\begin{array}{l}\text { FBP } 3 A \\
\text { FRP }\end{array}$ & 8504 & $\begin{array}{r}-0.005 \\
0\end{array}$ & MGL & CCL4 & $\begin{array}{r}-0.005 \\
0\end{array}$ & $\begin{array}{l}1 \\
1\end{array}$ \\
\hline $\begin{array}{l}\text { FBP } 3 A \\
\text { FBP } 3 A\end{array}$ & $\begin{array}{l}8603 \\
87 Q 1\end{array}$ & $\begin{array}{r}0.00119 \\
-0.001\end{array}$ & $\begin{array}{l}\text { MGL } \\
\text { MGL }\end{array}$ & CCL4 4 & $\begin{array}{r}0.00119 \\
-0.001\end{array}$ & $\begin{array}{l}1 \\
1\end{array}$ \\
\hline $\begin{array}{l}\text { FBP } 3 A \\
\text { FBP }\end{array}$ & $\begin{array}{l}87 Q 2 \\
8703\end{array}$ & $\begin{array}{l}-0.001 \\
-0.001\end{array} \mid$ & $\begin{array}{l}\text { MGL } \\
\text { MGL }\end{array}$ & $\begin{array}{l}\mathrm{CCL} 4 \\
\mathrm{CCL} 4\end{array}$ & $\begin{array}{l}-0.001 \\
-0.001\end{array}$ & $\begin{array}{l}1 \\
1\end{array}$ \\
\hline $\begin{array}{l}\text { FBP } 3 A \\
\text { FAP }\end{array}$ & 8704 & -0.001 & MGL & CCL4 & -0.001 & 1 \\
\hline FBP 3A & 8801 & $\begin{array}{l}-0.001 \\
-0.001\end{array}$ & MGL & CCL4 & $\begin{array}{l}-0.001 \\
-0.001\end{array}$ & $\begin{array}{l}1 \\
1\end{array}$ \\
\hline FBP 3A & 8901 & -0.001 & MGL & CCL4 & -0.001 & 1 \\
\hline $\begin{array}{l}\text { FBP } 3 A \\
\text { FBP } 3 A\end{array}$ & $\begin{array}{l}8903 \\
9001\end{array}$ & $\begin{array}{l}-0.001 \\
-0.001\end{array} \mid$ & $\begin{array}{l}\text { MGL } \\
\text { MGL }\end{array}$ & $\begin{array}{l}\text { CCL4 } \\
\text { CCL4 }\end{array}$ & $\begin{array}{l}-0.001 \\
-0.001\end{array}$ & $\begin{array}{l}2 \\
1\end{array}$ \\
\hline FBP $3 A$ & 9003 & -0.001 & MGL & CCL4 & -0.001 & 1 \\
\hline FBP $3 A$ & 9101 & -0.001 & MGL & $\mathrm{CCL}_{4}$ & -0.001 & 1 \\
\hline FBP 4 & 8504 & -0.005 & MGL & CCL4 & -0.005 & 1 \\
\hline FBP 4 & 8603 & -0.001 & MGL & CCL4 & -0.001 & 1) \\
\hline FBP 4 & 8701 & -0.001 & MGL & CCL4 & -0.001 & 1 \\
\hline FBP 4 & 8702 & -0.001 & MGL & $\mathrm{CCL} 4$ & -0.001 & 1 \\
\hline $\begin{array}{l}\text { FBP } 4 \\
\text { FBP } 4\end{array}$ & $\begin{array}{l}8703 \\
8704\end{array}$ & $\begin{array}{l}-0.001 \\
-0.001\end{array}$ & $\begin{array}{l}\text { MGL } \\
\text { MGL }\end{array}$ & $\begin{array}{l}\mathrm{CCL} 4 \\
\mathrm{CCL} 4\end{array}$ & $\begin{array}{l}-0.001 \\
-0.001\end{array}$ & 2 \\
\hline FBP 4 & 8801 & -0.001 & MGL & CCLA & -0.001 & 2 \\
\hline FBP 4 & $\begin{array}{l}8803 \\
8001\end{array}$ & -0.001 & MGL & $\begin{array}{l}\text { CCL4 } \\
\text { CCL4 }\end{array}$ & $\begin{array}{l}-0.001 \\
-0.001\end{array}$ & $\begin{array}{l}1 \\
1\end{array}$ \\
\hline $\begin{array}{ll}\text { FBP } & 4 \\
\text { FBP } & 4\end{array}$ & $\begin{array}{l}8901 \\
8903\end{array}$ & $\begin{array}{l}-0.001 \\
-0.001\end{array}$ & $\begin{array}{l}\text { MGL } \\
\text { MGL }\end{array}$ & CCL44 & -0.001 & 1 \\
\hline FBP 4 & 9001 & -0.001 & MGL & CCL4 & -0.001 & 1 \\
\hline FBP 4 & 9003 & -0.001 & MGL & CCL4 & -0.001 & 1 \\
\hline FBP 4 & 9101 & -0.001 & MGL & CCL 4 & -0.001 & 1 \\
\hline FBP $1 A$ & 8403 & -0.002 & MGL & $C D$ & -0.002 & 2 \\
\hline FBP $1 A$ & 8404 & -0.002 & MGL & $C D$ & -0.002 & 1 \\
\hline FBP $1 A$ & 8501 & -0.002 & MGL & $C D$ & -0.002 & 1 \\
\hline FBP 1A & $86 Q 1$ & -0.001 & MGL & $C D$ & -0.001 & 1 \\
\hline FBP $1 A$ & $86 Q 3$ & -0.002 & MGL & $\begin{array}{l}C D \\
C D\end{array}$ & $\begin{array}{l}-0.002 \\
-0.002\end{array}$ & $\begin{array}{l}1 \\
1\end{array}$ \\
\hline $\begin{array}{ll}\text { FBP } & 1 A \\
\text { FBP } & 1 A\end{array}$ & $\begin{array}{l}8701 \\
8702\end{array}$ & $\begin{array}{l}-0.002 \\
-0.002\end{array}$ & $\begin{array}{l}\text { MGL } \\
\text { MGL }\end{array}$ & & $\begin{array}{l}-0.002 \\
-0.002\end{array}$ & $\begin{array}{l}1 \\
1\end{array}$ \\
\hline FBP IA & 8703 & -0.002 & MGL & & -0.003 & 4 \\
\hline FBP $1 A$ & 8704 & -0.002 & MGL & & -0.002 & 1 \\
\hline FBP $1 A$ & 8801 & -0.002 & MGL & & -0.002 & 2 \\
\hline $\begin{array}{ll}\text { FBP } & 1 A \\
\text { FBP } & 1 A\end{array}$ & $\begin{array}{l}8902 \\
9101\end{array}$ & $\begin{array}{r}-0.002 \\
-0.002\end{array}$ & $\begin{array}{l}\text { MGL } \\
\text { MGL }\end{array}$ & & $\begin{array}{l}-0.002 \\
-0.002\end{array}$ & $\begin{array}{l}1 \\
2\end{array}$ \\
\hline FBP $2 A$ & 8403 & -0.002 & MGL & $C D$ & -0.002 & 7 \\
\hline FBP $2 A$ & 8404 & -0.002 & MGL & $C D$ & -0.002 & 1 \\
\hline FBP 2A & $85 Q 1$ & -0.002 & MGL & CD & -0.002 & 1 \\
\hline FBP 2A & 8601 & -0.001 & MGL & $C D$ & -0.001 & 1 \\
\hline FBP 2A & 8603 & -0.002 & MGL & $\begin{array}{l}C D \\
C D\end{array}$ & $\begin{array}{l}-0.002 \\
-0.002\end{array}$ & 1 \\
\hline $\begin{array}{ll}\text { FBP } & 2 A \\
\text { FRP } & 2 A\end{array}$ & $\begin{array}{l}8701 \\
8702\end{array}$ & $\begin{array}{l}-0.002 \\
-0.002\end{array}$ & $\begin{array}{l}\text { MGL } \\
\text { MGL }\end{array}$ & $\mid \begin{array}{l}C D \\
C D\end{array}$ & $\begin{array}{l}-0.002 \\
-0.002\end{array}$ & 1 \\
\hline $\mid \begin{array}{ll}F B P & 2 A \\
F B P & 2 A\end{array}$ & $\begin{array}{l}8702 \\
8703\end{array}$ & -0.002 & MGL & c & -0.002 & 1 \\
\hline FBP 2A & 8704 & -0.002 & MGL & C & -0.002 & 1 \\
\hline $2 A$ & 8801 & -0.002 & MGL & & $\begin{array}{l}-0.002 \\
-0.002\end{array}$ & 1 \\
\hline $\begin{array}{l}2 A \\
2 A\end{array}$ & $\begin{array}{l}89 Q 2 \\
9101\end{array}$ & $\begin{array}{l}-0.002 \\
-0.002\end{array}$ & $\begin{array}{l}\text { MGL } \\
\text { MGL }\end{array}$ & CD & $\begin{array}{r}-0.002 \\
-0.002 \\
\end{array}$ & 1 \\
\hline $\begin{array}{ll}F B P & 2 A \\
F B P & 3 A\end{array}$ & 8403 & $\frac{-0.00 \mathrm{c}}{0.046}$ & MGL & C & 0.046 & 4 \\
\hline FBP $3 A$ & 8404 & 0.011 & MGL & & 0.011 & \\
\hline FBP $3 A$ & 85 & -0.002 & MGL & & -0.002 & \\
\hline $3 A$ & 86 & -0.001 & |MGL & & $-0.001 \mid$ & \\
\hline
\end{tabular}

Plot Flle of Laboratory Analyses at FBP Monitoring Wells - Page 6 
DATA RETRIEVED 7/2/91

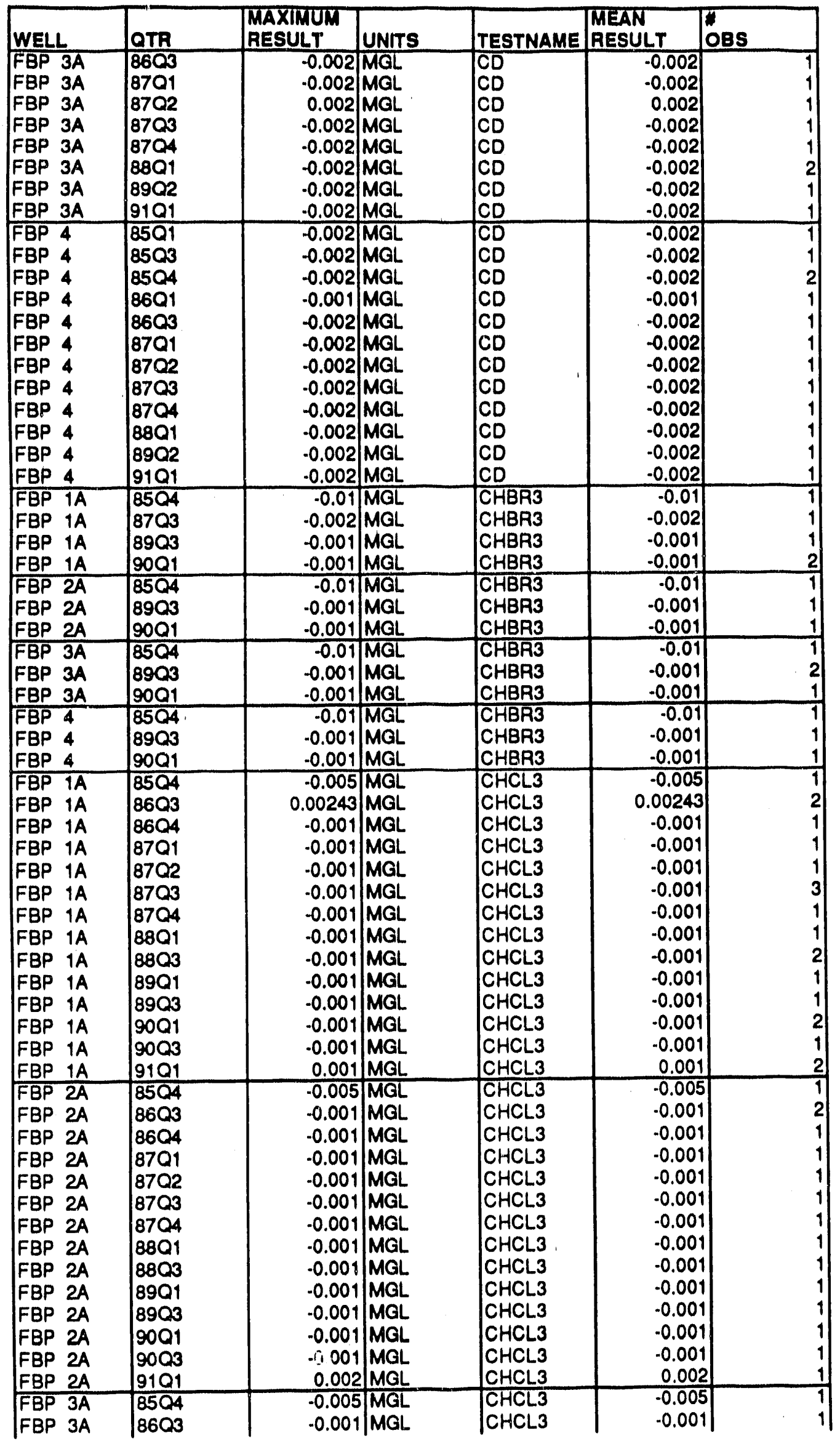

Plot File of Laboratory Analyses at FBP Monitoring Wells - Page 7 
DATA RETRIEVED 7/2/91

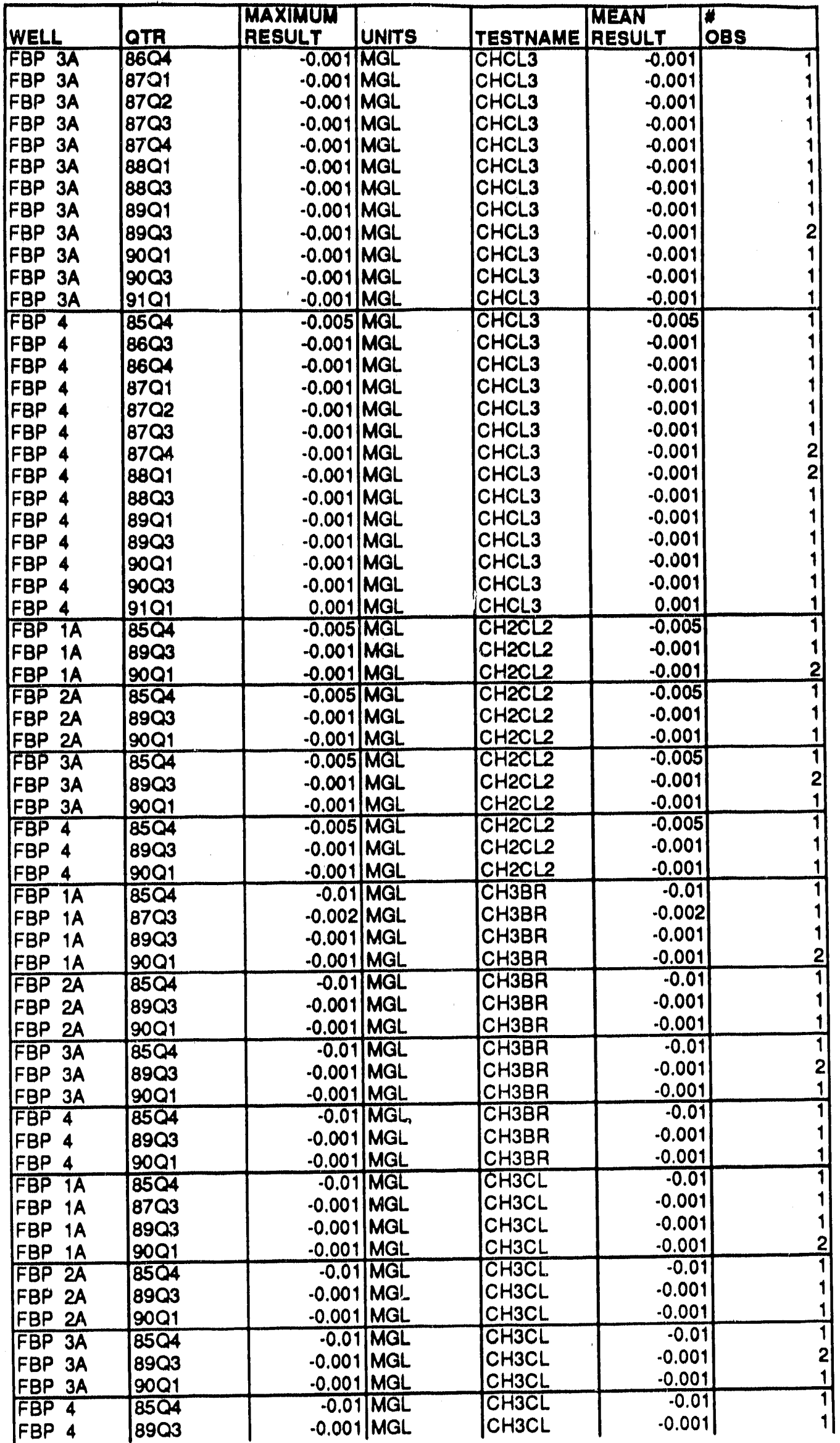

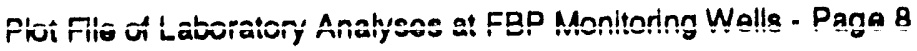


DATA RETRIEVED 7/2/91

\begin{tabular}{|c|c|c|c|c|c|c|}
\hline WELLL & atr & $\begin{array}{l}\text { MAXIMUM } \\
\text { RESULT }\end{array}$ & UNITS & TESTNAME & $\begin{array}{l}\text { MEAN } \\
\text { RESULT }\end{array}$ & OBS \\
\hline FBP 4 & 9001 & -0.001 & MGL & $\mathrm{CH} 3 \mathrm{CL}$ & .0 .001 & 1 \\
\hline FBP TA & 8403 & 2.5 & MGL & $C L$ & 2.5 & 1 \\
\hline FBP IA & 8404 & 2.5 & MGL & $C L$ & 2.5 & 1 \\
\hline FBP $1 A$ & 8501 & 2.5 & MGL & $O L$ & 2.5 & 1 \\
\hline FBP $1 A$ & $\begin{array}{l}8601 \\
8701\end{array}$ & 5.2 & MGL & $C L$ & 5.2 & 11 \\
\hline FBP $1 A$ & $\begin{array}{l}8701 \\
8902\end{array}$ & $\begin{array}{r}3.1 \\
3\end{array}$ & $\begin{array}{l}\text { MGL } \\
\text { MGL }\end{array}$ & $C L$ & 3 & 1 \\
\hline FBP $1 A$ & 9101 & 2.87 & MGL & $\mathrm{CL}$ & 2.835 & 2 \\
\hline FBP 2A & 8403 & 4.5 & MGL & $\overline{C L}$ & 4.5 & 1 \\
\hline FBP $2 A$ & 8404 & 5 & MGL & $\mathrm{CL}$ & 5 & 1 \\
\hline FBP 2A & 85Q1 & 5.5 & MGL & $C L$ & 5 & 2 \\
\hline FBP 2A & 8601 & 8.7 & MGL & $C L$ & 8.7 & 1 \\
\hline FBP 2A & 87Q1 & 3.1 & MGL & $C L$ & 3.1 & 1 \\
\hline FBP 2A & 8902 & 3.1 & MGL & $C L$ & 3.1 & 1 \\
\hline FBP $2 A$ & 9101 & 5.52 & $M G L$ & $C L$ & 5.52 & 1 \\
\hline FBP $3 A$ & 8403 & $4 \mid$ & MGL & $\mathrm{CL}$ & 4 & 1 \\
\hline FBP $3 A$ & 8404 & 4.6 & $M G L$ & $C L$ & 4.6 & 1 \\
\hline FBP $3 A$ & $85 Q 1$ & 4 & MGL & $C L$ & 4 & 1 \\
\hline FBP $3 A$ & 8601 & 6.9 & MGL & $C L$ & 6.9 & 1 \\
\hline FBP $3 A$ & 8701 & 3.5 & MGL & $C L$ & 3.5 & 1 \\
\hline FBP $3 A$ & 8902 & 3.5 & MGL & $C L$ & 3.5 & 1 \\
\hline FBP $3 A$ & 9101 & 3.62 & MGL & $\mathrm{CL}$ & 3.62 & 1 \\
\hline FBP 4 & 8501 & 2.5 & MGL & $\mathrm{CL}$ & 2.5 & 1 \\
\hline FBP 4 & 8503 & 3.27 & MGL & $\mathrm{CL}$ & 3.11 & 2 \\
\hline FBP 4 & 8504 & 2.4 & MGL & CL. & 2.4 & 1 \\
\hline FBP 4 & 8601 & 2.9 & MGL & $C L$ & 2.9 & 2 \\
\hline FBP 4 & 8701 & 3.1 & MGL & $C L$ & 3 & 2 \\
\hline FBP 4 & 8902 & 2.9 & MGL & CL & 2.9 & $\begin{array}{l}1 \\
1\end{array}$ \\
\hline FBP 4 & 9101 & 2.97 & MGL & $\mathrm{CL}$ & 2.97 & 1 \\
\hline FBP 1A & 8504 & -0.005 & MGL & CLC6H5 & -0.005 & 1 \\
\hline FBP $1 A$ & 8903 & -0.001 & MGL & CLC6H5 & -0.001 & 1 \\
\hline FBP $1 A$ & 9001 & -0.001 & MGL & CLC6H5 & -0.001 & 2 \\
\hline FBP 2A & 8504 & -0.005 & MGL & CLC6H5 & -0.005 & 1 \\
\hline FBP $2 A$ & 8903 & -0.001 & MGL & CLC6H5 & -0.001 & 1 \\
\hline FBP $2 A$ & 9001 & .0 .001 & MGL & CLC6H5 & .0 .001 & 1 \\
\hline FBP $3 A$ & 8504 & -0.005 & MGL & CLC6H5 & .0 .005 & 1 \\
\hline FBP $3 A$ & 8903 & -0.001 & MGL & CLC6H5 & -0.001 & 2 \\
\hline FBP $3 A$ & 9001 & -0.001 & MGL & CLC6H5 & -0.001 & 1 \\
\hline FBP 4 & 8504 & -0.005 & MGL & CLC6H5 & -0.005 & 1 \\
\hline FBP 4 & 8903 & -0.001 & MGL. & CLC6H5 & -0.001 & 1 \\
\hline FBP 4 & 9001 & -0.001 & $M G L$ & CLC6H5 & -0.001 & 1 \\
\hline FBP $1 A$ & 8403 & 0) & APC & COLOA & 0 & 1 \\
\hline FBP $1 A$ & 8404 & 0 & APC & COLOR & 0 & 1 \\
\hline FBP 2A & 8403 & 0 & APC & COLOR & 0 & 1 \\
\hline FBP $2 A$ & 8404 & 0 & APC & COLOR & o. & 1 \\
\hline FBP $3 A$ & 8403 & 0 & APC & COLOR & 0 & $\frac{1}{1}$ \\
\hline FBP $3 A$ & 18404 & 0 & APC & COLOA & 이 & 1 \\
\hline FBP $1 \mathrm{~A}$ & 8403 & 35.2 & UMHC & COND & 35.2 & $\frac{1}{1}$ \\
\hline FBP $1 A$ & 8404 & 25 & UMHC & COND & 25 & 1 \\
\hline FBP $1 A$ & 8501 & 192.6 & UMHC & COND & 192.6 & 1 \\
\hline FBP $1 A$ & 8502 & 160.4 & UMHC & COND & 160.4 & 1 \\
\hline FBP $1 A$ & 8503 & 204.6 & UMHC & COND & $\begin{array}{r}204.6 \\
303\end{array}$ & 1 \\
\hline FBP $1 A$ & $85 \bigcirc 4$ & $\begin{array}{l}393 \\
225\end{array}$ & UMHC & $\begin{array}{l}\text { COND } \\
\text { COND }\end{array}$ & $\begin{array}{l}393 \\
225\end{array}$ & $\begin{array}{l}2 \\
1\end{array}$ \\
\hline $\begin{array}{ll}\text { FBP } & 1 A \\
\text { FBP } & 1 A\end{array}$ & $\mid \begin{array}{l}8601 \\
8602\end{array}$ & $\begin{array}{l}225 \\
243\end{array}$ & $\begin{array}{l}\text { UMHC } \\
\text { UMHC }\end{array}$ & COND & 243 & 1 \\
\hline FBP $1 A$ & 8603 & 246 & UMHC & COND & $\begin{array}{r}244.4 \\
241\end{array}$ & 2 \\
\hline FBP $1 A$ & 8604 & $\begin{array}{l}241 \\
169\end{array}$ & $\begin{array}{l}\text { UMHC } \\
\text { UMHC }\end{array}$ & $\begin{array}{l}\text { COND } \\
\text { COND }\end{array}$ & $\begin{array}{l}241 \\
169\end{array}$ & $\begin{array}{l}1 \\
1\end{array}$ \\
\hline $\begin{array}{ll}\text { FBP } & 1 A \\
\text { FBP } & 1 A\end{array}$ & $\begin{array}{l}87 Q 1 \\
87 Q 2\end{array}$ & $\begin{array}{l}169 \\
117\end{array}$ & $\begin{array}{l}\text { UMHC } \\
\text { UMHC }\end{array}$ & COND & 117 & 1 \\
\hline $\begin{array}{ll}F B P & 1 A \\
F B P & 1 A\end{array}$ & 8703 & 83.6 & UMHC & COND & 82.825 & 4 \\
\hline FBP IA & 8704 & $\begin{array}{l}99.1 \\
74.3\end{array}$ & UMHC & $\begin{array}{l}\text { COND } \\
\text { COND }\end{array}$ & $\begin{array}{l}99.1 \\
73.5\end{array}$ & $\begin{array}{l}1 \\
2\end{array}$ \\
\hline $\begin{array}{l}\text { FBP } 1 A \\
\text { FBP }\end{array}$ & $\begin{array}{l}8801 \\
8901\end{array}$ & $\begin{array}{r}74.3 \\
109\end{array}$ & UMHC & COND & 109 & | \\
\hline
\end{tabular}

Plot File of Laboratory Analyses at FBP Monitoring Wells - Page 9 
DATA RETRIEVED 7/2/91

\begin{tabular}{|c|c|c|c|c|c|c|c|}
\hline WELLL & & QTA & $\begin{array}{l}\text { MAXINUT } \\
\text { RESULT } \\
\end{array}$ & UNITS & TESTNAME & $\begin{array}{l}\text { MEAN } \\
\text { RESULT }\end{array}$ & OBS \\
\hline $\begin{array}{l}\text { FBP } 1 / \\
\text { FBP } 1 / \\
\text { FBP } 1 /\end{array}$ & $\begin{array}{l}\text { IA } \\
1 A \\
1 A\end{array}$ & $\begin{array}{l}8902 \\
9001 \\
9101 \\
\end{array}$ & $\begin{array}{r}1200 \\
90 \\
98 \\
\end{array}$ & $\begin{array}{l}\text { UMHC } \\
\text { UMHC } \\
\text { UMHC }\end{array}$ & \begin{tabular}{l|} 
COND \\
COND \\
COND \\
\end{tabular} & $\begin{array}{r}1200 \\
89 \\
98\end{array}$ & $\begin{array}{l}1 \\
2 \\
2 \\
\end{array}$ \\
\hline FBP 2 & $2 A$ & 8403 & 46.4 & UMHC & COND & 46.4 & 1 \\
\hline $\begin{array}{l}\text { FBP } 2 \\
\text { FBP } 2\end{array}$ & ¿A & $\begin{array}{l}8404 \\
8501\end{array}$ & $\begin{array}{l}53.6 \\
68.3\end{array}$ & $\begin{array}{l}\text { UMHC } \\
\text { UMHC }\end{array}$ & $\begin{array}{l}\text { COND } \\
\text { COND }\end{array}$ & $\begin{array}{l}53.6 \\
68.3\end{array}$ & $\begin{array}{l}1 \\
1\end{array}$ \\
\hline FBP 2 & A & 8502 & 55 & UMHC & COND & 55 & 1 \\
\hline FBP 2 & DA & 8503 & 68.8 & UMHC & COND & 68.8 & 1 \\
\hline FBP 2 & $2 A$ & 8504 & 250 & UMHC & COND & 250 & 1 \\
\hline FBP 2 & EA & 86Q1 & 98.8 & UMHC & COND & 98.8 & 1 \\
\hline FBP 2 & $2 A$ & 8602 & 73.2 & UMHC & COND & 73.2 & 1 \\
\hline $\begin{array}{l}\text { FBP } 2 \\
\text { FBP } 2\end{array}$ & $2 A$ & 8603 & 49 & UMHC & $\begin{array}{l}\text { COND } \\
\text { COND }\end{array}$ & $\begin{array}{r}49 \\
48\end{array}$ & 11 \\
\hline FBP 2 & $2 A$ & 8701 & $\begin{array}{l}44.0 \\
38.4\end{array}$ & UMHC & COND & 38.4 & 1 \\
\hline FBP 2 & $2 A$ & 8702 & 43.9 & UMHC & COND & 43.8 & 1 \\
\hline FBP 2 & 2A & 8703 & 79.8 & UMHC & COND & 79.8 & 1 \\
\hline FBP 2 & $2 A$ & 8704 & 106 & UMHIC & COND & 106 & $\begin{array}{l}2 \\
1\end{array}$ \\
\hline $\begin{array}{ll}\text { FBP } & 2 \\
\text { FBP } & 2\end{array}$ & 2A & $\begin{array}{l}8801 \\
8901\end{array}$ & $\begin{array}{r}65 \\
45.2\end{array}$ & $\begin{array}{l}\text { UMHC } \\
\text { UMHC }\end{array}$ & $\begin{array}{l}\text { COND } \\
\text { COND }\end{array}$ & $\begin{array}{r}65 \\
45.2\end{array}$ & $\begin{array}{l}1 \\
1\end{array}$ \\
\hline FBP 2 & $2 A$ & 8902 & $\begin{array}{r}35 \\
35\end{array}$ & UMHC & COND & 35 & 1 \\
\hline FBP 2 & 2A & 8001 & 38) & UMHC & COND & 38 & 1 \\
\hline FBP 2 & 2A & 9101 & 50 & UMHC & COND & 50 & 1 \\
\hline FBP 3. & $3 A$ & 8404 & 52.1 & UMHC & COND & 52.1 & 1 \\
\hline FBP 3 & $3 A$ & 8501 & 130.5 & UMHC & COND & 130.5 & 1 \\
\hline FBP 3 & $3 A$ & 8502 & 54.9 & UMHC & COND & 54.9 & 1 \\
\hline FBP 3 & $3 A$ & 8503 & 66.3 & UMHC & COND & 66.3 & 1 \\
\hline FBP 3, & $3 A$ & 8504 & 70 & UMHC & COND & 70 & 1 \\
\hline FBP 3 & $3 A$ & 8601 & 78.5 & UMHC & COND & 78.5 & 1 \\
\hline FBP 3. & $3 A$ & 8602 & 77.4 & UMHC & COND & 77.4 & 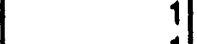 \\
\hline FBP 3. & $3 A$ & $86 \curvearrowright 3$ & 62.4 & UMHC & COND & 62.4 & $\begin{array}{l}1 \\
0\end{array}$ \\
\hline FBP 3. & $3 A$ & 8604 & 67.6 & UMHC & COND & 64.45 & 2 \\
\hline FBP 3 & BA & $\begin{array}{l}8701 \\
8702\end{array}$ & 58.8 & UMHC & $\begin{array}{l}\text { COND } \\
\text { COND }\end{array}$ & $\begin{array}{l}58.8 \\
55.4\end{array}$ & $\begin{array}{l}1 \\
1\end{array}$ \\
\hline $\begin{array}{l}\text { FBP } \\
\text { FBP } \\
\text { FBP }\end{array}$ & $3 A$ & $\begin{array}{l}8702 \\
8703\end{array}$ & $\begin{array}{l}55.4 \\
51.5\end{array}$ & UMHC & COND & 51.5 & 1 \\
\hline FBP 3 & $3 A$ & 8801 & 50 & UMHC & COND & 50 & I \\
\hline FBP 3 & $3 A$ & 8901 & 61.3 & UMHC & COND & 61.3 & $\begin{array}{l}1 \\
1\end{array}$ \\
\hline FBP 3 & $3 A$ & 8902 & 60 & UMHC & COND & 60| & 1 \\
\hline FBP 3 & $3 A$ & 9001 & 55 & UMHC & COND & 55 & 1 \\
\hline FBP 3 & $3 A$ & 9101 & 52 & UMHC & COND & 52 & 1 \\
\hline$\overline{F B P \quad 4}$ & & $85 Q 1$ & 30.3 & UMHC & COND & 30.3 & 1 \\
\hline FBP 4 & & $85 Q 2$ & 17.6 & UMHC & COND & 17.6 & \\
\hline FBP 4 & & 8503 & 18.6 & UMHC & COND & 18.6 & 1 \\
\hline FBP 4 & 4 & 8504 & 19 & UMHC & COND & 18.5 & 2 \\
\hline FBP 4 & 4 & $66 Q 1$ & 19.82 & UMHC & ND & 19.82 & 1 \\
\hline FBP 4 & 4 & $86 Q 2$ & 21.29 & UMHC & ND & 21.29 & 1 \\
\hline FBP 4 & 4 & 8603 & 18.3 & UMHC & COND & 18.3 & 1 \\
\hline FBP 4 & 4 & $86 Q 4$ & 21.6 & UMHC & ND & 21.6 & 1 \\
\hline FBP 4 & 4 & $87 Q 1$ & 20.7 & UMHC & ND & 20.7 & 1 \\
\hline FBP 4 & 4 & $87 Q 2$ & 22.8 & UMHS & ND & 22,35 & 2 \\
\hline FBP 4 & 4 & 8703 & 22.4 & UMHCC & ND & 22.4 & 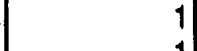 \\
\hline FBP 4 & 4 & 8704 & 25.6 & UMHC & ND & 25.6 & 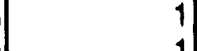 \\
\hline FBP 4 & 4 & 8801 & 20 & UMHC & ND & 20 & 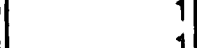 \\
\hline FBP 4 & 4 & 8901 & 31.8 & UMHC & ND & 31.8 & 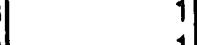 \\
\hline FBP 4 & 4 & 8902 & 20 & UMHC & ND & 20 & 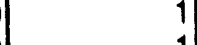 \\
\hline P 4 & 4 & 9001 & 30 & UMHC & ND & 30 & 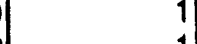 \\
\hline FBP 4 & 4 & 9101 & 22 & UMHC & & 22 & 1 \\
\hline FBP 1 & $1 \mathrm{~A}$ & 8404 & 0.0021 & MMY & CORA & 0.0021 & 1 \\
\hline FBP 2 & $2 A$ & 8404 & 0.0038 & MMY & CORA & 0.0038 & 1 \\
\hline FBP 3 & $3 A$ & 8404 & 0.0031 & MMY & COAA & 0.0031 & 1 \\
\hline FBP 1 & $1 \mathrm{~A}$ & 8403 & .0 .004 & MGL & CA & -0.004 & . \\
\hline FBP 1 & $1 A$ & 8404 & -0.004 & MGL & C & -0.004 & \\
\hline P 1 & $1 A$ & 8501 & .0 .004 & MGL & & 0.004 & 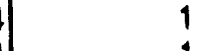 \\
\hline FBP 1 & $1 A$ & 1870 & -0.004 & MGL & & -0.004 & \\
\hline
\end{tabular}

Plot File of Laboratory Analyses at FBP Monitoring Wells - Page 10 
DATA RETRIEVED 7/2/91

\begin{tabular}{|c|c|c|c|c|c|c|}
\hline WELL. & arR & $\begin{array}{l}\text { MAXINUUI } \\
\text { RESULT }\end{array}$ & UNITS & TESTNAME & $\begin{array}{l}\text { NEAN } \\
\text { RESULT }\end{array}$ & OBg \\
\hline $\begin{array}{ll}F B P & 1 A \\
\text { FBP } & 1 A \\
\text { FBP } & 1 A\end{array}$ & $\begin{array}{l}8902 \\
9001 \\
8101\end{array}$ & $\begin{array}{r}-0.004 \\
0.004 \\
0.0048 \\
\end{array}$ & $\begin{array}{l}\text { MGL } \\
\text { MGL } \\
\text { MGL } \\
\end{array}$ & $\begin{array}{l}\mathrm{CA} \\
\mathrm{CA} \\
\mathrm{OA} \\
\end{array}$ & $\begin{array}{l}.0 .004 \\
0.004 \\
0.0045\end{array}$ & $\begin{array}{l}1 \\
2 \\
2 \\
\end{array}$ \\
\hline $\begin{array}{ll}F B P & 2 A \\
F B P & 2 A\end{array}$ & $\begin{array}{l}84 C 2 \\
8404\end{array}$ & $\begin{array}{l}-0.004 \\
-0.004\end{array}$ & $\begin{array}{l}\text { MGL. } \\
\text { MGL. }\end{array}$ & $\begin{array}{l}\text { CA } \\
\text { CA }\end{array}$ & $\begin{array}{l}.0,004 \\
.0 .004\end{array}$ & 1 \\
\hline $\begin{array}{ll}\text { FBP } & 2 A \\
\text { FBP } & 2 A\end{array}$ & $\begin{array}{l}8501 \\
8701\end{array}$ & $\begin{array}{l}-0.004 \\
-0.004\end{array}$ & $\begin{array}{l}\text { MGL } \\
\text { MGL. }\end{array}$ & $\begin{array}{l}\text { OA } \\
\text { CR }\end{array}$ & $\begin{array}{l}-0.004 \\
.0 .004\end{array}$ & $\begin{array}{l}1 \\
1\end{array}$ \\
\hline FBP 2A & 8902 & -0.004 & MGL & CR & $.0,004$ & 1 \\
\hline $\begin{array}{ll}\text { FBP } & 2 A \\
\text { FBP } & 2 A\end{array}$ & $\begin{array}{l}9001 \\
9101\end{array}$ & $\begin{array}{r}-0.004 \\
-0.004\end{array}$ & $\begin{array}{l}\text { MGL } \\
\text { MGL }\end{array}$ & $\begin{array}{l}\mathrm{CR} \\
\mathrm{CR}\end{array}$ & $\begin{array}{r}-0.004 \\
-0.004\end{array}$ & $\begin{array}{l}1 \\
1\end{array}$ \\
\hline FBP $3 A$ & 8403 & .0 .004 & MGL & CR & -0.004 & 1 \\
\hline FBP 3A & 8404 & -0.004 & MGL & CR & -0.004 & 1 \\
\hline FBP 3A & 85.01 & $-0,004$ & MGL & CR & -0.004 & 1 \\
\hline FBP 3A & 87Q1 & -0.004 & MGL & CR & -0.004 & 1 \\
\hline $\begin{array}{ll}F B P & 3 A \\
\text { FBP } & 3 A\end{array}$ & 8902 & $\begin{array}{l}-0.004 \\
.0 .004\end{array}$ & MGL & $\mid \begin{array}{l}C A \\
C R\end{array}$ & $\begin{array}{l}-0.004 \\
-0.004\end{array}$ & $\begin{array}{l}1 \\
1\end{array}$ \\
\hline FBP $3 A$ & 9101 & 0.0049 & MGL & $\mathrm{CA}$ & 0.0049 & 1 \\
\hline FBP 4 & 8501 & -0.004 & MGL & CA & -0.004 & 1 \\
\hline FBP 4 & 8503 & -0.004 & MGL & CR & -0.004 & 1 \\
\hline FBP 4 & 8504 & $-0,004$ & MGL & CA & -0.004 & 2 \\
\hline FBP 4 & 86Q1 & -0.004 & MGL & CA & $\begin{array}{l}-0.004 \\
.0 .004\end{array}$ & $\begin{array}{l}1 \\
1\end{array}$ \\
\hline $\begin{array}{ll}\text { FBP } & 4 \\
\text { FBP } & 4\end{array}$ & $\begin{array}{l}8701 \\
8902\end{array}$ & $\begin{array}{l}0.004 \\
0.0119\end{array}$ & $\begin{array}{l}\text { MGL } \\
\text { MGL }\end{array}$ & $\begin{array}{l}\mathrm{CH} \\
\mathrm{CA}\end{array}$ & 0.0119 & $\begin{array}{l}1 \\
1\end{array}$ \\
\hline FBP 4 & 9001 & -0.004 & MaL & CR & -0.004 & 1 \\
\hline FBP 4 & 9101 & -0.004 & MGL & CR & -0.004 & 1 \\
\hline FBP IA & 8403 & -0.004 & MGL & $\mathrm{CU}$ & -0.004 & 2 \\
\hline FBP 1A & 8404 & -0.004 & MGL & $\mathrm{CU}$ & -0.004 & 1 \\
\hline FBP 2A & 8403 & -0.004 & MGL & $\mathrm{CU}$ & -0.004 & 1 \\
\hline FBP $2 A$ & 8404 & 0.007 & MGL & $\mathrm{Cu}$ & 0.007 & 1 \\
\hline FBP 3A & 8403 & .0 .004 & MGL & $\mathrm{CU}$ & -0.004 & 1 \\
\hline FBP $3 A$ & 8404 & 0.009 & MGL & Cu & 0.009 & 1 \\
\hline FBP $1 A$ & 8403 & -0.005 & MGL & CYN & -0.005 & 1 \\
\hline FBP $1 \mathrm{~A}$ & 8404 & .0 .005 & MGL & CYN & -0.005 & 1 \\
\hline FBP $2 A$ & 8403 & -0.005 & MGL & CYN & -0.005 & 1 \\
\hline FBP 2A & 8404 & -0.005 & MGL & CYN & -0.005 & 1 \\
\hline FBP $3 A$ & 8403 & .0 .005 & MGL & CYN & -0.005 & 1 \\
\hline FBP $3 A$ & 8404 & -0.005 & MGL & CYN & -0.005 & 1 \\
\hline FBP 1A & 8703 & -0.001 & MGL & C13DCP & -0.001 & 1 \\
\hline FBP $1 A$ & 8504 & -0.01 & MGL & $\mathrm{C} 2 \mathrm{H} 3 \mathrm{CL}$ & -0.01 & 1 \\
\hline FBP $1 A$ & 8903 & -0.001 & MGL & $\mathrm{C} 2 \mathrm{H} 3 \mathrm{CL}$ & -0.001 & 1 \\
\hline FBP 1A & 9001 & -0.001 & $M G L$ & $\mathrm{C} 2 \mathrm{H} 3 \mathrm{CL}$ & -0.001 & 2 \\
\hline FBP 2A & 8504 & -0.01 & MGL & $\mathrm{C} 2 \mathrm{H} 3 \mathrm{CL}$ & -0.01 & 1 \\
\hline FBP $2 A$ & 8903 & -0.001 & MGL & $\mathrm{C} 2 \mathrm{H} 3 \mathrm{CL}$ & -0.001 & 1 \\
\hline FBP $2 A$ & 9001 & .0 .001 & MGL & $\mathrm{C} 2 \mathrm{H} 3 \mathrm{CL}$ & .0 .001 & 1 \\
\hline FBP $3 A$ & 8504 & -0.01 & MGL & C2H3CL & -0.01 & 1 \\
\hline FBP $3 A$ & 8903 & -0.001 & MGL & $\mathrm{C} 2 \mathrm{H} 3 \mathrm{CL}$ & -0.001 & 2 \\
\hline FBP $3 A$ & 9001 & -0.001 & $M G L$ & $\mathrm{C} 2 \mathrm{H} 3 \mathrm{CL}$ & -0.001 & 1 \\
\hline$\overline{F B P} 4$ & 8524 & -0.01 & MGL & $\mathrm{C} 2 \mathrm{H} 3 \mathrm{CL}$ & -0.01 & $\overline{1}$ \\
\hline FBP 4 & 8903 & -0.001 & MCL & $\mathrm{C} 2 \mathrm{H} 3 \mathrm{CL}$ & -0.001 & 1 \\
\hline FBP 4 & 9001 & -0.001 & MGL & $\mathrm{C} 2 \mathrm{H} 3 \mathrm{CL}$ & -0.001 & 1 \\
\hline FBP 1A & 8504 & .0 .01 & MGL & C2H5CL & .0 .01 & 1 \\
\hline FBP $1 A$ & 8703 & -0.001 & MGL & $\mathrm{C} 2 \mathrm{H} 5 \mathrm{CL}$ & -0.001 & 1 \\
\hline FBP $1 A$ & 8903 & -0.001 & MGL & $\mathrm{C} 2 \mathrm{H} 5 \mathrm{CL}$ & -0.001 & \\
\hline FBP 1A & 9001 & .0 .001 & MGL & $\mathrm{C} 2 \mathrm{H} 5 \mathrm{CL}$ & -0.001 & 2 \\
\hline FBP 2A & 8504 & .0 .01 & MGL & C2H5CL & -0.01 & 1 \\
\hline FBP 2A & 8903 & -0.001 & MGL & $\mathrm{C} 2 \mathrm{H} 5 \mathrm{Cl}$. & -0.001 & 1 \\
\hline FBP $2 A$ & 9001 & -0.001 & MGL & $\mathrm{C} 2 \mathrm{H} 5 \mathrm{CL}$ & .0 .001 & 1 \\
\hline FBP $3 A$ & 8504 & -0.01 & MGL & C2H5CL. & -0.01 & 1 \\
\hline FBP $3 A$ & 8903 & -0.001 & MGL & $\mathrm{C} 2 \mathrm{H} 5 \mathrm{CL}$ & -0.001 & 2 \\
\hline FBP $3 A$ & 9001 & -0.001 & MGL & $\mathrm{C} 2 \mathrm{H} 5 \mathrm{CL}$ & -0.001 & 1 \\
\hline FBP 4 & 8504 & -0.01 & MGL & C2H5CL & .0 .01 & 1 \\
\hline $3 P 4$ & 8903 & .0 .001 & $M G L$ & $\mathrm{C} 2 \mathrm{H} 5 \mathrm{CL}$ & -0.001 & 1 \\
\hline FBP 4 & Q1 & -0.001 & MGL & $\mathrm{C} 2 \mathrm{H} 5 \mathrm{CL}$ & -0.0011 & \\
\hline
\end{tabular}

Plot File of Laboratory Analyses at FBP Monitoring Wells - Page 11 
DATA RETRIEVED 7/2/91

\begin{tabular}{|c|c|c|c|c|c|c|}
\hline WELL & aTR & $\begin{array}{l}\text { MAXIINUM } \\
\text { BEgULT }\end{array}$ & UNITE & TESTNAME & $\begin{array}{l}\text { WEAN } \\
\text { RESUL,T }\end{array}$ & Ogs \\
\hline $\begin{array}{ll}F B P & 1 A \\
F B P & 1 A \\
F B P & 1 A\end{array}$ & $\begin{array}{l}8504 \\
8903 \\
0001\end{array}$ & $\begin{array}{r}-0.006 \\
-0.001 \\
.0 .001\end{array}$ & $\begin{array}{l}\text { MaL } \\
\text { MaL } \\
\text { MGL }\end{array}$ & $\begin{array}{l}\mathrm{C} 6 \mathrm{HE} \\
\mathrm{C} 6 \mathrm{H} 6 \\
\mathrm{O} 6 \mathrm{H} 6 \mathrm{6}\end{array}$ & \begin{tabular}{|r|}
0.005 \\
0.001 \\
.0 .001
\end{tabular} & $\begin{array}{l}1 \\
1 \\
2 \\
\end{array}$ \\
\hline $\begin{array}{ll}\text { FB̈P } & 2 A \\
\text { FBP } & 2 A \\
\text { FBP } & 2 A\end{array}$ & $\begin{array}{l}8504 \\
8903 \\
9001\end{array}$ & $\begin{array}{l}.0,005 \\
.0,001 \\
.0,001\end{array}$ & $\begin{array}{l}\text { MaL } \\
\text { MGL } \\
\text { MGL }\end{array}$ & $\begin{array}{l}\mathrm{C} 6 \mathrm{H} 6 \\
\mathrm{C} 6 \mathrm{H} 6 \\
\mathrm{C} 6 \mathrm{H} 6\end{array}$ & $\begin{array}{l}-0.005 \\
.0 .001 \\
-0.001\end{array}$ & $\begin{array}{l}1 \\
1 \\
1\end{array}$ \\
\hline $\begin{array}{ll}F B P & 3 A \\
F B P & 3 A \\
F B P & 3 A\end{array}$ & $\begin{array}{l}8504 \\
8903 \\
8001\end{array}$ & $\begin{array}{l}-0,006 \\
-0,001 \\
-0,001\end{array}$ & $\begin{array}{l}\text { MGL } \\
\text { MGL } \\
\text { MGL }\end{array}$ & $\begin{array}{l}\mathrm{C} 6 \mathrm{H} 6 \\
\mathrm{C} 6 \mathrm{H} 6 \\
\mathrm{C} 6 \mathrm{H} 6 \\
\end{array}$ & $\begin{array}{l}.0 .005 \\
.0 .001 \\
.0 .001\end{array}$ & $\begin{array}{l}1 \\
2 \\
1\end{array}$ \\
\hline $\begin{array}{l}\text { FGP } 4 \\
\text { FBP } 4 \\
\text { FBP } 4\end{array}$ & $\begin{array}{l}8504 \\
8903 \\
9001\end{array}$ & $\begin{array}{l}-0.006 \\
-0.001 \\
-0.001\end{array}$ & $\begin{array}{l}\text { MGL } \\
\text { MGL } \\
\text { MGL }\end{array}$ & $\begin{array}{l}\mathrm{C} 6 \mathrm{H} 6 \\
\mathrm{C} 6 \mathrm{H} 6 \\
\mathrm{C} 6 \mathrm{H} 6\end{array}$ & $\begin{array}{l}.0 .005 \\
.0,001 \\
.0,001\end{array}$ & $\begin{array}{l}1 \\
1 \\
1\end{array}$ \\
\hline $\begin{array}{ll}\text { FBP } & 1 A \\
\text { FBP } & 1 A \\
\text { FBP } & 1 A \\
\text { FBP } & 1 A\end{array}$ & $\begin{array}{l}8504 \\
8703 \\
8903 \\
9001\end{array}$ & $\begin{array}{l}-0.005 \\
-0.001 \\
-0.001 \\
-0.001\end{array}$ & $\begin{array}{l}\text { MGL } \\
\text { MGL } \\
\text { MGL } \\
\text { MGL }\end{array}$ & $\begin{array}{l}\text { DBACLM } \\
\text { DBACLM } \\
\text { DBRCLM } \\
\text { DBRCLM }\end{array}$ & $\begin{array}{l}-0.005 \\
-0.001 \\
.0 .001 \\
.0 .001\end{array}$ & $\begin{array}{l}1 \\
1 \\
1 \\
2\end{array}$ \\
\hline $\begin{array}{ll}\text { FBP } & 2 A \\
\text { FBP } & 2 A \\
\text { FBP } & 2 A\end{array}$ & $\begin{array}{l}8504 \\
8903 \\
9001\end{array}$ & $\begin{array}{l}-0.005 \\
-0.001 \\
-0.001\end{array}$ & $\begin{array}{l}\text { MGL } \\
\text { MGL } \\
\text { MGL }\end{array}$ & $\begin{array}{l}\text { DBRCLM } \\
\text { DBRCLM } \\
\text { DBRCL.M }\end{array}$ & $\begin{array}{l}-0.005 \\
-0.001 \\
.0 .001\end{array}$ & $\begin{array}{l}1 \\
1 \\
1\end{array}$ \\
\hline $\begin{array}{ll}F B P & 3 A \\
\text { FBP } & 3 A \\
\text { FBP } & 3 A\end{array}$ & $\begin{array}{l}8504 \\
8903 \\
9001\end{array}$ & $\begin{array}{l}-0.005 \\
-0.001 \\
.0 .001\end{array}$ & $\begin{array}{l}\text { MGL } \\
\text { MGL } \\
\text { MGL }\end{array}$ & $\begin{array}{l}\text { DBRCLM } \\
\text { DBRCLM } \\
\text { DBRCLM }\end{array}$ & $\begin{array}{l}-0.005 \\
-0.001 \\
.0 .001\end{array}$ & $\begin{array}{l}1 \\
2 \\
1 \\
\end{array}$ \\
\hline $\begin{array}{ll}\text { FBP } & 4 \\
\text { FBP } & 4 \\
\text { FBP } & 4\end{array}$ & $\begin{array}{l}8501 \\
8903 \\
9001\end{array}$ & $\begin{array}{l}-0.005 \\
-0.001 \\
-0.001\end{array}$ & $\begin{array}{l}\text { MGL } \\
\text { MGL } \\
\text { MGL }\end{array}$ & $\begin{array}{l}\text { DBRCLM } \\
\text { DBRCLM } \\
\text { DBRCLM }\end{array}$ & $\begin{array}{l}.0 .005 \\
.0 .001 \\
.0,001 \\
\end{array}$ & $\begin{array}{l}1 \\
1 \\
1 \\
\end{array}$ \\
\hline $\begin{array}{ll}F B P & 1 A \\
F B P & 1 A\end{array}$ & $\begin{array}{l}8403 \\
8404\end{array}$ & $\begin{array}{r}-5 \\
5.8\end{array}$ & $\begin{array}{l}\text { MGL } \\
\text { MGL }\end{array}$ & $\begin{array}{l}D O C \\
D O C\end{array}$ & $\begin{array}{r}.5 \\
5.8\end{array}$ & $\begin{array}{l}1 \\
1 \\
1\end{array}$ \\
\hline $\begin{array}{ll}F B P & 2 A \\
F B P & 2 A\end{array}$ & $\begin{array}{l}8403 \\
8404\end{array}$ & \begin{tabular}{r|}
5 \\
.5
\end{tabular} & $\begin{array}{l}\text { MGL } \\
\text { MGL }\end{array}$ & $\begin{array}{l}\text { DOC } \\
D O C\end{array}$ & $\begin{array}{r}3.75 \\
-5 \\
\end{array}$ & $\begin{array}{l}2 \\
1\end{array}$ \\
\hline FBP $3 A$ & 8403 & 7 & MaL & DOC & 7 & $\begin{array}{l}1 \\
1\end{array}$ \\
\hline FBP $3 A$ & 8404 & -5 & MGL & IDOC & .5 & 1 \\
\hline FBP 1A & 8404 & .0 .00004 & $M G L$ & ENDAN & .0 .00004 & 1 \\
\hline $\begin{array}{ll}\text { FBP } & 1 A \\
\text { FBP } & 1 A \\
\end{array}$ & $\begin{array}{l}8501 \\
9101 \\
\end{array}$ & $\begin{array}{l}-0.00004 \\
-0.00001 \\
\end{array}$ & $\begin{array}{l}\text { MGL } \\
\text { MGL }\end{array}$ & $\begin{array}{l}\text { ENDRN } \\
\text { ENDRN }\end{array}$ & $\begin{array}{l}-0.00004 \\
-0.00001 \\
\end{array}$ & $\begin{array}{l}2 \\
2 \\
\end{array}$ \\
\hline FBP 2A & 8404 & .0 .00004 & MGL & ENDAN & .0 .00004 & 1 \\
\hline FBP $2 A$ & 8501 & -0.00004 & $\begin{array}{l}\text { MGL } \\
\text { MGL }\end{array}$ & $\begin{array}{l}\text { ENDAN } \\
\text { ENDRN }\end{array}$ & $\begin{array}{l}-0.00004 \\
-0.00001\end{array}$ & $\begin{array}{l}1 \\
1\end{array}$ \\
\hline FBP 2A & 9101 & $\frac{-0.00001}{.000004}$ & $\frac{M G L}{M G L}$ & ENDHN & $\frac{-0.00001}{.0 .00004}$ & $\frac{1}{1}$ \\
\hline $\begin{array}{ll}\text { FBP } & 3 A \\
\text { FBP } & 3 A\end{array}$ & $\begin{array}{l}84 Q 4 \\
8501\end{array}$ & $\begin{array}{l}-0.00004 \\
-0.00004\end{array}$ & $\begin{array}{l}\text { MGL } \\
\text { MGL }\end{array}$ & $\begin{array}{l}\text { ENDAN } \\
\text { ENDRN }\end{array}$ & $\begin{array}{l}-0.00004 \\
-0.00004\end{array}$ & 1 \\
\hline FBP $3 A$ & 9101 & -0.00001 & MGL & ENDRN & $-0,00001$ & 1 \\
\hline FBP 4 & $85 Q 1$ & -0.00004 & MGL & ENDAN & $-0,00004$ & 1 \\
\hline FBP 4 & 8503 & -0.00004 & MGL & ENDRN & -0.00004 & 1 \\
\hline FBP 4 & 8504 & -0.00004 & MGL & ENDRN & -0.00004 & 2 \\
\hline FBP 4 & 8601 & .0 .00004 & $M G L$ & ENDRN & $-0,00004$ & \\
\hline FBP 4 & 9101 & .0 .00001 & $M G L$ & ENDRN & $.0,00001$ & 1 \\
\hline FBP $1 A$ & 8504 & -0.005 & MGL & ETC6H5 & .0 .005 & $\overline{1}$ \\
\hline FBP $1 A$ & 8903 & -0.001 & MGL & ETC6H5 & -0.001 & 1 \\
\hline FBP IA & 9001 & .0 .001 & MGL & ETC6H5 & $-0,001$ & 2 \\
\hline FBP $2 A$ & 8504 & .0 .005 & MGL & ETC6H5 & .0 .005 & 1 \\
\hline FBP 2A & 8903 & -0.001 & MGL & ETC6H5 & .0 .001 & 1 \\
\hline FBP 2A & 9001 & -0.001 & MGL & ETC6H5 & .0 .001 & 1 \\
\hline FBP $3 A$ & 8504 & -0.005 & $M G L$ & ETC6H5 & .0 .005 & 1 \\
\hline FBP $3 A$ & 8903 & -0.001 & MGL & ETC6H5 & -0.001 & 2 \\
\hline FBP $3 A$ & 9001 & -0.001 & MGL & ETC6H5 & -0.001 & 1 \\
\hline FBP 4 & 8504 & -0.005 & MGL & ETC6H5 & .0 .005 & 1 \\
\hline FBP 4 & 8903 & -0.001 & MGL & ETC6H5 & -0.001 & 1 \\
\hline FBP 4 & 9001 & -0.001 & MGL & ETC6H5 & -0.001 & 1 \\
\hline FBP IA & 8403 & 0.1 & MGL & $F$ & 0.1 & 1 \\
\hline FBP $1 A$ & 8404 & -0.1 & MGL & $F$ & -0.1 & 2 \\
\hline FBP $1 A$ & 8501 & 0.11 & MGL & & 0.11 & \\
\hline FBP 1A & & 0.14 & MGL & $F$ & 0.14 & 1 \\
\hline FBP $1 A$ & & $\begin{array}{l}-0.1 \\
-0.1\end{array}$ & $\begin{array}{l}M G L \\
M G L\end{array}$ & $F$ & $\begin{array}{l}-0.1 \\
-0.1\end{array}$ & $\begin{array}{l}1 \\
2\end{array}$ \\
\hline FBP 1A & & .0 & & & & \\
\hline
\end{tabular}

Plot Flle of Laboratory Analyses at FBP Monitoring Wells * Page 12 
DATA RETAIEVED 7/2/91

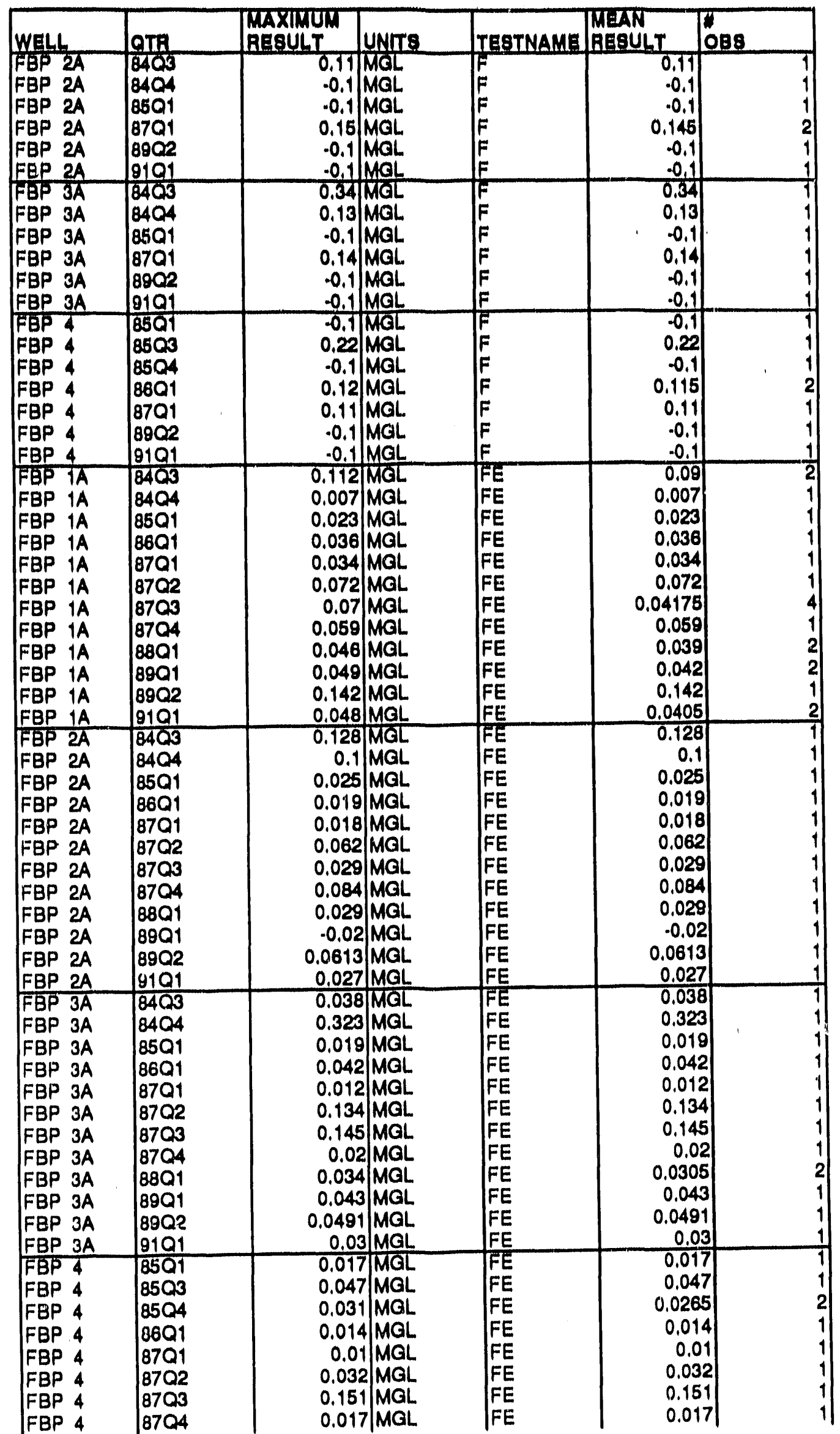

Plot File of Laboratory Analyses at FBP Monitoring Wells - Page 13 
DATA RETAIEVED 7/2/91

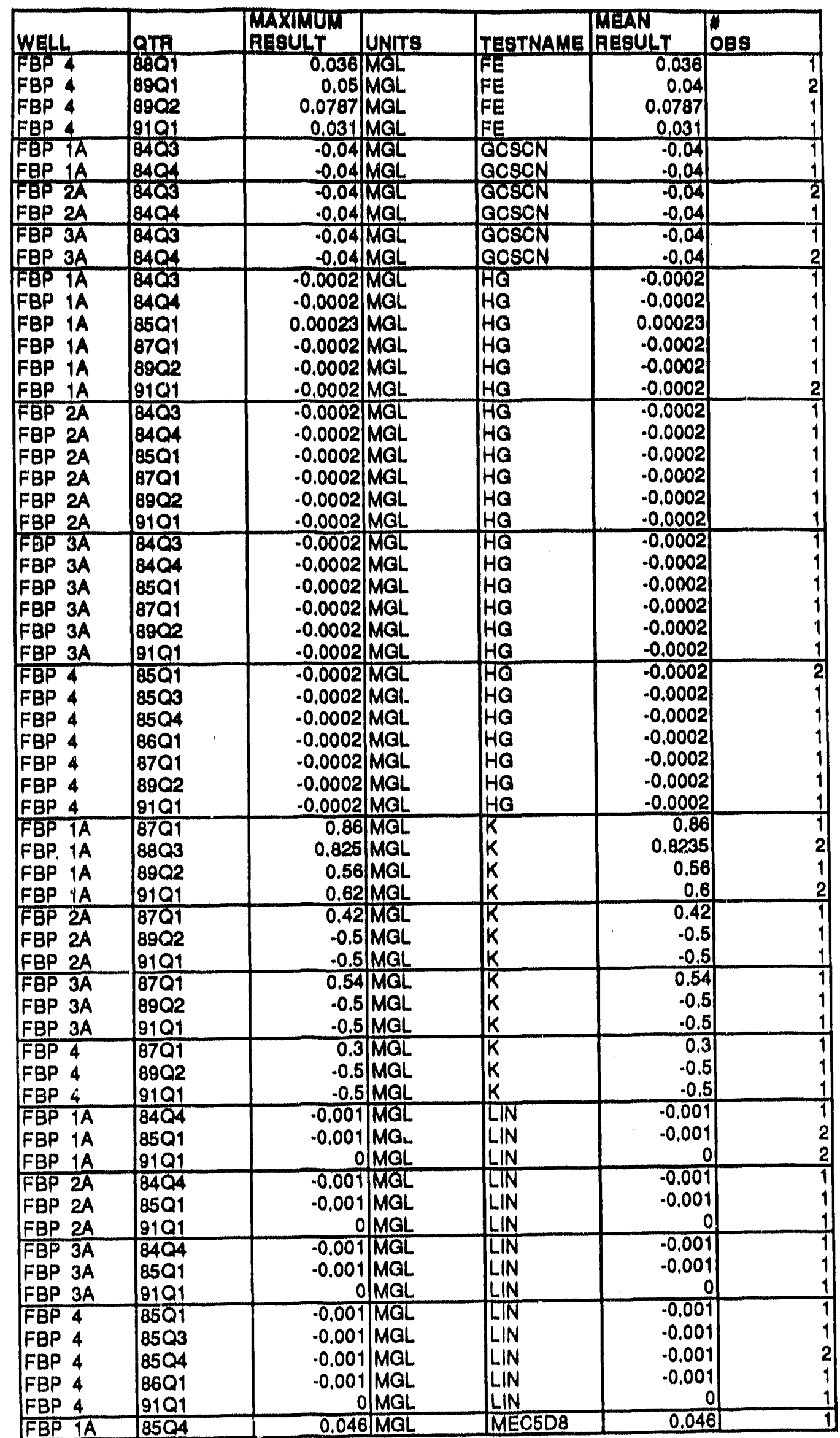

Plot Flle of Laboratory Analyses at FBP Monitoring Wolls - Page 14 
DATA RETAIEVED 7/2/91

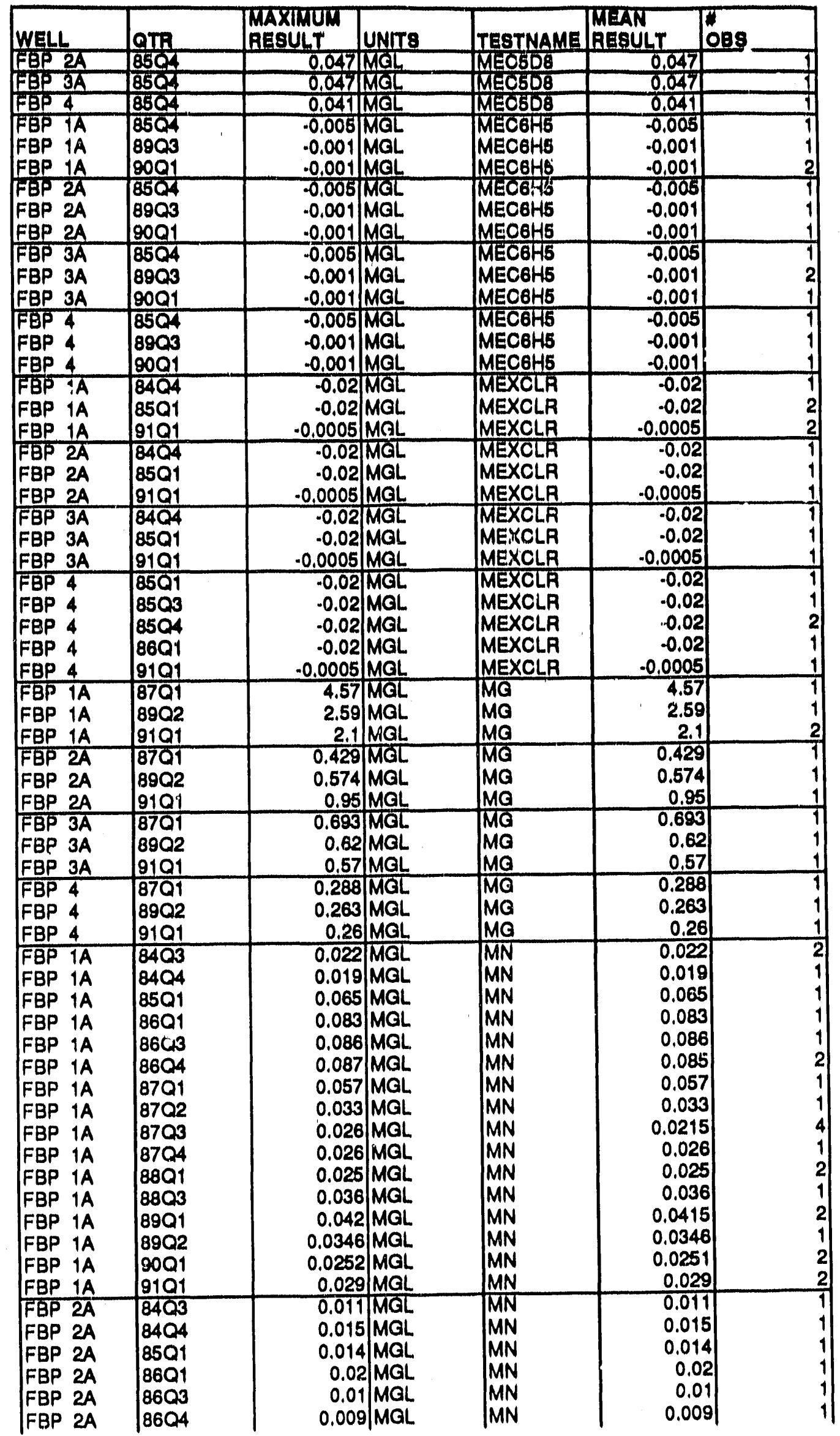

Plot Flle of Laboratory Analyses at FBP Monitoring Wells - Page 15 
DATA RETRIEVED 7/2/91

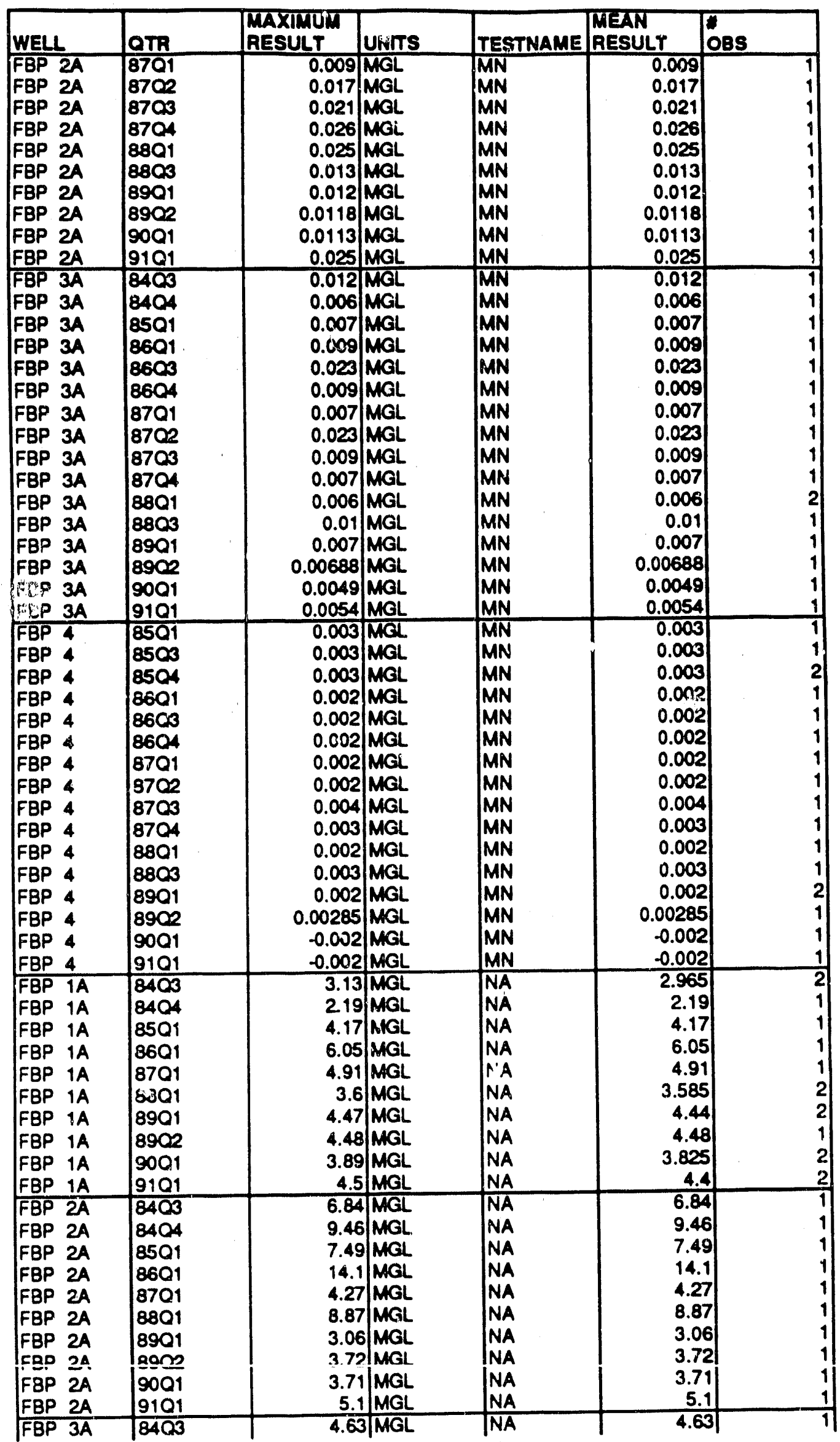

Plot File of Laboratory Anthatyses at FBP Monitoring Wolls - Page 16 
DATA RETRIEVED $7 / 2 / 91$

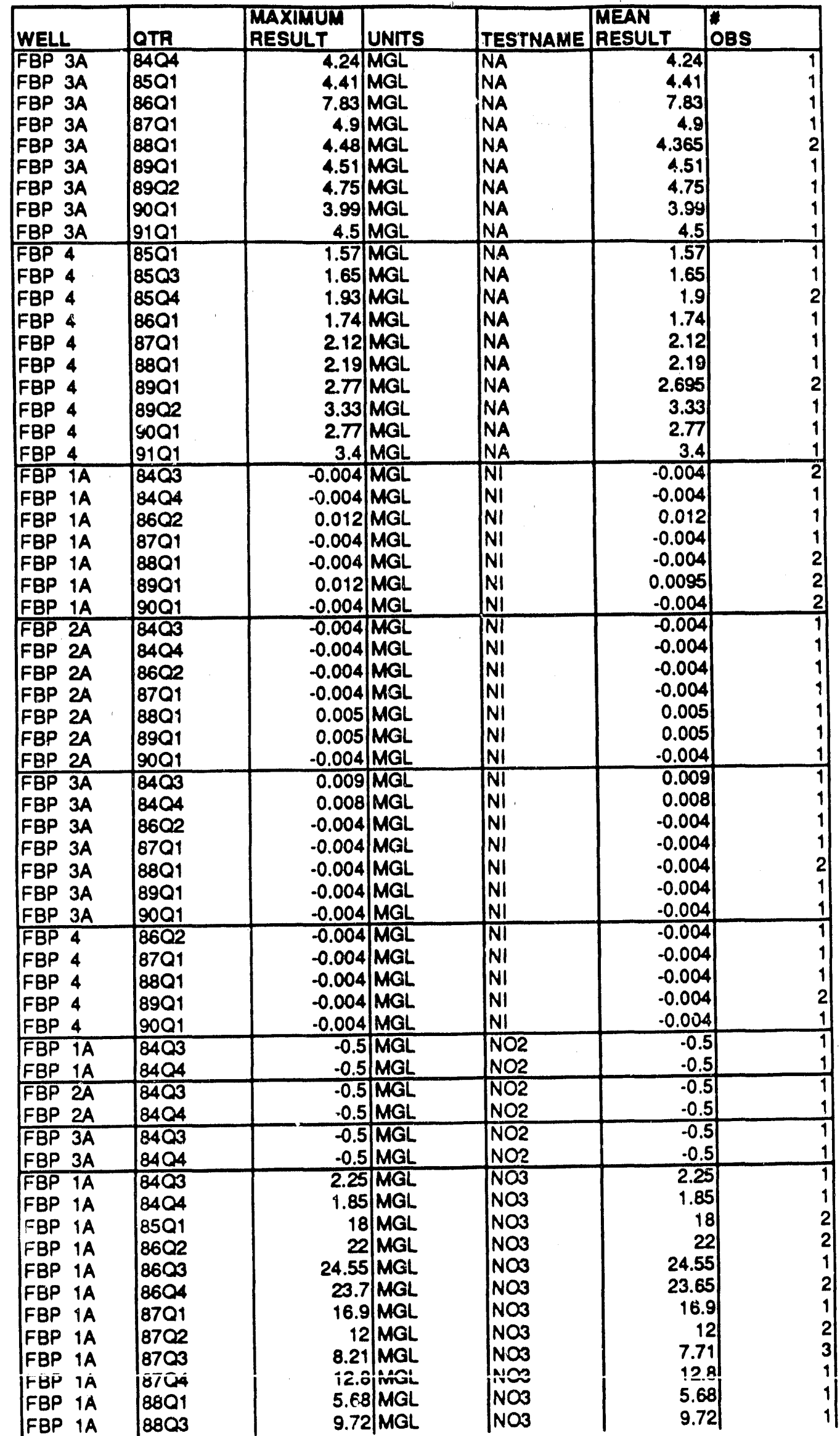

Plot File of Laboratory Analyses at FBP Monitoring Wells - Page 17 
DATA RETPIEVED 7/2/91

\begin{tabular}{|c|c|c|c|c|c|c|}
\hline WELL & atr & $\begin{array}{l}\text { MAXIMUN } \\
\text { RESULT }\end{array}$ & UNITS & TESTNAME & $\begin{array}{l}\text { MEAN } \\
\text { RESULT }\end{array}$ & OBS \\
\hline \begin{tabular}{|ll}
$F B P$ & $1 A$ \\
$F B P$ & $1 A$ \\
$F B P$ & $1 A$ \\
$F B P$ & $1 A$ \\
$F B P$ & $1 A$
\end{tabular} & $\begin{array}{l}8901 \\
8902 \\
8903 \\
9001 \\
9101\end{array}$ & $\begin{array}{r}9.01 \\
9.8 \\
0.38 \\
8.06 \\
6.95\end{array}$ & $\begin{array}{l}\text { MGL } \\
\text { MGL } \\
\text { MGL } \\
\text { MGL } \\
\text { MGL }\end{array}$ & $\begin{array}{l}\mathrm{NO3} \\
\mathrm{NO3} \\
\mathrm{NO3} \\
\mathrm{NO3} \\
\mathrm{NO3}\end{array}$ & $\begin{array}{r}9.01 \\
9.8 \\
0.38 \\
8.03 \\
6.4\end{array}$ & $\begin{array}{l}1 \\
1 \\
1 \\
2 \\
2\end{array}$ \\
\hline FBP 2A & 8403 & 2 & MGL & NO3 & $\begin{array}{r}2 \\
\end{array}$ & 1 \\
\hline $\begin{array}{ll}\text { FBP } & 2 A \\
\text { FBP } & 2 A\end{array}$ & $\begin{array}{l}84 Q 4 \\
8501\end{array}$ & $\begin{array}{r}1.73 \\
1.8\end{array}$ & $\begin{array}{l}\text { MGL } \\
\text { MGL }\end{array}$ & $\begin{array}{l}\mathrm{NO3} \\
\mathrm{NO3}\end{array}$ & $\begin{array}{r}1.73 \\
1.775\end{array}$ & $\begin{array}{l}1 \\
2\end{array}$ \\
\hline FBP 2A & $86 \mathrm{Q} 2$ & 1.58 & MGL & NO3 & 1.58 & 1 \\
\hline $\begin{array}{ll}\text { FBP } & 2 A \\
\text { FBP } & 2 A\end{array}$ & $\begin{array}{l}8603 \\
8604\end{array}$ & 1.29 & MGL & $\begin{array}{l}\mathrm{NO3} \\
\mathrm{NO3}\end{array}$ & 1.29 & 1 \\
\hline $\begin{array}{l}F B P \\
F B P \\
F B A\end{array}$ & $\begin{array}{l}86014 \\
8701\end{array}$ & $\begin{array}{l}1.44 \\
1.27\end{array}$ & $\begin{array}{l}\text { MGL } \\
\text { MGL }\end{array}$ & NO3 & $\begin{array}{l}1.49 \\
1.27\end{array}$ & $\begin{array}{l}1 \\
1\end{array}$ \\
\hline FBP 2A & 8702 & 1.5 & MGL & NO3 & 1.5 & 1 \\
\hline $\begin{array}{l}\text { FBP } 2 A \\
\text { FBP } 2 A\end{array}$ & $\begin{array}{l}8703 \\
8704\end{array}$ & $\begin{array}{l}10.2 \\
3.18\end{array}$ & $\begin{array}{l}\text { MGL } \\
\text { MGL }\end{array}$ & $\begin{array}{l}\mathrm{NO3} \\
\mathrm{NO3}\end{array}$ & $\begin{array}{r}10.2 \\
3.165\end{array}$ & $\begin{array}{l}1 \\
2\end{array}$ \\
\hline FBP $2 A$ & 8801 & 2.19 & MGL & $\mathrm{NO3}$ & 2.19 & 1 \\
\hline FBP 2A & 8803 & 1.81 & $M G L$ & $\mathrm{NO}_{3}$ & 1.81 & 1 \\
\hline $\mid \begin{array}{ll}F B P & 2 A \\
F B P & 2 A\end{array}$ & $\begin{array}{l}8901 \\
8902\end{array}$ & $\begin{array}{r}1.51 \\
12\end{array}$ & $\begin{array}{l}\text { MGL } \\
M G L\end{array}$ & $\begin{array}{l}\mathrm{NO3} \\
\mathrm{NO3}\end{array}$ & $\begin{array}{r}1.51 \\
1.2\end{array}$ & $\begin{array}{l}1 \\
1\end{array}$ \\
\hline FBP $2 A$ & 8903 & 0.78 & MGL & NO3 & 0.78 & 1 \\
\hline FBP 2A & 9001 & 1.49 & MGL & $\mathrm{NO3}$ & 1.49 & 1 \\
\hline FBP 2A & 9101 & 2.27 & MGL & $\mathrm{NO3}$ & 2.27 & 1 \\
\hline FBP 3A & 8403 & -0.5 & MGL & NO3 & -0.5 & 1 \\
\hline FBP $3 A$ & 8404 & -0.5 & MGL & $\mathrm{NO3}$ & -0.5 & 1 \\
\hline FBP $3 A$ & $85 Q 1$ & 0.5 & MGL & $\mathrm{NO3}$ & 0.5 & 1 \\
\hline FBP 3A & 8602 & 0.54 & MGL & $\begin{array}{l}\mathrm{NO3} \\
\mathrm{NO3}\end{array}$ & $\begin{array}{l}0.54 \\
1.08\end{array}$ & $\begin{array}{l}1 \\
1\end{array}$ \\
\hline $\begin{array}{ll}F B P & 3 A \\
F B P & 3 A\end{array}$ & $\begin{array}{l}8603 \\
8604\end{array}$ & $\begin{array}{r}1.08 \\
0.4\end{array}$ & $\begin{array}{l}\text { MGL } \\
\text { MGL }\end{array}$ & $\begin{array}{l}\mathrm{NO3} \\
\mathrm{NO3}\end{array}$ & $\begin{array}{r}1.08 \\
0.4\end{array}$ & 1 \\
\hline FBP $3 A$ & 8701 & 0.31 & MGL & NO3 & 0.31 & 1 \\
\hline FBP 3A & $87 Q 2$ & 0.3 & MGL & $\mathrm{NO3}$ & $\begin{array}{r}0.3 \\
0.67\end{array}$ & 1 \\
\hline $\mid \begin{array}{ll}F B P & 3 A \\
\text { FBP } & 3 A\end{array}$ & $\begin{array}{l}8703 \\
8704\end{array}$ & $\begin{array}{l}0.67 \\
0.58\end{array}$ & $\begin{array}{l}\text { MGL } \\
\text { MGL }\end{array}$ & $\begin{array}{l}\mathrm{NO3} \\
\mathrm{NO3}\end{array}$ & $\begin{array}{l}0.67 \\
0.58\end{array}$ & 1 \\
\hline FBP $3 A$ & 8801 & 0.5 & $M G L$ & NO3 & 0.5 & 1 \\
\hline FBP $3 A$ & 8803 & 0.44 & MGL & $\mathrm{NO3}$ & 0.44 & 1 \\
\hline FBP $3 A$ & 8901 & 0.291 & MGL & $\begin{array}{l}\mathrm{NO3} \\
\mathrm{NO3}\end{array}$ & $\begin{array}{r}0.291 \\
0.34\end{array}$ & $\begin{array}{l}1 \\
1\end{array}$ \\
\hline $\begin{array}{ll}\text { FBP } & 3 A \\
\text { FBP } & 3 A\end{array}$ & $\begin{array}{l}89022 \\
8903\end{array}$ & $\begin{array}{l}0.34 \\
0.29\end{array}$ & $\begin{array}{l}\text { MGL } \\
\text { MGL }\end{array}$ & $\begin{array}{l}\mathrm{NO3} \\
\mathrm{NO3}\end{array}$ & 0.28 & 2 \\
\hline FBP $3 A$ & 2001 & 0.18 & MGL & NO3 & 0.18 & 1 \\
\hline FBP $3 A$ & 9101 & 0.26 & MGL & $\mathrm{NO3}$ & 0.26 & 1 \\
\hline$\overline{F B P ~} 4$ & 8501 & -0.5 & MGL & NO3 & .0 .5 & 1 \\
\hline FBP 4 & 8503 & -0.5 & MGL & NO3 & -0.5 & 1 \\
\hline FBP 4 & 8504 & -0.5 & MGL & $\mathrm{NO3}$ & -0.5 & 2 \\
\hline FBP 4 & 8601 & 0.62 & MGL & $\mathrm{NO3}$ & 0.62 & 1 \\
\hline FBP 4 & $86 Q 2$ & 0.5 & MGL & $\mathrm{NO3}$ & 0.5 & 1 \\
\hline FBP 4 & 8603 & 0.3 & MGL & $\mathrm{NO3}$ & 0.3 & 1 \\
\hline FBP 4 & $86 \bigcirc 4$ & 0.43 & MGL & $\mathrm{NO3}$ & 0.43 & 1 \\
\hline FBP 4 & 8701 & 0.44 & MGL & NO3 & 0.44 & 1 \\
\hline FBP 4 & $\begin{array}{l}87 Q 2 \\
8703\end{array}$ & $\begin{array}{l}0.45 \\
0.76\end{array}$ & $\begin{array}{l}\text { MGL } \\
\text { MGL }\end{array}$ & $\begin{array}{l}\mathrm{NO3} \\
\mathrm{NO3}\end{array}$ & $\begin{array}{l}0.45 \\
0.76\end{array}$ & $\begin{array}{l}1 \\
1\end{array}$ \\
\hline $\begin{array}{ll}\text { FBP } & 4 \\
\text { FBP } & 4\end{array}$ & $\begin{array}{l}8703 \\
8704\end{array}$ & $\begin{array}{l}0.76 \\
0.68\end{array}$ & MGL & NO3 & 0.68 & 1 \\
\hline FBP 4 & 8801 & 0.58 & MGL & NO3 & 0.58 & 1 \\
\hline FBP 4 & 8803 & $\begin{array}{r}0.47 \\
\end{array}$ & MGL & $\begin{array}{l}\mathrm{NO3} \\
\mathrm{NO3}\end{array}$ & $\begin{array}{r}0.47 \\
0.377\end{array}$ & 1 \\
\hline FBP 4 & 8901 & $\begin{array}{r}0.377 \\
0.48\end{array}$ & MGL & $\begin{array}{l}\mathrm{NO3} \\
\mathrm{NO} 3\end{array}$ & $\begin{array}{r}0.377 \\
0.48\end{array}$ & 1 \\
\hline FBP 4 & 8902 & $\begin{array}{l}0.48 \\
0.43\end{array}$ & MGL & $\begin{array}{l}\mathrm{NO3} \\
\mathrm{NO3}\end{array}$ & $\begin{array}{l}0.48 \\
0.43\end{array}$ & 1 \\
\hline FBP 4 & 8903 & $\begin{array}{l}0.43 \\
0.26\end{array}$ & MGL & $\begin{array}{l}\mathrm{NO3} \\
\mathrm{NO3}\end{array}$ & $\begin{array}{l}0.43 \\
0.26\end{array}$ & 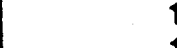 \\
\hline $\mid \begin{array}{ll}\text { FBP } & 4 \\
\text { FBP } & 4\end{array}$ & $\begin{array}{l}9001 \\
9101\end{array}$ & $\begin{array}{l}0.26 \\
0.31\end{array}$ & $\begin{array}{l}\text { MGL } \\
\text { MGL }\end{array}$ & $\begin{array}{l}\mathrm{NO3} \\
\mathrm{NO3}\end{array}$ & $\begin{array}{l}0.26 \\
0.31\end{array}$ & 1 \\
\hline FBP 4 & $\frac{91 Q 1}{8403}$ & $\begin{array}{r}0.31 \\
4\end{array}$ & $\frac{M G L}{\text { THON }}$ & ODOR & 4 & $\frac{1}{1}$ \\
\hline FBP IA & 8403 & $\begin{array}{l}4 \\
0\end{array}$ & & ODOR & $\begin{array}{l}4 \\
0\end{array}$ & 1 \\
\hline FBP $1 A$ & 8404 & $\frac{0}{4}$ & THON & & 의 & 1 \\
\hline FBP 2A & 8403 & 4 & THON & ODOA & 4 & 1 \\
\hline FBP 2A & 8404 & 0 & THON & ODOR & of & 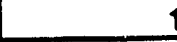 \\
\hline FBP $3 \AA$ & 8403 & $\overline{2}$ & ITUÓN & UUŪ & ह & 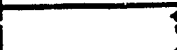 \\
\hline FBP $3 A$ & 8404 & 0 & THON & LOOR & 이 & \\
\hline
\end{tabular}

Plot File of Laboratory Analyses at FBP Monitoring Wells - Page 18 
DATA RETRIEVED 7/2/91

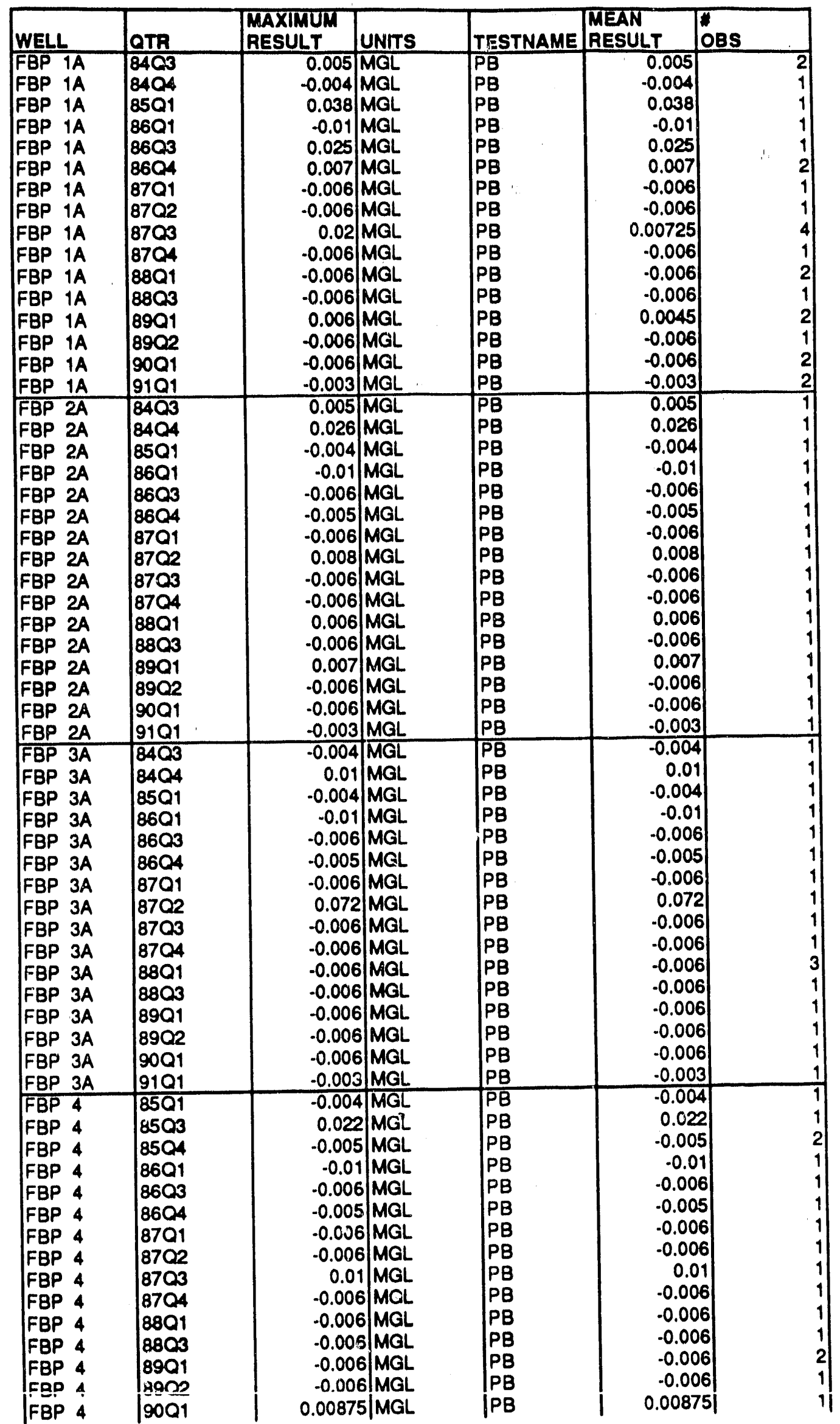

Plot File of Laboratory Analyses at FBP Monitoring Wells - Page 19 
DATA RETRIEVED 7/2/91

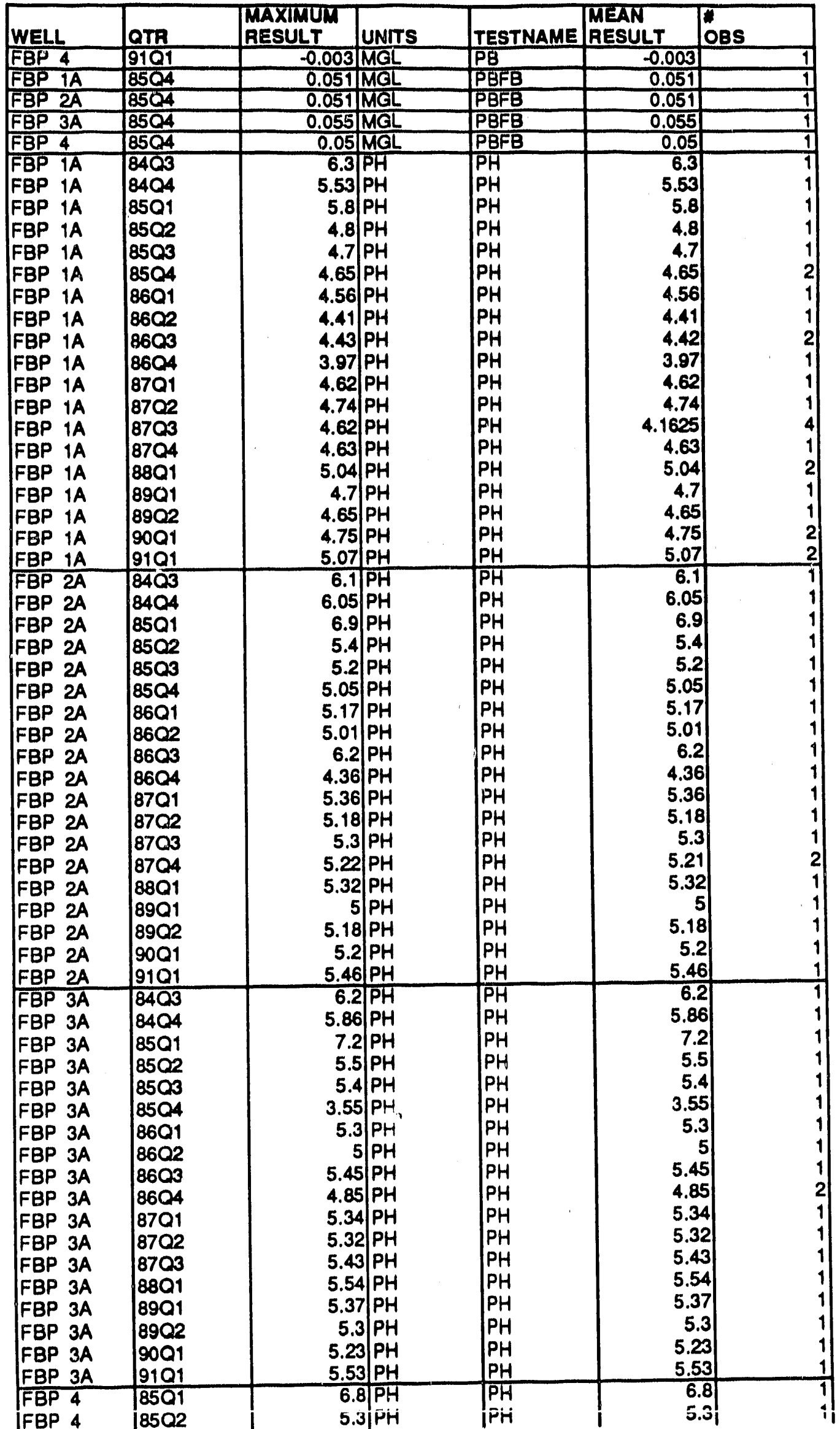

Plot File of Laboratory Analyses at FBP Monitoring Wells - Page 20 
DATA RETRIEVED 7/2/91

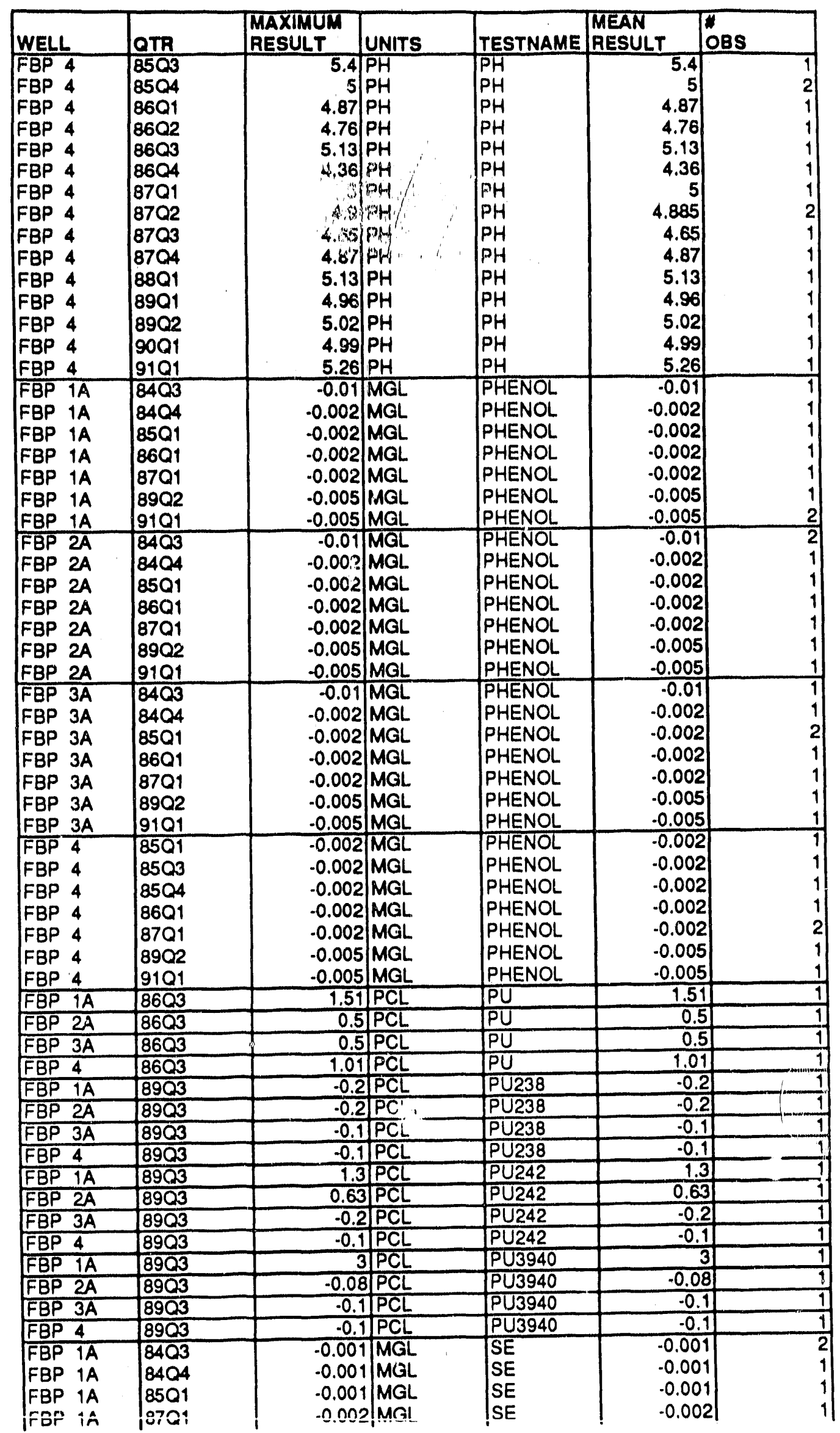

Plot File of Laboratoly Analyses at FBP Monitoring Wells - Page 21 
DATA RETRIEVED 7/2/91

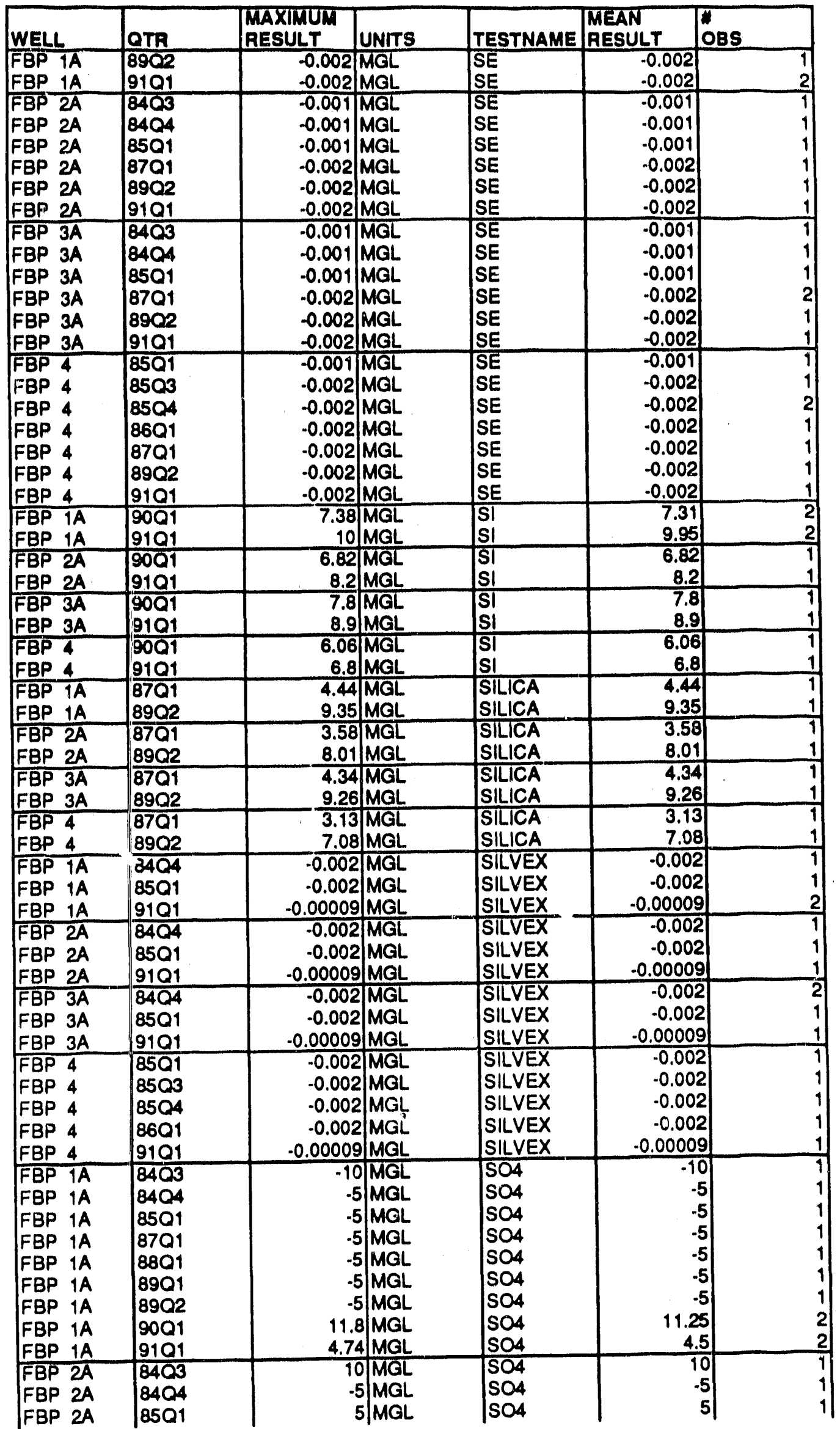

Plot File of Laboratory Analyses at FBP Monitoring Wells - Page 22 
DATA RETRIEVED 7/2/91

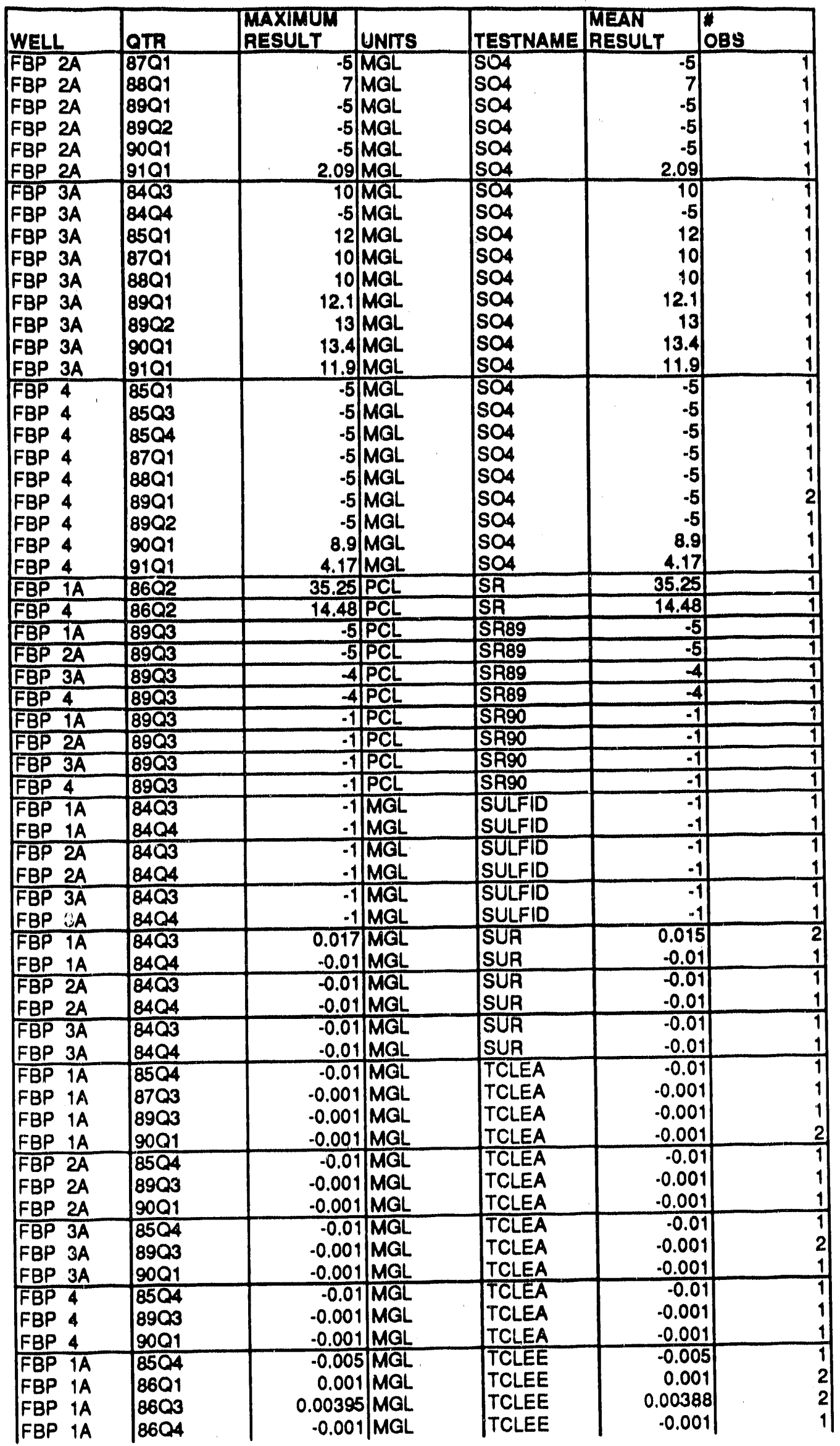

Plot File of Laboratory Analyses at $F B \bar{P}$ Monitoring Viviis - Fage $2 \bar{S}$ 
DATA RETRIEVED 7/2/91

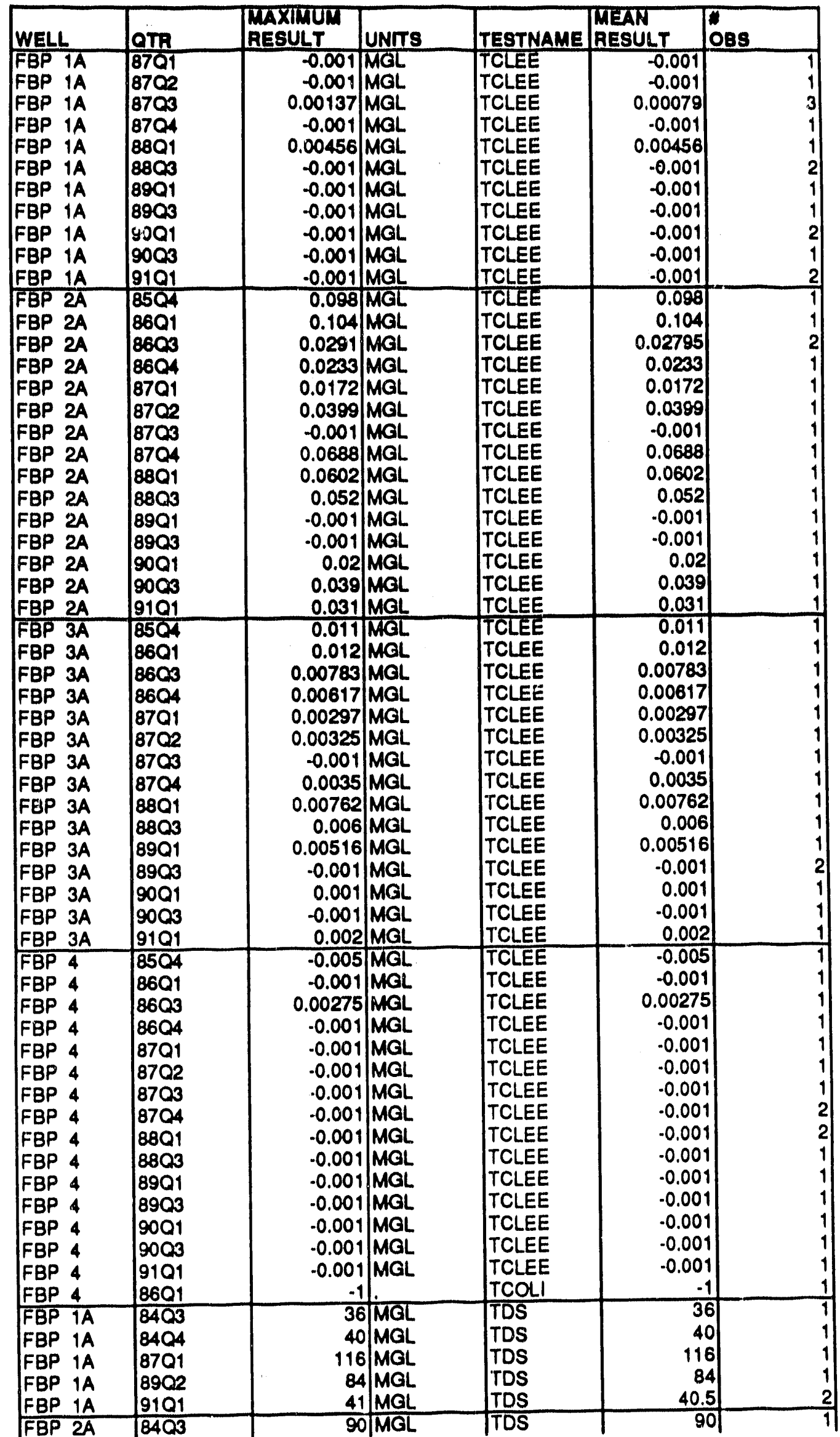

Plot File of Laboratory Analyses at FBP Monitoring Wells - Page 24 
DATA RETRIEVED 7/2/91

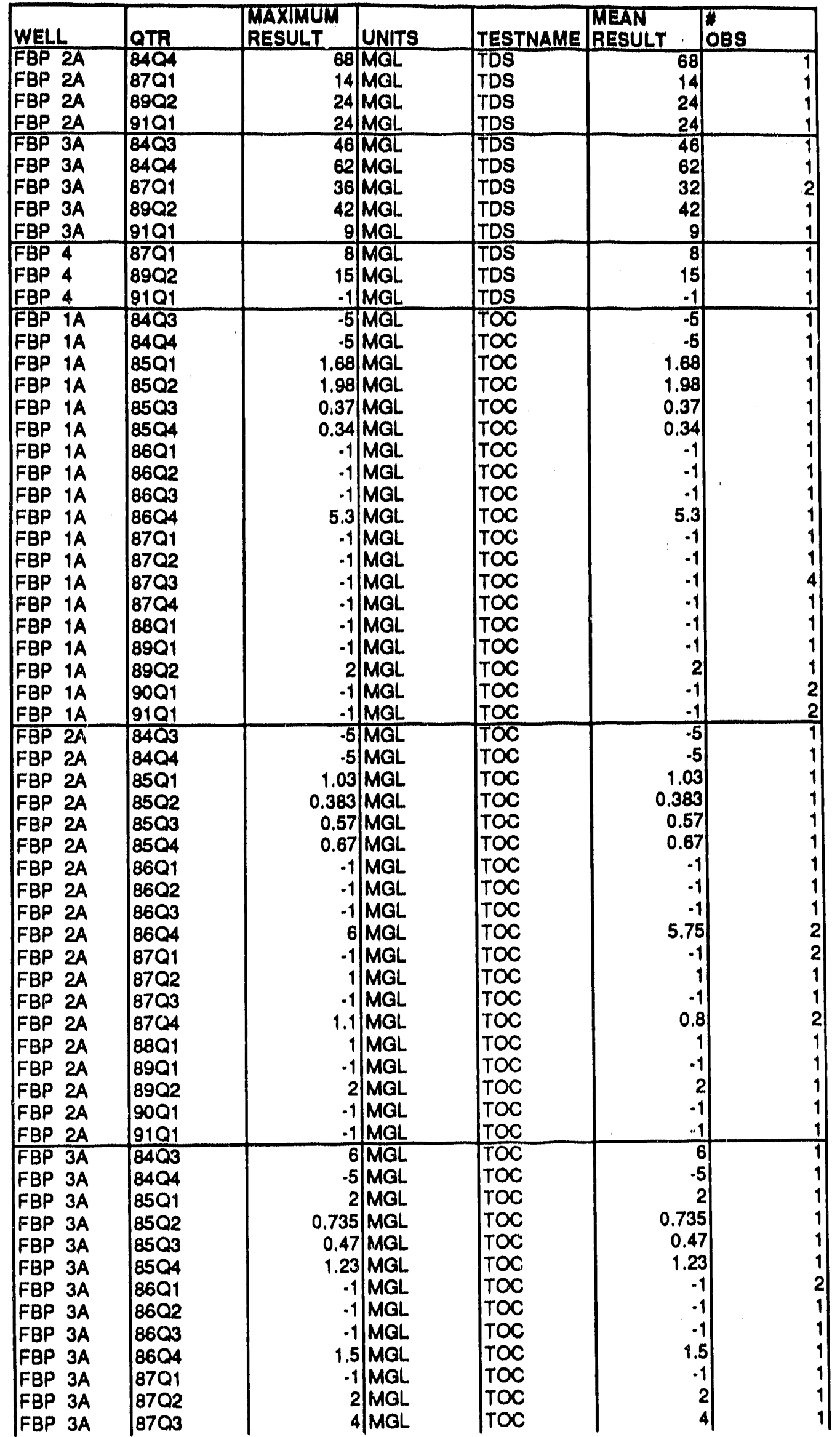

Plot File of Laboratory Analyses at FBP Monitoring Wells - Page 25 
DATA RETRIEVED 7/2/91

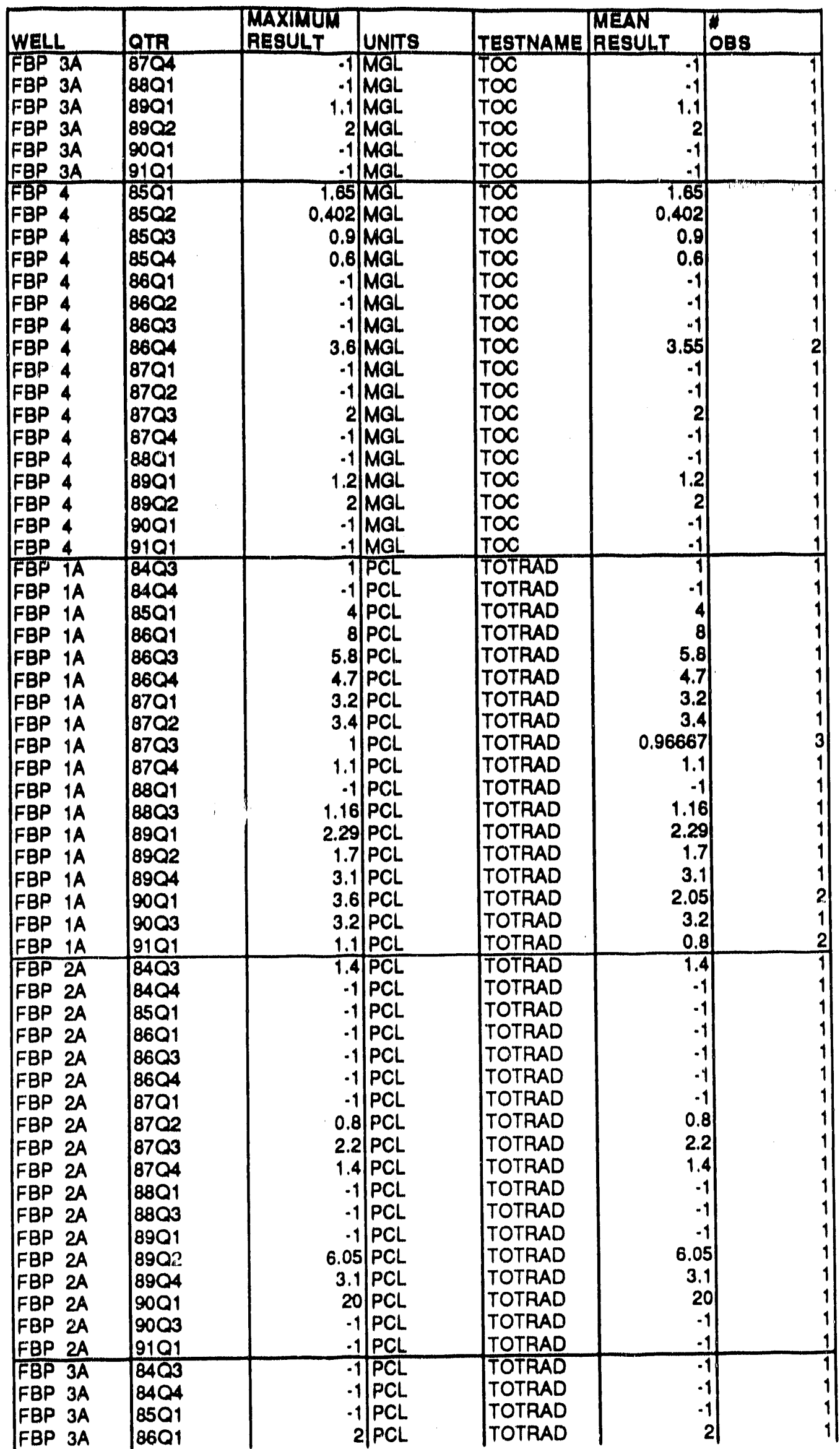

Plot File of Laboratory Analyses at FBP Montoring Wolls - Page 26 
DATA RETRIEVED 7/2/91

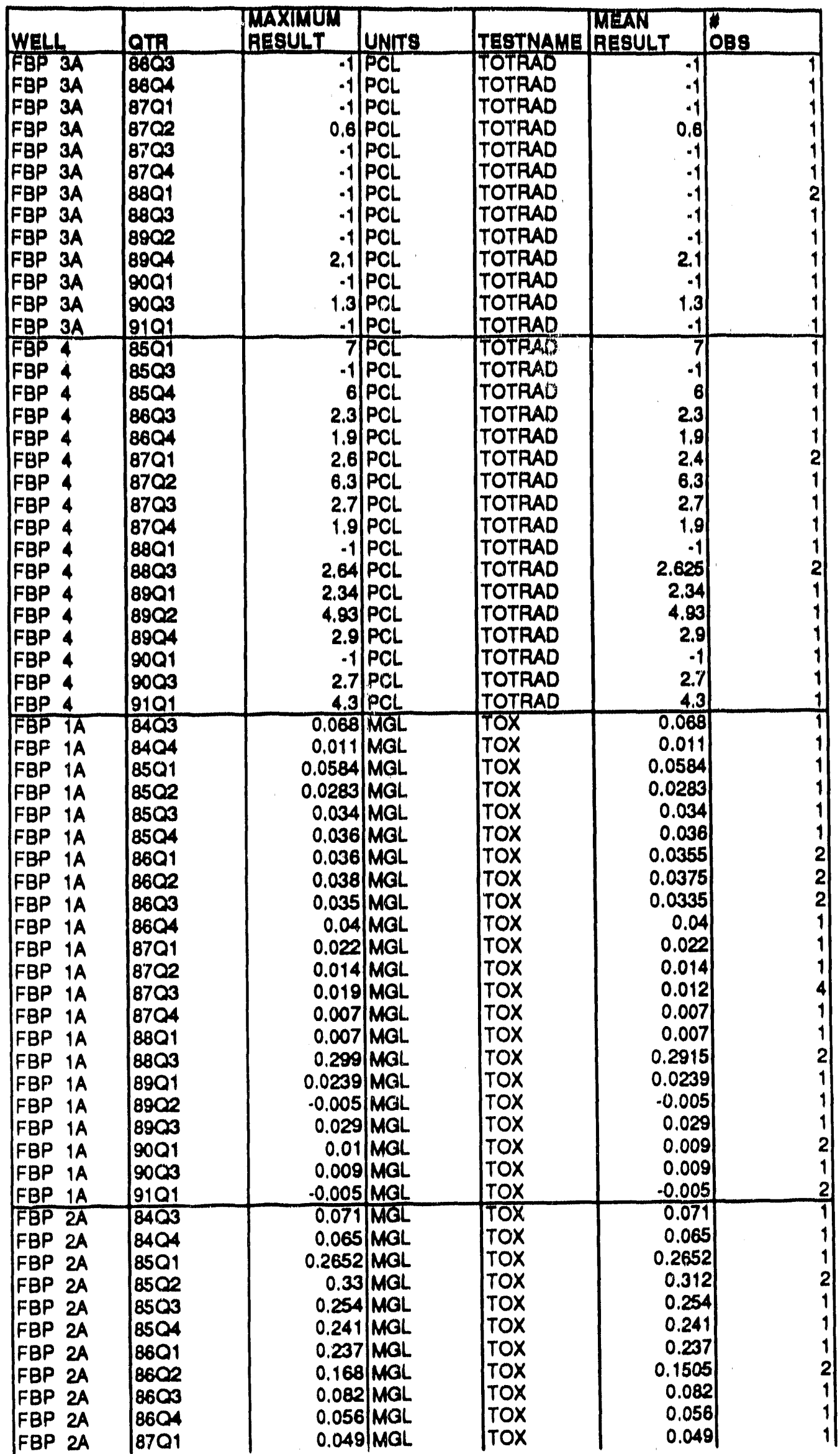

Plot File of Laboraiory Analysos at FBP Monitoring Wells - Page 27 
DATA RETAIEVED 7/2/21

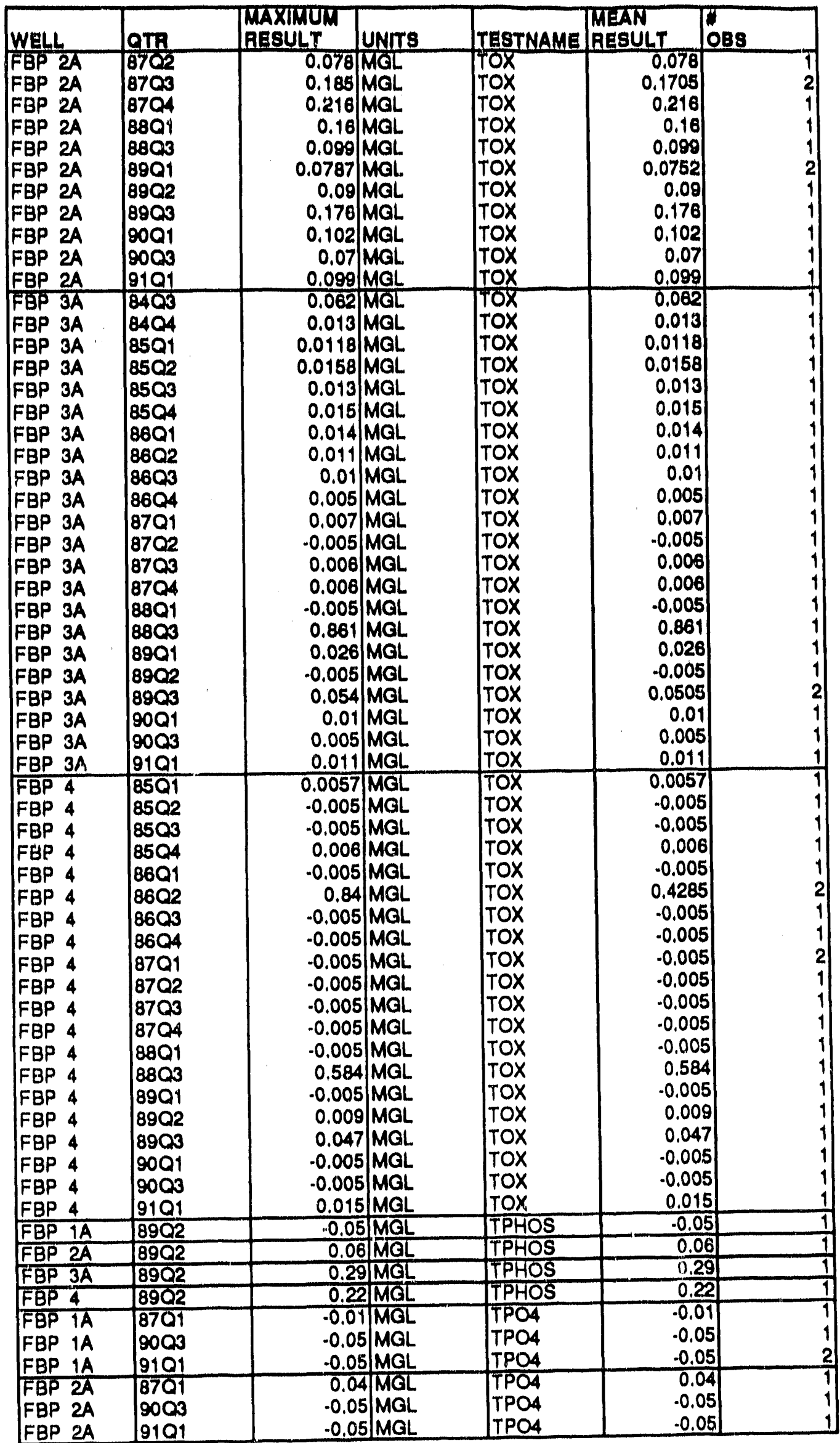

Plot File of Laboratory Analyses at FBP Monitoring Wells - Page 28 
DATA RETAIEVED 7/2/91

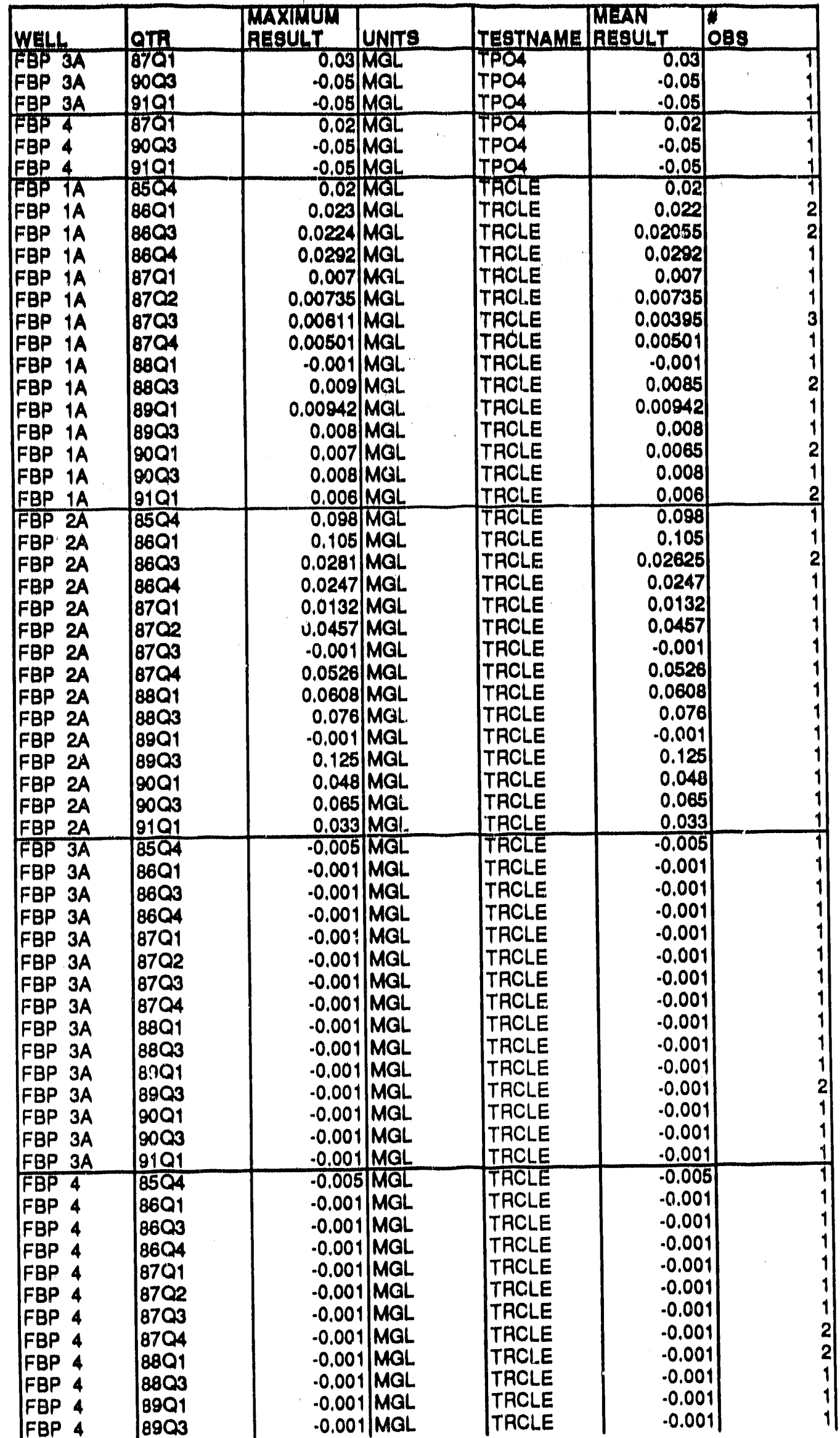

Plot File of Laboratory Analyses at FBP Monitoring Wells - Page 29 
DATA RETRIEVED 7/2/91

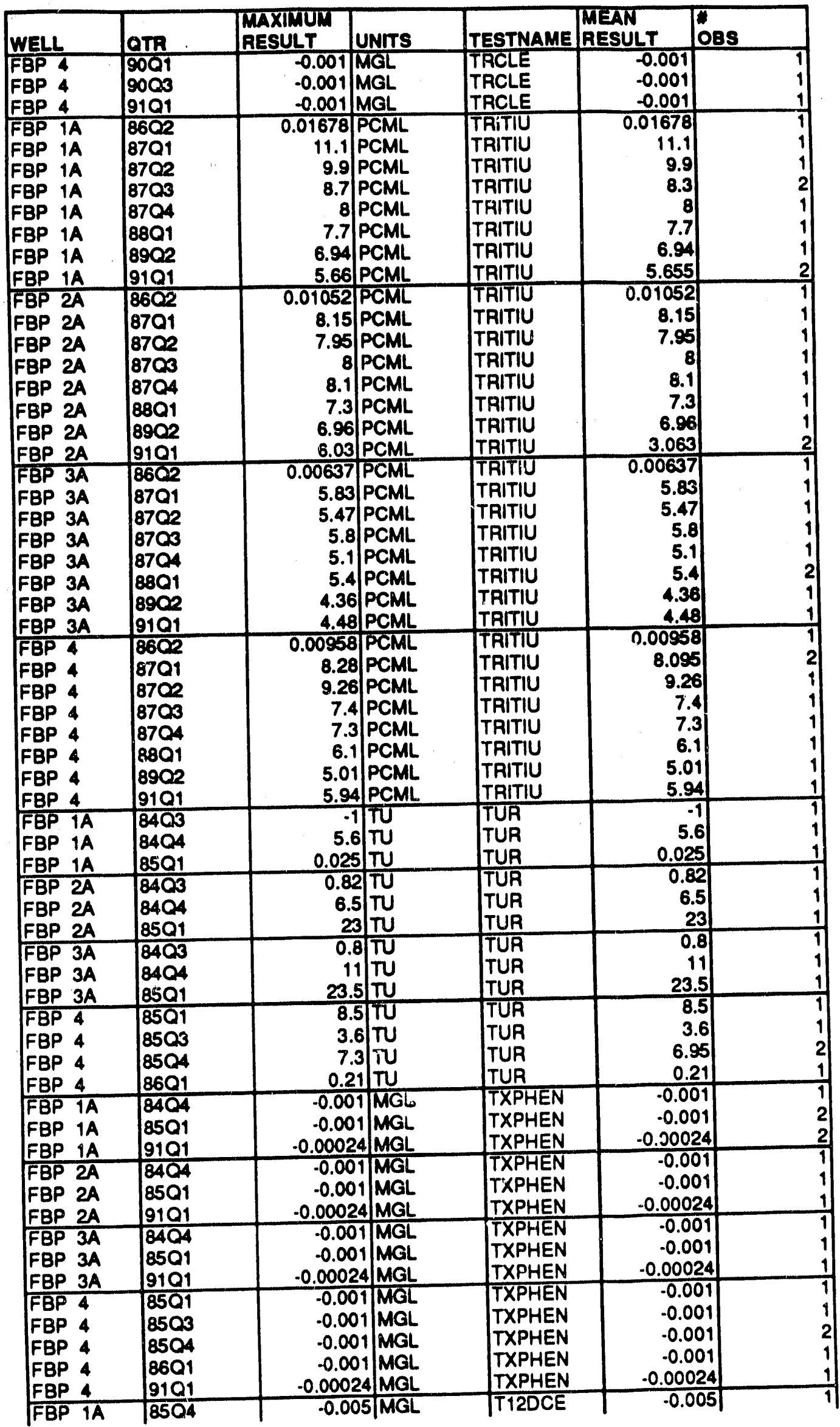

Plot File of Laboratory Analyses at FBP Monitoring Wolls - Page 3 ? 
DATA RETRIEVED 7/2/91

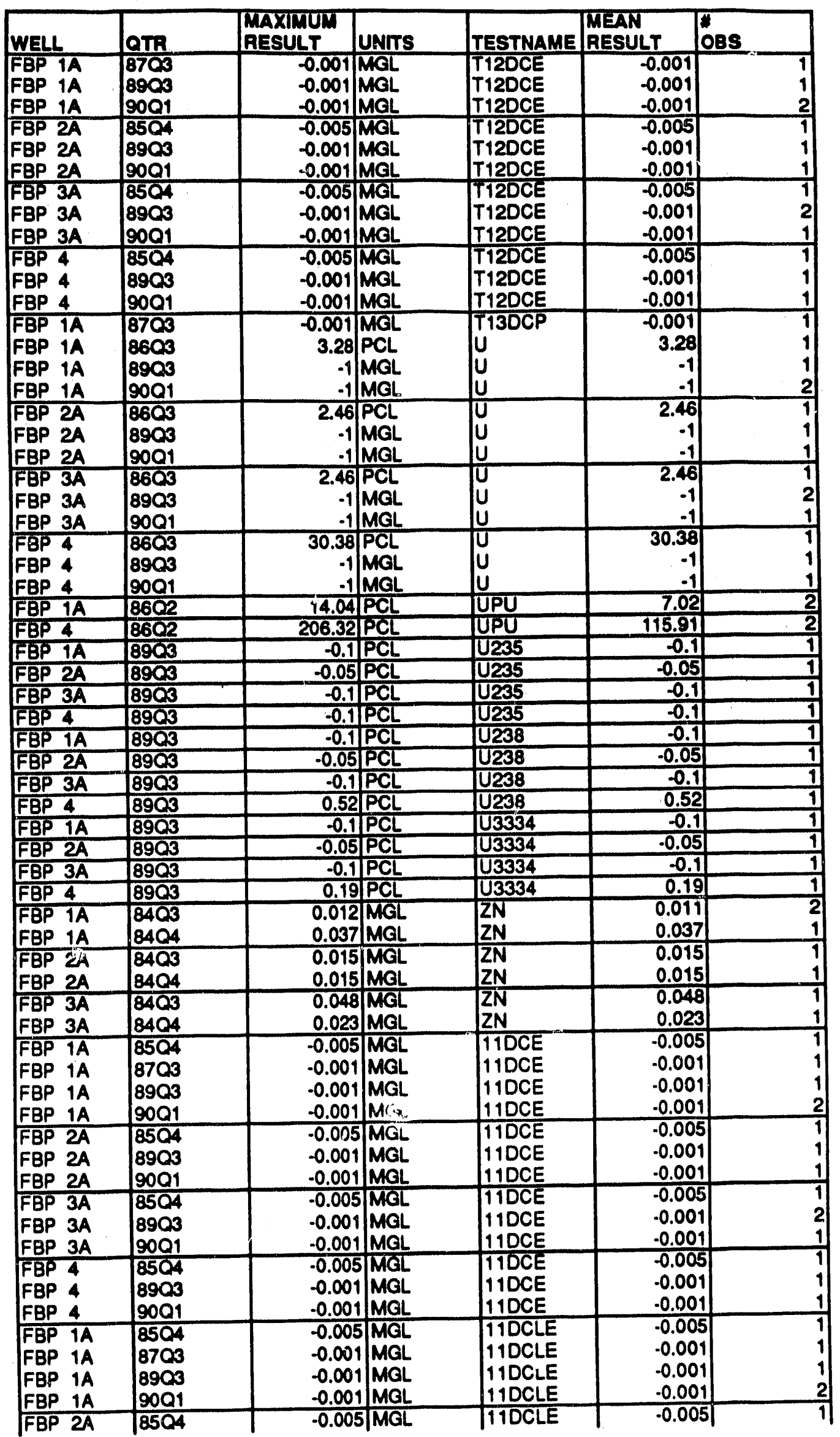

Plot File of Laboratory Analyses at FBP Monitoring Wolls - Page 31 
DATA RETRIEVED 7/2/91

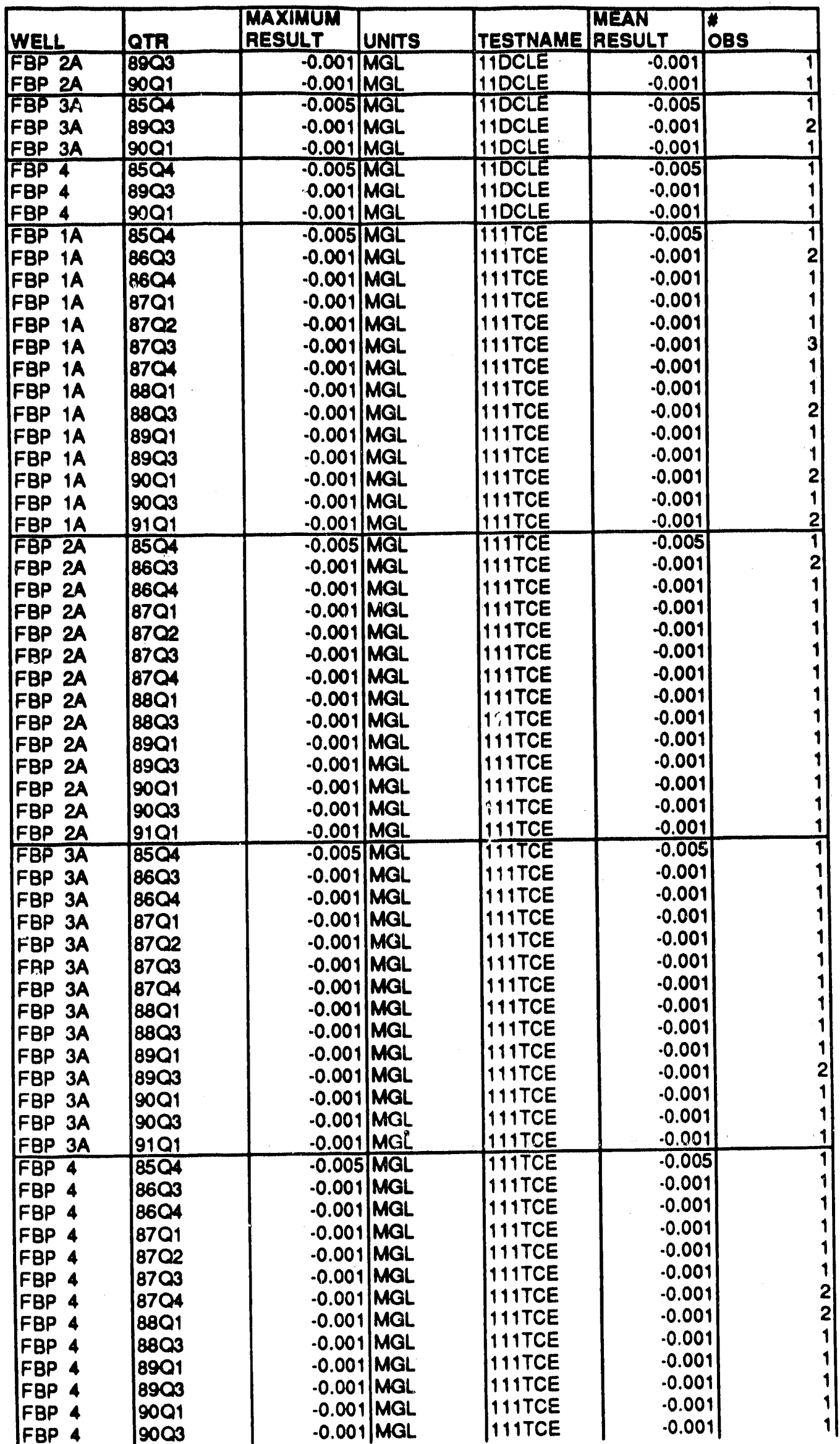


DATA RETRIEVED 7/2/91

\begin{tabular}{|c|c|c|c|c|c|c|}
\hline WELL & QTR & \begin{tabular}{|l|} 
MAXINUN \\
RESULT
\end{tabular} & UNITS & ITESTNAME & $\begin{array}{l}\text { MEAN } \\
\text { RESULT }\end{array}$ & OBS \\
\hline FBP 4 & 9101 & -0.001 & MGL & 111TCE & .0 .001 & 1 \\
\hline FBP 1A & $850 !$ & -0.005 & MGL & 112TCE & -0.005 & 1 \\
\hline FBP $1 A$ & 8703 & -0.001 & MGL & 1 12TCE & -0.001 & 1 \\
\hline $\begin{array}{ll}F B P & 1 A \\
\text { FBP } & 1 A\end{array}$ & $\begin{array}{l}8903 \\
9001\end{array}$ & $\begin{array}{l}-0.001 \\
-0.001\end{array} \mid$ & $\begin{array}{l}\text { MGL } \\
\text { MGL }\end{array}$ & $\begin{array}{l}\text { 112TCE } \\
112 \text { TCE }\end{array}$ & $\begin{array}{l}-0.001 \\
-0.001\end{array}$ & $\begin{array}{l}1 \\
2\end{array}$ \\
\hline FBP 2A & 8504 & -0.005 & MGL & 112TCE & .0 .005 & 1 \\
\hline$F B P 2 A$ & 8903 & -0.001 & MGL & 112TCE & -0.001 & 1 \\
\hline$F B P 2 A$ & 19001 & $-0.001]$ & MGL & 112TCE & -0.001 & 1 \\
\hline FBP $3 A$ & 8504 & -0.005 & MGL & 112TCE & -0.005 & 1 \\
\hline FBP $3 A$ & 8903 & -0.001 & MGL & 112TCE & -0.001 & 2 \\
\hline FBP $3 A$ & 9001 & -0.001 & MGL & 112TCE & -0.001 & 1 \\
\hline FBP 4 & 8504 & .0 .005 & MGL & 112TCE & .0 .005 & 1 \\
\hline FBP 4 & 8903 & -0.001 & MGL & 112TCE & -0.001 & 1 \\
\hline FBP 4 & 9001 & -0.001 & MGL & \& 12TCE & -0.001 & 1 \\
\hline FBP IA & 8504 & 0.045 & MGL & $120 C D 4$ & 0.045 & 1 \\
\hline FBP 2A & 8504 & 0.051 & MGL & $120 \mathrm{CD4}$ & 0.051 & 1 \\
\hline FBP $3 A$ & 8504 & 0.05 & MGL & $120 \mathrm{CD4}$ & 0.05 & 1 \\
\hline FBP 4 & 8504 & 0.052 & MGL & $120 \mathrm{CD4}$ & 0.052 & 1 \\
\hline FBP 1A & 5504 & .0 .001 & MGL & 12DCLE & -0.001 & 1 \\
\hline FBP $1 A$ & 8703 & -0.001 & MGL & 12DCLE & -0.001 & 1 \\
\hline FBP $1 A$ & 8903 & -0.001 & MGL & 12DCLE & -0.001 & 1 \\
\hline IFBP IA & 9001 & -0.001 & MGL & 12DCLE & -0.001 & 2 \\
\hline FBP 2A & 8504 & -0.001 & MGL & 12DCLE & -0.001 & 1 \\
\hline FBP 2A & 8903 & -0.001 & MGL & 12DCLE & -0.001 & 1 \\
\hline FBP 2A & 9001 & .0 .001 & $M G L$ & 12DCLE & -0.001 & 1 \\
\hline FBP $3 A$ & 8504 & .0 .001 & MGL & 120CLE & .0 .001 & 1 \\
\hline FBP $3 A$ & 8903 & -0.001 & MGL & 12DCLE & -0.001 & 2 \\
\hline FBP $3 A$ & 9001 & -0.001 & MGL & 12DCLE & -0.001 & 1 \\
\hline FBP 4 & 8504 & -0.001 & MGL & 12DCLE & -0.001 & 1 \\
\hline FBP 4 & 8903 & -0.001 & MGL & 12DCLE & -0.001 & 1 \\
\hline FBP 4 & 9001 & -0.001 & MGL & 12DCLE & -0.001 & 1 \\
\hline FBP IA & 8504 & -0.01 & MGL & 12DCLP & -0.01 & 1 \\
\hline FBP 1A & 8703 & -0.001 & MGL & 12DCLP & -0.001 & 1 \\
\hline FBP $1 A$ & 8903 & -0.001 & MGL & 12DCLP & -0.001 & 1 \\
\hline FBP IA & $90 Q 1$ & -0.001 & MGL & 12DCLP & -0.001 & 2 \\
\hline FBP 2A & 2504 & -0.01 & MGL & $12 D C L P$ & -0.01 & 1 \\
\hline FBP $2 A$ & 8903 & -0.001 & MGL & 12DCLP & -0.001 & 1 \\
\hline FBP $2 A$ & 9001 & -0.001 & MGL & $12 D C L P$ & -0.001 & 1 \\
\hline FBP $3 A$ & 8504 & .0 .01 & MGL & $12 D C L P$ & -0.01 & 1 \\
\hline FBP $3 A$ & 8903 & -0.001 & MGL & 12DCLP & -0.001 & 2 \\
\hline FBP $3 A$ & 9001 & -0.001 & MGL & $12 D C L P$ & -0.001 & 1 \\
\hline FBP 4 & 8504 & -0.01 & MGL & 12 DCLP & -0.01 & 1 \\
\hline FBP 4 & 8903 & -0.001 & MGL & 12DCLP & -0.001 & 1 \\
\hline FBP 4 & 9001 & -0.001 & MGL & 12DCLP & -0.001 & 1 \\
\hline FBP 1A & 8504 & -0.005 & MGL & $130 \mathrm{CP}$ & -0.005 & 1 \\
\hline FBP 2A & 8504 & 0.005 & MGL & $\frac{13 D C P}{13 D C}$ & .0 .005 & $\frac{1}{1}$ \\
\hline FBP $3 A$ & 8504 & -10.005 & MGL & $\frac{130}{13 D C P}$ & .0 .005 & $\frac{1}{1}$ \\
\hline FBP 4 & 8504 & -0.005 & MGL & $\frac{130 C P}{130}$ & .0 .005 & $\frac{1}{1}$ \\
\hline FBP $1 A$ & 89023 & -0.001 & MGL & $13 D C P C$ & $\frac{0.001}{.0 .001}$ & $\frac{1}{1}$ \\
\hline FBP $1 A$ & 9001 & -0.001 & MGL & 13DCPC & -0.001 & $\begin{array}{l}1 \\
2 \\
\end{array}$ \\
\hline FBP 2A & 8903 & -0.001 & MGL & 13DCPC & -0.001 & $\frac{5}{1}$ \\
\hline FBP 2A & 9001 & -0.001 & IMGL & $13 D C P C$ & .0 .001 & 1 \\
\hline FBP $3 A$ & 8903 & .0 .001 & MGL & 13DCPC & -0.001 & $\frac{1}{2}$ \\
\hline FBP $3 A$ & 9001 & -0.001 & MGL & 13DCPC & -0.001 & 1 \\
\hline FBP 4 & 8903 & -0.001 & MGL & 13DCPC & -0.001 & 1 \\
\hline FBP 4 & 19001 & -0.001 & MGL & 13DCPC & -0.001 & 1 \\
\hline $\begin{array}{ll}\text { FBP } & 1 \mathrm{~A}\end{array}$ & 8903 & -0.001 & MGL & 130CPT & .0 .001 & 1 \\
\hline FBP $1 A$ & 9001 & -0.001 & MGL & 13DCPT & -0.001 & 2 \\
\hline FBP 2A & 8903 & -0.001 & MGL & 13DCPT & .0 .001 & 1 \\
\hline FEP 2A & 9001 & -0.001 & MGL & 130CPT & -0.001 & 1 \\
\hline FBP $3 A$ & 8903 & -0.001 & MGL & 130CPT & -0.001 & 2 \\
\hline FBP $3 A$ & 9001 & -0.001 & MGL & 13DCPT & -0.001 & 1 \\
\hline FBP 4 & 8903 & .0 .001 & MGL & 13DCPT & -0.001 & 1 \\
\hline
\end{tabular}

Plot File of Laboratory Analyses at FBP Monitoring Wells - Page 33 
DATA RETRIEVED 7/2/91

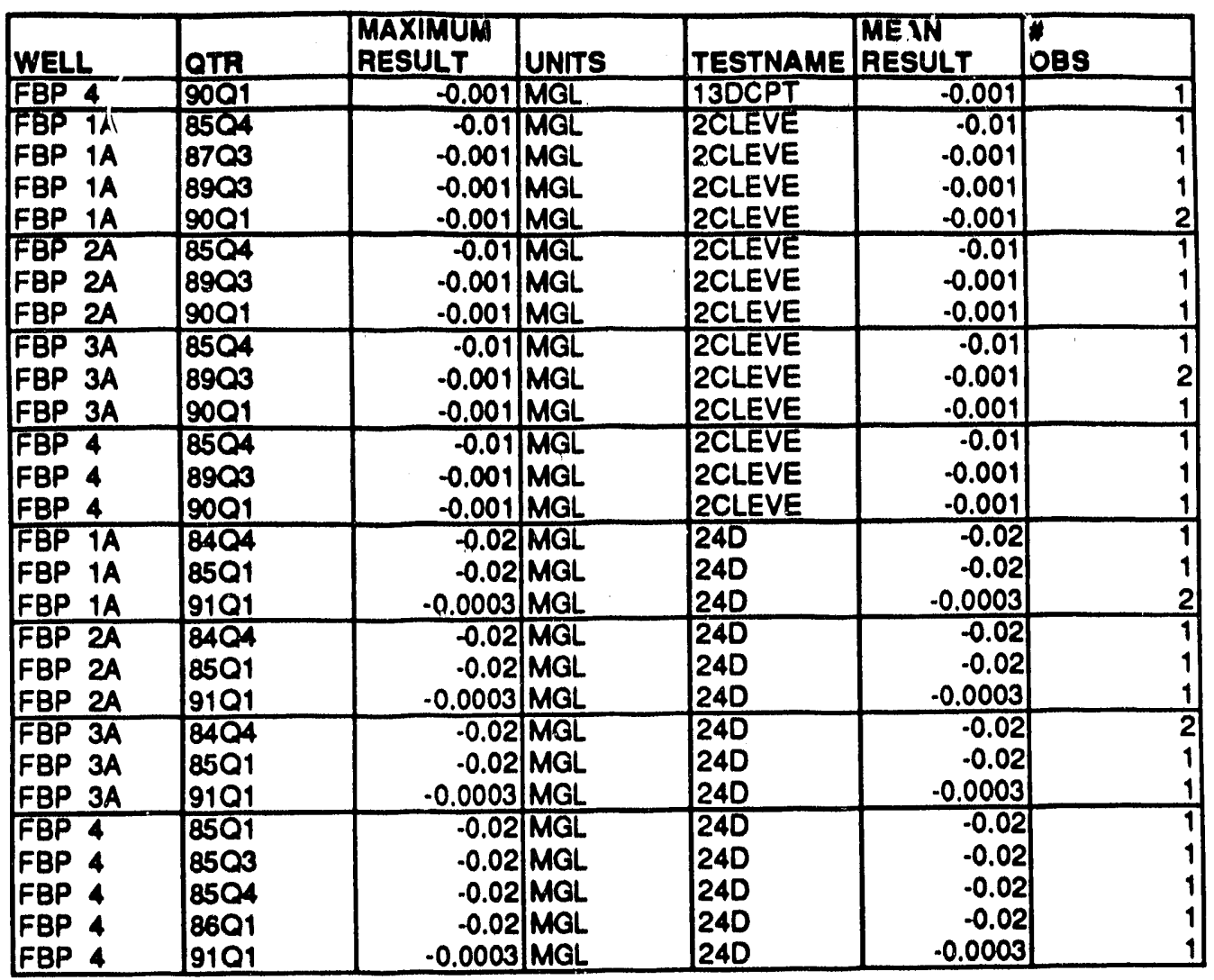


APPENDIX C

FIELD NOTES

AND

GEOLOGIC LOGS 
1

$3 / 12 / 91$

E-Area Railnoad Crosstie Site

Technical Orensight: Charlesh. Travis, $P E, P G ;$

Sanpling Assistantí Sean Assuith

Compaty: Sinnine Envirenmento / Consultants

Sampling Stotien: FRT-1-HP, soil bering, splitspeensamples. SRS Coordinatesicireliminary)$N 79,777,44 ; E 50,408.41$

Preliminary Elevation: 284,2

Beference Paint i Gravod Surface; Pitch/dintroad": station Estabished 3/12/91

Sample In formation:

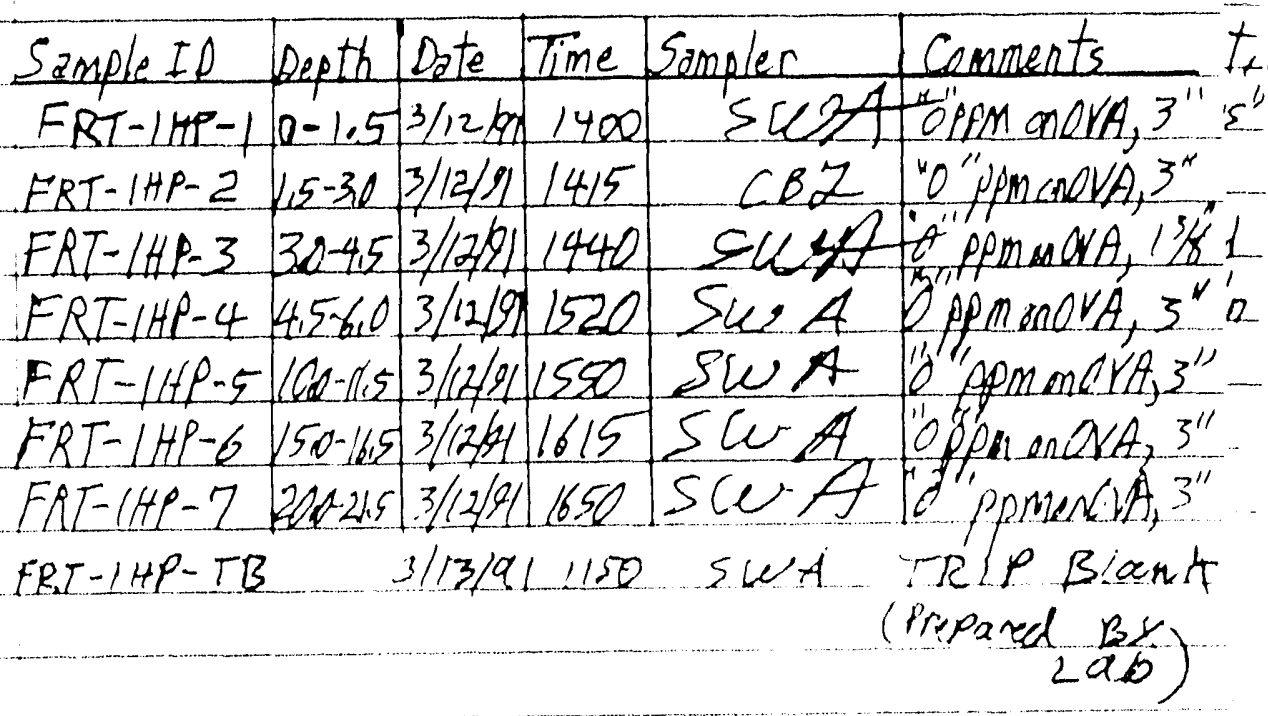

M 


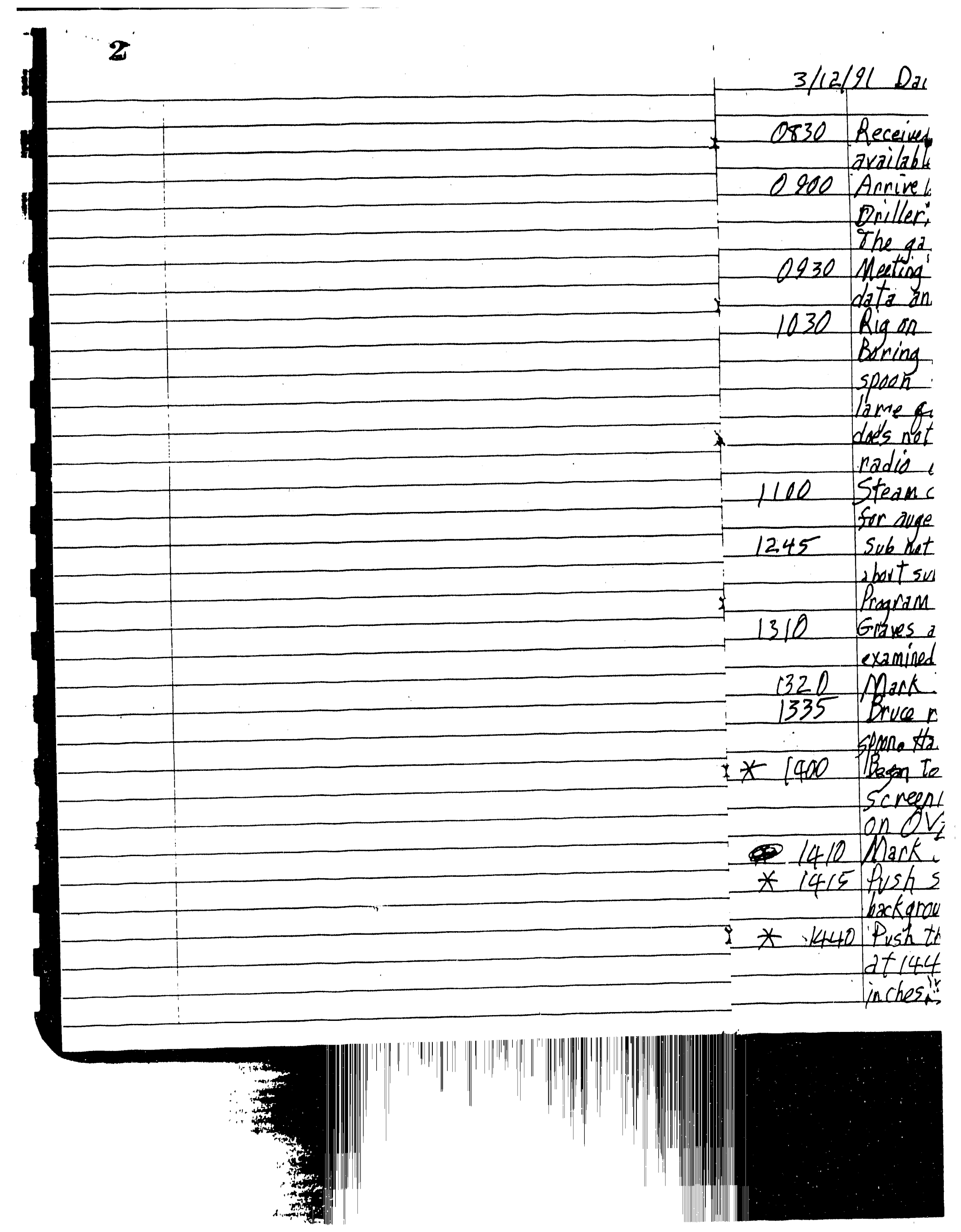


3/12/2l Daily Activities Report

O830 Received Telecom from Dan Wells that drill rig is available, proceed to site.

900 Arrive Well Bldg y Begin. I assembling sampling gear. Driller, James Smith, steam cleaning augers out side The gate to FAred Crosstie site.

0930 Meeting with Janelle Jansen, re: format for Sample data and Daily Activities, etc.

1030 Rigor location of first sampling station, sail Boring FRT-1-HP. We plan to we 3 inch split span for near-sunface soil samples because of large quantity of sample nequinal, but James does net have a sub to adapt te 3 inch spoon. I radio Dan Wells - he said Carl will bring one out Hel Steam cleaner on site, boom up, setting up horses" fer augers.

1245 Sub hat yet en site; contacted Janelle Jensen by radical. Asked. abort substituting hand auger for 3 inch splitspen-not in Program Plan. She contacted Graves, Bruce Kristanion of 1310 Graves arrived site with sample catchers ot 1310. examined the split spoon -dpt site te get sub. 1320 Mark Tones, wo re, visits site 1320

1335 Bruce returns site 1335 with sub for inch spelt spine that to file threads to get it to make up

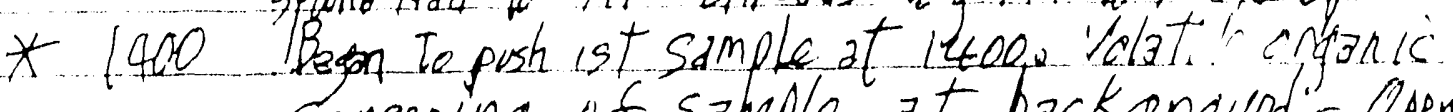
screening of sample at background - Opp n

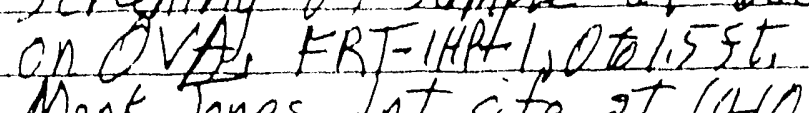

1410 Mark Jones dpt site at 1410

* 1415 Push second sample at 1415 ; OVA rezdingot background. Samples la 2 with 3 "splitsperts.

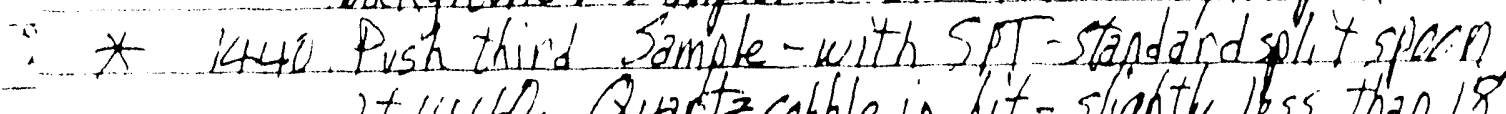
at 1440 Q Quertzceble in rit -slightly thess than 18 inchesinsamphe, sample volume very small with 
4

$3 / 12 / 91$

inch split spoon (13/8" ID); "unable to fill bottles from labe Elected To use 3 " split spoon on remaining samples. This is moke time con suing, especially withlonly one drive head and it requiring wrenches because of bad threads. Also econ wrenches each time,

930

* 1520 ifushed Sample FRE $1 H P-4$ at 1520 .

* 1550 Pushed sample FRT -1HP-5; 10 te $115 f T$ ot 1550 contact from sand to $C$ lay atoll \& T.

$* 1615$ $* 1650$
Push Sample ER Flit plo at $1615,15.0$ to $16.5 \mathrm{ft}$

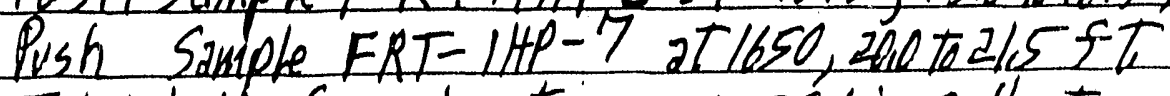
Total depth of exploration: $21,5 \mathrm{ft}^{\prime}$ collect 7 samples (603" split spend 10 2" split spoon). Auger to $20 f T$. Sean collected a replicate Append IX IX BNA at Sample 4, Sean and I check samples, tape labels, ne pack samples, lack, ate and 1800 depart for We II BId at 1860 . the mall truck, lack 18,30 samples in cage,det Shes at 183n,
$3 / 13 / 91$ Daily Activities Report

0645 Meetisean Asquith and C.B. Travis, at 1645 -proceed taste, o700 Heavy rain at site, meet Dan Wells at Well BUlg 0230. Janelle Jansen notified by Graves well Drilling that Graves prefers to not work in the rain. Janelle said it is Graves" call, Janelle said Tom Gavahnis Making shipping arrangements far

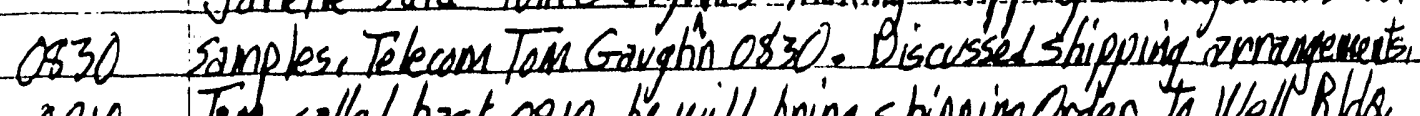
0910 Tom called back 0910, he will bring shipping Order to Well Bldg e 0920 Talked to Trace Bister are Tandfl Jansen and concluded that I should take samples offsite ad ship then
1200 1215

1245

1253

si

st.

5

1150 
5

fill bottles

on on

assuming

requiring

"so been myself rather then back then at shipping i Receiving for then to

930 sean prepare chain of

-ft + t 1550

255 custody

Pack samples mf bubble

$15.01016 .5 \mathrm{ft}$

ate $215 f T$ -elect

$=$ "split spoon).

ind I check

-k. gate and

bad truck, lock. upas

1030 Telecoms tom Ganglion re i final arrangements far s tipping samples to dab. He has a runner hand carrying shipping documents armand for signatures - will deliver to Sean at Well Bldg.

1150 . Finished pattering samples. Enclosed $140 \mathrm{~mL}$ VOA Trip Blank in the shipping cooler Wait for Ton vaughn to come by wren the shipping order.

1200 Lw neh.

1215 Tom Gaughum brought

reed is sites b) the shipping order 1245 Lw well Build rye

i 4730 . Janelle

craves prefers

"raves "call. 1253 : Any shipping Called to Bobby sims. arrangements for He said the would be here $\therefore$ ing a rramemets. in about 10 minuets

or to Well Bldg

1335 gu shipping

rel Leave cooley with nelarke under uples 1815 shipper samples Fed $=x$ 


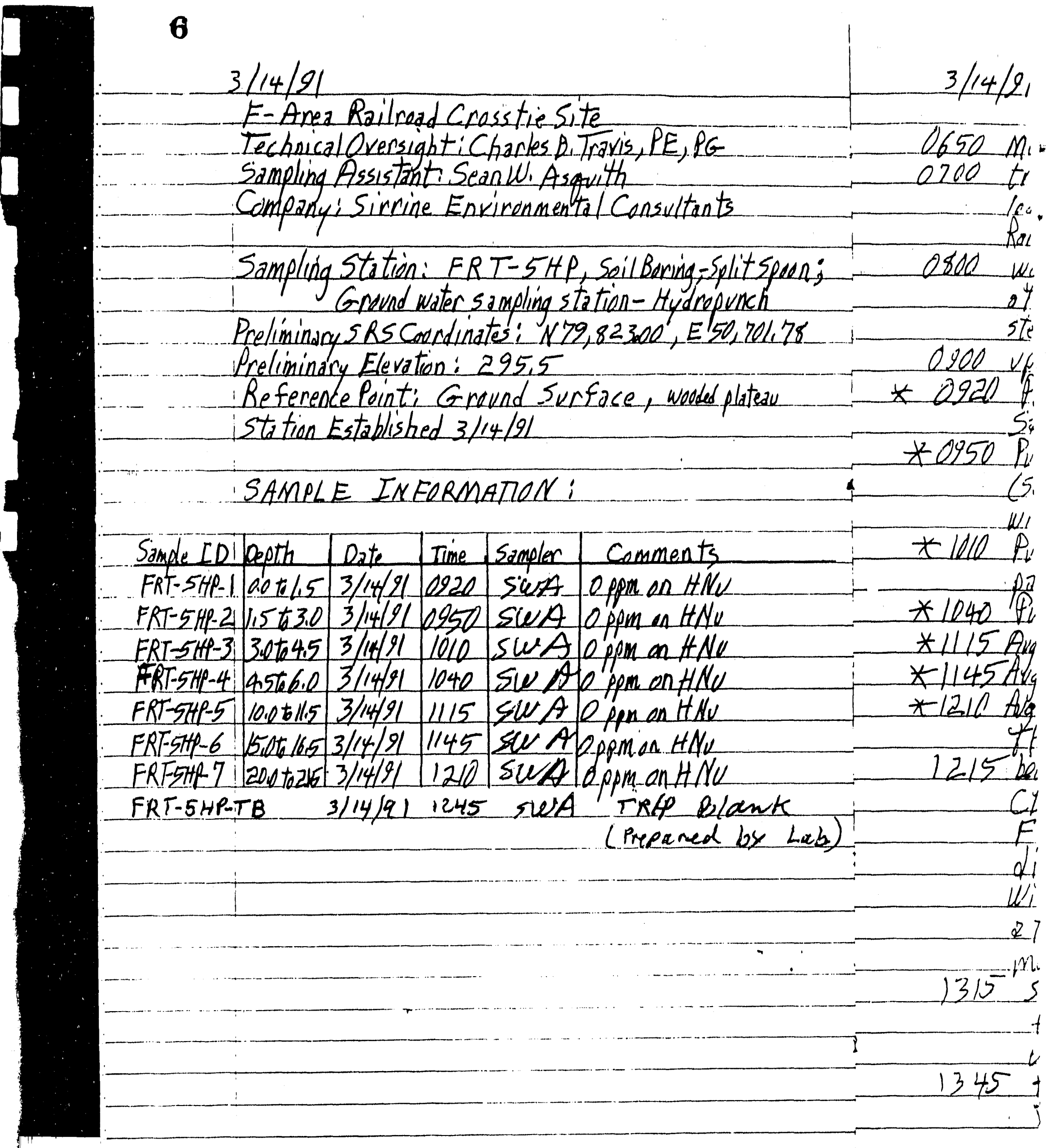


7

$3 / 14 / 21$ Darly Activities Repart

if $\quad 0650$ Meting SirrinelXfFice-CBT+SWA,Ledd equipment on

ints

-iplitspens;

arkh

$.0,701,78$

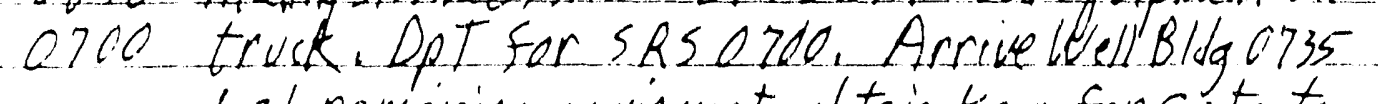
Irad remaining equipment, abtain. Key fer Go te to Railread Cresstive Aned. Fill centainerstwith D. Ir rinse.

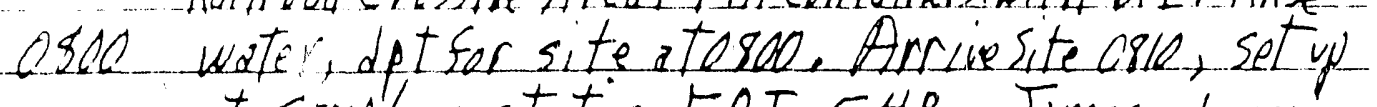
of simpling s tatien ERT-SHP. Temes and crew steam.clean a uqeers, eter at statien FRT-lHP, set C900 up rig at St ERT-5HP at De20, ceadut sample

: platezu * O2al Push first Sample, FRT-5HP-L at ig2a. Decon sampling east. Pack samples (swA), prepare to sample * 0950 Pish ample FAT-5HP-2. ITO950. Lange cobble (sommin bitithesulting in partial ample, toweren, with 3 "split spon, sample suficicient.

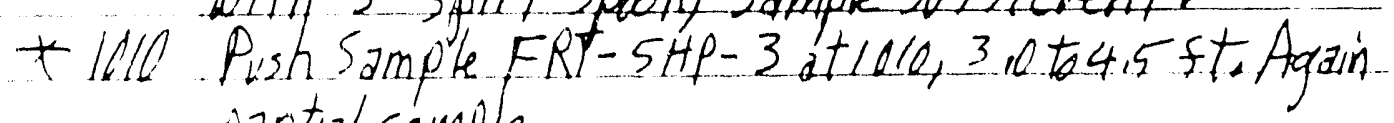

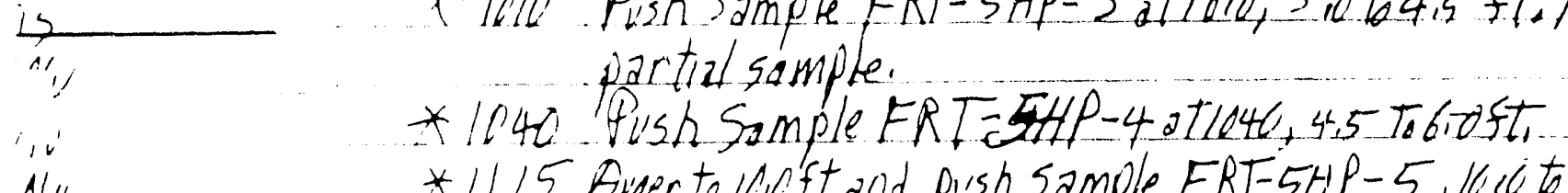

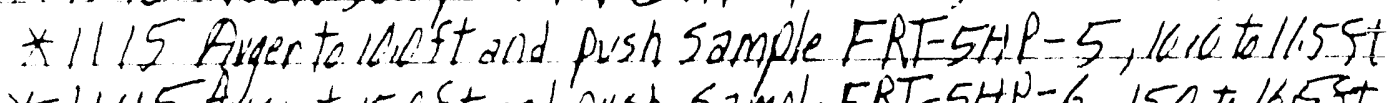
* 11.45. Avgertorisa ft and push Sample ERT-5HP-6, 150 to $1653 \mathrm{st}$

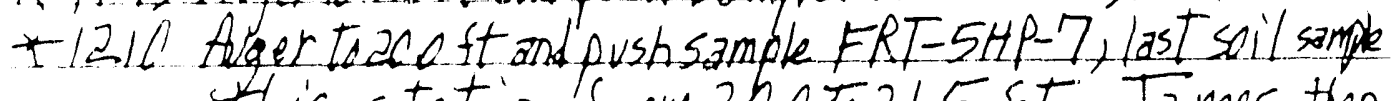

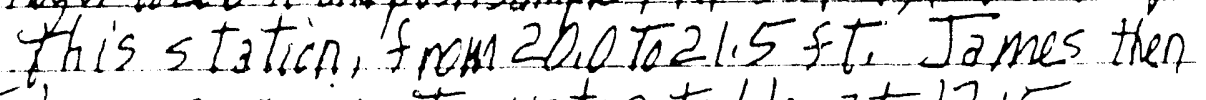
1215 began augering to water table at 1215 , CBT : iempts to check wiater level in wells. $F B P-3 A$ and $2 A$. Cld style well with small dianieter drep pipe; Watei level indicator Will net fit ith side, CBI dpt far well Bldg z 1245 for different waterlevelindicator -inere di I, water, ets

$1315^{-4}$ sam $g_{0+}$ toguther botfles for nater soupling Cleaned up a tuter soil saupling.

1345 th Decan Hodiagunch ard baller. 
8

$3 / 14 / 91,($ Continued)

CBT review well dato with Bobttunter. Ascertain That wells FBP-2A and $3 A$ may not be water table. wells based an screen settings se Return to

1350 site of 1350 with second water hevelindicitorJames angering at $70 \mathrm{ft}$ cuttings moist, but net saturated, second waterkerel indicator still Ter large to fit drop pipes on Wells $F B P-2 A+3 A$. CBT radio R. Hunter. SuggesT angering until saturated cuttings noted, Then clean inside of augers with decontaminated 3 "split spoon and checking for water inside The augers with The water he rel indicator. Noted color change in auger cuttings at $=8 z^{\prime}$. Cuttings progressively damper but bot saturated. Hunter concurred. Noted drilling change at 95 ft, cuttings dame, and no mare a voe rsi Trifled in 150013 "split speer a 1500 , split son en a bottom of 1515 auger at 1515 ; perhaps 3 "fill inside auger. Push 24 ", then Trip ont.

1520 Sean calibrate pt t meter 107 with 7 and 4 buffers 1525 Tripped split spoon out at 1525 , rods and sample dry,

We reading oh water level indicator + James contacts Graves via radio. Bruce advises James to pull out 40 ft of augers and dag it eff until tomorrow CBT 1535 advise Dan Wells via radical 1535. sw A repacks hydropunch, Reloddgear on J ruck reconcile Subcen track r expense record with driller. dpt site for Well. Blog at 1665 . Off load truck, packsamples, refill DI wot er containers, et k

1645 Sean fill -out chach=ot-

1710 custody Leave cooker ot samples locked in cage at well building 
9

r. Ascertain be water table: Turn to indicate rmoist but cater still op -2A+3A. , until matured tors with risking fir - ter level a er cuttings stiver buthot " change at si. Trifled in betting of alger. Push

veter Leers 1 sample dry. ne's contacts to pullout morrow. CBT . SW A repacks wheontractor "ell Blog at DI water

$-0+$

tossed with custody seal on cooler.

1.715 D PF IRS 1715

$3 / 15 / 91$ Dally Activities's Rejort(FRT-5HP) 6650 EBI mut sw A O'S0, load Truck. Take tue vehicles to facilitate sample handling, etc. Meet James of

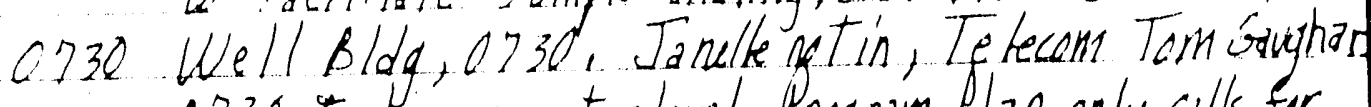
0730 to discuss waterlewel. Program flan only calls far drilling to $100 \mathrm{ft}$, but screens in FBP-2A+3A are 127 if below ground keel. Tom said ta drill as deep as necessary. 1988 water levels a but lo l st be bu grand level. Tom is preparing a shipping Order for samples. He requests that sean hamdarry The Shipping Cider ta Tracy Bitter far signature. sean contacts Tracy- she will nut be available a fatereaso

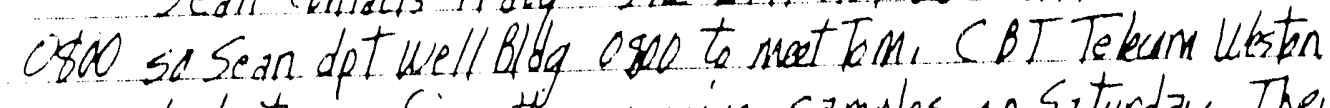
Lab to confirm they receive samples on Saturday. They cb, and will he expecting. a Fed Ex shipment tomerrick

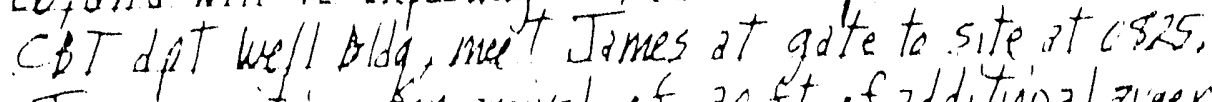
James wading ar arrival of act of additionalager.

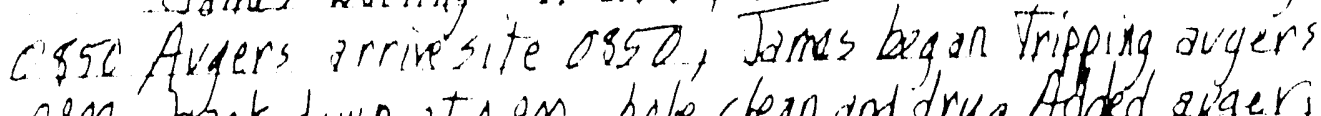
ope back dawn at os sob, hale che an and dry Added anger, drilled to las st, cuttings still mast, not saturated. 0220 Added augers-100-105etatogza. James reports soft drilling 122 - 1055t a Continue cleaning hale va min until saturated cuttings return ta surface. Ca attempt 5 check with waterkerlindicater but augers plugged it $164 \mathrm{fte}$ C BT asked James for sub to connect Hydripunch to rads. No EW Subarailable and hike in Aw Sub ter small for bailer to

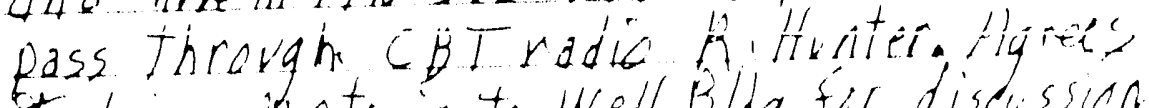
Fo being parts in to Well Blag fer discussion

ne 
10

$3 / 15 / 21$ (continued)

$3 / 15 / x$

SWA bark at site with shipping Order signatures. He will deon split ripen to Knock sail plug ort. aver. Meeting at gs 1030 Well Bldg ot 1030 -Ritunter, Bruce Kistansen and C8 Travis. 131024. The "EW" adaptor that came with the Hydrapunch does notmitch the description in the charts. Telecom Chris Bergen wa L100. for contact at QED, Telecoms Brad Peace IT QED $13402 r$

1100 at 1110 . The thread is EW Casing thread, not EW nod Thread. Bruce determined that an adaptor can be fabricated and shipped here by Tuesday. Telecoms Mary Flora re: overall. situation and the possibility of using, a temporary 2 "PVC piezometer. Mary $r d i$ will discuss with Bob Benson of DhiEC - he is out To lunch -will call back. Bruce get an estimate of $\mathbf{H} 100$ + tox and shipping with delivery saturday. R. Hunter authorized the purchase Fun the discussion about options. Discussed moving to on of the other boring and obtaining soil samples. Bruce points ont that, by the time we tan trip out of ERT-5HP, decor the augers, move and setup on another boring - we may obtain soil samples, but it will be too latte to ship them CBT Telecom Ben Shapiro 400 $2 n$ Th at Weston lab. If we sample today and ship Saturday he will get delivery Monday= This will be ok but is pushing it. It would not SB

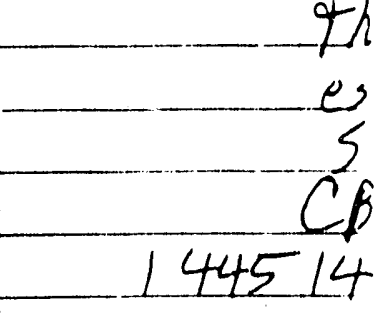
de acceptable to sample today and ship Monday. 1215 Radio discussion-CBT and Tom Vaughan at $1215^{5}$ Tom Concurs with maintaining the states Quo 1230 until Monday Telecoms Mary Flora-1230-She concurs with not mooring over, is we can proceed with Hedropunch Monday. Sw a neterustydreperch etc to well Bldg -locks in coge-dpts. with samples for shipgrigg, to 1245271245 . CB I return site 1255, James and Anthony 
11

$3 / 15 / 91$ (continued)

wis. He will Meeting at , and C es Travis.

are punch dee net -Gris Bergen ike it QED , not $E W$ adaptorean try. Tetecom , the pessililig inter. Mary EC-heislout 'n estimate. Saturday. Further moving to ming spit is the time in the augers. in - we nay ba tod latte on Shapiro day and 4 Monday- It would not -his Monday. ? an at $1215^{\circ}$ id os quo -3 0-She proceed with impeach, etc for shivering, grouting FRT-LHP To surface. Begin tripping, 1311 augers out of ERT-5HP at 1310 -after ing ing water at $102^{2}$ to in the hale. Complete tripping. 1340 augers out, at 1340 . Sealed the hole against rainwater infiltration with plastic and earth. 1400 and dpt site. Net Teresa Jardanat the date-gaveher the key so she could escort two other persons around the site. she will ne turn the key to Dan Wells. CB I return to well Bldg update files. Dpt site 14451445, SWA Shipped(and chard s amplesvia Fed Ex at 1800 . $3 / 18 / 21$ Doily Activities Report FRT-5HA 0645 CB T + SW A met at Siring of ice at 0645 , sean has prior sampling commitments, sa CBT will provide Technical oversight and sample Load sampling gear -drive b well

0830 Bldg, Laid remaining gear. Meet James a t site at of 32 tale caved at Q35 st, so no waterterel. James begins tripping in augers af 8940 while CBT decor new adapter Geneva machine al, etc.: Weather coll with light drizzle, 690 at 0900. Assembled of deconned Hydropunch. Completed tripping in

0930 Augers at 0930 . Steam cleaned ins de of drill nods one more time to begin trip ing. in. Tim

0945 Mckinsey visited site 0945 of assist with ha drop in Find rinse and assembly of Alydropunch-bogin. Tripping 1600 in at lock. Determined we heeded en addition $1040.5 \mathrm{ft}$ rod Anthony drive to Buckner's site - r th rout. sTeam cleaned assembled. Drove sampler (Hydropunch) $1445.55 t$ at 1045 . The bailer hits cacti calling 
12

$3 / 18 / 91$ (continued)

$3 / 12 / 21$

on the way out, Retrieving The bailer is very difflicyt and time consuming, and each

0815 Te econ. impact with a coupling unseats the check ball obtained sal that, by the time the bailer is at the surface, it is empty. After three attempts. asked ficzamete. Jan es to call an Graves nadia to have ole. in place Jansen or Robert Hunter visit The site and Groves that w. observe the opera Tin. Dan We Ils arr site 1100 , followed by Rick Frey. We again attempted to sample It as kit the Hydrgennch, but concluded that it will be necessary The wW flush joint casing . Tim, Lan, and Rick

1260 desartsite 1200 . Rick will han a clinch OD it is

1180 stainless steed bailer fabricated with no check exuemen; value and rounded repand bottom sa we can salvage This hoke, Don suggested Rick discuss No one: the financial aspect soy this with Rilturter cioler,e 1000 site 1060 . queened; from the

1235 or Janelle Jensen. Sean Asquith arrived site l1235. He depart site 1300 to obtain signatures for next outgoing $p l u g=0,4$ shipment of samples. Still no word on fabricating baiter apparent.

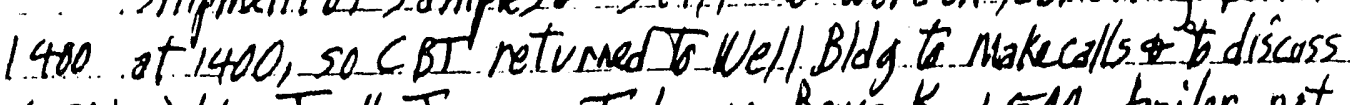
stan kyd tripping 1500 with Janelle Jansen. Telecoms Brie K. 1500, bailer not ready yet Groves will call back 2153 , Telecoms clean, 1545: Clank at Graves at 1545. Bailer should be ready \# sometime in the morning. CB T discussed with Janelle Janssen-L she said "legit off" far the day and don't come out in the miming until I confirm with Graves that the 1600 bailer is available. CB T return to site 1600, pack gear, reconcile hours with driller, return to well Bldg (630 af t site 1630 , a ft ir unkading gear. Note i. The drillers

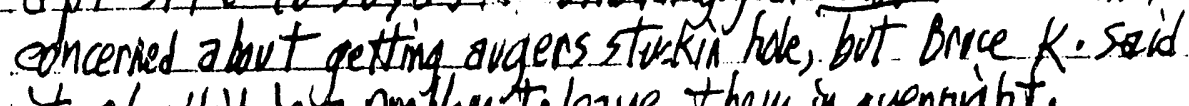
it shaves dea problem to leave them in oven night: 
1.3

3/19/91 FRT-SHP Dally Activities's Report

1 The baiter is

resuming and each Q815 Telecoms Janelle Jansen 0815. She has oats the check ball obtained permission To substitute 2" Temporary $r$ is at the surface, Piezameters in FRF 5 HP, 3 HP, and 4 HP tempts y asked in place of Hydropunch sampling: She said Queues waved gather z" prep and screen and that we should set them through the augers, I asked a bart filter pack - she indicated it is nat necessary. I toad sampling equipment purchase ice for shipping samples. it the site and

- Ils arr site 1100 , attempted to sample

tit will be nucksary
Tim, Lan, and Rick l - a finch OD 'd with no check

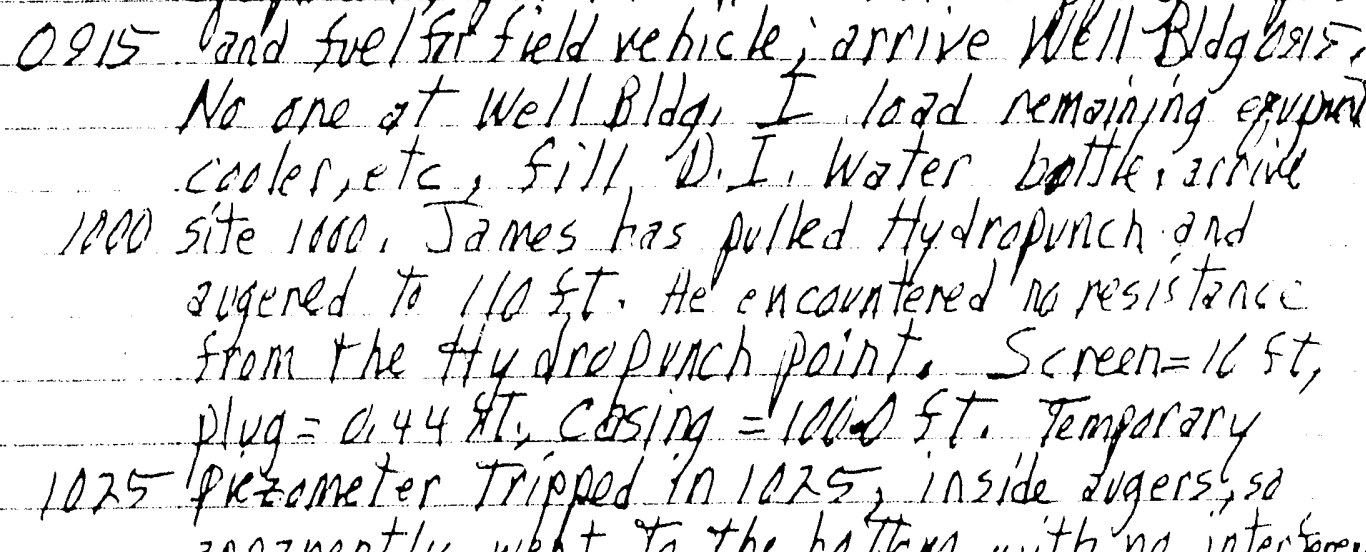

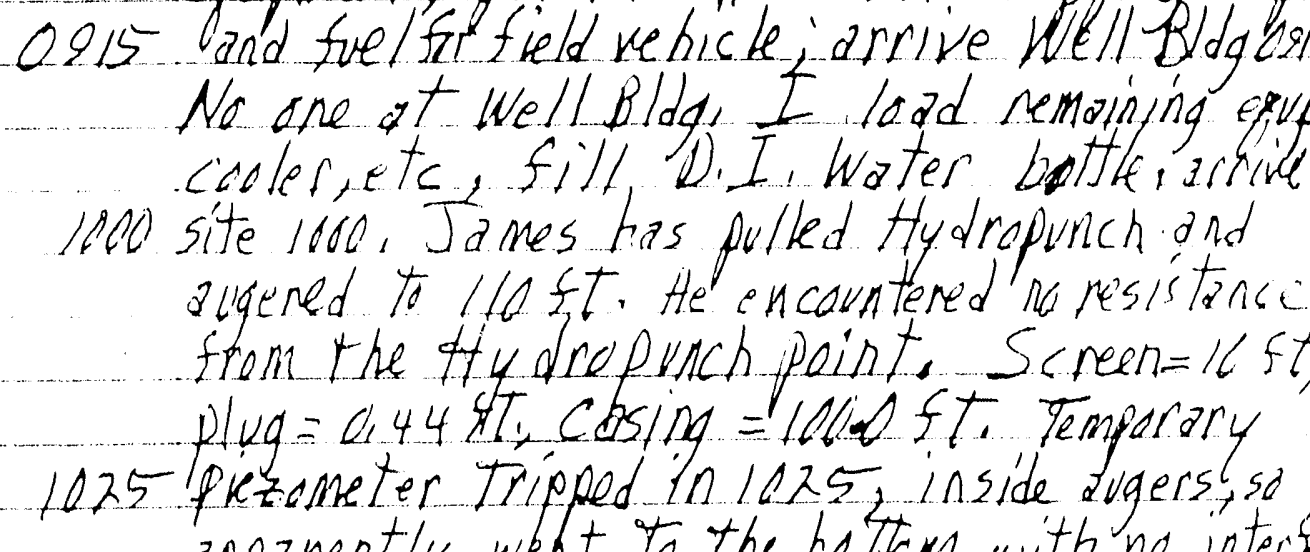

m so we can

- Ted Rick discuss lis with Rittunter with arrived sitelR3s. - Tor next outgoing ton Fabricating baiter a te nakecalls to discuss 5.1580, bailer not $36 \mathrm{k} \sim 1530$. Telecoms should be ready seed with Janelle Jansend don't come out

with Graves chat the ti site 1600 , pack $\therefore$ return to Well Bldg ar s Note: The dmilleris

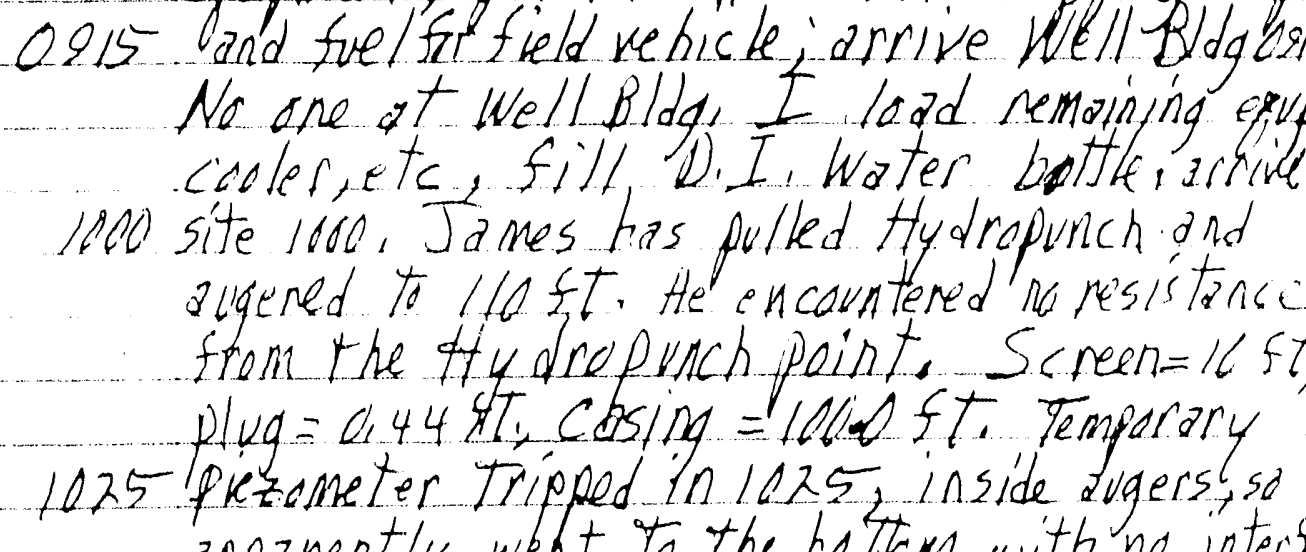

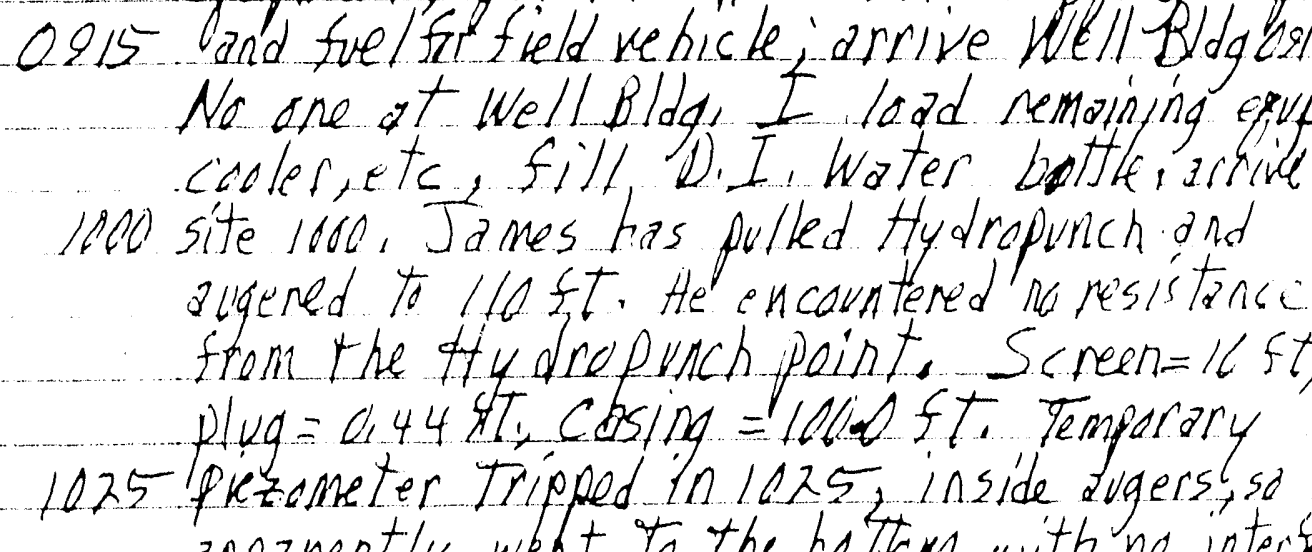

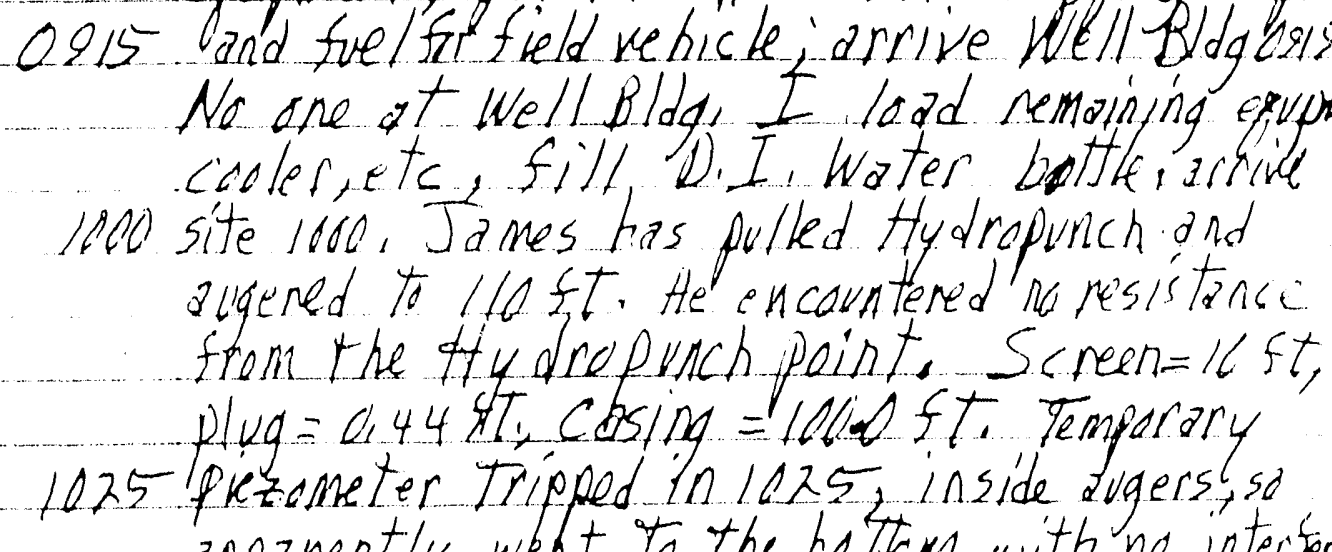

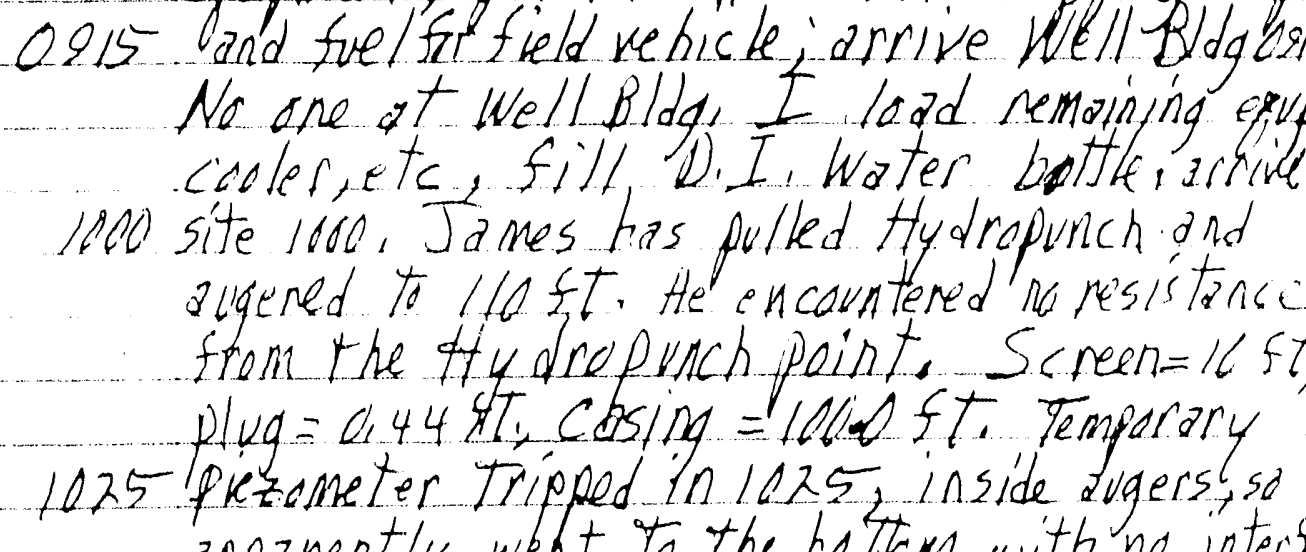
apparently wet to the better with no interierex. from Hydrapinch screen or paint. Began tripping aT a veers at 1030 a CBT disassemble, clean and ne pack Hydropukh. Augers out of 1055 hole at 1055 . Temporary the zoneter stick p $\sim 0.5 \mathrm{ft}$, so went to bottom as expected. Perm 1 60 down on rig st 1100 , Moved rig off hole, secured hole with plastic sheeting and sail. wised steam cleaner To deco rig, augers, and reds prior to moving onto next baring location James moved riquner state at II 30 while I set up sampling Station, James has boemuptready 4 in overnight.

1200 Tush first sample at 1200 . Sophie station getup attempted

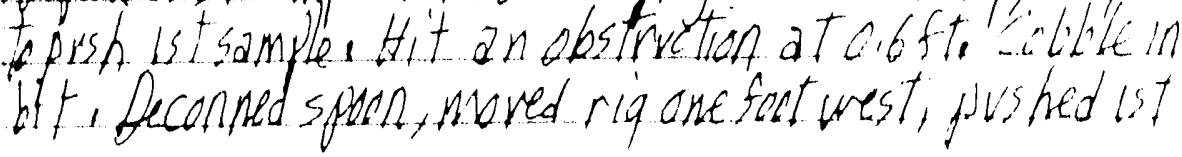


14

$3 / 19 / 4 /$ (continued)

$3 / 19 / 5$

* 1240 sample again. Pushed $1.25 \mathrm{ft}$. sufficient sample atinute

FlAre.

Augured to $1.5 \mathrm{ft}$ Pushed FR T-4HP-2 at 1315-V able

Technic,

to push doe to large cobbles - Auger 6 inches - Push again at

* 13201320 - to vas ft. Auger to 3.0 ft, push Sample

company

* 1400 ERT -4HP-3 at 1400 - 310 to 40 Et, unable to push

further. upper portion o sample damp, lower

portion dry. INv yelled 3 ppm in upper head

Sampling.

space, "O" ppm at bit. Augend to 4 s st, push

$T_{e, 1}$

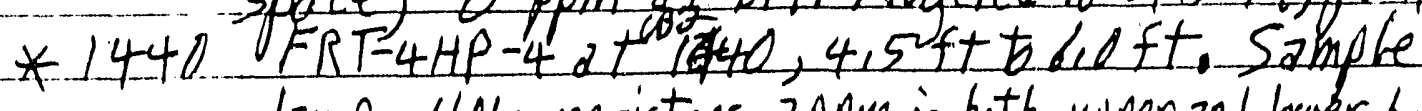

Prelimin

damp-HNo registers app in bath wp er and bower had

Prelims

space as split sion drive head and bit removed, Aliened

Refer

To lo. fT, Push Sample ERT -4AP-5-r: $15 \mathrm{ft}$-tchad.

Wo thing but auger cuttings in split spoon A Aver toll of $t$

Station

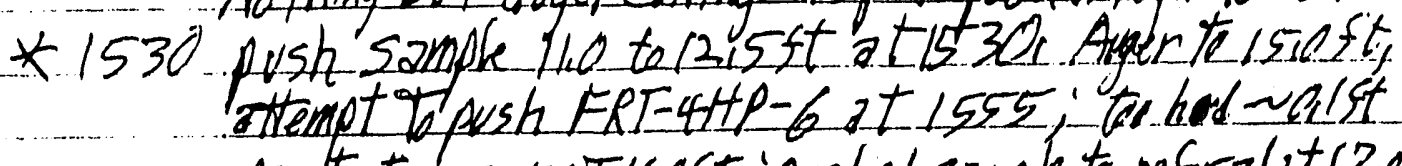

Sample

Sample ID Depth

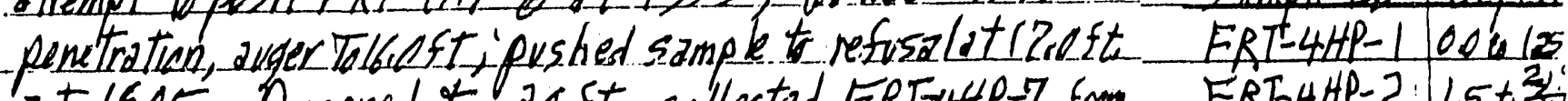

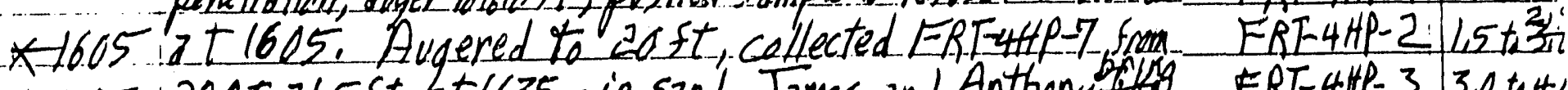

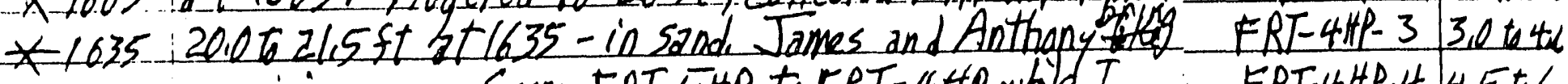
remaining augers from FR E 5 HP te ERT -Atp while I FRF-4HP-4 4,5 64

1650 : log 4 package samples. RT 1650 with augers. I note FRT -4HP-5

sample jar is broken- ERT-4HP-4, pH s sample. Cither jars fol, so should be sufficient sample. CB T completes pocking FRT -4HA-6 FRT-AHP-7: 200 t. 21. samplesjetc while James drills to 35 ste Reconcile FRT 4 HP TB:

i74.5 paperwork with driller, dpt site and lock gate aT 1745 R to to well Bldg, in lo gear, lock samples in 1815 cage, dpt well Bldg at 1815, 
15

- f ficient sample at into

tp-2 at 1315-vnable hes-Pushagalirat

s.lft, fush sample

, unable to push

iste damp, lower

hpm in upper head.

to 4 is fi,push

treeft. Sample

- ifpper and bourer huad

1 bitthemaved, Augered

$\therefore 52.15$ ft-toh hard

weon. Aver tolloft

- D. Anerterseft

is ter hod walst

wolet refusalat laeth lected FRF-uHP-7 frm

rames and Anthany ERT-4tP wite I

with augers. I note

pHsomplea Cther jars

1 completes packing

35 ft Reconile "lock gate at 1745
k samples in
$3 / 12 / 21$

E-Area Railrod Crassitie site

Technical Orensight: Charles B Travis

Companyi-Sirnine Environmenta / Consritaats

Sampling Stotian: FRT-4HP,

Soul bering-Split spoon samples

Temporary prezameter-baikd sampks

Preliminary SRS (aordinates; N 80, 181,21, E 50,442,81

Preliminary Elevation: 283,6

Reference Point i G reund Surface, drinage swale

Station Es tablished 3/19/91

Sample Information

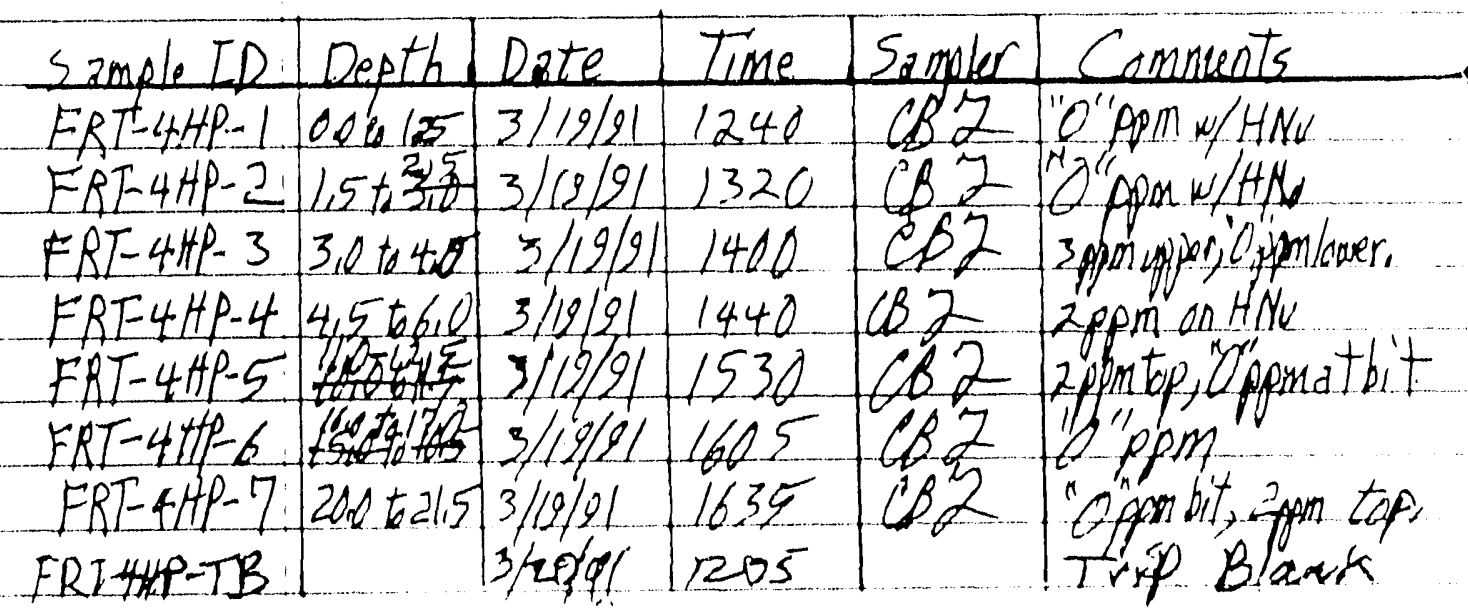


16

3/2o/21 Daily. Activities Repent

$3 / 20 / 91$

Q700 Load sampling equipment at Serine of fica oreo.

0730 CB T to Well BHgO730, load reluainihi gear and cooler

0800 of samples stroke cage. Mtg Janelle Jansen to discuss

progress. Janelle suggested collecting samples of grind

water from FRT -suP today because it might rain timorous

Q830 CBT fill $D$ I water bites, arrive site $0830 ;$ James angering at 65 st an FRT-4HP, CBT Packed samples from.

0215 yesterday in fresh ice. Teresa Jordan visits site 0215 0930, Noted a vg er cuttings increasingly damp below $25 \mathrm{ft}$, but not saturated. Saturated cuttings return vl10.5 ft -last anger $110-115 \mathrm{ftu}$ Cut hos wet but not a slurry. CBT believes water table alost. James not sure. Radio via Graves nadio-Bruce at Well Bldg. CBI reforests permission ti drill to $120 \mathrm{ft}$ and set $20 \mathrm{ft}$ of screen to be sure screen straddles water table. Bruce relays

1030 permission of 1030 , Drilled 115 - 120 ft by adding $5 \mathrm{ft}$ red on top of augers. steamcleanel nods and prc screen and casing (e") if station and

11.5 return 1115. Sean Asquith arrive site 1115, begins to wrap and prepare san oles far shipment. Begin Tripping 3

1135 split spleen inside avers at 1125 to make sure angers clear be fare inserting 2 "PVC screen. Sean dpt for 1130 Well Bldg 1130 To ob tin mo ne bubble wraf split spoon 1.135 to bottom of boring at 120 ft at 1135 , "began tripping out Begin Tripping in Temporary piezometer screen

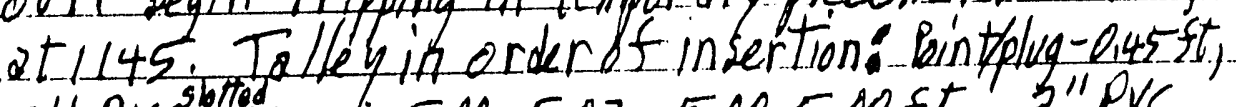

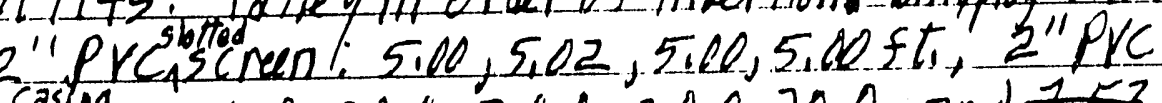

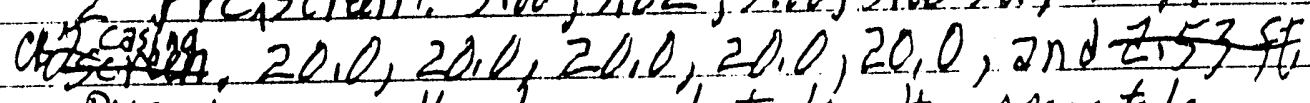
PV pine unvisally heavy, but does nt appear to be 1155. Schedule 80? I Son intusite 1155 a P casing stop at $112 \mathrm{ft}$-no need to use $2.53 \mathrm{fteiece}$.
1205 begin.

1245 out avi

1255 d f sta.

To ste di

Setup:

1340 station. boom $\mathrm{v}$

* 1400 at 140 Collect *1450 2I 144 Enable: shifted from F. * 1525i21 152 * 1555 . 00 to 11.5 Q +1555 push * $1645,2+164$ Killeen, 1630 at 1630 d * 1710 ERI = imateria. sand $T$ have b. of 27 25 st? with dpi

1745 site 17 . sampling 1800 ERT -4 1810i and at 1845 Samples 
17

art

$3 / 20 / 91$ (continued)

of fice a torca.

ining qear and coler

le Panssen to discuss

ig samples of qurvend.

se it might rain tamorions

'p sitec0830' James

CST pasked samples from.

indan risits site 0915

reasingly damp kelow

iaturated cuttings retarn

$5 \mathrm{ft}, \mathrm{C}_{t}$ itings wet

es water tablewllost.

ves nadio- Bruce at

mission to drill to

screen to be sure

ite. Bruce relays

if 115 - 1201 by

ugers. steamceand

'D") iff station and.

site 1115 , pegins to

TT. Begin Tripping 3

To make sune avgers

screen. Sean dpt for

' wrap split spoon

: 135, 'begar Tripping

plezometer screenate

Tion Bintolug-0,45st,

$3,5,0$ \&t, 2"PrC

20,0 , and टो 5 दf

csnt afpear to be

5. fle casing

$2,53 f$ tfiécer.

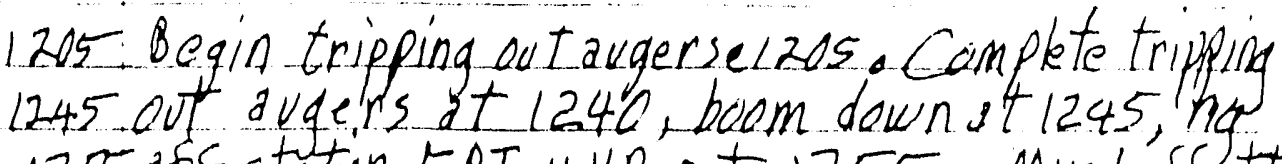

1255 of s station ERT-4HP at 1255 e Mared offos taton

To steam clean augers, cods,etc. $\left(B T_{1}+5 \mathrm{WH}\right.$

SeTup s amplingstation FRT-3HP. Rig on

1340

Station at 1340 plastic vider nig and boom up at 1355 . Push Sample FRT $3 H R$ I.

* 1400 at 1400 . Push sample FRII3HP-2 at 1425 . Collect rivsate sample, z@40ml VoAvials *1450 aT 1445, Push FRT-3HP-3 aI1450 Crable te cemplete sampling FRT-3HP in tirie to ship today so Sean opt site 1520 with samples Sram ERT-4HP for shipping. Push FRT-3HR4

* 1525: 2T 1525. Augerta lost posh FRT3HP 5 from * 1555 00t 11551 2t 1555 Augered To 15 feet Push ERT-3HP-6 from 15ip To 16.5 F * 1645 la 1645 a Jim Jordan, Natalie Park Terry Killeen, and a gentleman fram EPA visited site

16.30 ot 1630 , dpt 1645 Auger $7020.0 \mathrm{ft}$ push samigle * 1710 FRT-3HP-7 from 20.0 to 21.5 ft atiole, Material in the bit is fine grayish red purate zand This horizen has been Elemonstrated ta have background levels of lead on the order of $279 p M$ in M-Area, James augers to 25 St knacks off at 1730. Reconcile papormark with drilleri he helps load samples, etcon truek, dot 1745 site 1745 CBT stayson sithe to complete log, load sampling gear,etc, Nloasured us ter lewel in 1800. FRT-4HP 2T 973 st below ground level at 1800 1810 and at 103.0 Et in ENF SHP at $1810_{1}$ Deposit 1845 samples,etc at wellblogat 1830 . Dot SRSat 18450 
18

3/al/91 Daily Activitie's Repert

$3 / 20 / 91$

$E=\operatorname{Arez} R$

O730 Neeting CBT+ SWA at Sirrine of Fice at 0730 .

Eechicial?

Agree seian will us trick teday, preqane FRF-3HP soil

Simpling.

samples for shipment. CBT will use por, will aversee instillatin af temparany piezometer in FRF $3 H P$.

QBOB CBT arnine site Osbo. Antheny steancleaning augens of s.tation: Jomes argening atbo ft: having disficulty bringing cuttingst cobbles yf with sugens, CbT+ Janues. discuss waterterel-will drill FRE-3HPY) 105 st.

Samplin,

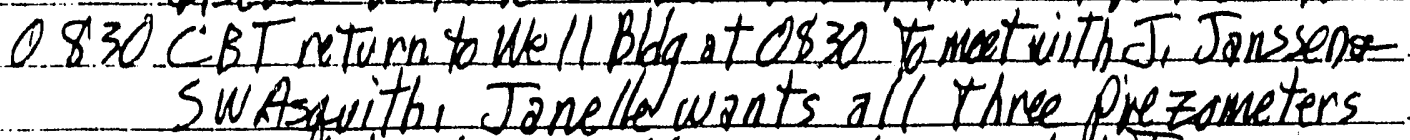
Prelimina Prelimin sampled Tod ay so they can be grouted Trmarnow: Referen I paint out That PRT-3HP isnde even installed yet, but will do what we con. CBT ossist SWA in

1000 assembling sampling gear-return site 1000 . Tanes

1010 Sean Aru ERT-5 HP setup - Sample TD Depth

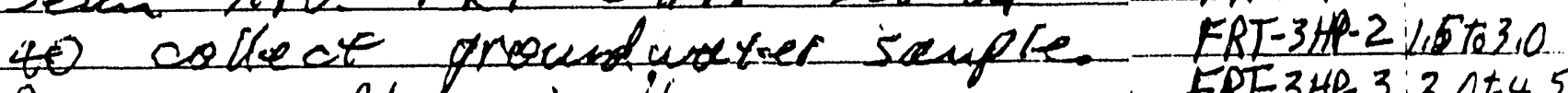
Decom teton backer ERF-3HP-3:3.064.5 1045 Calibrate got meter with FRT 3 HAP 4 4,5T660 7 and 4 buffers. FRT-3HP-5'10.6.61/.5 Get towher sayple contacines

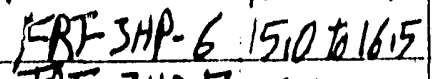
James dnills to $95 \mathrm{ft}$, cuttings damp stapdrilling TRT-3HRT2QQ62.15 1045 at 1045 to allow water evel to equilibrate ERT-3HP-R 1115 Nowater accumvlates; frill to ied ft at $1115-$ 1145 2 110 w to equilabrate-at 96 it at 1745 . James has $3 @ 5$ ST sections of screen, sO CBT directs Io drill To $105 \mathrm{ft}$ and set 15 fto f screen.

1230 Drill to $105 \mathrm{ft}$. Water aT $26 \mathrm{fT}$. T 1230 , Begin Tripging in Tem porary piezometer of 124 . table will allow collection of a cheaner, less disturbed sample with The bailer, becave 
$3 / 20 / 91$

19

EnArea Railmad Crosste Site

Office at 0730

; ReFRT-3HP seil

PQr, will aversee in FRF 3 HP

incleaning augens of

having difficulty

ivgers, $C B T+J a$ wes.

T. 3 HPY $105 \mathrm{ft}$.

- twith Ji Jonssene

three prezometers

1 1uted Trmarpow,

even instolled yet.

- ist SWA in

i.e 1000 . James
Tectmical Orepsiaht iCharles B. Travls, PE,PG.

Sampling Assistanti Sean W. Asquith

Company: Sinnine Enviranmental Consultantis, Ince

Sampling station i $E R T=3 H P$

soil boring, $3^{\prime \prime}$ split spaen samples

Temperarypiezometer-bailed samples.

Prelimáary SRS Cuardinates: N 79,872,19; E\$0,382.93

Preliminary elevation: 280.3

Reference Point: Ground Sunface,

Station Established $3 / 20 / 91$

Sample In Eumation

S Set-up

- reer sauple.

with

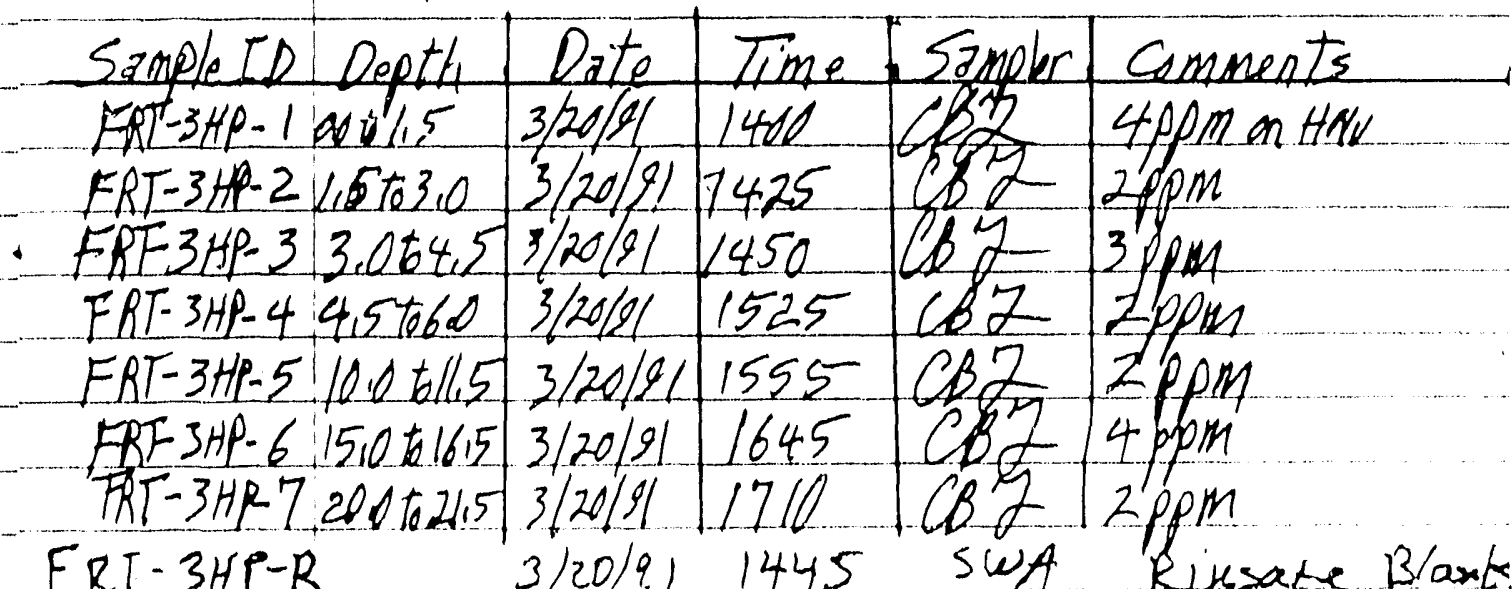

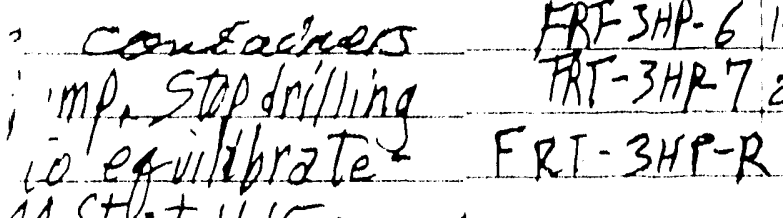

$11 \mathrm{ft}+1115$ -

$\therefore t 145$. James

$n$, so CBT directs

- 15 fte s screen.

fId 1230 , begin

- ter 25 1244.

$n$ below the water

citeaner,less

- bailer, because 
20

3/21/21 (continued)

$3 \mid 21 / 21$

Sediment will settle to the bottom of the screen. EAred,

1250 Pkzamater in place it 1250, Tames began

1315 Dripping out angers at $1315 \mathrm{hr}$.

sampling:

company:

SWA and $C B T$ bail ERT-SHP beginning at

1.145. Necessary to allow well to recover

about 5 minutes between bails with teflon

bailer To minimize sediment. Filtered

metals sample - l liter -through $0,45 \mu$

Sampling:

Nalgene filter apparatus. Complete filtration Depth to water 103. at 1325. Complete filling / $/ 2$ gallon Rad Di te $3 / 2$

1330 Sample at 1330 . Complete packing samples Time

1345 and move of sampling's station at 13451 . Vol Purged 3 Ba

Note: Water level at 103,0 st below ground surface oft 5.3

prior to sampling:

$14+10$

second 65.2

$F$

ERT -3HP-2t 14 lh. Water level $28.5 \mathrm{~s}+\mathrm{TCC}$ Alkalinity 4.4 with $3,7 \mathrm{fT}$ stick up ais 94,8 it below $G 5$.

(ma/bas $\mathrm{CaCO}_{2}$

Sean setup on sampling station FRF-4HP at 40 , I Alkalinity, begins decontamination procedure. Wo ter level st

1435 9.7.36' below GS 2T 1435 ; Begin Tripping in

1440 first bailer at 1440 , Complete bailing and

filtering sample a 1640 . Setupon Station

FRT-3HPat 1645 econ and bail. comped

bailing and filtering sample at 1840 r

Note collected duplicate sample at this

station except metals sample not fitter d.

$\angle$ abe he d duplicate sample: ERT-2HP

1850 sWAt + CBT dpt site 1850 , far Well Bd or 
21

3 talal Water Samples E-Area Railnoad Cresstic Site Sampling: Siw Asquith, CB. Travis

e bottam of the screen
50, Tames begen
$15 \mathrm{hr}$.
T $5 \mathrm{HP}$ beginning at
well To recorer

bails with teflan
Company: Sirnine Enviranmenta) Consultents

Sampling Statiens: FRT-5HP; 1145 to 1330 ; FRT-4HP: FRT-3HP:

iment. Filtered

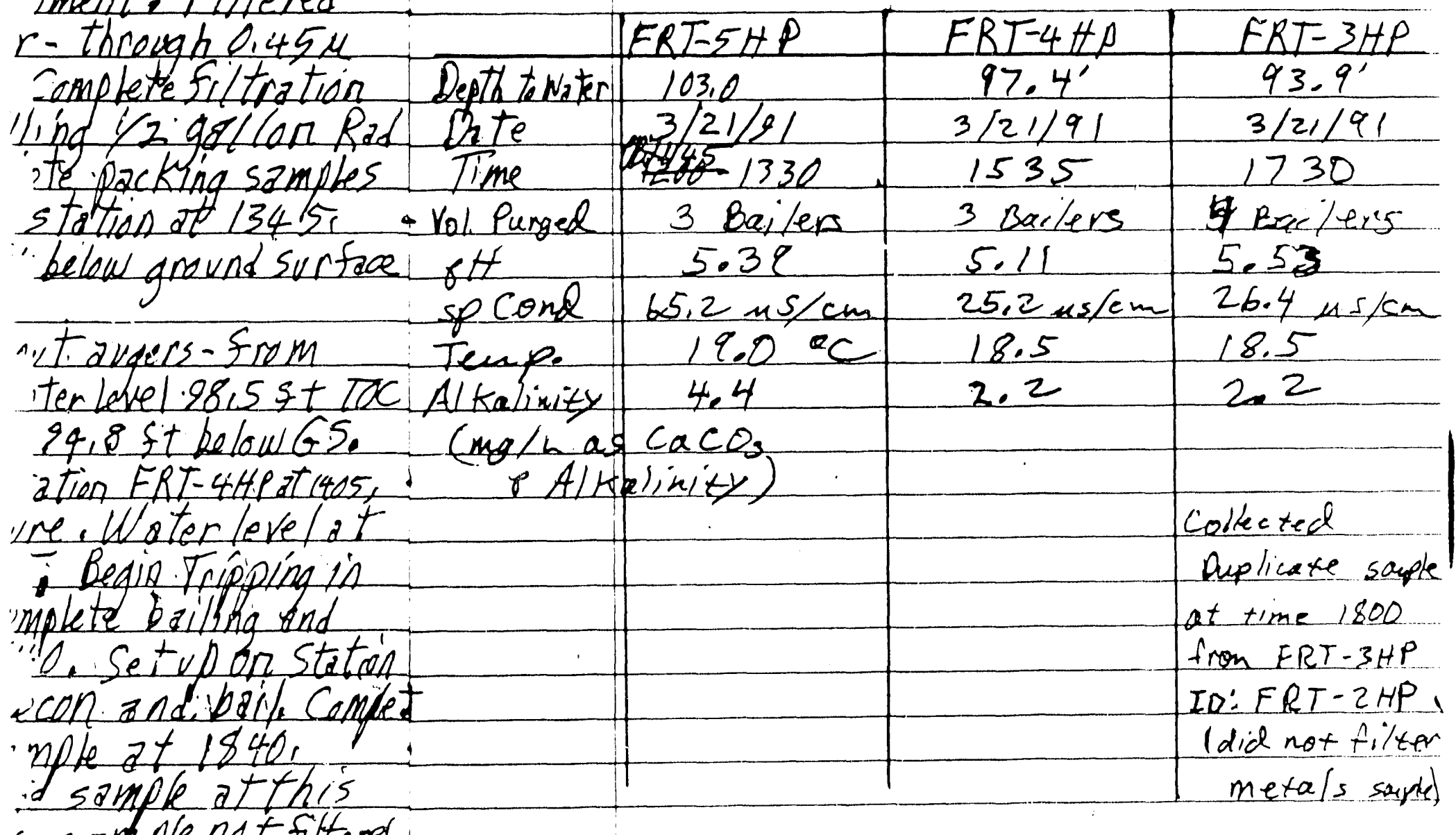

sample no t filtered.

- Mnle: ERT-2HP

1850 , far well Blágr 
22

3/22/91 Daily Activities's Repent

O 736 Telecoms Dan Wells Te C.B. Travis confirming technical oversight for grouting $E R T=3 H P$, $4 H P$ and
$5 H P$.

$3 / 2 z / 91$
Punch
s. ph

CBTon site. James has named PVC from

1800 Rod $F$

083 FRT-3AtP and is tripping in theme, Begin mixing

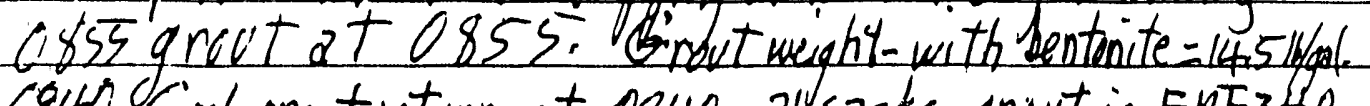

C940 Gond grout return af 0940, all sacks grout in FRE 3 HP Conitlete tripping out $105 \mathrm{ft}$ theme at $095 \mathrm{C}$ Flush grout from pump and theme and move onto

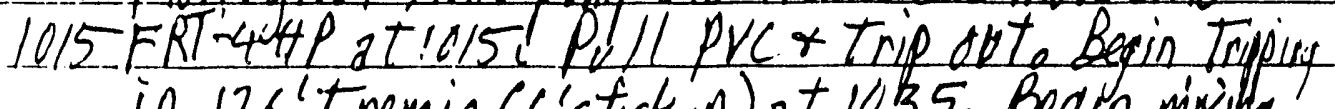
in $126^{\prime}$ Tremie ( 6 'stickup) at 1035 . Begin mixing 1130 Grout at 1045: 14,310 gel. Geod grout return at 1130. at 24 Sacksofgrout, Began tripping out Theme, From

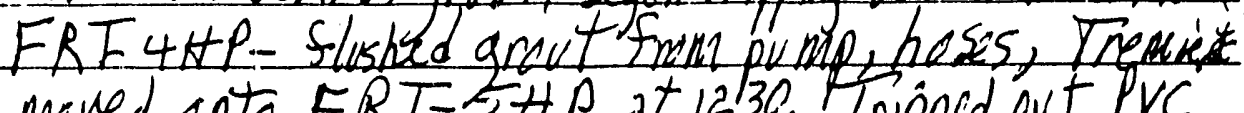
1230 moved onto FRT-2HP at 1230. Tripped out PVC at 1245 , began mixing grout at 155 - pump 22 sacks grouT o $14.3 \mathrm{lg} / \mathrm{ga} /$ with rectum 1330 at 1330 . Top out 105 to Tremble, flush Tremie, pumps, ho des,etc. C.B.T reconcile paperwork with driller. dpt far well Bldg with splits spent. logs, etc. Driller remains to load equipment and Dh ice site, FRT-3HP, 4 HP and 5 HP grout hos dropped $r$. $f t$ in each boringwill require topping of $f$,

CBT complete l soil Boring Installation Reports, updo te files. arrange shipping to Weston with Sean Asquith. Terry killeen relayed instructions 7 hrowah Janelle Jansen to ship duplicate sorites to lab (collected from FRT-3HAP and (aneled FRT-ZAP). WSRC will relay analytical instructions to Weston 1645 CB I deteRs 1645 with sample coolers. 
23

pert

$3122 / 91$ (continued)

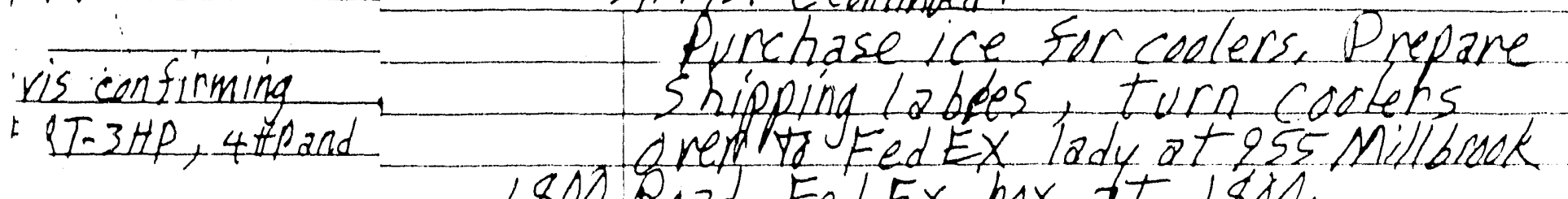

red Prc from

Begin mixing

1800 Road Fed Ex box at 1880.

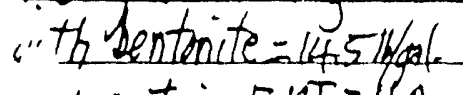

grout in FRF 3 HP.

Themien at $025 \mathrm{dr}$

idimave ionto

o duto begin Trping

$\therefore$ Begin mixilly

'evet neturn at 9130 .

isut Trenié, Fiom

mproses, Tremis

Iripped ou I IVC

1255-pump

lad with rotum

: es flusi Tremie,

resncile prenserch

with splis speons,

loadequpiment and

"P ahd 5 HP

in each boring-

Instelloticin

rnange shipping to

Jonssen relayed

- Collected from

LAP) WSRC

Tins to Westoro

rimple coolers. 


\begin{tabular}{llr}
\hline WESTINGHOUSE SAVANNAH RIVER COMPANY & Manual: & DPSOP 254 \\
Chapter: & $\begin{array}{l}\text { Page: } \\
\text { (Draft } 1) \\
\text { Of }\end{array}$ \\
SOIL BORING PROCEDURES & $\begin{array}{l}\text { Effectlve Date: } \\
\text { Organlzatlon: }\end{array}$ \\
SRS SOIL BORING INSTALLATION REPORT & $\begin{array}{l}\text { Environmental } \\
\text { Protectlon Sectlon }\end{array}$ \\
\hline
\end{tabular}

SRS SOIL BORING INSTALLATION REPORT

GENERALINFORMATION

Boring number ERT- IHP

Site E-Area Railsuad Cocsetie Pile

Boring purpose Sril Buring: fr

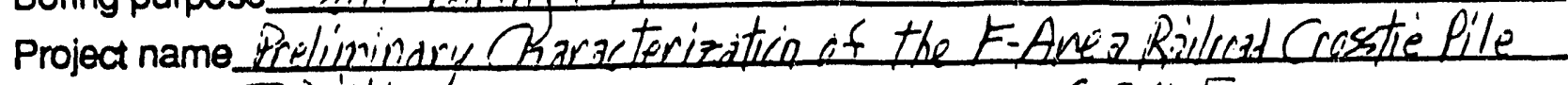
Custodian TBittled Custodial Dept C S KLE

SRS Coordinates: North_79,777.44

East $5(1,46,4,4$

Or: Latitude Longitude

Location description In a ditichldrawage suiale ldint iread

Drilling contractor Groves Driller Janies Snith, \#961

Oversight contractor Sirnine Envinenmenty/ OversightChorlesR.Travis/Sean W. Aseith Boring start date $3 /|2| 9 \mid$ Boring completion date $31 / 2,91$

\section{DRILLING INFORMATION}

Note: Depths are measured from ground surface to the nearest $0.1 \mathrm{ft}$.

Type of drill rig used Swik Drill

Depth to static water level N/it

Total depth of boring $2 / .5 \mathrm{ft}$

Ground surface elevation (mean sea level) $28,8,2$

Hole

Drilling Method

Auger

Hollowstem auger

1. $\frac{2 \frac{11 / 4}{1 / 4}}{1 / 4}$
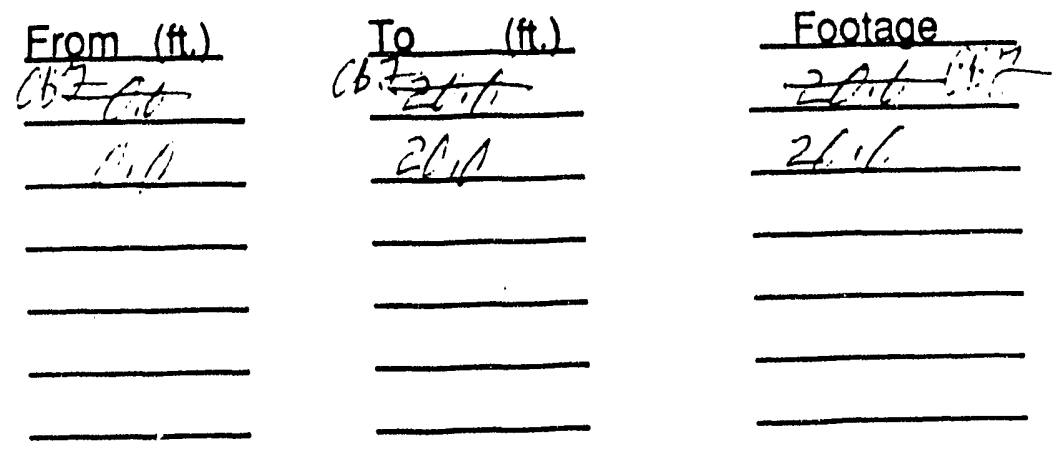

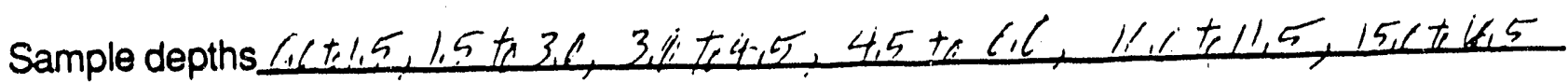
$266+21.5$ 
Split spoon:

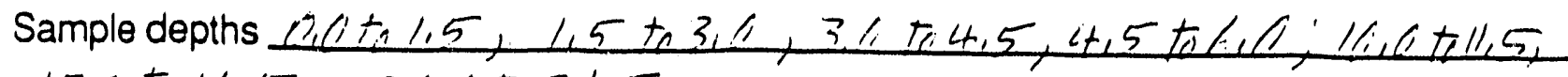
$15,16,5,241,21,5$

Other (specify):

Sample depths

Disposition of samples_ zhipkt "T. llisstall $\Leftrightarrow h$.

Tests condicted on samples st, TCLP.AnnIT

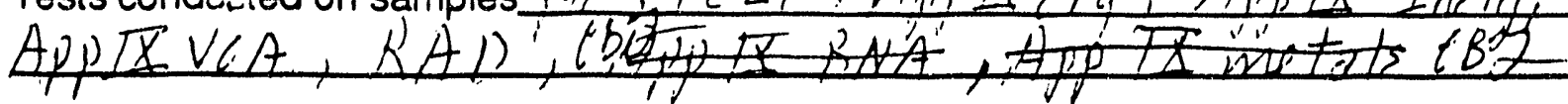

BORING ABANDONMENT

Material(s) used to abandon boring $350 \mathrm{~s}, \mathrm{~s}, \mathrm{riv} t$

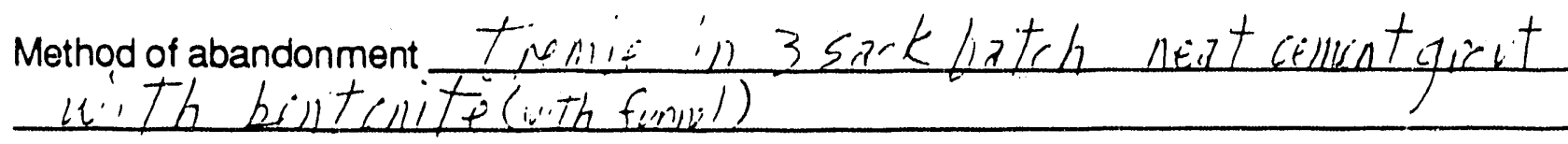

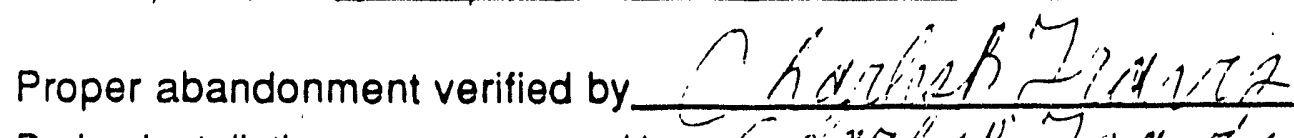

Boring installation report prepared by

Company sirrilli

Date

191

\section{COMMENTS}




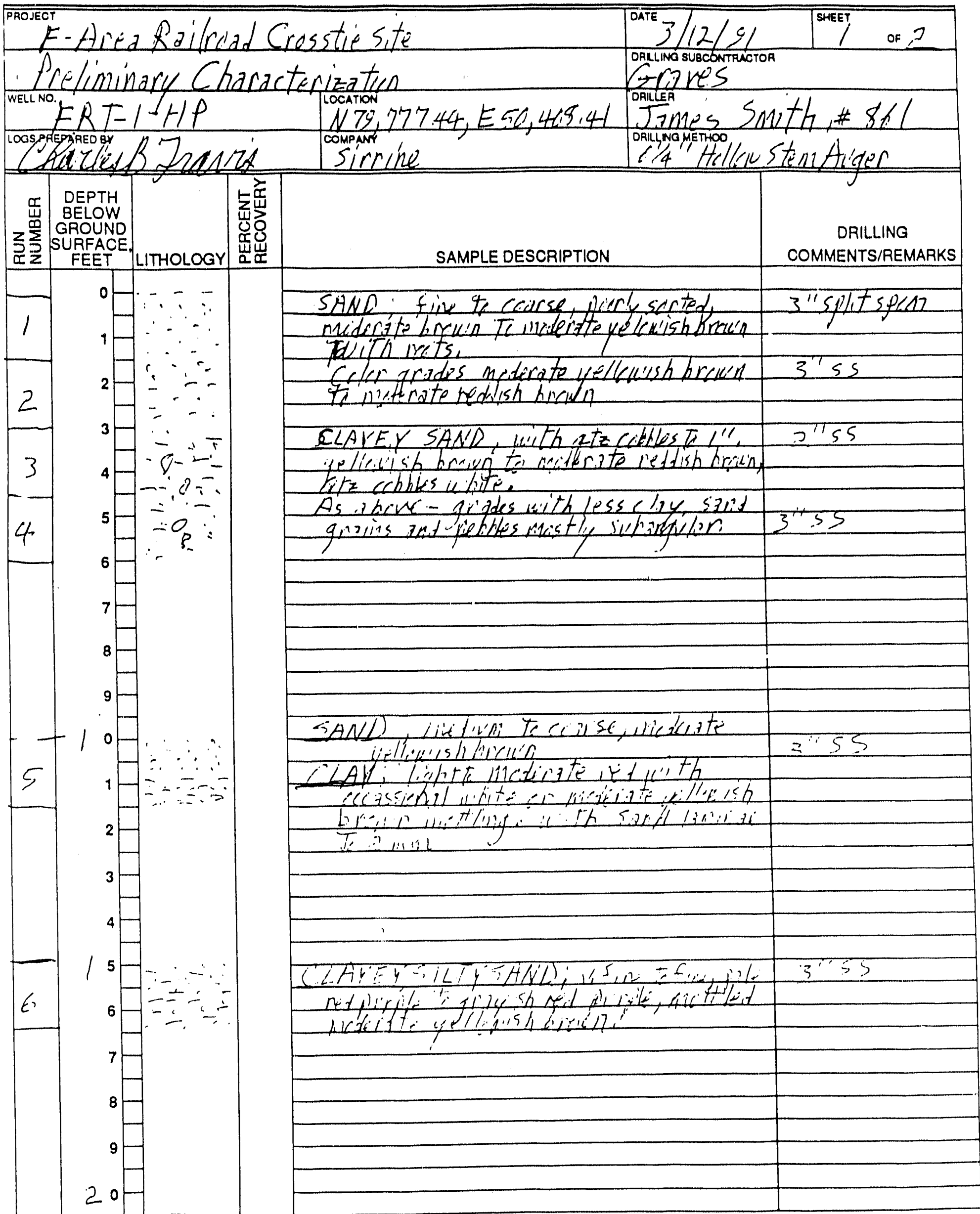


Foolect Ened Rairead Crosstie Site Dare $3 / 12 / 91$ [ster 2 of 2 OALLINO SUBCONTRACTOA DRILLER

\begin{tabular}{|c|c|c|}
\hline WELLL NO. & LOCATION & 0 \\
\hline LOGS PREPARED BY & COMPANY & 0 \\
\hline
\end{tabular}

ORILL NG METHOO
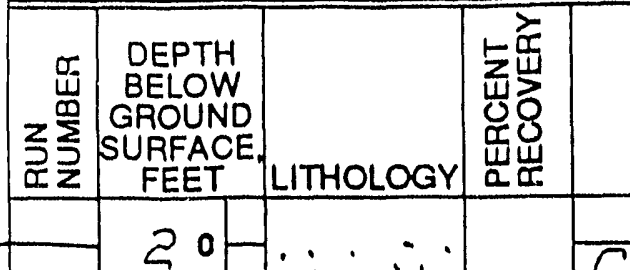

DRILLING

SAMPLE DESCRIPTION COMMENTS/REMARKS

$20 . \div \therefore \therefore$

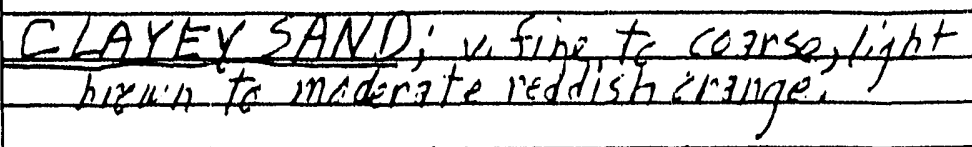

$1+\div \equiv \equiv$

2

Iotal Aepth of Explaiationials st.

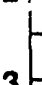

completed 1700, $3 / 12 / 191$ 


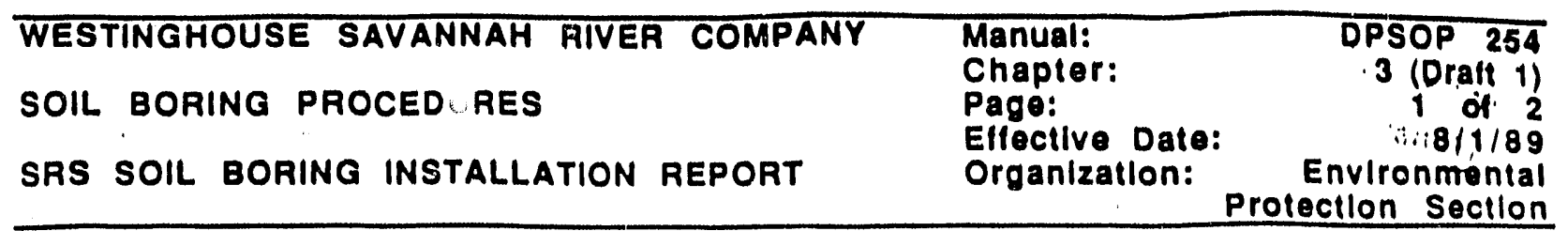

\section{SRS SOIL BORING INSTALLATION REPORT}

\section{GENERAL INFORMATION}

Boring number ERT-5HP Site F-Area Railrazd Cresstie pile Boring purpose scil luwing with aider Toble salunle

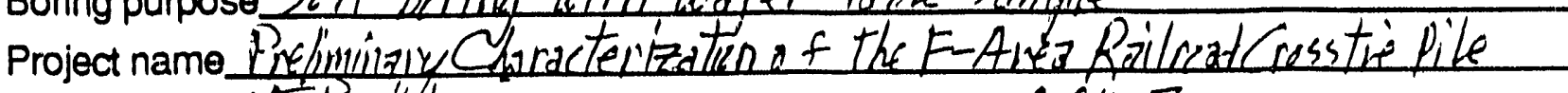
Custodian T,P,tler SRS Coordinates: North 78,923,4 Custodial Dept CSki E

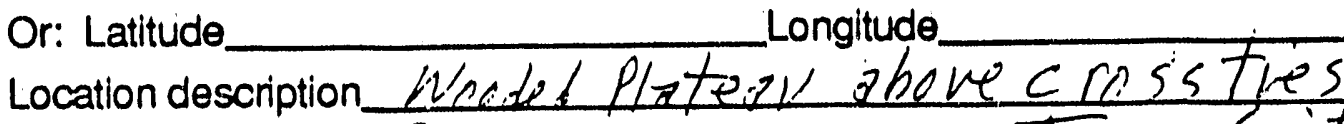
Longitude

East $5 /, 74178$ Drilling contractor Oversight contractor SinNike Boring start date $31 / 41 \& 1$ Boring completion date Driller Jamesmith, \# 861 Oversight C.IBITavis

\section{DRILLING INFORMATION}

Note: Depths are measured from ground surface to the nearest $0.1 \mathrm{ft}$.

Type of drill rig used.

Depth to static water level

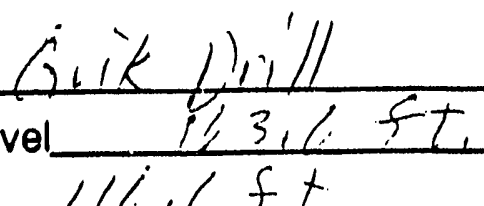

Total depth of boring Ground surface elevation (mean sea level) $2.5,5 \mathrm{ft}$ Hole

Drilling Methed Diameter Auger

Hollowstem auger

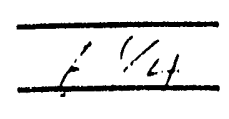

Mud rotary
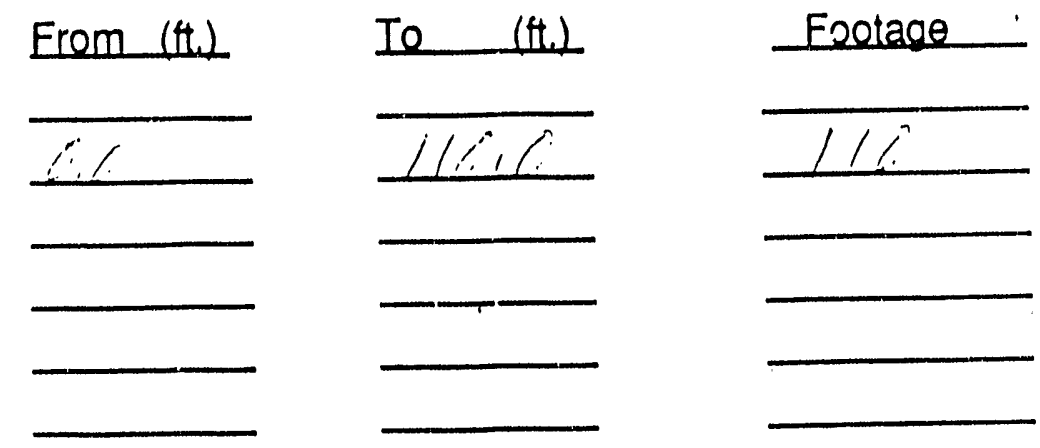

\section{Sample depths}




\begin{tabular}{llr}
\hline WESTINGHOUSE SAVANNAH RIVER COMPANY & Manual: & DPSOP 254 \\
Chapter: & P (Draft 1$)$ \\
SOIL BORING PROCEDURES & $\begin{array}{l}\text { Page: } \\
\text { Elfectlve Date: }\end{array}$ & 2 of 2 \\
SRS SOIL BORING INSTALLATION REPORT & $\begin{array}{l}\text { Organizatlon: } \\
\text { Environmental } \\
\text { Protectlon Sectlon }\end{array}$ \\
\hline
\end{tabular}

Split spoon:

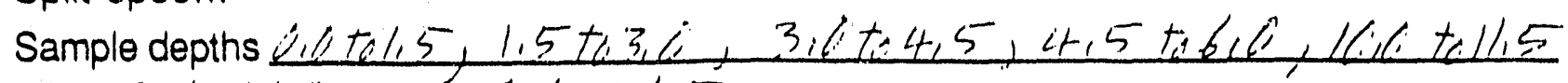
$15,1616,261,21.5$

Other (spocity): Witer samule ot water to ble

sample depths it 1 , ft

Disposition of samples Shiffed the $5 \tan <7 / 2$

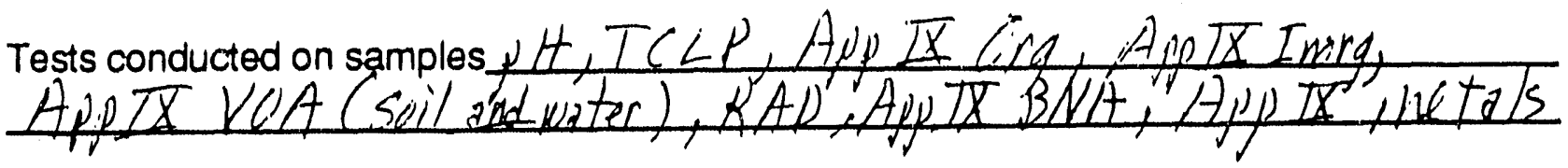
BORING ABANDONMENT

Material(s) used to abandon boring Neat cekunt gravt with Bentonite

Method of abandonment Prmp greit thrugh Tremie

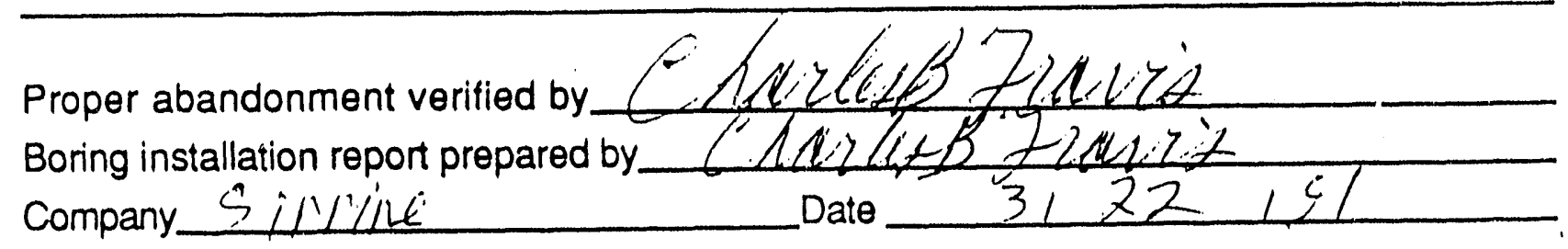

\section{COMMENTS}


Fo-Ared Roiluciad Cresstie site

\section{Prelíminary Charicterization \\ FRT-5HP}

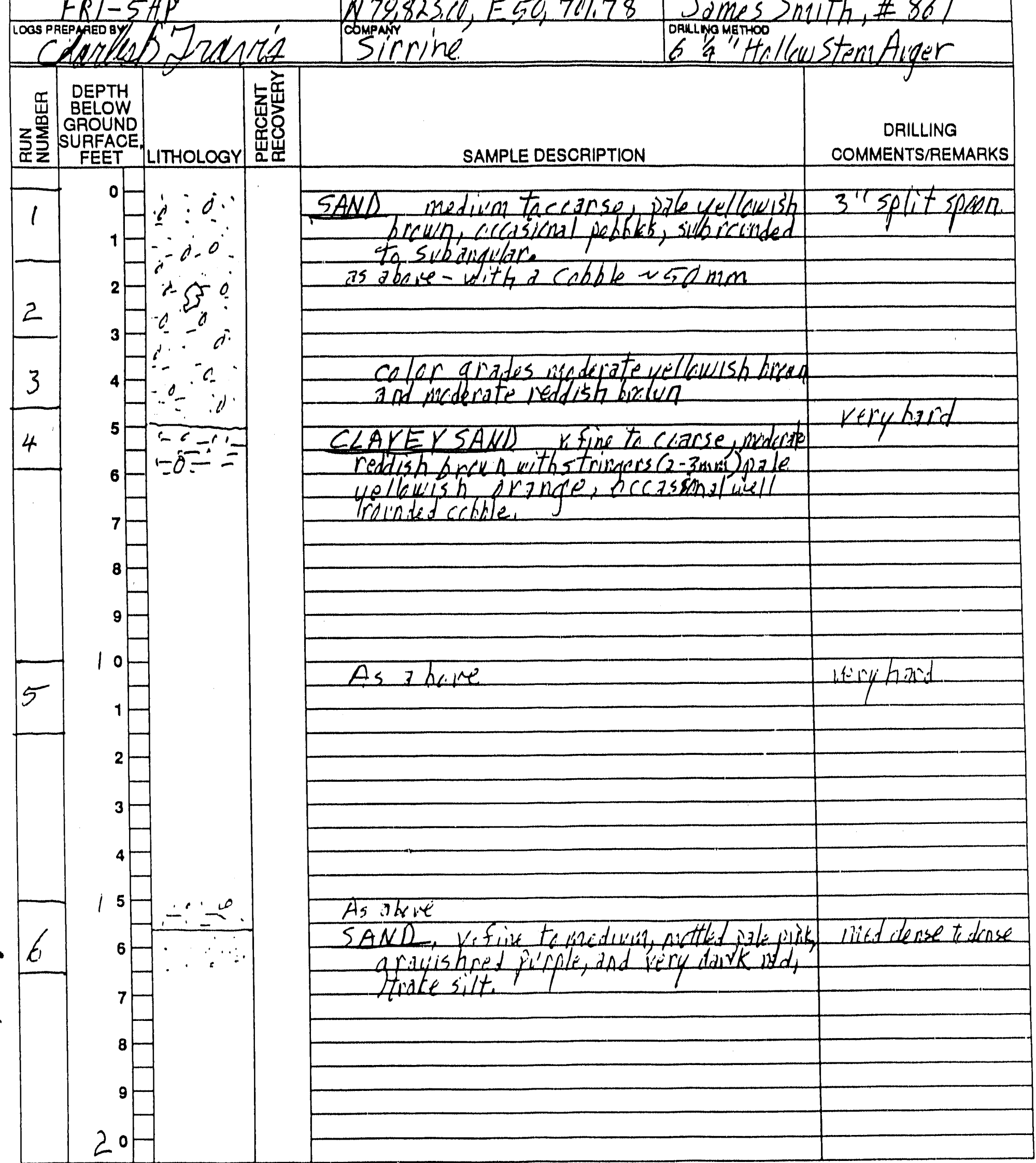
orling mentioo
$3 / 14 / 41 / 6$ ore $/ 2$ DFLLING SUECOWTPGOTOR Graves DARLER 
FIELD GEOLOGIC LOG

poosofo-Area Railinad Crasstie site

WELL NO. FRT-5HP

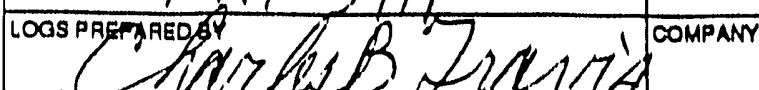

LOCATION

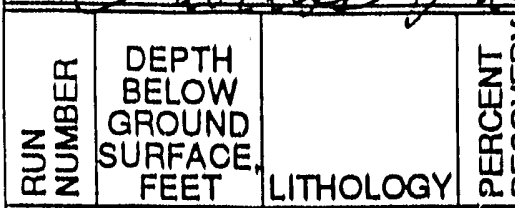

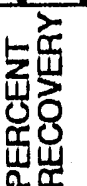

$3 / 14 / 41$

DRLLING SUBCONTRAOTOA

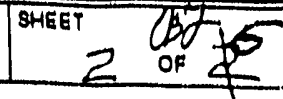

DRILLER

DAILLNO METHOO

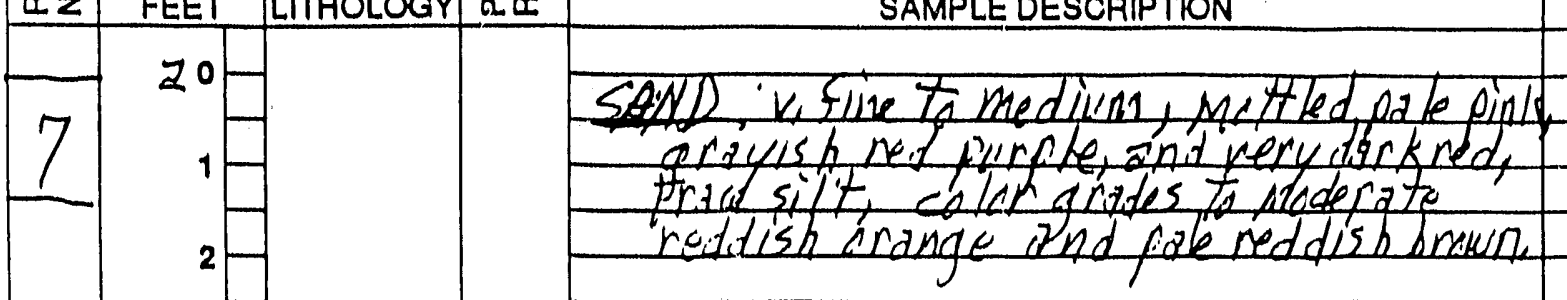


FIELD GEOLOGIC LOG

PAOEOT-Area RailradCress tie site

WELL NO.

ERT-5HP

LOOS PREPARED BY D OI

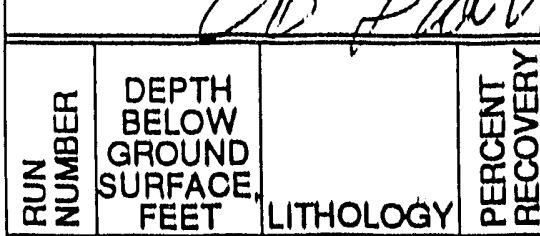

COMPANY

$3 / 14 / 91 \quad 3$ of 6

OALLING SURCONTRTOTOA

DRILLEA

DAILLNO METHOO

\begin{tabular}{|c|c|}
\hline SAMPLE DESCRIPTION & $\begin{array}{c}\text { DRILLING } \\
\text { COMMENTS/REMARKS } \\
\end{array}$ \\
\hline $\cos ^{2}$ & lexged \\
\hline SAND vifine to coarse with $15-15 \%$ & Frent Avear \\
\hline 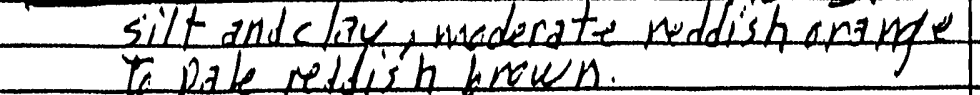 & cuttings \\
\hline Th pate redish known. & $T$ \\
\hline & \\
\hline & \\
\hline & \\
\hline As a have & \\
\hline & \\
\hline & \\
\hline & \\
\hline & \\
\hline & \\
\hline & \\
\hline As jhane & \\
\hline & \\
\hline & \\
\hline & \\
\hline & \\
\hline & \\
\hline & \\
\hline ifs i have & \\
\hline & \\
\hline & \\
\hline & \\
\hline & \\
\hline & \\
\hline Eter arates uth lesend 162 & \\
\hline 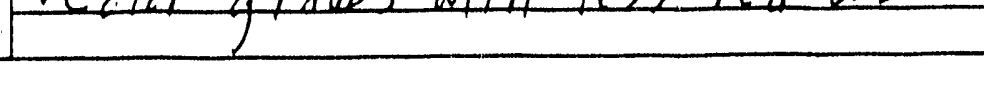 & \\
\hline
\end{tabular}




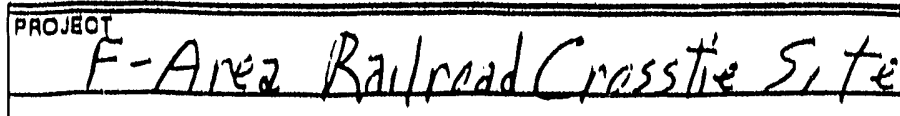

WELL NO.

ERT-5HP

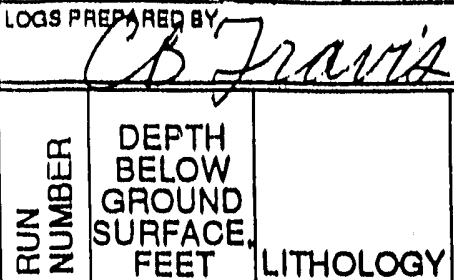

SAMPLE DESORIPTION

DRILLING $60 \mathrm{~S}$ COMPANY

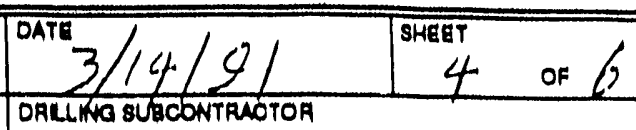
DRLLLER

DRILLNG METTHOO

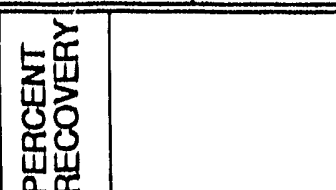
COMMENTS/REMARKS

SAND rifine to coarse with lato 157 silt ind $\mathrm{C} / 34$, inderats bergat redish crange to pale weddish ceitting As Abave

As a tiake

70 


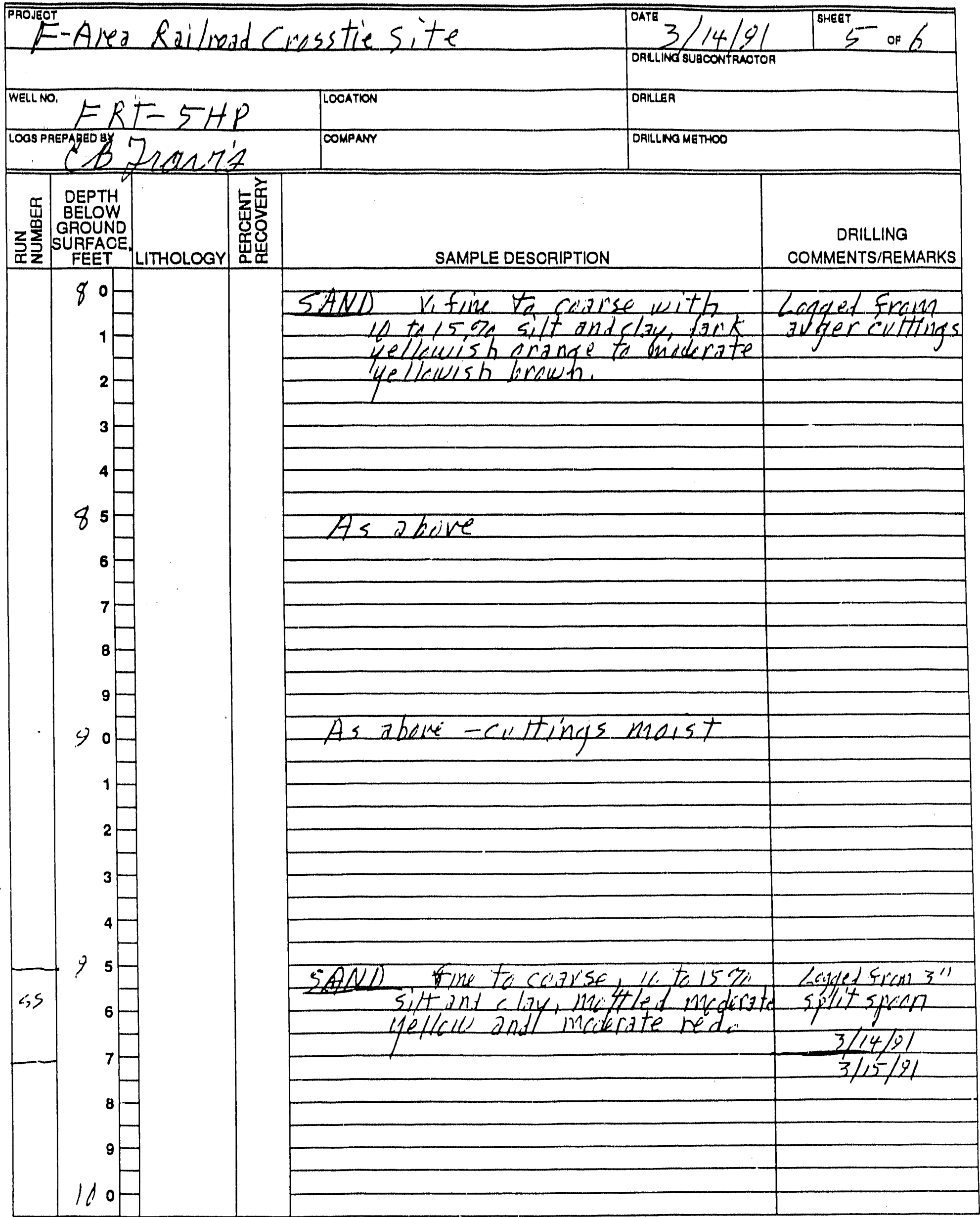




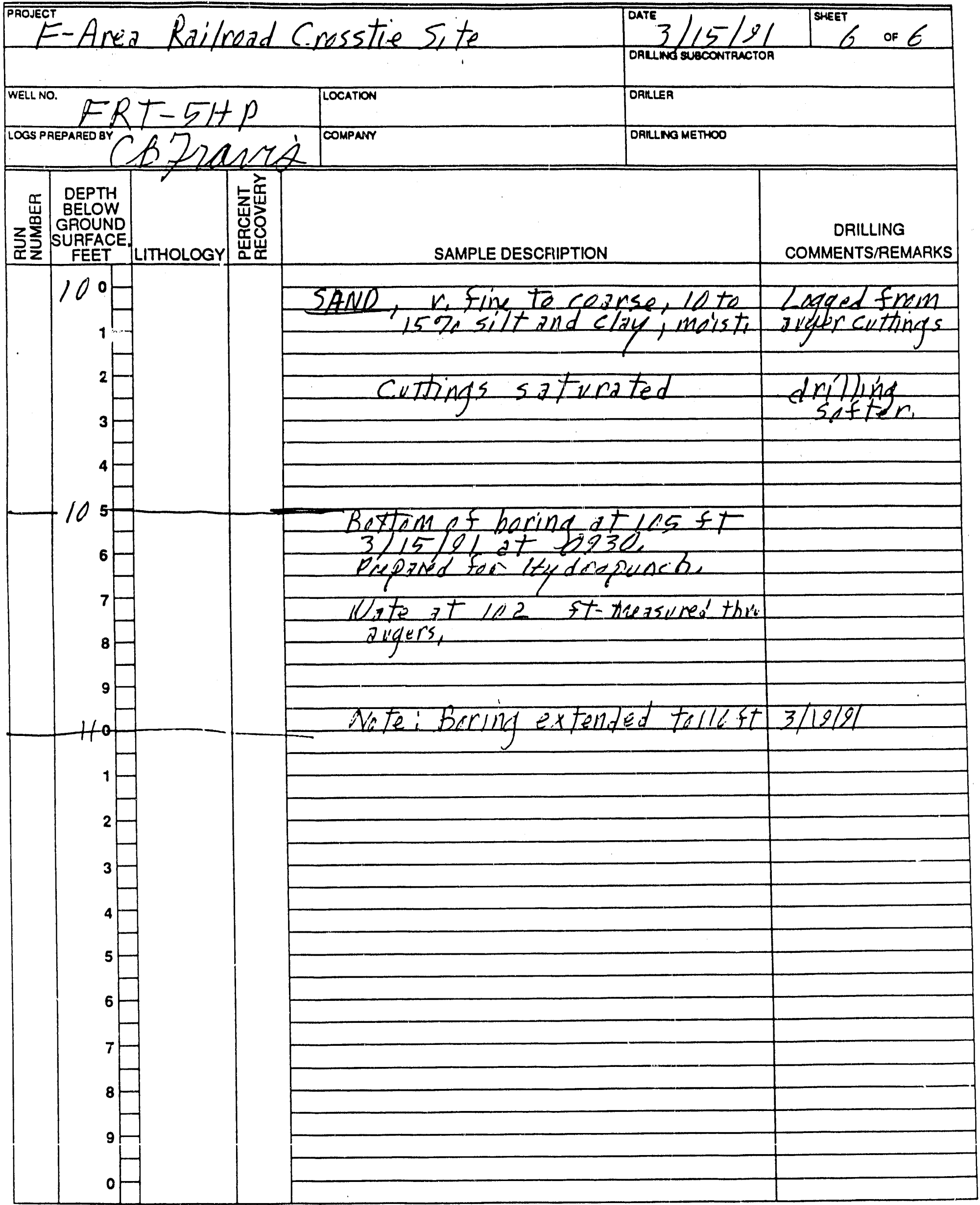




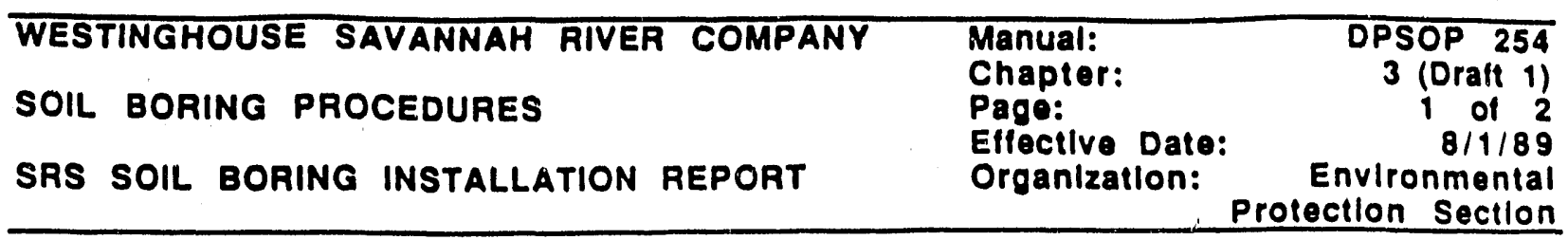

\section{SRS SOIL BORING INSTALLATION REPORT}

\section{GENERALINFORMATION}

Boring number FRT-4HI) site E-Anez Pailread Cusstielile Boring purpose Sail Baring with wiater Table sample

Project name Preliwilary characterization of the F-Ahea Raibrad Crosstie pile, Custodian I Bitter Custodial Dept $C S L^{\prime} E$

SRS Coordinates: North $8(, 151,91$ East $54,4 / 5,81$

Or: Latitude

Longitude

Location description drainage suiale adjacent to crossties

Drilling contractor Eiaves Driller Jalus Swith, \$S6I

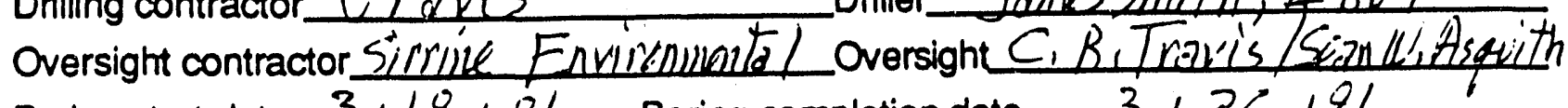
Boring start date $3119+91$ Boring completion date 3126191

\section{DRILLING INFORMATION}

Note: Depths are measured from ground surface to the nearest $0.1 \mathrm{ft}$.

Type of drill rig used

Depth to static water level

Total depth of boring

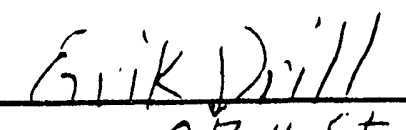

Ground surface elevation (mean sea level) $2 \$ 3,6$

Hole

Drilling Methed Diameter Erom (ft) Auger

Hollowstem auger

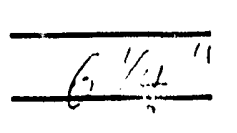

Mud rotary

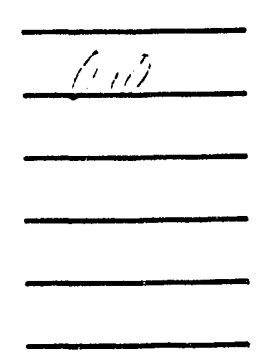

Io (tit)

Footage
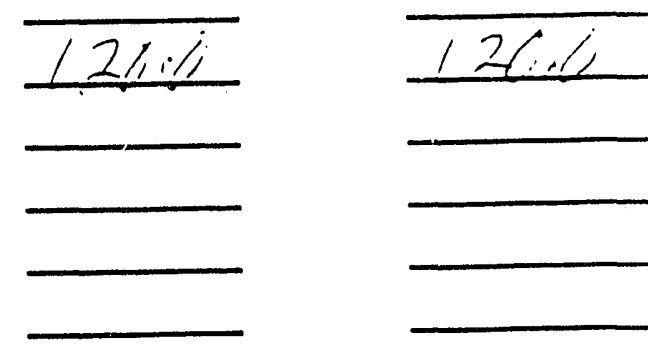

Sample depths 


\begin{tabular}{llr}
\hline WESTINGHOUSE SAVANNAH RIVER COMPANY & Manual: & OPSOP 254 \\
SOIL BORING PROCEDURES & $\begin{array}{l}\text { Chapter: } \\
\text { Page: }\end{array}$ & $\begin{array}{l}\text { Eraft } 1) \\
\text { of } 2\end{array}$ \\
SRS SOIL BORING INSTALLATION REPORT & $\begin{array}{l}\text { Organlizatlon: } \\
8 / 1 / 89\end{array}$ \\
& & $\begin{array}{r}\text { Environmental } \\
\text { Protectlon Secllon }\end{array}$ \\
\hline
\end{tabular}

Split spoon:

Sample depths $(1,1,4,1,5$ to $2,5,3,3$ t. $4,0,4.5$ t.6.l, 11 e te 12,5 It e te $17 \%, 26$ if t. 215

Other (spocity): winter samplezt unater table (z s s ft)

Sample depths

Disposition of samples Shyped to lestankab

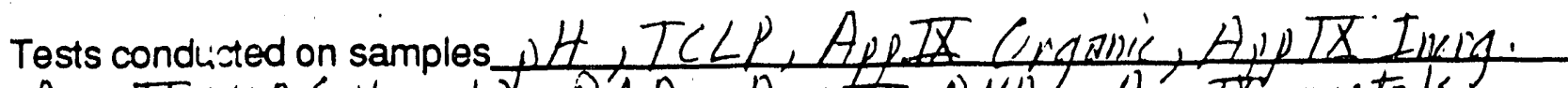

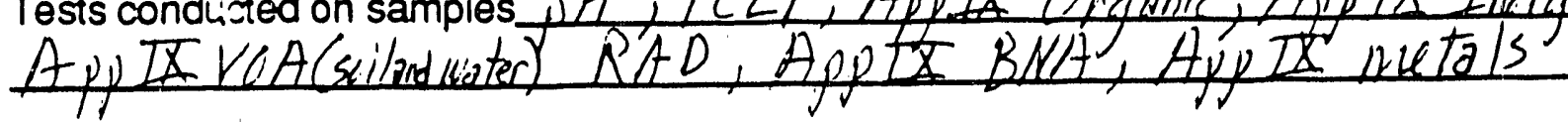

\section{BORING ABANDONMENT}

Material(s) used to abandon boring West cementgrevt with Rentante

Method of abandonment livined gruet Threight Tremié

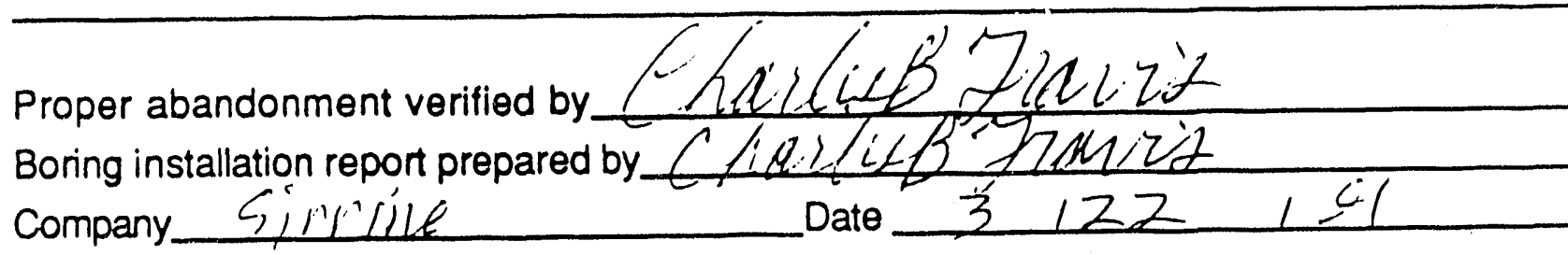

\section{COMMENTS}


FIELD GEOLOGIC LOG

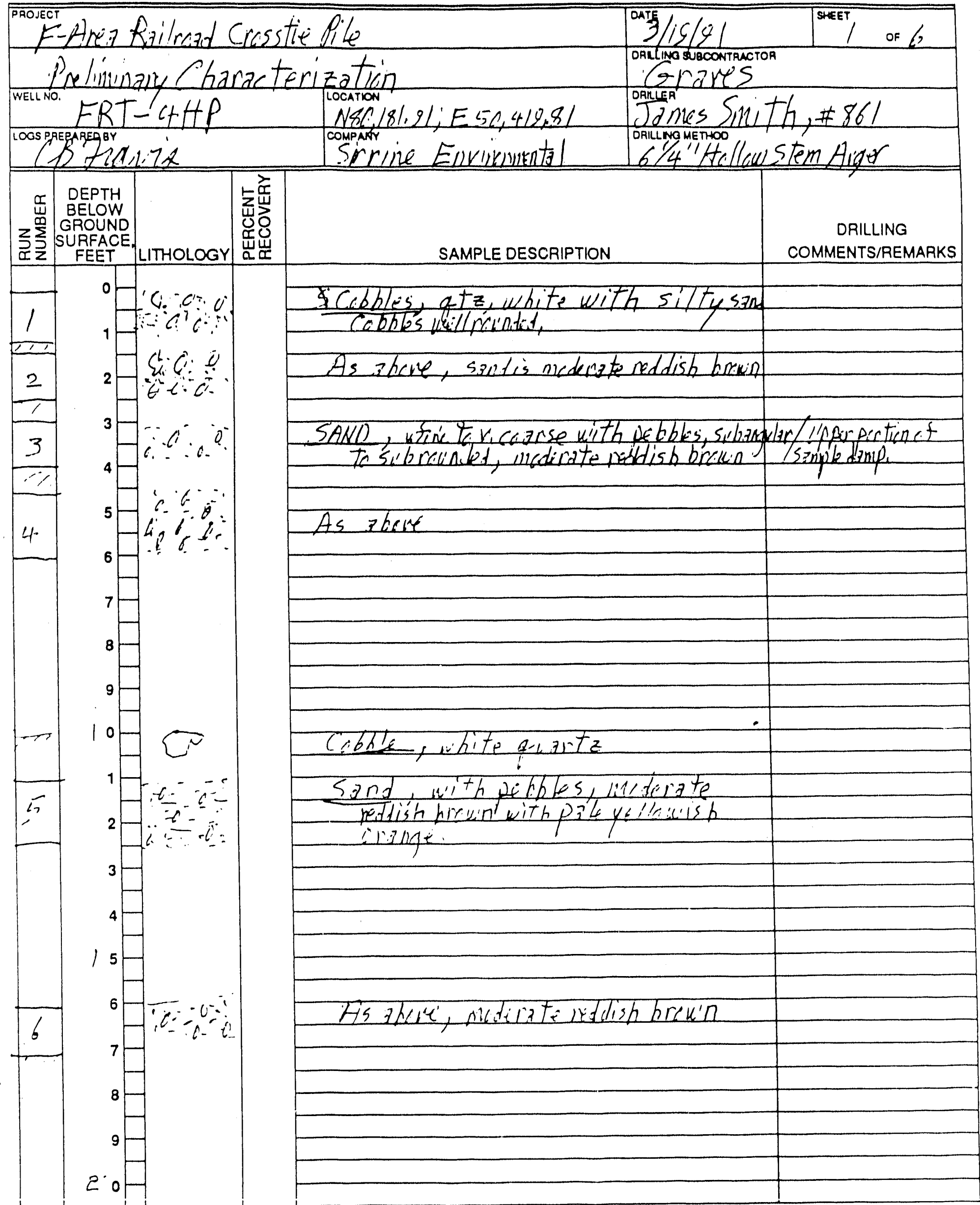




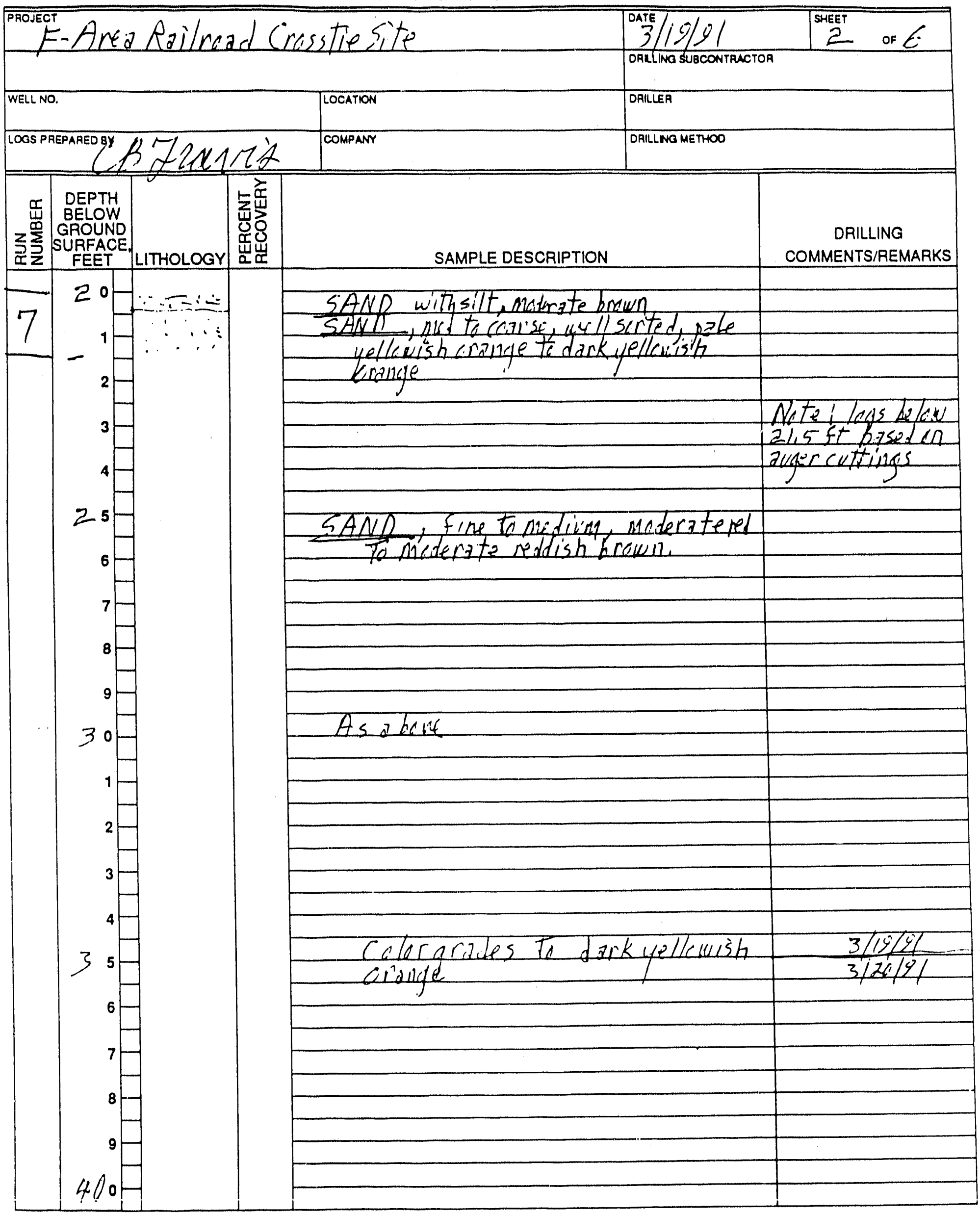




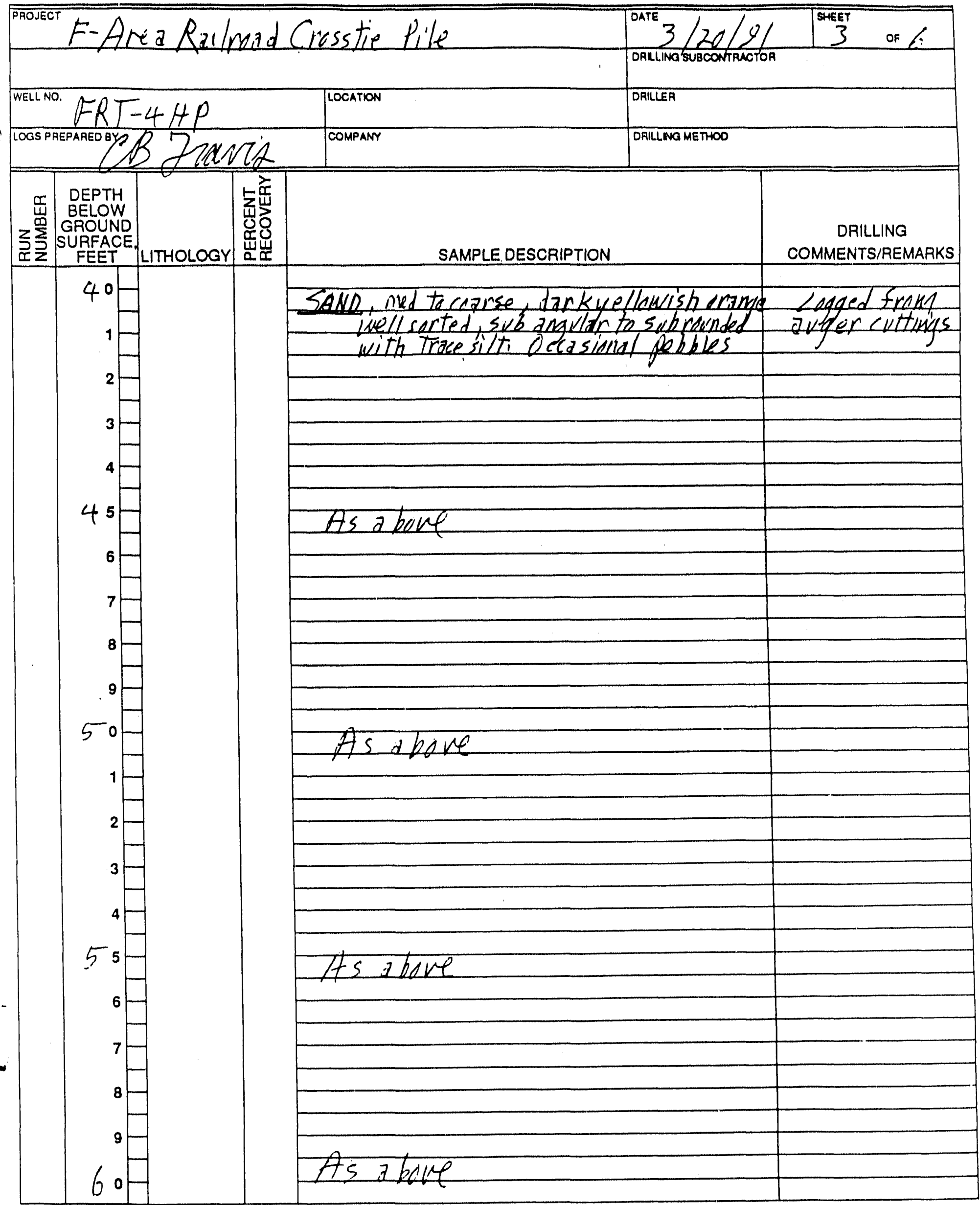


FIELD GEOLOGIC LOG

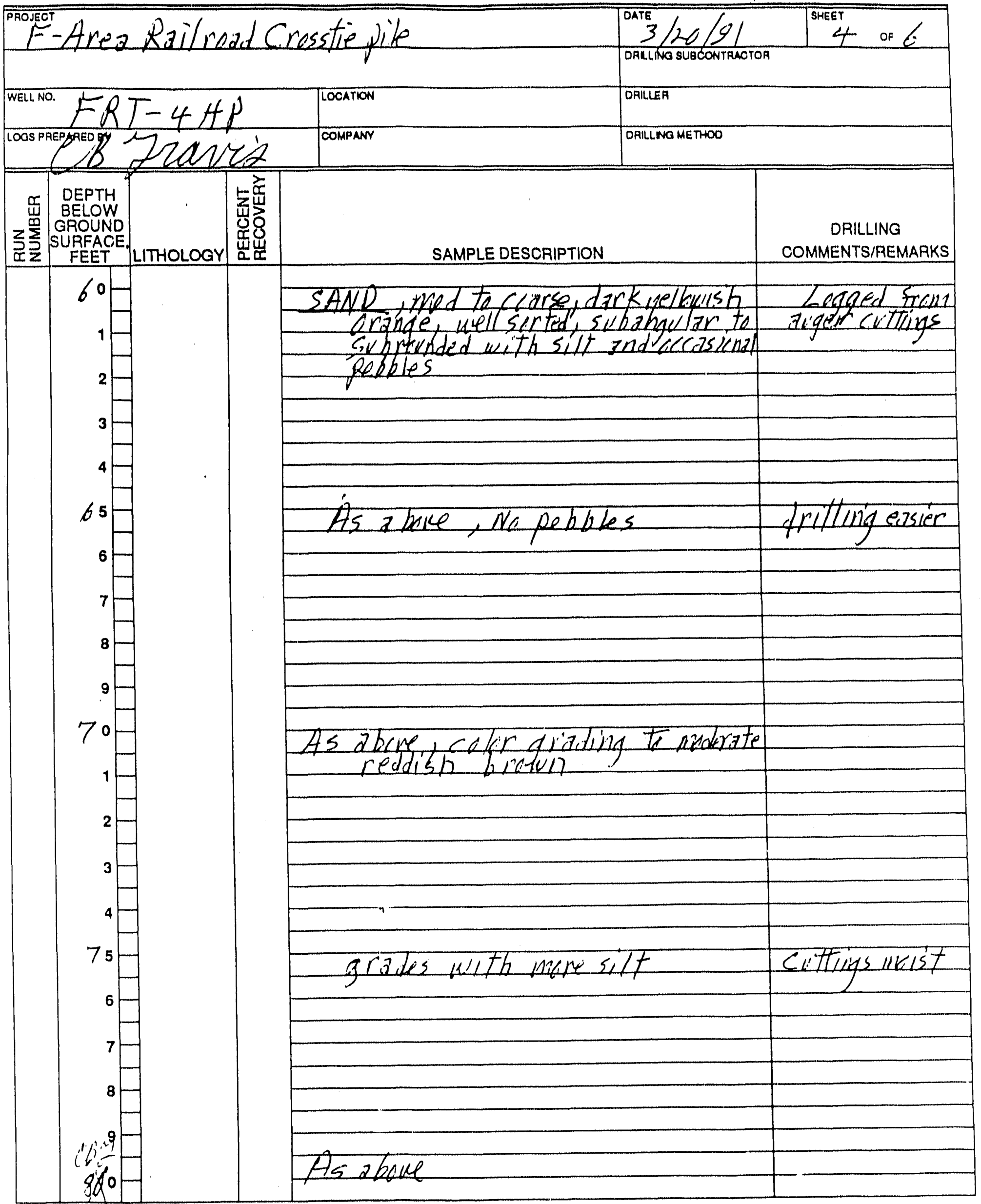


DATE

OALLING SUBCONTRACTOR

DAILLEA

ERT-4HP

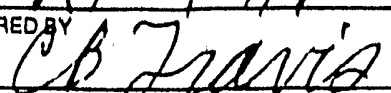

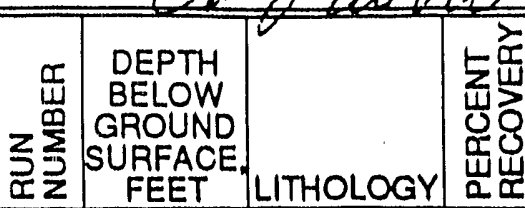

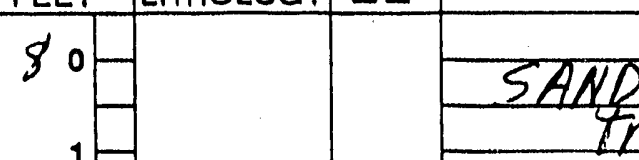

SAMPLE DESCRIPTION

DRILLING COMMENTS/REMARKS

med to coarse with siltand race clace, subangular to sibreanded maderate neddish brawn

As abere

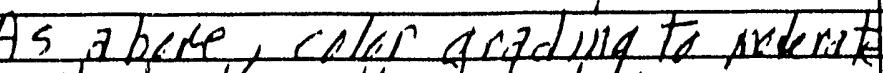
to dark yellewesh bewan.

As above

\begin{tabular}{|c|c|}
\hline & \\
\hline & \\
\hline & \\
\hline & \\
\hline & \\
\hline$A S$ & $c_{0}+t$ \\
\hline aboue & $\begin{array}{l}\text { Clifunes damp oc } \\
\text { net saturated }\end{array}$ \\
\hline
\end{tabular}




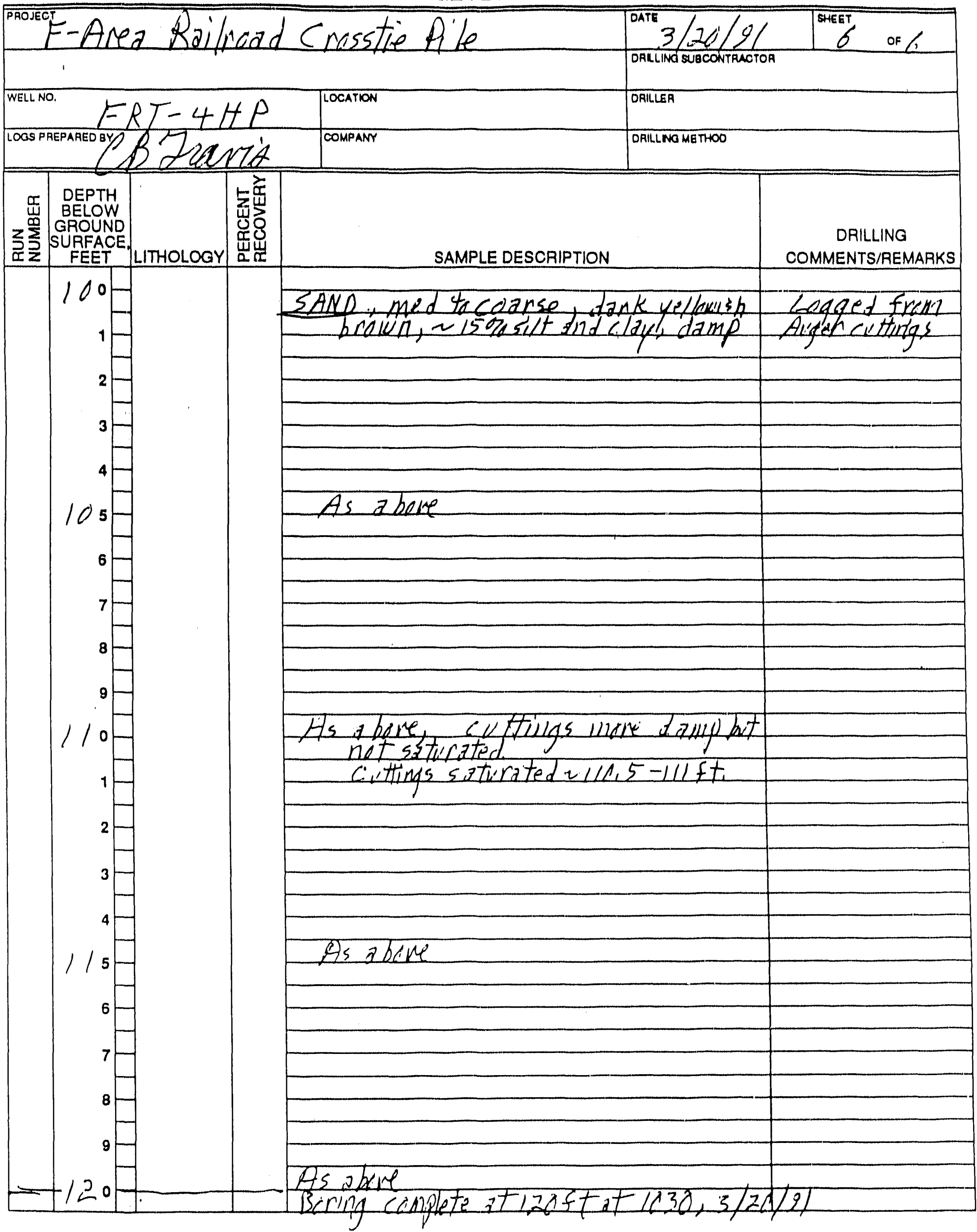




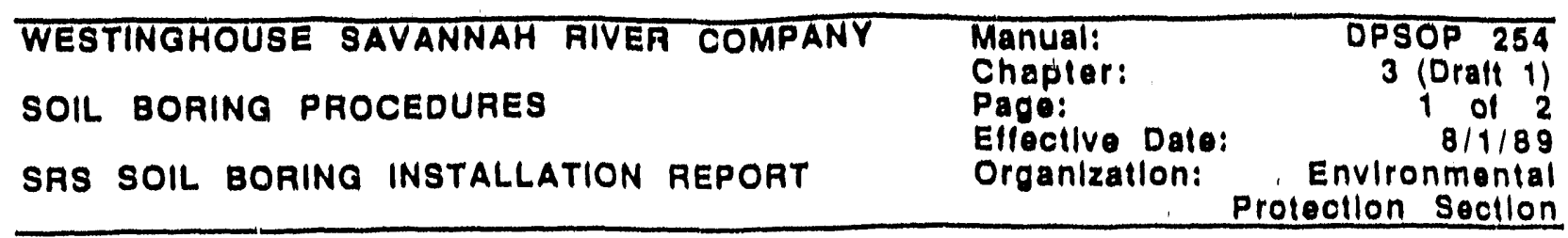

\section{SRS SOIL BORING INSTALLATION REPORT}

\section{GENERAL INEORMATION}

Boring number ERT-3HP Site E-ArezRzilrad Crusstie pile Boring purpose Siil buing 4 water toble sample Project name Preliminary Characterization of the E-Aned Railivad Cisstefle Custodian I Bittlect

SRS Coordinates: North 7\%,872,19 Custodlal Dept CSWE

Or: Latitude Longitude

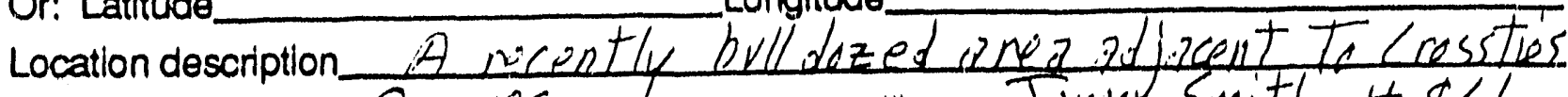
Drilling contractor arares East $E 50,39723$ Boring start date 3121121 Boring completion dato 3121121

\section{DRILLING INFORMATION}

Note: Depths are measured from ground surface to the nearest $0.1 \mathrm{ft}$.

Type of drill rig used inik inill

Depth to static water level

Total depth of boring $339 \mathrm{ft}$

Ground surface elevation (mean sea level) $2,56,3$

Hole

Drilling Methed Diameter Erom (tt) Auger

Hollowstem auger
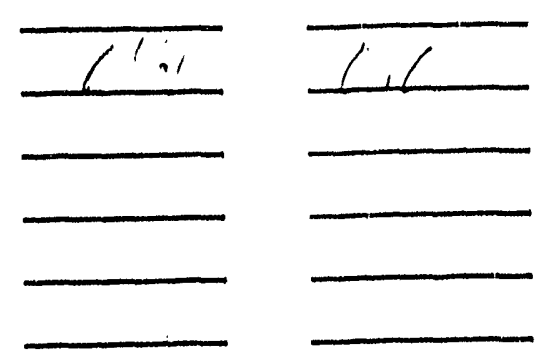

To (th) Footage Mud rotary

Sample depths 


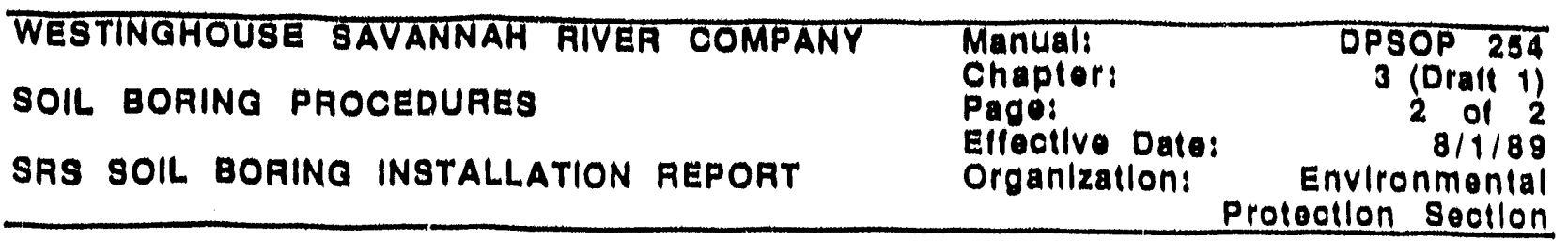

Spllt spoon:

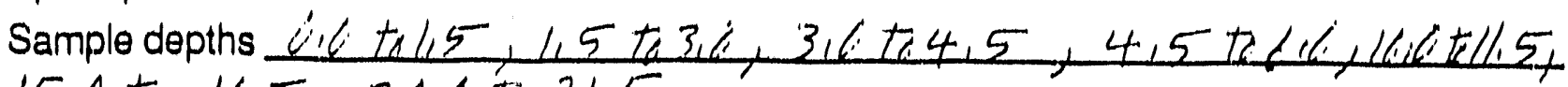
15,1 to $16,5,2010$ te 215

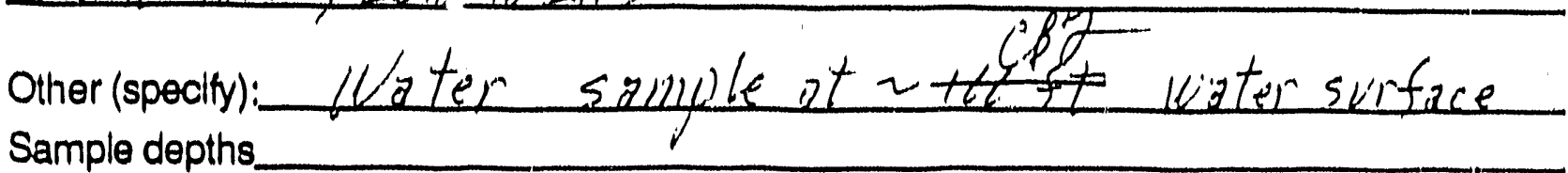

Disposition of samples Shipfed te llestell lab

Tests conducted on samples HH, TCLP, App IX Cind. An IX Iucing

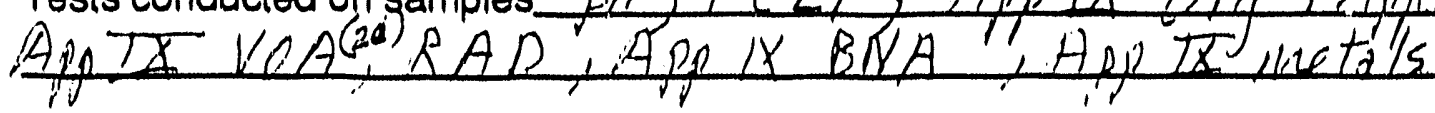

\section{BORING ABANDONMENT}

Material(s) used to abandon boring. Neat ceiluent grevt with buitewte

Method of abandonment Pimp Threvgh Trimise

Proper abandonment verifled by Boring installation report prepared by Company Siritise

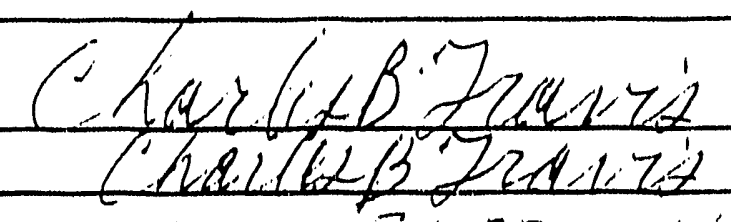
Date 3122

\section{COMMENTS}




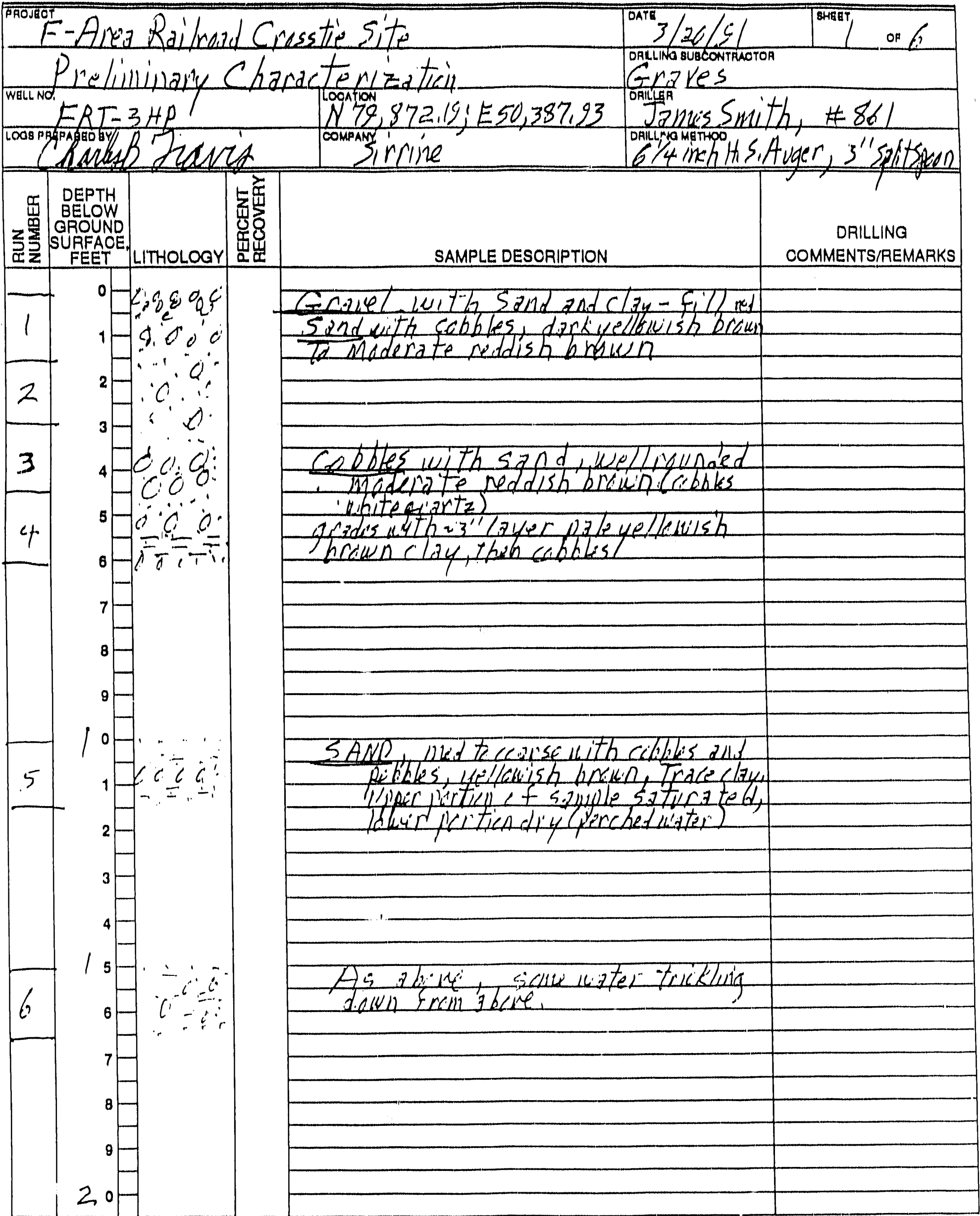




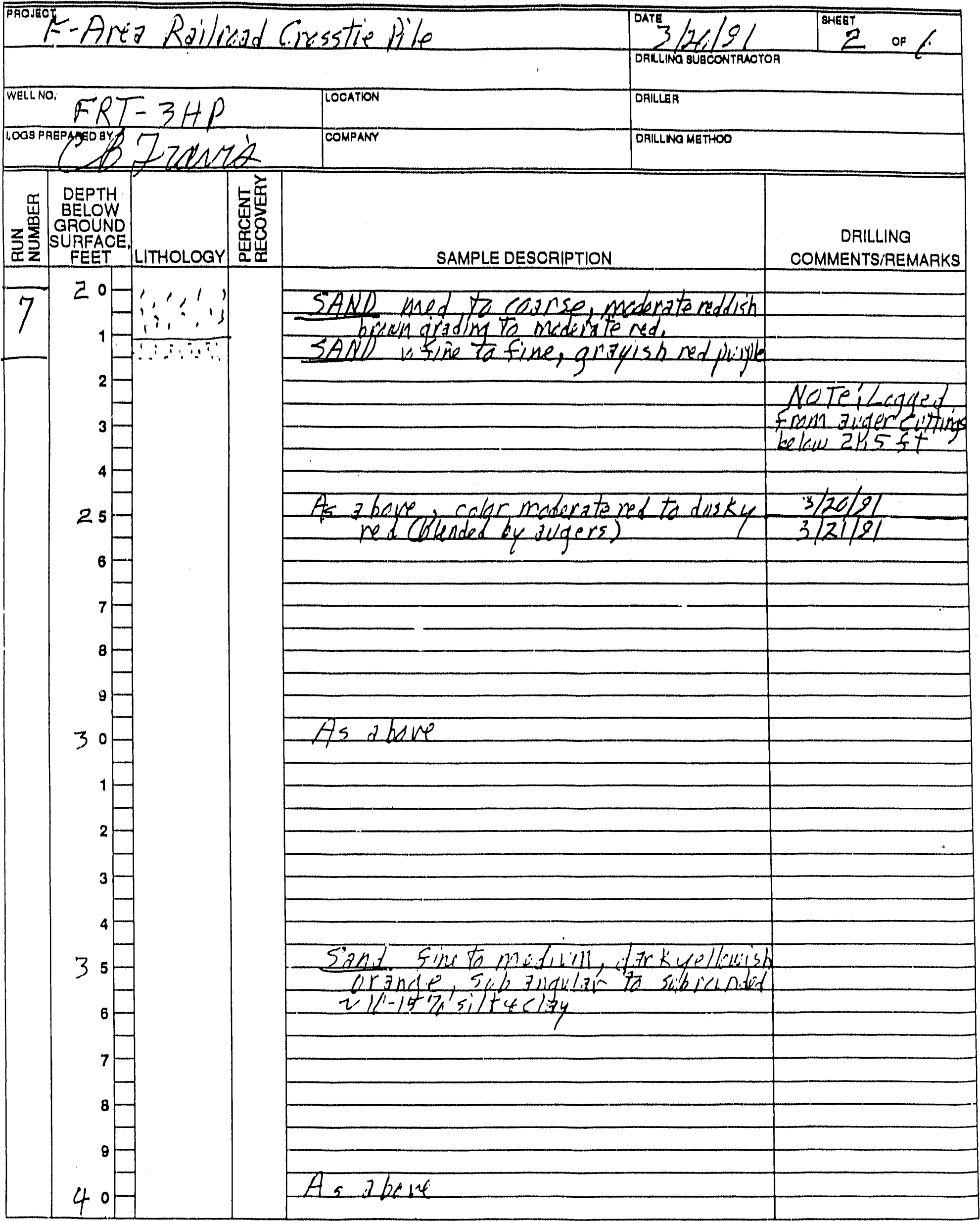




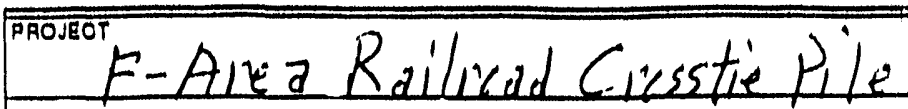

WELLNO. $F R T-3 H P$

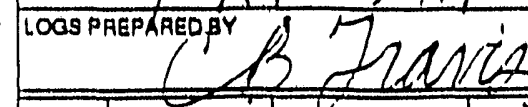
OAHLEA

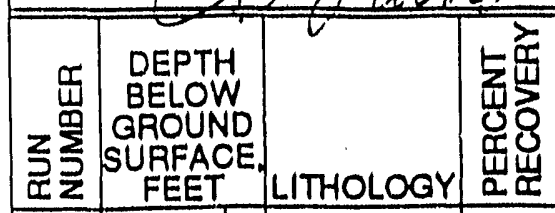

DRILLING METHOO

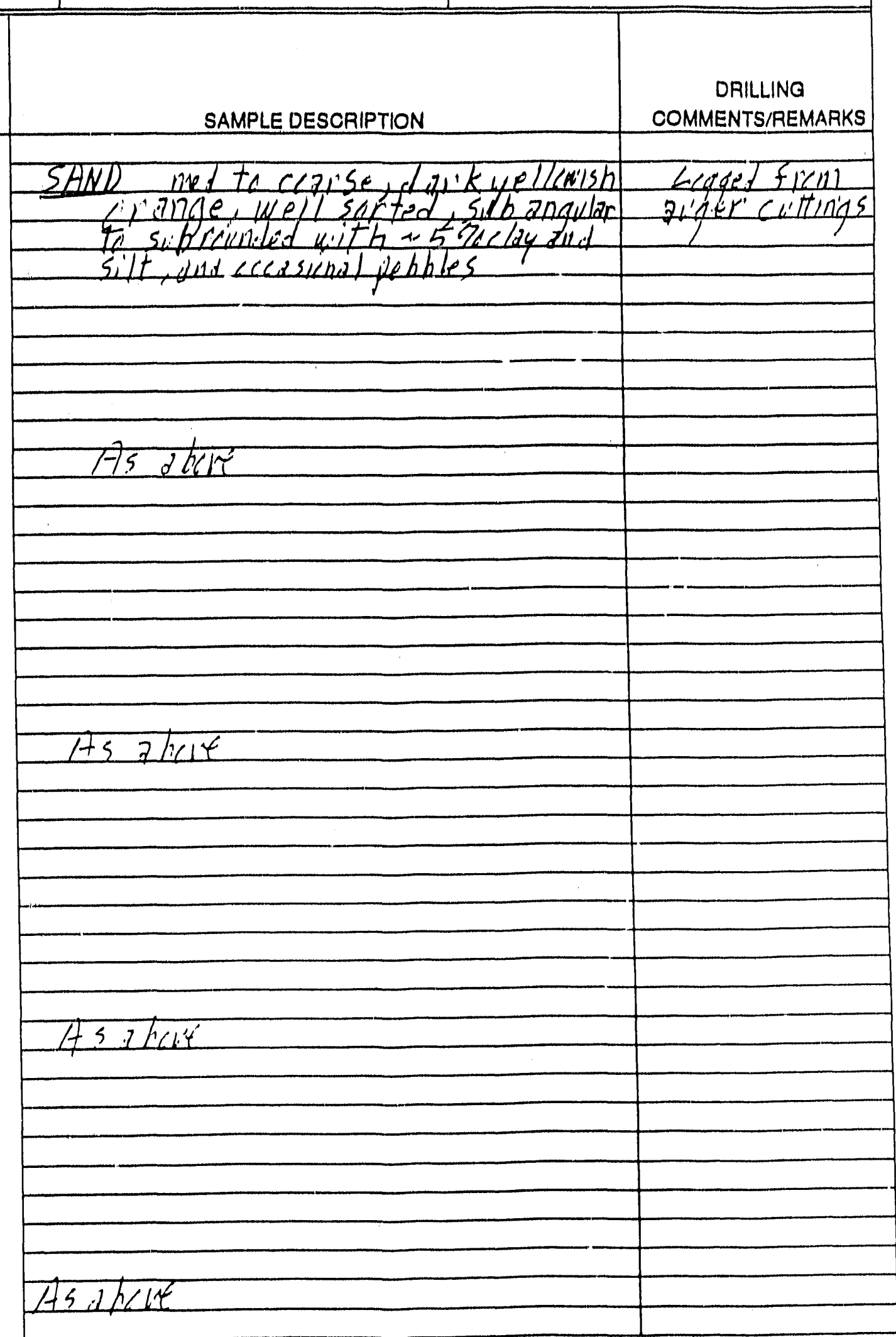




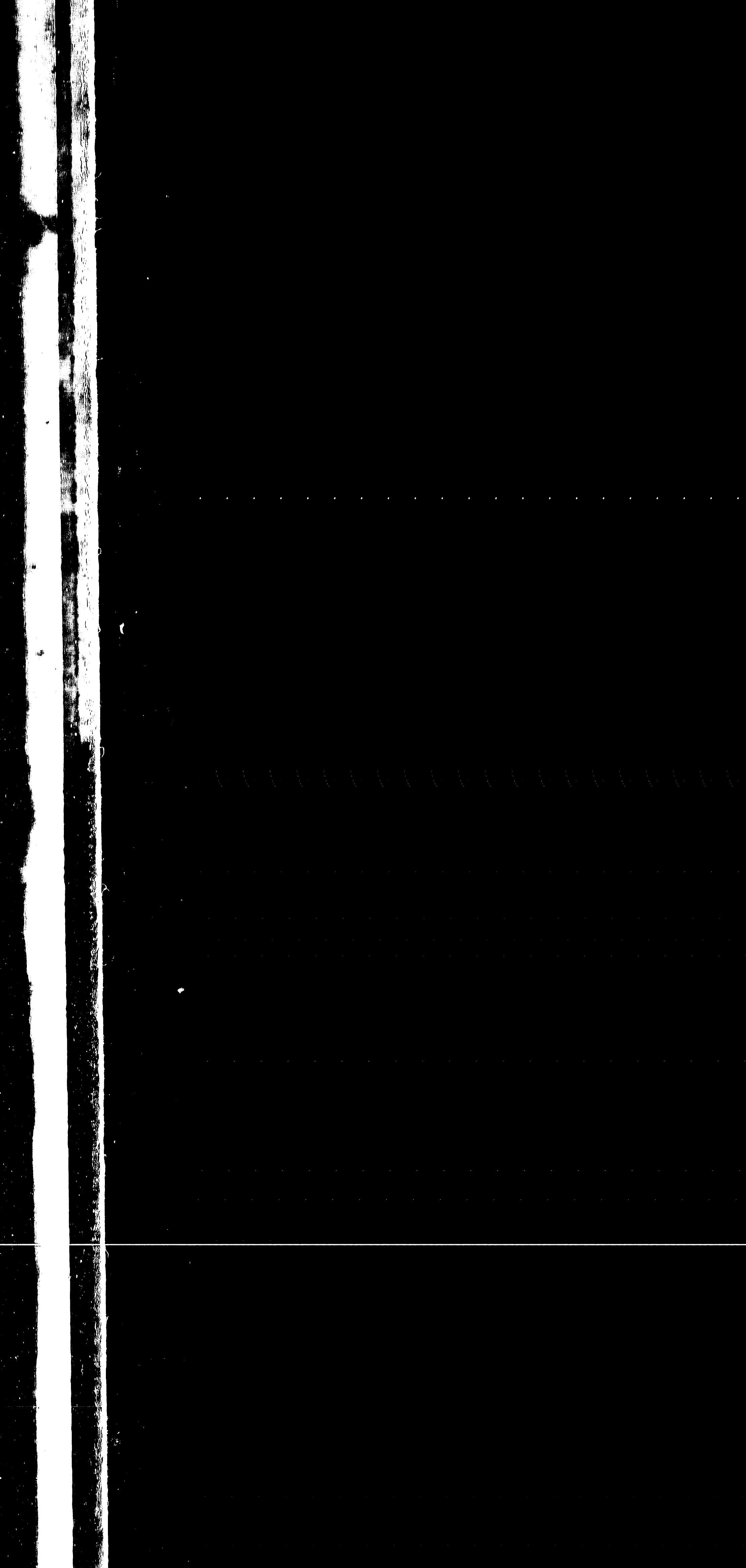


FIELD GEOLOGIC LOG

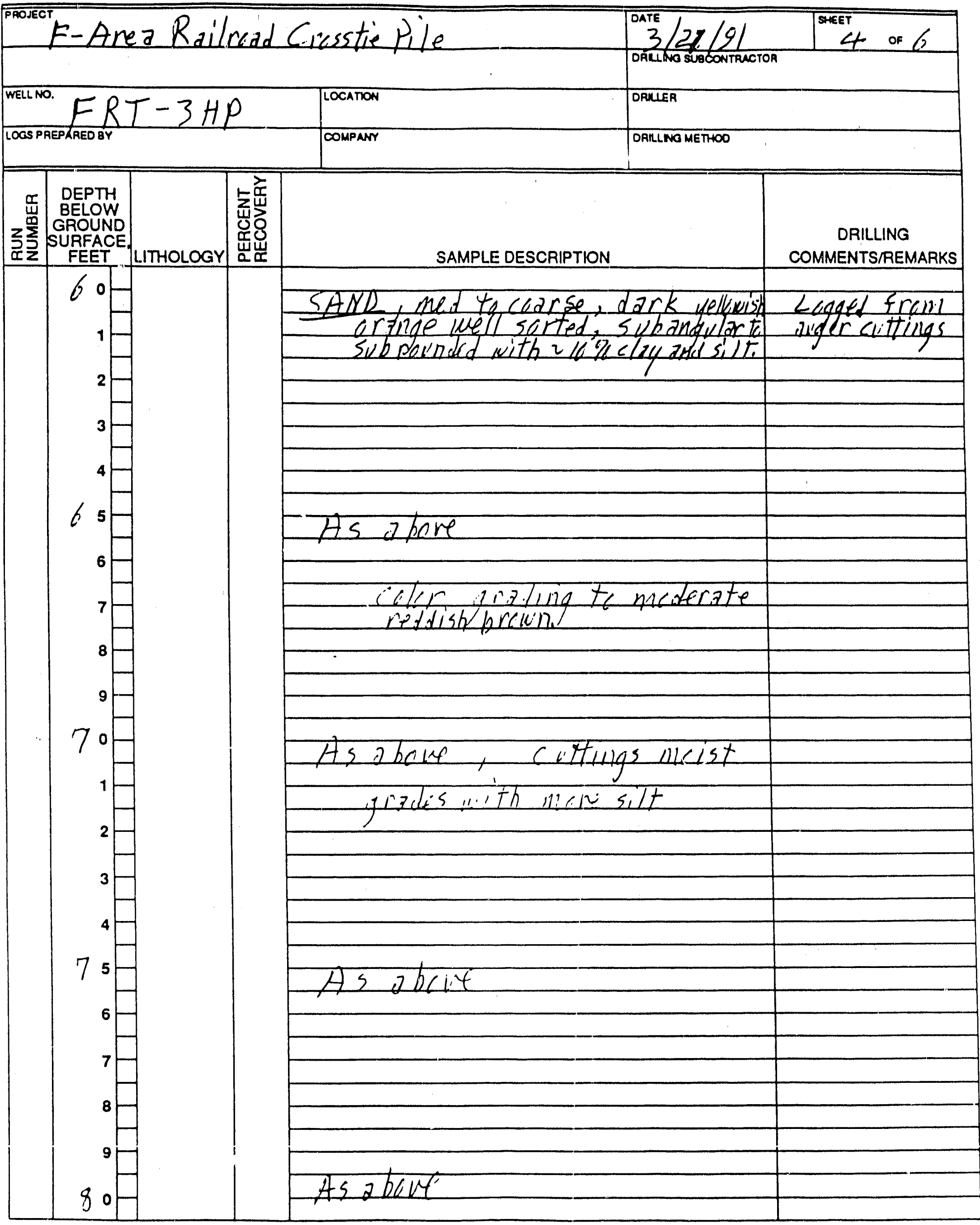




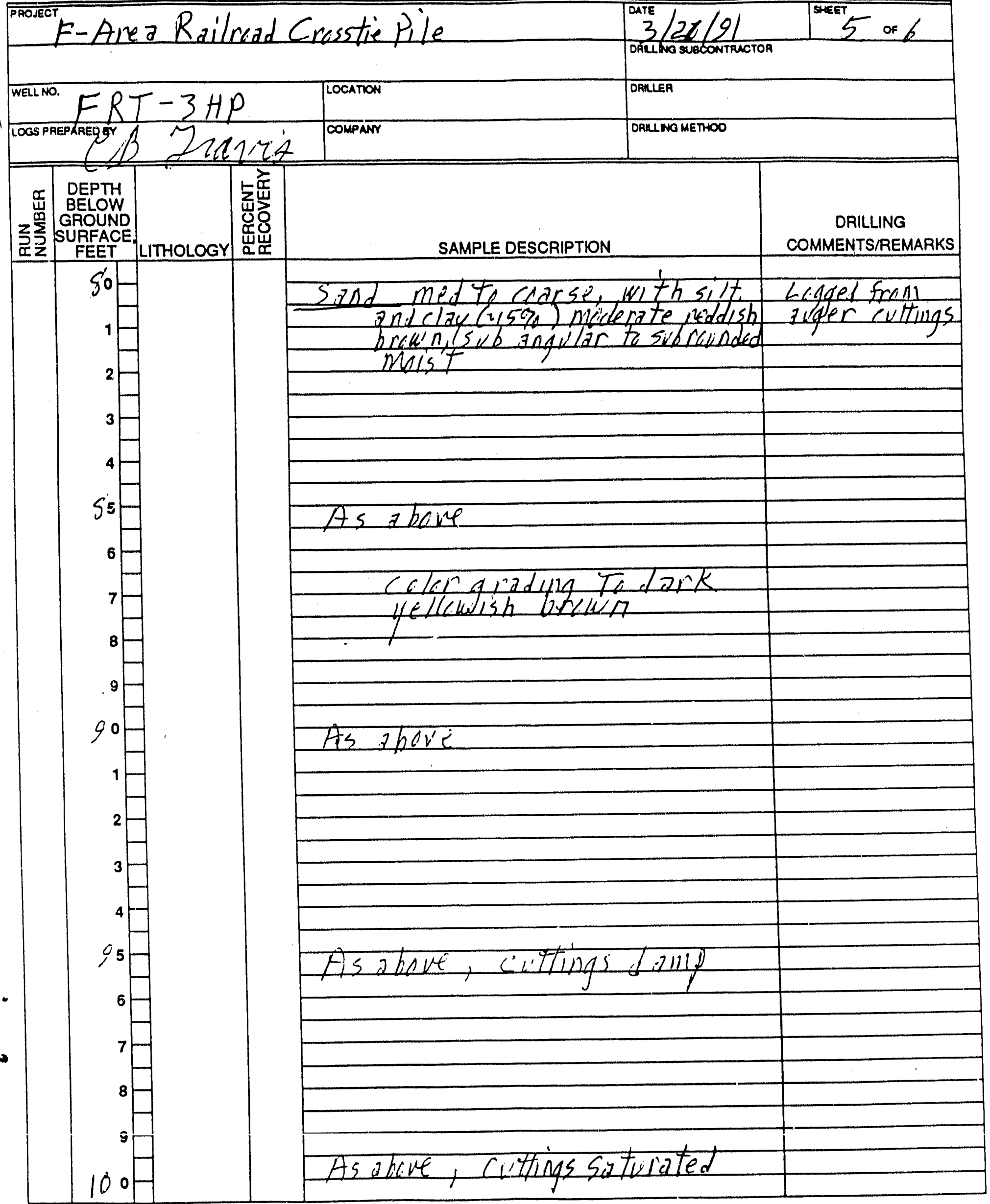


FIELD GEOLOGIC LOG

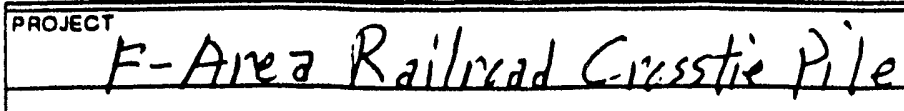

WELLO FRT-3HP

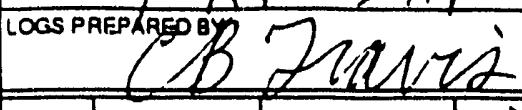

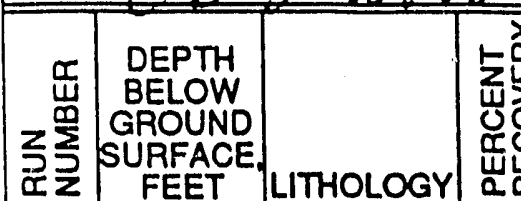
$100 \mathrm{H}$ 1 (2)

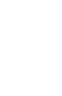

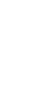
4 105 6 1 11

9 0 1 2

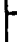

8

9 0
SAMPLE DESCRIPTION

DRILLING COMMENTS/REMARKS

SAND med to coarse dark wellewsh buen, lite $15 \%$ silt and clue, net

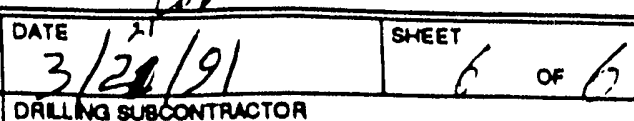

DAILEA

DFILLNG METTHOO 

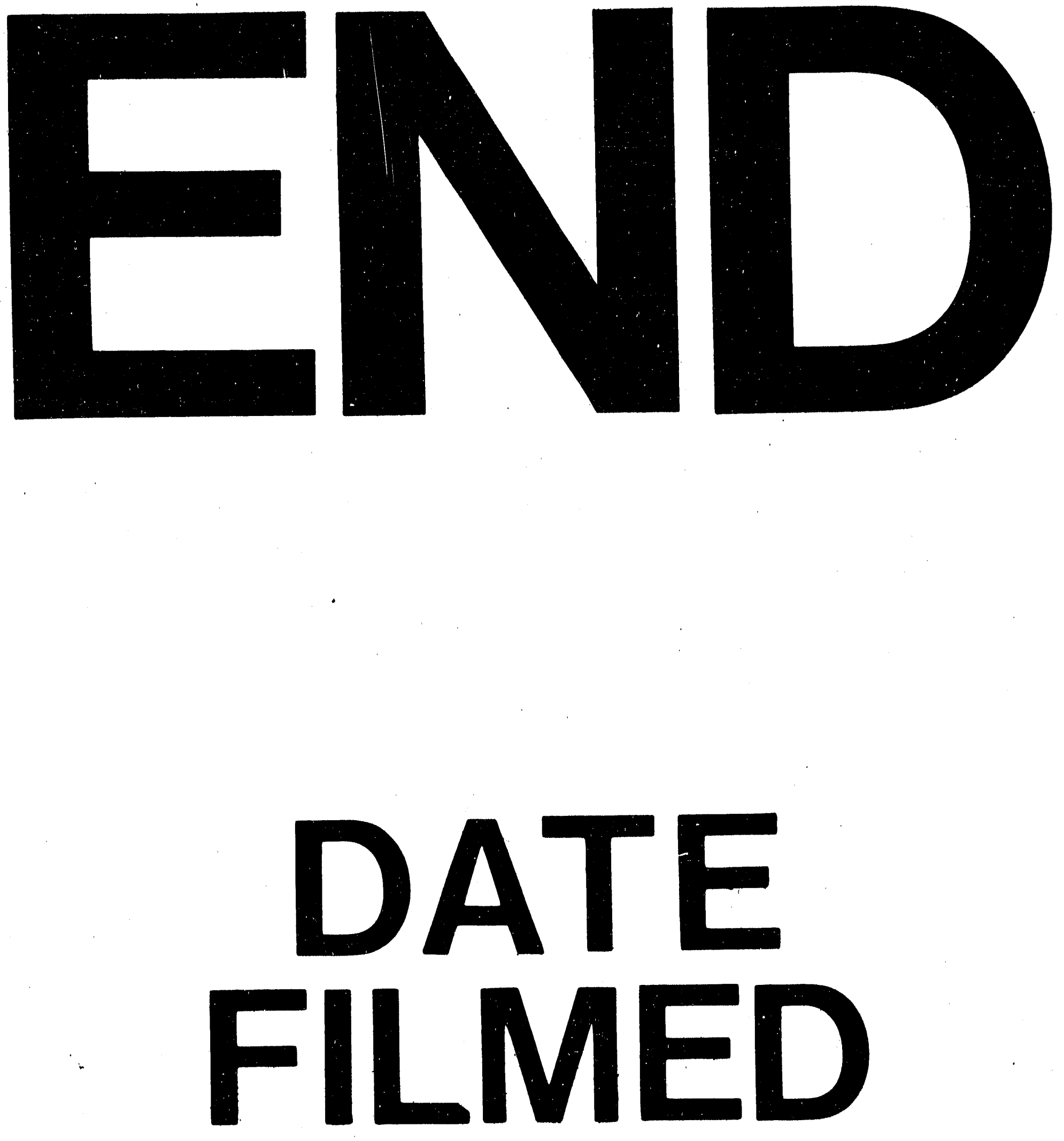

1

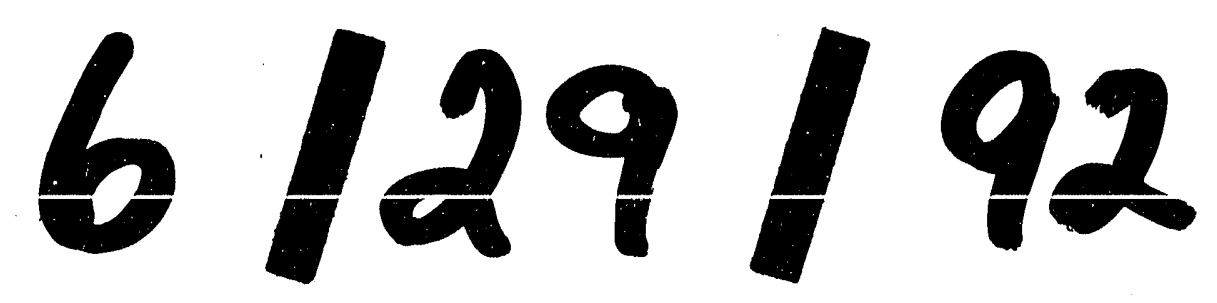


